\title{
SOME CELLULAR SUBDIVISIONS OF SIMPLICIAL COMPLEXES
}

\author{
SiBE MARDEŠIĆ \\ University of Zagreb, Croatia
}

\begin{abstract}
In a previous paper the author has associated with every inverse system of compact CW-complexes $\boldsymbol{X}$ with limit $X$ and every simplicial complex $K$ with geometric realization $|K|$ a resolution of $X \times|K|$, which consists of spaces having the homotopy type of polyhedra. In a subsequent paper it is shown that this construction is functorial. The proof depends essentially on particular cellular subdivisions of $K$. The purpose of this paper is to describe in detail these subdivisions and establish their relevant properties. In particular, one defines two subdivisions $L(K)$ and $N(K)$ of $K$. Each cell from $L(K)$, respectively from $N(K)$, is contained in a simplex $\sigma \in K$ and it is the direct sum $a \oplus b$, respectively $c \oplus d$, of certain simplices contained in $\sigma$. One defines new subdivisions $L^{\prime}(K)$ and $N^{\prime}(K)$ of $K$ by taking for their cells the direct sums $L(a) \oplus b$, respectively $c \oplus N(d)$. The main result asserts that there is an isomorphism of cellular complexes $\vartheta: L^{\prime}(K) \rightarrow N^{\prime}(K)$, which induces a selfhomeomorphism $\theta:|K| \rightarrow|K|$.
\end{abstract}

\section{INTRODUCTION}

In [6] the author has associated with every inverse system of compact Hausdorff spaces $\boldsymbol{X}=\left(X_{\lambda}, p_{\lambda \lambda^{\prime}}, \Lambda\right)$ with limit $\boldsymbol{p}=\left(p_{\lambda}, \Lambda\right): X \rightarrow \boldsymbol{X}$ and every simplicial complex $K$ with geometric realization $P=|K|$ a resolution (inverse limit with additional properties) $\boldsymbol{q}=\left(q_{\mu}, M\right): Y \rightarrow \boldsymbol{Y}=\left(Y_{\mu}, q_{\mu \mu^{\prime}}, M\right)$ of the Cartesian product $Y=X \times P$, here called the standard resolution of $X \times P$. If $\boldsymbol{X}$ consists of compact CW-complexes $X_{\lambda}$, the inverse system $\boldsymbol{Y}$ consists of spaces $Y_{\mu}$ having the homotopy type of polyhedra. In [7], it is shown that the construction of the standard resolution of $X \times P$ can be enriched so as to obtain a functor (in the first variable) from the coherent homotopy category

2010 Mathematics Subject Classification. 55U10, 52B11, 54C56.

Key words and phrases. Convex polytope, simplicial complex, cellular complex, subdivision of a complex, isomorphism of cellular complexes, resolution of a space, shape. 
of inverse systems of Hausdorff compacta $\mathrm{CH}$ (pro-Cpt) to the pro-category pro-HTop of the homotopy category HTop (for these notions and the notion of resolution see [5]). The proof depends essentially on constructing particular cellular subdivisions of $K$. The purpose of this paper is to describe in detail these subdivisions and establish their properties needed in [7].

The first construction, described in the present paper, associates with every simplicial complex $K$ in a real vector space $V$ a particular cellular subdivision $L(K)$ to which we refer as to the first canonical subdivision of $K$ (see Theorem 3.6). It is the union of subdivisions $L(\sigma)$, where $\sigma \in K$. An important property of $L(\sigma)$ is that, for an $n$-dimensional simplex $\sigma \in K$, the $n$-dimensional cells $c \in L(\sigma)$, are direct sums of the form $c=e \oplus d$, where $(e, d)$ is an ordered pair of simplices contained in $\sigma$, and the second summand $d$ is endowed with a natural linear ordering of its vertices, i.e., it is an ordered simplex. The direct sum $e \oplus d$ is a cell, homeomorphic to the Cartesian product $e \times d$, described in Section 3 .

In [7] the subdivision $L(K)$ is used in defining the desired functor $\mathrm{CH}$ (pro$\mathrm{Cpt}) \rightarrow$ pro-HTop. More precisely, with every coherent mapping $\boldsymbol{f}: \boldsymbol{X} \rightarrow \boldsymbol{X}^{\prime}$ between cofinite inverse systems $\boldsymbol{X}$ and $\boldsymbol{X}^{\prime}$, which are objects of $\mathrm{CH}$ (proCpt), one associates an induced homotopy mapping $\boldsymbol{g}=\left(g, g_{\mu}\right): \boldsymbol{Y} \rightarrow \boldsymbol{Y}^{\prime}$ between the standard resolutions $\boldsymbol{Y}$ of $X \times P$ and $\boldsymbol{Y}^{\prime}$ of $X^{\prime} \times P$. Here $g: M^{\prime} \rightarrow M$ is an increasing function, which to every $\mu \in M^{\prime}$ assigns an increasing function $g(\mu): K \rightarrow \Lambda$. Moreover, each $g_{\mu}$ is a mapping $Y_{g(\mu)} \rightarrow Y_{\mu}^{\prime}$, induced by mappings $\tilde{g}_{\mu}^{\sigma}: X_{(g(\mu))(\sigma)} \times \sigma \rightarrow Y_{\mu}^{\prime}$, where $\sigma$ ranges over $K$. The latter mappings are given by explicit formulae on subsets $X_{(g(\mu))(\sigma)} \times c$, where $c$ ranges over the set of cells of $L(\sigma)$.

A particular cellular subdivision $N\left(\Delta^{n}\right)$ of the standard $n$-simplex $\Delta^{n}$ was used in defining the composition $\boldsymbol{f}^{\prime \prime}=\boldsymbol{f}^{\prime} \boldsymbol{f}: \boldsymbol{X} \rightarrow \boldsymbol{X}^{\prime \prime}$ of two coherent mappings $\boldsymbol{f}: \boldsymbol{X} \rightarrow \boldsymbol{X}^{\prime}$ and $\boldsymbol{f}^{\prime}: \boldsymbol{X}^{\prime} \rightarrow \boldsymbol{X}^{\prime \prime}$ (see [5, I.1.3] or Section 4 of [7]). This subdivision consists of $n+1 n$-dimensional convex polytopes $P_{i}^{n} \subseteq \Delta^{n}$, $0 \leq i \leq n$, and of their faces. Each of these $n$-cells is the direct sum $P_{i}^{n}=$ $Q_{i}^{i} \oplus R_{i}^{n-i}$ of two simplices $Q_{i}^{i}$ and $R_{i}^{n-i}$ of dimensions $i$ and $n-i$, respectively (see Section 4 of [7]). By definition, $\boldsymbol{f}^{\prime \prime}$ consists of an increasing function $f^{\prime \prime}: \Lambda^{\prime \prime} \rightarrow \Lambda$ between the index sets of $\boldsymbol{X}^{\prime \prime}$ and $\boldsymbol{X}$ and of some mappings $f_{\boldsymbol{\mu}}^{\prime \prime}: X_{f^{\prime \prime}\left(\mu_{n}\right)} \times \Delta^{n} \rightarrow X_{\mu_{0}}^{\prime \prime}$, where $\boldsymbol{\mu}=\left(\mu_{0}, \ldots, \mu_{n}\right)$ is a multiindex in $M^{\prime \prime}$ of length $n$. These mappings are defined by explicit formulae on the subsets $X_{f^{\prime \prime}\left(\mu_{n}\right)} \times P_{i}^{n} \subseteq X_{f^{\prime \prime}\left(\mu_{n}\right)} \times \Delta^{n}$.

The construction of the subdivision $N\left(\Delta^{n}\right)$ of $\Delta^{n}$ readily generalizes to the construction of a subdivision $N(\sigma)$ of any linearly ordered simplex $\sigma=$ $\left[v_{0}, \ldots, v_{n}\right] \subseteq V$. We refer to it as to the second canonical subdivision of $\sigma$. By definition, $N(\sigma)$ consists of $n$-dimensional polytopes $c_{i}^{\sigma}, 0 \leq i \leq n$, and of their faces. As in the case of $L(\sigma)$, each of the $n$-cells $c_{i}^{\sigma}$ is the direct sum $c_{i}^{\sigma}={c^{\prime}}_{i}^{\sigma} \oplus{c^{\prime \prime}}_{i}{ }_{i}^{\sigma}$ of two simplices ${c^{\prime}}_{i}^{\sigma}$ and ${c^{\prime \prime}}_{i}{ }_{i}$ (of dimensions $i$ and $n-i$, respectively) (see Theorem 4.3). The construction easily extends further to a 
cellular subdivision $N(K)$, for any ordered simplicial complex $K$ in $V$. Recall that $K$ is ordered if its set of vertices is partially ordered in such a way that the vertices of each of its simplices are linearly ordered.

We will consider two more cellular subdivisions of $K$ to which we refer as to the first iterated canonical subdivision $L^{\prime}(K)$ of $K$ and the second iterated canonical subdivision $N^{\prime}(K)$ of $K$. To define $L^{\prime}(K)$ we first define a cellular subdivision $L^{\prime}(\sigma)$ of $L(\sigma)$, for each $\sigma \in K$. If $\operatorname{dim} \sigma=n$, one considers the subdivision $L(\sigma)$ and its $n$-cells $c \in L(\sigma)$. They are of the form $c=e \oplus d$, where $e$ and $d$ are simplices contained in $\sigma$ of dimensions $\operatorname{dim} e=i$ and $\operatorname{dim} d=n-i$, $0 \leq i \leq n$, respectively. Applying the operation $L$ to the first summand $e$, one obtains a subdivision $L(e)$ of $e$. The $i$-cells $e^{\prime} \in L(e)$ determine $n$-cells $e^{\prime} \oplus d \subseteq c$, which together with their faces form a cellular subdivision $L(c)$ of $c$. It is shown in Theorem 5.1 that the union of all $L(c)$, when $c$ ranges over the set of $n$-cells from $L(\sigma)$, is a cellular subdivision $L^{\prime}(\sigma)$ of $L(\sigma)$, hence also a cellular subdivision of $\sigma$. Finally, we put $L^{\prime}(K)=\cup_{\sigma \in K} L^{\prime}(\sigma)$.

Similarly, to define $N^{\prime}(K)$ we will first define a cellular subdivision $N^{\prime}(\sigma)$ of $L(\sigma)$, for each $\sigma \in K$. As above, if $\operatorname{dim} \sigma=n$, one considers the $n$-cells $c=e \oplus d$ from $c \in L(\sigma)$. Applying the operation $N$ to the second summand $d$, one obtains a subdivision $N(d)$ of $d$. The $(n-i)$-cells $d^{\prime} \in N(d)$ determine $n$-cells $e \oplus d^{\prime} \subseteq c$, which together with their faces form a cellular subdivision $N(c)$ of $c$. It is shown in Theorem 6.1 that the union of all $N(c)$, when $c$ ranges over the set of $n$-cells from $L(\sigma)$, is a cellular subdivision $N^{\prime}(\sigma)$ of $L(\sigma)$, hence also a cellular subdivision of $\sigma$. Finally, we put $N^{\prime}(K)=\cup_{\sigma \in K} N^{\prime}(\sigma)$.

A careful analysis of the cellular complexes $L^{\prime}(K)$ and $N^{\prime}(K)$ shows that there exists an isomorphism of cellular complexes $\vartheta: L^{\prime}(K) \rightarrow N^{\prime}(K)$ (Theorem 7.1). Moreover, there exist affine isomorphisms between the corresponding cells $c^{*} \in L^{\prime}(K)$ and $c^{\bullet}=\vartheta\left(c^{*}\right) \in N^{\prime}(K)$, which generate a selfhomeomorphism $\theta: P \rightarrow P$ of the carrier $P=|K|=\left|L^{\prime}(K)\right|=\left|N^{\prime}(K)\right|$ (Theorem 7.2). These surprising facts are the main results of this paper and have enabled the author to prove in [7] the following assertion. The composition $\boldsymbol{g}^{\prime} \boldsymbol{g}$ of the homotopy mappings $\boldsymbol{g}$ and $\boldsymbol{g}^{\prime}$, associated with $\boldsymbol{f}$ and $\boldsymbol{f}^{\prime}$, respectively, is homotopic to the homotopy mapping $\boldsymbol{g}^{\prime \prime}$, associated with the composition $\boldsymbol{f}^{\prime \prime}=\boldsymbol{f}^{\prime} \boldsymbol{f}$. This assertion is the crucial step in establishing the desired functoriality of the standard resolution of $X \times P$.

\section{Preliminaries on CONVEX POlytopes AND CEllular COMPlexes}

In this section we fix terminology and notations and review notions and facts from the basic theory of polytopes and cellular complexes, needed throughout the paper.

2.1. Let $V$ be a (real) vector space. The sum of two non-empty subsets $A, B \subseteq V$ is the set $A+B=\{a+b \mid a \in A, b \in B\} \subseteq V$. An affine manifold $M$ in $V$ is a subset of $V$ of the form $M=v+L_{M}$, where $v \in V$ and $L_{M}$ 
is a linear subspace of $V$. Note that $L_{M}$ is completely determined by $M$, i.e., it does not depend on the choice of $v$. An alternative definition says that $M$ is an affine manifold in $V$ if $v_{0}, \ldots, v_{k} \in M, \lambda_{0}, \ldots, \lambda_{k} \in \mathbb{R}$ and $\sum_{i=0}^{i=k} \lambda_{i}=1$ imply $\sum_{i=0}^{i=k} \lambda_{i} v_{i} \in M$. By definition, the (geometric) dimension $\operatorname{dim} M=\operatorname{dim} L_{M}$. A set of points $\left\{v_{0}, \ldots, v_{n}\right\} \subseteq V$ is affinely independent if its affine hull $\operatorname{Aff}\left(\left\{v_{0}, \ldots, v_{n}\right\}\right)$ has dimension $n$.

A mapping $f: M \rightarrow N$ between affine manifolds $M=v+L_{M}$ and $N=$ $w+L_{N}$ is an affine mapping provided there is a linear mapping $\varphi: L_{M} \rightarrow L_{N}$ such that $f(v+x)=w+\varphi(x)$, for $x \in L_{M}$. Alternatively, $f$ is affine if $v_{0}, \ldots, v_{k} \in M, \lambda_{0}, \ldots, \lambda_{k} \in \mathbb{R}$ and $\sum_{i=0}^{i=k} \lambda_{i}=1$ imply $f\left(\sum_{i=0}^{i=k} \lambda_{i} v_{i}\right)=$ $\sum_{i=0}^{i=k} \lambda_{i} f\left(v_{i}\right)$.

A convex polytope $C$ in $V$ is the convex hull $C=\operatorname{Conv}(F)$ of a finite set of points $F \subseteq V$. Dimension $\operatorname{dim} C$ is the dimension of the affine hull $\operatorname{Aff}(C)$ (see $[2, \S 7]$ or $\left[9\right.$, Definition 6.2.1]). A proper face $C^{\prime}$ of a convex polytope $C$ is the intersection of $C$ with a supporting hyperplane of $C$ in $\operatorname{Aff}(C)$; we use the notation $C^{\prime}<C$ (see $[4, \S 2.4]$ or $[2$, Theorem 7.5] or $[9, \S 6.2])$. The empty set $\emptyset$ and $C$ itself are improper faces of $C$. If $C^{\prime}$ is a face of $C$, proper or improper, we use the notation $C^{\prime} \leq C$. The faces of a convex polytope are convex polytopes (see [2, Theorem 7.3] or [9, Lemma 6.2.3]). If $C$ is a convex polytope in $V, C^{\prime} \leq C$ and $C^{\prime \prime} \leq C^{\prime}$, then $C^{\prime \prime} \leq C$ (see [4, §3.5]). Two faces of a convex polytope intersect in a common face (possibly empty) (see $[4,2.4 .10]$ or $[2$, Theorem 5.9]). The (geometric) boundary $\operatorname{Bd}(C)$ of $C$ is the union of all proper faces of $C$. The (geometric) interior $\operatorname{Int}(C)$ of $C$ is the set $C \backslash \mathrm{Bd}(C)$. Vertices of $C$ are the 0 -dimensional faces of $C$. Every convex polytope is the convex hull of its vertices (see [2, Theorem 7.2] or [9, Theorem 6.2.5]). The faces of a convex polytope $C$ of dimension $\operatorname{dim} C-1$ are called facets of $C$. Every proper face of $C$ is a face of some facet of $C$. The boundary $\operatorname{Bd}(C)$ equals the union of all facets of $C$.

A mapping $f: C \rightarrow C^{\prime}$ between convex polytopes is said to be affine provided it is the restriction to $C$ of an affine mapping between the affine hulls $\operatorname{Aff}(C)$ and $\operatorname{Aff}\left(C^{\prime}\right)$. In 3.7.3.1, we will need the following elementary lemma (see the proof of Lemma 3.11.(i)).

Lemma 2.1. Let $f: C \rightarrow C^{\prime}$ be an affine mapping between two convex polytopes and let $D<C$ and $D^{\prime}<C^{\prime}$ be proper faces such that $f(D) \subseteq D^{\prime}$. If for every vertex $w \in C \backslash D$ one has $f(w) \in C^{\prime} \backslash D^{\prime}$, then for every point $u \in C \backslash D$, one has $f(u) \in C^{\prime} \backslash D^{\prime}$.

Proof. Since $C$ is the convex hull of its vertices, there exist vertices $w_{0}, \ldots, w_{n}$ of $C$ and there exist real numbers $\mu_{0}, \ldots, \mu_{n} \geq 0$ such that $\sum_{0 \leq i \leq m} \mu_{i}=1$ and $u=\sum_{0 \leq i \leq m} \mu_{i} w_{i}$. At least one of the vertices $w_{i}$ does not belong to $D$ and the corresponding coefficient is $\neq 0$, for otherwise, we would have $u \in D$. Therefore, there is no loss of generality in assuming that either all vertices $w_{0}, \ldots, w_{n} \in C \backslash D$ or there is a $k, 0 \leq k<n$, such that 
$w_{0}, \ldots, w_{k} \in D, w_{k+1}, \ldots w_{n} \in C \backslash D$ and $\mu_{k+1}, \ldots \mu_{n}>0$. In the first case, by assumption, $f\left(w_{0}\right), \ldots, f\left(w_{n}\right) \in C^{\prime} \backslash D^{\prime}$. Since $C^{\prime} \backslash D^{\prime}$ is a convex set, it follows that $f(u)=\sum_{0 \leq i \leq n} \mu_{i} f\left(w_{i}\right) \in C^{\prime} \backslash D^{\prime}$. Now consider the second case. Let $P^{\prime}$ be a support affine subspace for the face $D^{\prime}$ and thus, $P^{\prime} \cap C^{\prime}=D^{\prime}$. For $i>k$, we have $f\left(w_{i}\right) \notin D^{\prime}$, and thus, $f\left(w_{i}\right) \notin P^{\prime}$. Put $\nu_{0}=\sum_{0 \leq i \leq k} \mu_{i}, \quad \nu_{1}=$ $\sum_{k+1 \leq i \leq n} \mu_{i}$ and note that $\nu_{0}>0, \nu_{1}>0$ and $\nu_{0}+\nu_{1}=1$. Consider the points $u_{0}^{\prime}=\sum_{0 \leq i \leq k} \frac{\mu_{i}}{\nu_{0}} f\left(w_{i}\right), u_{1}^{\prime}=\sum_{k+1 \leq i \leq n} \frac{\mu_{i}}{\nu_{1}} f\left(w_{i}\right)$. Since $f\left(w_{i}\right) \in D^{\prime}$, for $0 \leq i \leq k$ and $f\left(w_{i}\right) \in C^{\prime} \backslash D^{\prime}$, for $k+1 \leq i \leq n$, it follows that $u_{0}^{\prime} \in D^{\prime} \subseteq P^{\prime}$ and $u_{1}^{\prime} \in C^{\prime} \backslash D^{\prime}$. Also note that $f(u)=\nu_{0} u_{0}^{\prime}+\nu_{1} u_{1}^{\prime}$. If we assume that $f(u) \in D^{\prime} \subseteq P^{\prime}$, it would follow that $u_{1}^{\prime}=\frac{1}{\nu_{1}}\left(f(u)-\nu_{0} u_{0}^{\prime}\right) \in P^{\prime}$. Since $u_{1}^{\prime} \in C^{\prime}$, we would have $u_{1}^{\prime} \in P^{\prime} \cap C^{\prime}=D^{\prime}$, which is a contradiction.

2.2. Convex polytopes $C$ in the Euclidean space $\mathbb{R}^{n}$ are compact metric spaces (see $[2$, Corollary 2.9] or $[9,6.2 .2]$ ). If the geometric $\operatorname{dimension} \operatorname{dim} C=$ $n$, i.e., Aff $(C)=\mathbb{R}^{n}$, then the topological dimension of $C$ also equals $n$. Moreover, the geometric interior $\operatorname{Int}(C)$ coincides with the topological interior of $C$ in $\mathbb{R}^{n}$. To see this we need the fact that $C$ is the intersection of those supporting halfspaces of the facets of $C$ which contain $C$ (see ([1, Appendix II, $\S 4$, Theorem V] or [2, Theorem 4.5]). Since each facet is the intersection of $C$ with the corresponding supporting hyperplane, it follows that $\operatorname{Int}(C)$ is the intersection of the corresponding open halfspaces. However, these are open sets in $\mathbb{R}^{n}$ and their number is finite. Therefore, $\operatorname{Int}(C)$ is an open subset of $\mathbb{R}^{n}$, contained in $C$. Clearly, it is the maximal open subset of $\mathbb{R}^{n}$ contained in $C$, because every neighborhood of a point of $\operatorname{Bd}(C)$ meets $\mathbb{R}^{n} \backslash C$. It follows immediately that the geometric boundary $\mathrm{Bd}(C)$ coincides with the topological boundary of $C$ in $\mathbb{R}^{n}$.

Every $n$-dimensional polytope $C$ in $\mathbb{R}^{n}$ is homeomorphic to the unit $n$-ball $B^{n} \subseteq \mathbb{R}^{n}([1$, Appendix II, $\S 2$, Theorem X $])$. It follows that $n$-dimensional convex polytopes $C \subseteq \mathbb{R}^{n}$ are $n$-manifolds with boundary, the boundary being $\mathrm{Bd} C$. This justifies referring to $n$-dimensional convex polytopes in $\mathbb{R}^{n}$ as to $n$-dimensional cells, shorter $n$-cells in $\mathbb{R}^{n}$.

2.3. If $V$ is an arbitrary vector space, then every $n$-dimensional linear subspace $L$ of $V$ admits a unique topology, called Euclidean, which makes it a topological vector space. To define it, one considers the standard basis $\left\{e_{1}, \ldots, e_{n}\right\}$ of the vector space $\mathbb{R}^{n}, e_{1}=(1,0, \ldots, 0), \ldots, e_{n}=(0, \ldots, 0,1)$, and one considers a basis $\left\{v_{1}, \ldots, v_{n}\right\}$ of the vector space $L$. Let $\varphi: \mathbb{R}^{n} \rightarrow L$ be the linear mapping determined by putting $\varphi\left(e_{i}\right)=v_{i}, 1 \leq i \leq n$. Note that $\varphi$ is an isomorphism of vector spaces. The Euclidean topology on $L$ is the topology which makes $\varphi$ a homeomorphism (see [3, Appendix I. A, Theorem 2.1]). Clearly, $L$ endowed with the Euclidean topology is isomorphic to the Euclidean vector space $\mathbb{R}^{n}$ as a topological vector space. 
One endows $n$-dimensional affine manifolds $M$ in $V$ with a topology, called Euclidean. If $M=v+L_{M}$, where $v \in V$ and $L_{M}$ is an $n$-dimensional linear subspace of $V$ and $\psi: M \rightarrow L$ is the affine isomorphism, given by $\psi(x)=x-v$, then the Euclidean topology on $M$ is the topology which makes $\psi$ a homeomorphism. Affine mappings $f: M \rightarrow N=w+L_{N}$ between finitedimensional affine manifolds are continuous (Euclidean topologies). Indeed, if $\psi_{M}: M \rightarrow L_{M}$ and $\psi_{N}: N \rightarrow L_{N}$ are affine isomorphisms, given by $\psi_{M}(x)=$ $x-v, \psi_{N}(y)=y-w$ and $\varphi: L_{M} \rightarrow L_{n}$ is a linear mapping such that $\varphi \psi_{M}=\psi_{N} f$, then continuity of $f$ is an immediate consequence of the fact that $\varphi$ (just as any linear mapping between finite-dimensional vector spaces) is continuous and $\psi_{M}$ and $\psi_{N}$ are homeomorphisms.

Statements made in Subsection 2.2 about $n$-dimensional convex polytopes in $\mathbb{R}^{n}$ are also valid for $n$-dimensional convex polytopes $C$ in $V$ and their affine hulls Aff $(C)$ in $V$. In particular, $C$ is a closed subset of $\operatorname{Aff}(C)$.

2.4. It is well-known that for non-empty subsets $A, B \subseteq V$, Aff $(A+B)=$ $\operatorname{Aff}(A)+\operatorname{Aff}(B)$ and $\operatorname{Conv}(A+B)=\operatorname{Conv}(A)+\operatorname{Conv}(B)$ (for Conv see $[9$, Theorem 3.2.6]; a proof for Aff is obtained from the proof of $[9$, Theorem 3.1.4] by obvious modifications). An immediate consequence is the fact that the sum of two affine manifolds, two convex sets or two convex polytopes in $V$ is an affine manifold, a convex set or a convex polytope (see [9, Theorem $6.2 .7])$.

If $\left\{a_{i} \mid 1 \leq i \leq k\right\}$ and $\left\{b_{j} \mid 1 \leq j \leq l\right\}$ are the sets of vertices of convex polytopes $A$ and $B$ in $V$, then $A+B=\operatorname{Conv}\left(\left\{a_{i}+b_{j} \mid 1 \leq i \leq k, 1 \leq j \leq l\right\}\right)$ (see [9, Theorem 6.2.7]). It follows that every vertex of the polytope $A+B$ is of the form $a_{i}+b_{j}$ (see [2, Theorem 7.2]). In general, the converse does not hold, i.e., some of the points $a_{i}+b_{j}$ may not be vertices of $A+B$. E.g., if $A=[0,1] \subseteq \mathbb{R}, B=[0,2] \subseteq \mathbb{R}$, then the points $1+0=1$ and $0+2=2$ are not vertices of $A+B=[0,3]$. However, the converse does hold if the affine hulls of the two convex polytopes are affinely independent (see Subsections 2.5 and 2.6 ).

2.5. Two affine manifolds $M=v+L_{M}$ and $N=w+L_{N}$ are affinely independent if the linear subspaces $L_{M}$ and $L_{N}$ of $V$ are linearly independent, i.e., $L_{M} \cap L_{N}=0$. In that case $M+N=(v+w)+\left(L_{M}+L_{N}\right)$ and thus, $L_{M+N}=L_{M}+L_{N}$ and $\operatorname{dim}(M+N)=\operatorname{dim} L_{M+N}=\operatorname{dim}\left(L_{M}+L_{N}\right)=$ $\operatorname{dim} L_{M}+\operatorname{dim} L_{N}=\operatorname{dim} M+\operatorname{dim} N$. Moreover, every point $z \in M+N$ admits unique points $x \in M, y \in N$ such that $z=x+y$. Indeed, if $x, x^{\prime} \in M$ and $y, y^{\prime} \in N$, then $z=x+y=x^{\prime}+y^{\prime}$ implies $x-x^{\prime}=y^{\prime}-y$. Since $x-x^{\prime} \in L_{M}$, $y^{\prime}-y \in L_{N}$, it follows that $x-x^{\prime}=y^{\prime}-y$ and thus, $x-x^{\prime}=0=y-y^{\prime}$. We refer to the functions $p_{M}=M+N \rightarrow M$ and $p_{N}=M+N \rightarrow N$, given by $p_{M}(x+y)=x$ and $p_{N}(x+y)=y$ as to canonical projections. It is readily verified that $p_{M}$ and $p_{N}$ are affine mappings. Therefore, they are continuous. 
2.6. The following lemma plays an important role in the paper.

LemMa 2.2. Let $A$ and $B$ be convex polytopes in a vector space $V$. If their affine hulls $\mathrm{Aff}(A)$ and $\mathrm{Aff}(B)$ are affinely independent, then the following assertions hold:

(i) $\operatorname{dim}(A+B)=\operatorname{dim} A+\operatorname{dim} B$.

(ii) The faces of $A+B$ are convex polytopes of the form $A^{\prime}+B^{\prime}$, where $A^{\prime} \leq A$ and $B^{\prime} \leq B$

(iii) The vertices of $A+B$ are the points $a_{i}+b_{j}$, where $a_{i}$ ranges over the set of vertices of $A$ and $b_{j}$ ranges over the set of vertices of $B$.

(iv) If $A^{\prime}, A^{\prime \prime} \subseteq A$ and $B^{\prime}, B^{\prime \prime} \subseteq B$, then $\left(A^{\prime}+B^{\prime}\right) \cap\left(A^{\prime \prime}+B^{\prime \prime}\right)=\left(A^{\prime} \cap\right.$ $\left.A^{\prime \prime}\right)+\left(B^{\prime} \cap B^{\prime \prime}\right)$.

Proof. $(i)$. Put $M=\operatorname{Aff}(A)$ and $N=\operatorname{Aff}(B)$ and note that Aff $(A+$ $B)=\operatorname{Aff}(A)+\operatorname{Aff}(B)=M+N$. Therefore, $\operatorname{dim}(A+B)=\operatorname{dim} \operatorname{Aff}(A+B)=$ $\operatorname{dim}(M+N)$. As seen in 2.5, $\operatorname{dim}(M+N)=\operatorname{dim} M+\operatorname{dim} N=\operatorname{dim} A+\operatorname{dim} B$.

(iv). If $z \in\left(A^{\prime}+B^{\prime}\right) \cap\left(A^{\prime \prime}+B^{\prime \prime}\right)$, then there are points $a^{\prime} \in A^{\prime} \subseteq A$, $b^{\prime} \in B^{\prime} \subseteq B, a^{\prime \prime} \in A^{\prime \prime} \subseteq A, b^{\prime \prime} \in B^{\prime \prime} \subseteq B$ such that $z=a^{\prime}+b^{\prime}=a^{\prime \prime}+b^{\prime \prime}$. Since $a^{\prime}, a^{\prime \prime} \in A \subseteq M$ and $b^{\prime}, b^{\prime \prime} \in B \subseteq N$, it follows that $a^{\prime}=a^{\prime \prime} \in A^{\prime} \cap A^{\prime \prime}$ and $b^{\prime}=b^{\prime \prime} \in B^{\prime} \cap B^{\prime \prime}$ (see 2.5) and thus, $z \in\left(A^{\prime} \cap A^{\prime \prime}\right)+\left(B^{\prime} \cap B^{\prime \prime}\right)$. The converse implication is obvious.

(iii) is an immediate consequence of $(i i)$. To prove $(i i)$, consider the following statement.

$(\text { ii })^{\prime}$. The facets of $A+B$ are convex polytopes of the form $A^{\prime}+B$ or $A+B^{\prime}$, where $A^{\prime}$ ranges over the facets of $A$ and $B^{\prime}$ ranges over the facets of $B$.

$(i i)^{\prime} \Rightarrow(i i)$. If $A^{\prime}$ is a facet of $A$, then $\operatorname{Aff}\left(A^{\prime}\right) \subseteq \operatorname{Aff}(A)$ and thus, Aff $\left(A^{\prime}\right)$ and Aff $(B)$ are affinely independent. This enables us to apply $(i i)^{\prime}$ to $A^{\prime}+B$ and conclude that, for facets $A^{\prime \prime}$ of $A$ and $B^{\prime}$ of $B$, the faces $A^{\prime \prime}+B$ and $A^{\prime}+B^{\prime}$ are facets of $A^{\prime}+B$. Analogously, applying $(i i)^{\prime}$ to $A+B^{\prime}$, one concludes that, for facets $A^{\prime}$ of $A$ and $B^{\prime \prime}$ of $B^{\prime}$, the faces $A^{\prime}+B^{\prime}$ and $A+B^{\prime \prime}$ are facets of $A+B^{\prime}$. Since descending chains of facets yield all faces of a convex polytope, one obtains $(i i)$.

Proof of $(i i)^{\prime}$. Let $M=v+L_{M}, N=w+L_{N}$, where $v \in M, w \in N$ and $L_{M}, L_{N}$ are linear subspaces of $V$ such that $L_{M} \cap L_{N}=0$. Consider the affine polytopes $A^{*}=A-v \subseteq L_{M}$ and $B^{*}=B-w \subseteq L_{N}$ and note that $A^{*}+B^{*}=A+B-(v+w)$. Since translation preserves the face structure of polytopes, it suffices to prove $(i i)^{\prime}$ for $A^{*}+B^{*}$. This shows that there is no loss of generality in assuming from the beginning that $\operatorname{Aff}(A)=L_{M}$ and $\operatorname{Aff}(B)=L_{N}$ are linear subspaces of $V, \operatorname{dim} L_{M}=m, \operatorname{dim} L_{N}=n$ and $L_{M} \cap L_{N}=0$. Choosing a basis $\left\{v_{1}, \ldots, v_{m}\right\}$ of $L_{M}$ and a basis $\left\{w_{1}, \ldots, w_{n}\right\}$ of $L_{N}$, one obtains linear isomorphisms $\varphi_{M}: \mathbb{R}^{m} \rightarrow L_{M}$ and $\varphi_{N}: \mathbb{R}^{n} \rightarrow L_{N}$ as in 2.3. Clearly, these isomorphisms induce an isomorphism $\varphi: \mathbb{R}^{m+n} \rightarrow$ $L_{M}+L_{N}$. Since linear isomorphisms preserve the face structure of convex 
polytopes, it suffices to prove $(i i)^{\prime}$ in the case when $A \subseteq \mathbb{R}^{m} \times 0, B \subseteq 0 \times \mathbb{R}^{n}$, $\operatorname{dim} A=m$ and $\operatorname{dim} B=n$. Note that in that case, for arbitrary subsets $D \subseteq \mathbb{R}^{M} \times 0$ and $E \subseteq 0 \times \mathbb{R}^{n}$, one has $D+E=D \times E \subseteq \mathbb{R}^{m+n}=\mathbb{R}^{m} \times \mathbb{R}^{n}$, because $(d, 0)+(0, e)=(d, e)$, for $d \in \mathbb{R}^{m}, e \in \mathbb{R}^{n}$. In particular, $A+B=$ $A \times B \subseteq \mathbb{R}^{m+n}$.

Assume that $A^{\prime}<A$ is a facet of $A$, i.e., an $(m-1)$-dimensional face of $A$. Let $H \subseteq \mathbb{R}^{m}$ be the supporting halfspace of $A$, whose supporting hyperplane $S \subseteq H$ intersect $A$ in the facet $A^{\prime}$. Then $K=H \times \mathbb{R}^{n} \subseteq \mathbb{R}^{m+n}$ is a supporting halfspace of $A \times B$, whose supporting hyperplane $T=S \times \mathbb{R}^{n}$ intersects $A \times B$ in the set $T \cap(A \times B)=\left(S \times \mathbb{R}^{n}\right) \cap(A \times B)=A^{\prime} \times B$, because $S \times A=A^{\prime}$. Since $\operatorname{dim}\left(A^{\prime} \times B\right)=m-1+n$, we see that $A^{\prime}+B=A^{\prime} \times B$ is a facet of $A+B=A \times B$. In the same way one concludes that $A+B^{\prime}$ is a facet of $A+B$, whenever $B^{\prime}$ is a facet of $B$. It remains to show that in this way we have obtained all the facets of $A+B$.

Recall that the union of all facets $A^{\prime}$ of $A$ coincides with the geometric boundary $\operatorname{Bd}(A)$ and thus, the union $\bigcup_{A^{\prime}}\left(A^{\prime}+B\right)=\left(\bigcup_{A^{\prime}} A^{\prime}\right)+B=\operatorname{Bd}(A)+$ $B$. Similarly, $\bigcup_{B^{\prime}}\left(A+B^{\prime}\right)=A+\operatorname{Bd}(B)$. It follows that the union of all the facets we have just constructed equals $(\operatorname{Bd}(A)+B) \cup(A+(\operatorname{Bd}(B))$. For the topological boundary, the following formula is easily proved: $(\operatorname{Bd}(A) \times B) \cup$ $(A \times \mathrm{Bd}(B))=\mathrm{Bd}(A \times B)$. It suffices to note that, for open sets $U_{M}$ in $\mathbb{R}^{m}$ and $U_{N}$ in $\mathbb{R}^{n}$, the set $U_{M} \times U_{N}$ is open in $\mathbb{R}^{m} \times \mathbb{R}^{n}=\mathbb{R}^{m+n}$. Since $D+E=D \times E$, the boundary formula can be written in the form $\operatorname{Bd}(A+B)=$ $(\mathrm{Bd}(A)+B) \cup(A+(\mathrm{Bd}(B))$. However, for convex polytopes, the topological boundary coincides with the geometric boundary. Consequently, interpreting $\mathrm{Bd}$ in the boundary formula as the geometric boundary, we conclude that the facets of $A+B$, which we have constructed above, cover the whole geometric boundary of $A+B$ and thus, there can be no other facets of $A+B$.

2.7. In the present paper we find it convenient to use the notion of a direct sum $M \oplus N$ of two affine manifolds in $V$ and the notion of a direct sum $A \oplus B$ of two convex polytopes in $V$. We define $M \oplus N$ only when the affine manifolds $M$ and $N$ intersect in a single point $w$, which implies that they are affinely independent. By definition, $M \oplus N=M+N-w$. Note that the point $w$ is completely determined by $M$ and $N$ and we call it the reference point of the direct sum $M \oplus N$ (in [7] we called it base-point). Being the translate of the affine manifold $M+N$, the direct sum $M \oplus N$ is also an affine manifold. By Subsection 2.5, $\operatorname{dim}(M \oplus N)=\operatorname{dim}(M+N)=\operatorname{dim} M+\operatorname{dim} N$. Since $M$ and $N$ are affinely independent, the canonical projections $p_{M}=M \oplus N \rightarrow M$ and $p_{N}=M \oplus N \rightarrow N$ are well-defined affine, hence also continuous mappings.

Analogously, if $A, B$ are convex polytopes in $V$ such that Aff $(A) \cap \operatorname{Aff}(B)$ is a single point $w \in V$, we define (see [9, Definition 6.4.3]) $A \oplus B$ by the formula

$$
A \oplus B=A+B-w .
$$


We call $w$ the reference point of $A \oplus B$.

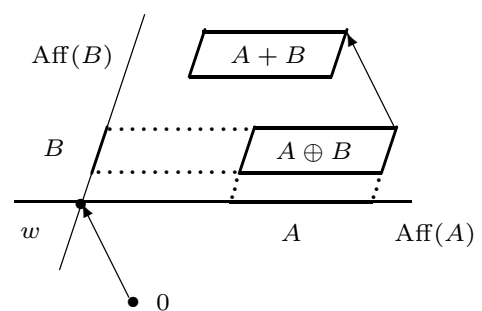

Figure 1. Sum $A+B$ and direct sum $A \oplus B$

Being a translate of the convex polytope $A+B$, the direct sum $A \oplus B$ is a convex polytope. Moreover, by Lemma $2.2, \operatorname{dim}(A \oplus B)=\operatorname{dim}(A+B)=$ $\operatorname{dim} A+\operatorname{dim} B$, the faces of $A \oplus B$ are convex polytopes of the form $A^{\prime}+B^{\prime}-w$, where $A^{\prime} \leq A$ and $B^{\prime} \leq B$. The vertices of $A \oplus B$ are the points $a_{i}+b_{j}-w$, where $a_{i}$ ranges over the set of vertices of $A$ and $b_{j}$ ranges over the set of vertices of $B$. If $A^{\prime}, A^{\prime \prime} \subseteq A$ and $B^{\prime}, B^{\prime \prime} \subseteq B$, then $\left(A^{\prime}+B^{\prime}-w\right) \cap\left(A^{\prime \prime}+\right.$ $\left.B^{\prime \prime}-w\right)=\left(A^{\prime} \cap A^{\prime \prime}\right)+\left(B^{\prime} \cap B^{\prime \prime}\right)-w$. In general, for faces $A^{\prime} \leq A$ and $B^{\prime} \leq B$, the direct sum $A^{\prime} \oplus B^{\prime}$ is not defined, because $\operatorname{Aff}\left(A^{\prime}\right) \cap \operatorname{Aff}\left(B^{\prime}\right)$ can be empty. Clearly, $A^{\prime} \oplus B^{\prime}$ is defined if and only if $\operatorname{Aff}\left(A^{\prime}\right) \cap \operatorname{Aff}\left(B^{\prime}\right) \neq \emptyset$. In that case $\operatorname{Aff}\left(A^{\prime}\right) \cap \operatorname{Aff}\left(B^{\prime}\right)=\{w\}$ and $A^{\prime} \oplus B^{\prime}=A^{\prime}+B^{\prime}-w$. Note that the direct sum $A \oplus B$ is invariant with respect to translations, because $\operatorname{Aff}(A) \cap \operatorname{Aff}(B)=\{w\}$ implies $\operatorname{Aff}(A+t) \cap \operatorname{Aff}(B+t)=\{w+t\}$ and thus, $(A+t) \oplus(B+t)=(A+B+2 t)-(w+t)=A+B-w+t=(A \oplus B)+t$. This is not the case with the ordinary sum, because, $(A+t)+(B+t) \neq(A+B)+t$, for $t \neq 0$.

2.8. If $M \oplus N$ and $M^{\prime} \oplus N^{\prime}$ are direct sums of affine manifolds with reference points $w$ and $w^{\prime}$, respectively, then mappings $f: M \rightarrow M^{\prime}$ and $g: N \rightarrow N^{\prime}$ induce a mapping $f \oplus g: M \oplus N \rightarrow M^{\prime} \oplus N^{\prime}$. By definition, if $z=x+y-w \in M \oplus N, x \in M, y \in N$, then $(f \oplus g)(z)=f(x)+g(y)-w^{\prime}$. A straightforward verification shows that, for affine mappings $f$ and $g$, the mapping $f \oplus g$ is also an affine mapping. Moreover, $\left(f^{\prime} \oplus g^{\prime}\right)(f \oplus g)=\left(f^{\prime} f \oplus g^{\prime} g\right)$ and $1 \oplus 1=1$. Consequently, if $f$ and $g$ are affine isomorphisms, then so is $f \oplus g$. For direct sums $M \oplus N$ with reference point $w$ and for points $x \in M$ and $y \in N$, it is sometimes convenient to denote $x+y-w$ by $x \oplus y$. Then $f \oplus g$ can be defined by the simple formula $(f \oplus g)(x \oplus y)=f(x) \oplus g(y)$. However, one can use this shorter notation only when it is clear about which direct sum $M \oplus N$ and $M^{\prime} \oplus N^{\prime}$ we speak, because for arbitrary points $x, y \in V$, the set $\{x\} \oplus\{y\}$ is defined only when Aff $\{x\} \cap \operatorname{Aff}\{y\}=\{x\} \cap\{y\} \neq \emptyset$ and thus, $x=y$.

Since a convex polytope is the convex hull of the set of its vertices, for convex polytopes $A \oplus B$ with reference point $w$ and $A^{\prime} \oplus B^{\prime}$ with reference point 
$w^{\prime}$ and for affine mappings $f: \operatorname{Aff}(A) \rightarrow \operatorname{Aff}\left(A^{\prime}\right)$ and $g: \operatorname{Aff}(B) \rightarrow \operatorname{Aff}\left(B^{\prime}\right)$, the affine mapping $f \oplus g: \operatorname{Aff}(A) \oplus \operatorname{Aff}(B) \rightarrow \operatorname{Aff}\left(A^{\prime}\right) \oplus \operatorname{Aff}\left(B^{\prime}\right)$ is completely determined by its values at the vertices of $a_{i}+b_{j}-w$ of $A \oplus B$, i.e., by the values $f\left(a_{i}\right)+g\left(b_{j}\right)-w^{\prime}$.

2.9. We now endow the vector space $V$ with the topology generated by the Euclidean topologies of the finite-dimensional affine manifolds $M$ in $V$ and call this topology the finite topology of $V$. By definition, a subset $H \subseteq V$ is closed in $V$ provided $H \cap M$ is closed in $M$ (Euclidean topology), for every finite-dimensional affine manifold $M$ in $V$ (see [3, Appendix I. A, Definition $4.2]$ ). Note that affine manifolds in $\mathbb{R}^{n}$ are closed subsets of $\mathbb{R}^{n}$. Therefore, affine manifolds in finite-dimensional affine manifolds $M$ in $V$ are closed in $M$ (Euclidean topology). Since the intersection $M \cap N$ of an affine manifold $M$ in $V$ with a finite-dimensional affine manifold $N$ in $V$ is a finite-dimensional affine manifold in $N$, it follows that $M \cap N$ is closed in $N$, which proves that every affine manifold $M$ in $V$ is closed in $V$. Since every convex polytope $C$ is closed in its affine hull Aff $(C)$, it follows that $C$ is also closed in $V$. It is known that $V$ endowed with the finite topology as well as all of its subspaces are (Hausdorff) paracompact spaces. In general, $V$ is not a topological vector space (see [3, Appendix I. A, 4.3]).

2.10. In this paper by a cellular complex in a vector space $V$ we mean a collection $L$ of convex polytopes $c \subseteq V$, called cells of $L$, such that all faces $c^{\prime}$ of a cell $c \in L$ belong to $L$ and any two cells $c^{\prime}, c^{\prime \prime} \in L$ intersect in a common (possibly empty) face of $c^{\prime}$ and $c^{\prime \prime}$. We allow $L$ to be infinite, but we require that, for any convex polytope $C \subseteq V$, there are only finitely many different intersections $C \cap c$, when $c$ ranges over $L$. By a morphism of cellular complexes $\vartheta: L \rightarrow N$ we mean a sequence of functions $\vartheta_{k}: L_{k} \rightarrow N_{k}$, $k \in\{0,1, \ldots\}$, between the sets of $k$-cells $L_{k}$ of $L$ and $N_{k}$ of $N$, which preserve faces, i.e., if $c^{\prime} \in L_{k^{\prime}}, c \in L_{k}$ and $c^{\prime} \leq c$, then $\vartheta_{k^{\prime}}\left(c^{\prime}\right) \leq \vartheta_{k}(c)$. An isomorphism of cellular complexes is a morphism $\vartheta: L \rightarrow N$, which admits an inverse, i.e., there is a morphism of cellular complexes $\varphi: N \rightarrow L$ such that $\varphi_{k} \vartheta_{k}=$ id and $\vartheta_{k} \varphi_{k}=\mathrm{id}$, for every $k$.

The carrier $|L|$ of a cellular complex $L$ in $V$ is the union of all cells belonging to $L$. The finite topology on $V$ induces a topology on $|L|$, called the finite topology of $|L|$. It coincides with the CW-topology of $|L|$, i.e., the topology generated by the Euclidean topologies of the cells $c \in L$, which implies that our cellular complexes are special cases of CW-complexes. Indeed, let $H \subseteq|L|$ be a set closed in the CW-topology of $|L|$, i.e., let $H \cap c$ be closed in $c$, for every $c \in L$. To show that $H$ is also closed in the finite topology of $|L|$, it suffices to show that $H \cap F$ is closed in $F$, for every finite-dimensional affine manifold $F$ of $V$, endowed with the Euclidean topology. Since the topology of $\mathbb{R}^{n}$ is generated by the topologies of convex polytopes contained in $\mathbb{R}^{n}$, the topology of $F$ is generated by the topologies of convex polytopes contained in 
$F$. Therefore, it suffices to show that $H \cap C$ is closed in $C$, for every convex polytope $C \subseteq F$. By the definition of a cellular complex, there are finitely many cells $c_{1}, \ldots, c_{k} \in L$ such that $C=\left(C \cap c_{1}\right) \cup \ldots \cup\left(C \cap c_{k}\right)$ and thus, $H \cap C=\left(H \cap C \cap c_{1}\right) \cup \ldots \cup\left(H \cap C \cap c_{k}\right)$. By assumption, $H \cap c_{i}$ is closed in $c_{i}$ and thus, $H \cap C \cap c_{i}$ is closed in $C \cap c_{i}$. Since $c_{i}$ is closed in $V$ (finite topology), it follows that $C \cap c_{i}$ is closed in $C$, hence also $H \cap C \cap c_{i}$ is closed in $C$. However, $H \cap C$ is a finite union of sets of the form $H \cap C \cap c_{i}$ and therefore, $H \cap C$ is closed in $C$. Conversely, sets $H \subseteq|L|$, closed in the finite topology of $|L|$ are closed in the CW-topology of $|L|$, because for $E=\operatorname{Aff}(c)$, the set $H \cap E$ is closed in $E$ and thus, $H \cap c$ is closed in $c$.

A cellular complex $L$ in $V$ is a subdivision of a cellular complex $K$ provided every cell $c \in L$ is contained in a cell $d \in K$ and every cell $d \in K$ is the union of a collection of cells $c \in L$. By our definition of a cellular complex, the collection of all cells $c \in L$ contained in a cell $d \in K$ is a finite cellular complex $L(d)$ with carrier $|L(d)|=d$ (we use the same notation $d$ for the cell $d$, for the complex consisting of $d$ and its faces, as well as for its carrier $|d|)$.

REMARK 2.3. It appears that complexes consisting of convex polytopes are not used very often in recent literature. However, in older literature they were standard objects (see e.g., [1, Chapter III, §1]).

Simplicial complexes in $V$ are special cases of cellular complexes, whose $n$ cells $\sigma$, called simplices, are convex hulls of sets consisting of $n+1$ affinely independent points $v_{0}, \ldots, v_{n}$ of $V$, called vertices of $\sigma, n \in\{0,1, \ldots\}$. Throughout this paper, for simplices in $V$, we use the standard notation $\sigma=\left[v_{0}, \ldots, v_{n}\right]$. Note that the order of the vertices is irrelevant.

Every abstract simplicial complex $K$ admits a geometric realization in a vector space $V$ as a simplicial complex $L$ in $V$. It suffices to take for $V$ a vector space, whose basis $\left\{a_{i}\right\}$ admits a bijection onto the set $\left\{v_{i}\right\}$ of all vertices of $K$. With every abstract simplex $\left\{v_{i_{0}}, \ldots, v_{i_{k}}\right\}$ of $K$ one then associates the polytope (simplex) $\left[a_{i_{0}}, \ldots, a_{i_{k}}\right]$ in $V$. It is readily seen that the simplices $c=\left[a_{i_{0}}, \ldots, a_{i_{k}}\right]$ form a simplicial complex $L$, the geometric realization of the complex $K$. Note that, for any convex polytope $C \subseteq V$, there are only finitely many different intersections $C \cap c$, when $c$ ranges over $L$.

\section{The First CANONICAL SUbDIVISION $L(K)$}

3.1. To construct the cellular subdivision $L(K)$ of a simplicial complex $K$, we assume that $K$ is contained in a vector space $V$. We first construct the cellular subdivisions $L(\sigma)$ of $\sigma$, for all simplices $\sigma \in K$. If $\operatorname{dim} \sigma=n$, it suffices to define the $n$-cells of $L(\sigma)$. Then $L(\sigma)$ consists of these $n$-cells and of all of their faces (including the empty face). The construction of $L(\sigma)$ will be done in such a way that the intersections of its cells with any proper face $\zeta<\sigma$ coincides with $L(\zeta)$. Therefore, the union of all $L(\sigma)$, when $\sigma$ 
ranges over $K$, is a cellular subdivision of $K$. By definition, this is the desired subdivision $L(K)$ of $K$.

Among the $n$-cells of $L(\sigma)$, where $\operatorname{dim} \sigma=n$, there is one, contained in the interior $\operatorname{Int}(\sigma)$ of $\sigma$. This $n$-cell is actually an $n$-simplex, which we call the central $n$-simplex of $\sigma$ and we denote it by $c^{\sigma}$. All other $n$-cells of $L(\sigma)$ meet the boundary $\operatorname{Bd}(\sigma)$ (also denoted by $\partial \sigma$ ) and are called peripheral $n$-cells of $\sigma$. If $v_{0}, \ldots, v_{n}$ are the vertices of $\sigma$ (repetition is not allowed), we use the notation $\sigma=\left[v_{0}, \ldots, v_{n}\right]$. Each peripheral $n$-cell of $\sigma$ is determined by a proper face $\tau<\sigma$, i.e., $\tau=\left[v_{i_{0}}, \ldots, v_{i_{k}}\right]$, where $0 \leq k<n$, $\left\{i_{0}, \ldots, i_{k}\right\} \subset\{0, \ldots, n\}, i_{0}<\ldots<i_{k}$, and by a permutation $\pi$ of the complement $\left\{j_{k+1}, \ldots, j_{n}\right\}=\{0, \ldots, n\} \backslash\left\{i_{0}, \ldots, i_{k}\right\}, j_{k+1}<\ldots<j_{n}$. The corresponding $n$-cell is denoted by $c_{\pi}^{\tau}$. The central $n$-simplex $c^{\sigma}$ can also be viewed as an $n$-cell of the form $c_{\pi}^{\tau}$, where $\tau=\sigma$ and $\pi$ is the empty permutation (this explains the notation $c^{\sigma}$ ). Arguments for peripheral $n$-cells given throughout this paper readily adapt to the central simplex and are omitted in most cases.

3.2. To define the central $n$-simplex $c^{\sigma}$ of $\sigma=\left[v_{0}, \ldots, v_{n}\right]$, we consider the barycenter $b^{\sigma}$ of $\sigma$,

$$
b^{\sigma}=\frac{1}{n+1} \sum_{i=0}^{i=n} v_{i}
$$

and the homothetic transformation $\chi^{\sigma}: \sigma \rightarrow \sigma$ with center $b^{\sigma}$ and ratio $\frac{1}{2}$, i.e., we put $\chi^{\sigma}(x)-b^{\sigma}=\frac{1}{2}\left(x-b^{\sigma}\right)$, for $x \in \sigma$. By definition, $c^{\sigma}=\chi^{\sigma}(\sigma)$. Clearly, $c^{\sigma}$ is the $n$-dimensional simplex $\left[w_{0}^{\sigma}, \ldots, w_{n}^{\sigma}\right]$ with vertices $w_{i}^{\sigma}=\chi^{\sigma}\left(v_{i}\right), 0 \leq$ $i \leq n$. Since $w_{i}^{\sigma} \in \operatorname{Int}(\sigma)$, it follows that $c^{\sigma} \subseteq \operatorname{Int}(\sigma)$. In situations where it is clear that we are performing a construction within a simplex $\sigma=\left[v_{0}, \ldots, v_{n}\right]$, we will often be using the convenient (shorter) notation $b^{0 \ldots n}$ for $b^{\sigma}, c^{0 \ldots n}$ for $c^{\sigma}$ and $w_{i}^{0 \ldots n}$ for $w_{i}^{\sigma}$, where the upper index $\sigma$ is replaced by the indices of the vertices of $\sigma$. Consequently,

$$
\begin{gathered}
w_{i}^{\sigma}=w_{i}^{0 \ldots n}=\frac{1}{2}\left(v_{i}+b^{0 \ldots n}\right)=\frac{1}{2} v_{i}+\frac{1}{2(n+1)} \sum_{j=0}^{j=n} v_{j}, 0 \leq i \leq n, \\
c^{\sigma}=c^{0 \ldots n}=\left[w_{0}^{0 \ldots n}, \ldots, w_{n}^{0 \ldots n}\right]=\left[w_{0}^{\sigma}, \ldots, w_{n}^{\sigma}\right] .
\end{gathered}
$$

Note that $b^{0}=v_{0}=w_{0}^{0}$ and $c^{0}=\left[w_{0}^{0}\right]$. Also note that the order of the upper indices in $b^{0 \ldots n}, w_{i}^{0 \ldots n}$ and $c^{0 \ldots n}$ is irrelevant. The convenient notation is also used in the case of a face $\tau=\left[v_{i_{0}}, \ldots, v_{i_{k}}\right]$ of $\sigma=\left[v_{0}, \ldots, v_{n}\right]$. E.g., the corresponding central $k$-simplex $c^{\tau}$ of $\tau$ will also be denoted by $c^{i_{0} \ldots i_{k}}$.

REMARK 3.1. It is useful to keep in mind that $w_{i}^{r \ldots s}=w_{i^{\prime}}^{r^{\prime}} \ldots s^{\prime}$ if and only if $\{r, \ldots, s\}=\left\{r^{\prime}, \ldots, s^{\prime}\right\}$ and $i=i^{\prime}$. Indeed, $w_{i}^{r \ldots s} \in \operatorname{Int}\left[v_{r}, \ldots, v_{s}\right]$ and $w_{i^{\prime}}^{r^{\prime}} \ldots s^{\prime} \in \operatorname{Int}\left[v_{r^{\prime}}, \ldots, v_{s^{\prime}}\right]$. Therefore, $w_{i}^{r \ldots s}=w_{i^{\prime}}^{r^{\prime}} \ldots s^{\prime}$ implies that the interiors of the faces $\left[v_{r}, \ldots, v_{s}\right]$ and $\left[v_{r^{\prime}}, \ldots, v_{s^{\prime}}\right]$ of the simplex $\left[v_{0}, \ldots, v_{n}\right]$ intersect. This is possible only if the two faces agree, i.e., $\left[v_{r}, \ldots, v_{s}\right]=\left[v_{r^{\prime}}, \ldots, v_{s^{\prime}}\right]$ 
and thus, $\{r, \ldots, s\}=\left\{r^{\prime}, \ldots, s^{\prime}\right\}$. However, by (3.2), $0=w_{i}^{r \ldots s}-w_{i^{\prime}}^{r \ldots s}=$ $\frac{1}{2}\left(v_{i}-v_{i^{\prime}}\right)$ implies $v_{i}=v_{i^{\prime}}$ and thus, $i=i^{\prime}$.

For a point $u \in \sigma=\left[v_{0}, \ldots, v_{n}\right]$, let $\lambda_{i}=\lambda_{i}(u), 0 \leq i \leq n$, denote the barycentric coordinates of $u$ with respect to the vertices $v_{0}, \ldots, v_{n}$ of $\sigma$ (shorter, with respect to $\sigma$ ). Then $u \in \sigma$ belongs to $c^{\sigma}$ if and only if

$$
\lambda_{0}, \ldots, \lambda_{n}(u) \geq \frac{1}{2(n+1)} \text {. }
$$

Indeed, let $\mu_{i}=\mu_{i}(u), 0 \leq i \leq n$, be the barycentric coordinates of $u \in c^{0 \ldots n}$ with respect to the vertices $w_{0}^{0 \ldots n}, \ldots, w_{n}^{0 \ldots \ldots n}$ of $c^{\sigma}$. Substituting in the equality

$$
u=\sum_{j=0}^{j=n} \lambda_{j} v_{j}=\sum_{i=0}^{i=n} \mu_{i} w_{i}^{0 \ldots n}
$$

the values of $w_{i}^{0 \ldots \ldots n}$ from (3.2), one concludes that

$$
\lambda_{j}=\frac{\mu_{j}}{2}+\frac{1}{2(n+1)}, 0 \leq j \leq n .
$$

Now formula (3.4) is a consequence of the fact that $\mu_{j} \geq 0$. Conversely, (3.4) implies $u \in c^{0 \ldots n}$, because the numbers $\mu_{j}$, determined by (3.6), have the property that $\mu_{j} \geq 0, \sum_{j=0}^{j=n} \mu_{j}=1$ and thus, $\mu_{j}$ are the barycentric coordinates of $u$ with respect to $w_{0}^{0 \ldots n}, \ldots, w_{n}^{0 \ldots \ldots n}$.

3.3. By definition, in $L\left[v_{0}\right]$ there are no peripheral 0-cells. In $L\left[v_{0}, v_{1}\right]$ there are two peripheral 1-cells, i.e., the 1 -simplices $\left[w_{0}^{0}, w_{0}^{01}\right]$ and $\left[w_{1}^{1}, w_{1}^{01}\right]$. In $L\left[v_{0}, v_{1}, v_{2}\right]$ there are nine peripheral 2-cells, i.e., the six 2-simplices meeting the boundary of $\left[v_{0}, v_{1}, v_{2}\right]$ and the three parallelograms, shown in Figure 2.

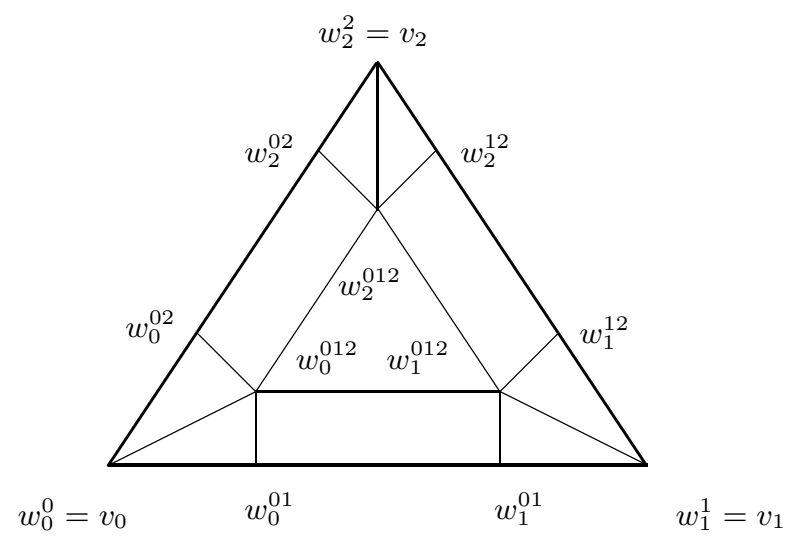

FiguRE 2. The decomposition $L\left[v_{0}, v_{1}, v_{2}\right]$

In general, the peripheral $n$-cell $c_{\pi}^{\tau}$ of $\sigma$ is determined by the central $k$ simplex $c^{\tau}$ of the face $\tau$ of $\sigma$ and by an $(n-k)$-simplex $d_{\pi}^{\tau} \subseteq \sigma$. In what follows 
we will describe $d_{\pi}^{\tau}$ and $c_{\pi}^{\tau}$ in the case when $\tau=\left[v_{0}, \ldots, v_{k}\right], 0 \leq k<n$, and therefore, $\pi$ is a permutation of the set $\{k+1, \ldots, n\}$. In all other cases $d_{\pi}^{\tau}$ and $c_{\pi}^{\tau}$ are obtained by adequately relabelling the vertices of $\sigma$. We will also be using the convenient notation $d_{\pi}^{0 \ldots k}$ for $d_{\pi}^{\tau}$ and $c_{\pi}^{0 \ldots k}$ for $c_{\pi}^{\tau}$.

By definition, $d_{\pi}^{\tau}=d_{\pi}^{0 \ldots k}$ is the $(n-k)$-simplex

$$
d_{\pi}^{0 \ldots k}=\left[w_{0}^{0 \ldots k}, w_{0}^{0 \ldots k \pi(k+1)}, \ldots, w_{0}^{0 \ldots k \pi(k+1) \ldots \pi(k+i)}, \ldots, w_{0}^{0 \ldots n}\right] .
$$

Note that $w_{0}^{0 \ldots k \pi(k+1) \ldots \pi(n)}=w_{0}^{0 \ldots n}$, because $\{\pi(k+1), \ldots, \pi(n)\}=\{k+$ $1, \ldots, n\}$.

The points listed in (3.7) are affinely independent and belong to $\sigma$. Therefore, $d_{\pi}^{0 \ldots \ldots k}$ is an $(n-k)$-simplex contained in $\sigma$. Let us show that even the points in the larger list

$$
w_{0}^{0 \ldots k}, \ldots, w_{k}^{0 \ldots k}, w_{0}^{0 \ldots k \pi(k+1)}, \ldots, w_{0}^{0 \ldots k \pi(k+1) \ldots \pi(k+i)}, \ldots, w_{0}^{0 \ldots n}
$$

are affinely independent. Indeed, consider the affine hulls $S_{k}=\operatorname{Aff}\left\{v_{0}, \ldots, v_{k}\right\}$ and $S_{k+i}=\operatorname{Aff}\left\{v_{0}, \ldots, v_{k}, v_{\pi(k+1)}, \ldots, v_{\pi(k+i)}\right\}, 1 \leq i \leq n-k$. Since $S_{k} \subseteq S_{k+1} \subseteq \ldots \subseteq S_{n}=\operatorname{Aff}(\sigma)$ and the points $w_{0}^{0 \ldots k}, \ldots, w_{k}^{0 \ldots k}$ are affinely independent and belong to the simplex $\left[v_{0}, \ldots, v_{k}\right] \subseteq S_{k}$, it suffices to show that $w_{0}^{0 \ldots k \pi(k+1) \ldots \pi(k+i)} \in S_{k+i} \backslash S_{k+i-1}$, for $1 \leq i \leq n-k$. Indeed, $w_{0}^{0 \ldots k \pi(k+1) \ldots \pi(k+i)} \in c^{0 \ldots k \pi(k+1) \ldots \pi(k+i)}$ lies in the interior of the simplex $\left[v_{0}, \ldots, v_{k}, v_{\pi(k+1)}, \ldots, v_{\pi(k+i)}\right] \subseteq S_{k+i}$ and therefore, does not belong to the proper face $\left[v_{0}, \ldots, v_{k}, v_{\pi(k+1)}, \ldots, v_{\pi(k+i-1)}\right]$ of that simplex. Since that face equals $\sigma \cap S_{k+i-1}$, it follows that $w_{0}^{0 \ldots k \pi(k+1) \ldots \pi(k+i)} \notin S_{k+i-1}$.

Note that, for $\sigma=\left[v_{0}, v_{1}\right]$ and $\tau=\left[v_{0}\right]$, the permutation $\pi:\{1\} \rightarrow\{1\}$ is the identity $\iota$ and $d_{\iota}^{01}=\left[w_{0}^{0}, w_{0}^{01}\right]$. If $\sigma=\left[v_{0}, v_{1}, v_{2}\right]$ and $\tau=\left[v_{0}, v_{1}\right]$, then $\pi:\{2\} \rightarrow\{2\}$ is the identity $\iota$ and $d_{\pi}^{012}=\left[w_{0}^{01}, w_{0}^{012}\right]$. If $\sigma=\left[v_{0}, v_{1}, v_{2}\right]$ and $\tau=\left[v_{0}\right]$, there are two permutations of $\{1,2\}$, the identity permutation $\iota$ and the permutation $\varepsilon$, which interchanges 1 and 2 . Accordingly, one obtains two 2-simplices, $d_{\iota}^{012}=\left[w_{0}^{0}, w_{0}^{01}, w_{0}^{012}\right]$ and $d_{\pi}^{012}=\left[w_{0}^{0}, w_{0}^{02}, w_{0}^{012}\right]$. formula

For $\tau=\left[v_{0}, \ldots, v_{k}\right], 0 \leq k<n$, we define the peripheral $n$-cell $c_{\pi}^{\tau}$ by the

$$
c_{\pi}^{\tau}=c^{\tau} \oplus d_{\pi}^{\tau} .
$$

Note that the intersection of $S_{k}=\operatorname{Aff}\left(c^{\tau}\right)$ and $T_{k}=\operatorname{Aff}\left(d_{\pi}^{\tau}\right)$ is the point $w_{0}^{\tau}=$ $w_{0}^{0 \ldots k}$, because the points listed in (3.8) are affinely independent. Therefore, the direct sum $c^{\tau} \oplus d_{\pi}^{\tau}$ is well-defined and has $w_{0}^{\tau}$ for its reference point. It is an $n$-dimensional convex polytope and

$$
c^{\tau} \oplus d_{\pi}^{\tau}=c^{\tau}+d_{\pi}^{\tau}-w_{0}^{\tau} .
$$

Note that of the nine peripheral 2-cells of the decomposition $L\left[v_{0}, v_{1}, v_{2}\right]$ (see Figure 2), formula (3.7) describes only three, i.e., for $\tau=\left[v_{0}\right]$, the triangles with vertices $w_{0}^{0}, w_{0}^{01}, w_{0}^{012}$ and $w_{0}^{0}, w_{0}^{02}, w_{0}^{012}$ and for $\tau=\left[v_{0}, v_{1}\right]$, the parallelogram with vertices $w_{0}^{01}, w_{1}^{01}, w_{0}^{012}, w_{1}^{012}$. To obtain the triangles with 
vertices $w_{1}^{1}, w_{1}^{12}, w_{1}^{012}$ and $w_{1}^{1}, w_{1}^{01}, w_{1}^{012}$ and the parallelogram with vertices $w_{1}^{12}, w_{2}^{12}, w_{1}^{012}, w_{2}^{012}$, we must use the faces $\tau=\left[v_{1}\right]$ and $\tau=\left[v_{1}, v_{2}\right]$, respectively. To obtain the remaining three peripheral 2-cells of $L\left[v_{0}, v_{1}, v_{2}\right]$, we must use $\tau=\left[v_{2}\right]$ and $\tau=\left[v_{0}, v_{2}\right]$. Let us illustrate, in the case of the triangle $\left[w_{1}^{1}, w_{1}^{12}, w_{1}^{012}\right]$, how it is obtained using formula (3.7) and the relabelling of the vertices of $\left[v_{0}, v_{1}, v_{2}\right]$, which sends the indices $0,1,2$ to $1,2,0$, respectively. By $(3.7), d_{\iota}^{0}=\left[w_{0}^{0}, w_{0}^{01}, w_{0}^{012}\right]$, where $\iota$ is the identity permutation on the set $\{1,2\}$. After the relabelling, to $\iota$ corresponds the identity permutation $\kappa$ on $\{0,2\}$ and we obtain $d_{\kappa}^{1}=\left[w_{1}^{1}, w_{1}^{12}, w_{1}^{012}\right]$.

The following lemma lists the vertices of $c_{\pi}^{\tau}$ and describes the faces of $c_{\pi}^{\tau}$.

LEMma 3.2. If $\sigma=\left[v_{0}, \ldots, v_{n}\right], \tau=\left[v_{0}, \ldots, v_{k}\right], 0 \leq k<n$, and $d_{\pi}^{\tau}$ is the simplex defined by (3.7), then the vertices of $c_{\pi}^{\tau}=c_{\pi}^{\tau} \oplus d_{\pi}^{\tau}$ are the points (3.11)

$$
\begin{array}{lllll}
w_{0}^{0 \ldots k} & \ldots & w_{j}^{0 \ldots k} & \ldots & w_{k}^{0 \ldots k} \\
w_{0}^{0 \ldots k \pi(k+1)} & \ldots & w_{j}^{0 \ldots k \pi(k+1)} & \ldots & w_{k}^{0 \ldots k \pi(k+1)} \\
\ldots & \ldots & \ldots & \ldots & \ldots \\
w_{0}^{0 \ldots k \pi(k+1) \ldots \pi(k+i)} & \ldots & w_{j}^{0 \ldots k \pi(k+1) \ldots \pi(k+i)} & \ldots & w_{k}^{0 \ldots k \pi(k+1) \ldots \pi(k+i)} \\
\ldots & \ldots & \ldots & \ldots & \ldots \\
w_{0}^{0 \ldots n} & \ldots & w_{j}^{0 \ldots n} & \ldots & w_{k}^{0 \ldots n} .
\end{array}
$$

The faces of $c_{\pi}^{\tau}$ are convex hulls of the sets of vertices lying at the crossings of a collection of rows with a collection of columns of (3.11). The $n$-cell $c_{\pi}^{\tau}$ is contained in $\sigma$.

Note that the last row of (3.11) consists of the points

$$
w_{j}^{0 \ldots k \pi(k+1) \pi(k+2) \ldots \pi(n)}=w_{j}^{0 \ldots n} .
$$

We will refer to (3.11) as to the vertex scheme of $c_{\pi}^{\tau}$. The crossings of a collection of rows with a collection of columns of (3.11) form a subscheme of the vertex scheme of $c_{\pi}^{\tau}$. Such a subscheme determines a face of $c_{\pi}^{\tau}$ and we call it the vertex scheme of the face.

Proof of Lemma 3.2. Since $w_{j}^{0 \ldots k}, 0 \leq j \leq k$, are the vertices of $c^{\tau}$ and $w_{0}^{0 \ldots k \pi(k+1) \ldots \pi(k+i)}, 0 \leq i \leq n-k$, are the vertices of $d_{\pi}^{\tau}$ (for $i=0$, one interprets $\pi(k+1) \ldots \pi(k+i)$ as empty), (3.9) implies that the vertices of $c_{\pi}^{\tau}$ are the points

$$
w_{j}^{0 \ldots k}+w_{0}^{0 \ldots k \pi(k+1) \ldots \pi(k+i)}-w_{0}^{0 \ldots k} .
$$

Now note that (3.2) implies

$$
w_{j}^{0 \ldots n}-w_{0}^{0 \ldots n}=\frac{1}{2}\left(v_{j}-v_{0}\right), 0 \leq j \leq n .
$$

Analogously,

$$
w_{j}^{0 \ldots k}-w_{0}^{0 \ldots k}=\frac{1}{2}\left(v_{j}-v_{0}\right)=w_{j}^{0 \ldots k \pi(k+1) \ldots \pi(k+i)}-w_{0}^{0 \ldots k \pi(k+1) \ldots \pi(k+i)}
$$


and we see that (3.12) coincides with

$$
w_{j}^{0 \ldots k \pi(k+1) \ldots \pi(k+i)} .
$$

Note that the point $w_{j}^{0 \ldots k \pi(k+1) \ldots \pi(k+i)}$ lies at the crossing of the $i$-th row and the $j$-th column of (3.11). To obtain the vertices of a face of $c_{\pi}^{\tau}$ one considers the vertices of a face of $c^{0 \ldots k}$, which are points $w_{j}^{0 \ldots k}$, for appropriate indices $0 \leq j_{1}, \ldots, j_{q} \leq k$, and one considers the vertices of a face of $d_{\pi}^{0 \ldots k}$, which are points $w_{0}^{0 \ldots k \pi(k+1) \ldots \pi(k+i)}$, for appropriate indices $0 \leq i_{1}, \ldots, i_{p} \leq n-k$. Then the points $w_{j}^{0 \ldots k}+w_{0}^{0 \ldots k \pi(k+1) \ldots \pi(k+i)}-w_{0}^{0 \ldots k}=w_{j}^{0 \ldots k \pi(k+1) \ldots \pi(k+i)}$ at the crossings of the rows determined by $i_{1}, \ldots, i_{p}$ and the columns determined by $j_{1}, \ldots, j_{q}$ are the the vertices of the given face of $c_{\pi}^{\tau}$. Finally, since every convex polytope is the convex hull of its vertices and the points in (3.11) belong to $\sigma$, one concludes that $c_{\pi}^{\tau} \subseteq \sigma$.

REMARK 3.3. In formula (3.7) the index 0 plays a special role and the notation for the $(n-k)$-simplex $d_{\pi}^{\tau}$ should really include that index, i.e., one should be using the notation, say $d_{0 \pi}^{\tau}$. Then (3.10) should be written as $c^{\tau} \oplus d_{0 \pi}^{\tau}=c^{\tau}+d_{0 \pi}^{\tau}-w_{0}^{0 \ldots k}$. Choosing another index $j \in\{0, \ldots, k\}$ would yield another $(n-k)$-simplex $d_{j \pi}^{\tau}=\left[w_{j}^{0 \ldots k}, w_{j}^{0 \ldots k \pi(k+1)}, \ldots, w_{j}^{0 \ldots n}\right]$ and the analogue of (3.10) should be written as $c^{\tau} \oplus d_{j \pi}^{\tau}=c^{\tau}+d_{j \pi}^{\tau}-w_{j}^{0 \ldots k}$. The fact that (3.12) and (3.15) coincide show that $d_{j \pi}^{\tau}=d_{0 \pi}^{\tau}+\left(w_{j}^{0 \ldots k}-w_{0}^{0 \ldots k}\right)$ and thus, $d_{j \pi}^{\tau}$ is the translate of $d_{0 \pi}^{\tau}$ by the vector $w_{j}^{0 \ldots k}-w_{0}^{0 \ldots k}$, which connects two points of Aff $\left(c^{\tau}\right)$. Therefore, Aff $\left(c^{\tau}\right)$ and Aff $\left(d_{j \pi}^{\tau}\right)$ intersect in the single point $w_{0}^{0 \ldots k}+\left(w_{j}^{0 \ldots k}-w_{0}^{0 \ldots k}\right)=w_{j}^{0 \ldots k}$, which proves that the direct sum $c^{\tau} \oplus d_{j \pi}^{\tau}$ is well defined and has $w_{j}^{0 \ldots k}$ for its reference point. Moreover, $c^{\tau} \oplus d_{j \pi}^{\tau}=c^{\tau}+d_{j \pi}^{\tau}-w_{j}^{0 \ldots k}=c^{\tau}+d_{0 \pi}^{\tau}-w_{0}^{0 \ldots k}=c^{\tau} \oplus d_{0 \pi}^{\tau}$. This shows that the choice of the index $j$ does not affect the definition of the $n$-cell $c_{\pi}^{\tau}$ and therefore, there is no need for including that index in the notation for $c_{\pi}^{\tau}$. Note that the vertices of $c^{\tau}$ form the first row of the vertex scheme (3.11) and the vertices of $d_{j \pi}^{\tau}$ form the $j$-th column of that scheme. Their crossing $w_{j}^{0 \ldots k}$ is the reference point of $c^{\tau} \oplus d_{j \pi}^{\tau}$. To simplify notation we will generally continue to denote $d_{0 \pi}^{\tau}$ by $d_{\pi}^{\tau}$.

3.4. Every vertex $w_{t}^{r \ldots s}$ of $c_{\pi}^{\tau}$ (in fact, every point of $\sigma$ ) belongs to the interior of a unique face of $\sigma$. We refer to the dimension of that face as to the rank of the vertex $w_{t}^{r \ldots s}$. By $(3.2), w_{t}^{r \ldots s}=\frac{1}{2}\left(v_{t}+b^{r \ldots s}\right)$ lies in the interior of the face $\left[v_{r}, \ldots, v_{s}\right]$ of $\sigma$ and thus, the rank of $w_{t}^{r \ldots s}$ equals $\operatorname{dim}\left[v_{r}, \ldots, v_{s}\right]=$ $\operatorname{card}\{r, \ldots, s\}-1$. Note that the vertices $w_{j}^{0 \ldots k}$ in the 0 -th row of $(3.11)$ are of rank $k$ and the vertices $w_{j}^{0 \ldots k \pi(k+1) \ldots \pi(k+i)}$ in the $i$-th row of $(3.11)$ are of rank $k+i$. Clearly, the first summand $c^{\tau}$ of $c^{\tau} \oplus d_{\pi}^{\tau}=c^{\tau} \oplus d_{j \pi}^{\tau}$ is the simplex spanned by the vertices of $c_{\pi}^{\tau}$ of minimal rank and is therefore, completely determined by $c_{\pi}^{\tau}$. The vertices of the second summand $d_{j \pi}^{\tau}$ have 
a natural linear order given by their ranks, which increase by 1 as we descend along the $j$-th column. Since (3.12) and (3.15) coincide, the translation by $w_{j}^{\tau}-w_{0}^{\tau}$ is an affine isomorphism $d_{\pi}^{\tau}=d_{0 \pi}^{\tau} \rightarrow d_{j \pi}^{\tau}$ which preserves the ranks, because it sends the vertex $w_{0}^{0 \ldots k \pi(k+1) \ldots \pi(k+i)}$ of rank $k+i$ to the vertex $w_{j}^{0 \ldots k \pi(k+1) \ldots \pi(k+i)}$, whose rank is also $k+i$. Consequently, the translation by $w_{j}^{\tau}-w_{0}^{\tau}$ preserves the ordering of the vertices of $d_{\pi}^{\tau}=d_{0 \pi}^{\tau}$ and $d_{j \pi}^{\tau}$.

If $a$ is a face of an $n$-cell $c_{\pi}^{\tau}=c^{\tau} \oplus d_{\pi}^{\tau} \in L(\sigma)$, one cannot conclude that $a=c_{a} \oplus d_{a}$, where $c_{a} \leq c^{\tau}, d_{a} \leq d_{\pi}^{\tau}$, because Aff $\left(c_{a}\right) \cap$ Aff $\left(d_{a}\right)$ could be empty and the direct sum $c_{a} \oplus d_{a}$ would not be defined. However, the following lemma holds.

LEMmA 3.4. Let $c=c^{\tau} \oplus d_{\pi}^{\tau}$ be an $n$-cell from $L(\sigma)$ and let $a \leq c$ be a face of $c$, whose vertex scheme is the following subscheme of the vertex scheme (3.11) of $c$.

$$
\begin{array}{llll}
w_{j}^{0 \ldots k \pi(k+1) \ldots \pi(k+i)} & w_{j_{1}}^{0 \ldots k \pi(k+1) \ldots \pi(k+i)} & \ldots & w_{j_{s}}^{0 \ldots k \pi(k+1) \ldots \pi(k+i)} \\
w_{j}^{0 \ldots k \pi(k+1) \ldots \pi\left(k+i_{1}\right)} & w_{j_{1}}^{0 \ldots k \pi(k+1) \ldots \pi\left(k+i_{1}\right)} & \ldots & w_{j_{s}}^{0 \ldots k \pi(k+1) \ldots \pi\left(k+i_{1}\right)} \\
\ldots & \ldots & \ldots & \\
w_{j}^{0 \ldots k \pi(k+1) \ldots \pi\left(k+i_{r}\right)} & w_{j_{1}}^{0 \ldots k \pi(k+1) \ldots \pi\left(k+i_{r}\right)} & \ldots & w_{j_{s}}^{0 \ldots k \pi(k+1) \ldots \pi\left(k+i_{r}\right)},
\end{array}
$$

where $0 \leq i=i_{0} \leq i_{1}<\ldots<i_{r} \leq n-k$ and $0 \leq j=j_{0} \leq j_{1}<\ldots<j_{s} \leq k$. If $c_{a}$ and $d_{a}$ are simplices, whose vertex schemes are the first row and the first column of (3.16), respectively, then $c_{a}$ and $d_{a}$ are faces of a such that the direct sum decomposition $a=c_{a} \oplus d_{a}=c_{a}+d_{a}-w$ is well defined and the corresponding reference point $w=w_{j}^{0 \ldots k \pi(k+1) \ldots \pi(k+i)}$, lying at the crossing of the first row with the first column of (3.16), is a vertex of both simplices $c_{a}$ and $d_{a}$. The set of vertices of $c_{a}$ coincides with the set of vertices of the face a having minimal rank. Therefore, $c_{a}$ is completely determined by a and does not depend on $c$. The simplex $c^{\prime}{ }_{a}=\left[w_{j}^{0 \ldots k}, w_{j_{1}}^{0 \ldots k}, \ldots, w_{j_{s}}^{0 \ldots k}\right]$ is a face of $c^{\tau}$ and translation by $w_{0}^{0 \ldots k \pi(k+1) \ldots \pi(k+i)}-w_{0}^{\tau}$ is a bijection between the vertices of $c^{\prime}{ }_{a}$ and $c_{a}$ and thus, $c_{a}=c^{\prime}{ }_{a}+w_{0}^{0 \ldots k \pi(k+1) \ldots \pi(k+i)}-w_{0}^{\tau}$. The simplex $d^{\prime}{ }_{a}=\left[w_{0}^{0 \ldots k \pi(k+1) \ldots \pi(k+i)}, w_{0}^{0 \ldots k \pi(k+1) \ldots \pi\left(k+i_{1}\right)}, \ldots, w_{0}^{0 \ldots k \pi(k+1) \ldots \pi\left(k+i_{r}\right)}\right]$ is a face of $d_{\pi}^{\tau}$ and $a=c^{\prime}{ }_{a}+d^{\prime}{ }_{a}-w_{0}^{\tau}$. Translation by $w_{j}^{\tau}-w_{0}^{\tau}$ is a bijection between the vertices of $d^{\prime}{ }_{a}$ and $d_{a}$ and thus, $d_{a}=d^{\prime}{ }_{a}+w_{j}^{\tau}-w_{0}^{\tau}$. This translation preserves the ranks of the vertices of $d^{\prime}{ }_{a}$ and thus, it is an order preserving isomorphism between the simplices $d^{\prime}{ }_{a}$ and $d_{a}$. Analogously, if $d_{a}^{1}$ is the face of a, whose vertex scheme is the l-th column of (3.16), then translation by $w_{j_{l}}^{\tau}-w_{j}^{\tau}$ is an order preserving isomorphism between the simplices $d_{a}$ and $d_{a}^{1}$.

Proof. All the assertions of this lemma are immediate consequences of the definitions and facts given in Subsections 2.7, 3.1, 3.2 and 3.3. They are here stated only for easier referencing. The vertices of $c_{a}$ lie 
in the first row of (3.16) and have rank $k+i$, while all other vertices of $a$ have ranks $>k+i$. Therefore, $c_{a}$ is completely determined by $a$. The vertex scheme $w_{j}^{0 \ldots k} w_{j_{1}}^{0 \ldots k} \ldots w_{j_{s}}^{0 \ldots k}$ of $c^{\prime}{ }_{a}$ is a subscheme of the vertex scheme $w_{0}^{\tau} \ldots w_{k}^{\tau}$ of $c^{\tau}$ and thus, $c^{\prime}{ }_{a} \leq c^{\tau}$. Since $w_{0}^{0 \ldots k \pi(k+1) \ldots \pi(k+i)}-$ $w_{0}^{\tau}=w_{j_{l}}^{0 \ldots k \pi(k+1) \ldots \pi(k+i)}-w_{j_{l}}^{\tau}$, translation by $w_{0}^{0 \ldots k \pi(k+1) \ldots \pi(k+i)}-w_{0}^{\tau}$ maps the vertex $w_{j_{l}}^{0 \ldots k}$ of $c^{\prime}{ }_{a}$ to the vertex $w_{j_{l}}^{0 \ldots k \pi(k+1) \ldots \pi(k+i)}$ of $c_{a}$ and thus, $c_{a}=c^{\prime}{ }_{a}+w_{0}^{0 \ldots k \pi(k+1) \ldots \pi(k+i)}-w_{0}^{\tau}$. Analogously, the vertex scheme of $d^{\prime}{ }_{a}$ is a subscheme of the vertex scheme of $d_{\pi}^{\tau}$ and thus, $d^{\prime}{ }_{a} \leq d_{\pi}^{\tau}$. Since $w_{j}^{\tau}-w_{0}^{\tau}=w_{j}^{0 \ldots k \pi(k+1) \ldots \pi\left(k+i_{t}\right)}-w_{0}^{0 \ldots k \pi(k+1) \ldots \pi\left(k+i_{t}\right)}$, translation by $w_{j}^{\tau}-w_{0}^{\tau}$ maps the vertex $w_{0}^{0 \ldots k \pi(k+1) \ldots \pi\left(k+i_{t}\right)}$ of $d^{\prime}{ }_{a}$ to the vertex $w_{j}^{0 \ldots k \pi(k+1) \ldots \pi\left(k+i_{t}\right)}$ of $d_{a}$ and thus, $d_{a}=d^{\prime}{ }_{a}+w_{j}^{\tau}-w_{0}^{\tau}$. Both vertices $w_{0}^{0 \ldots k \pi(k+1) \ldots \pi\left(k+i_{t}\right)}$ and $w_{j}^{0 \ldots k \pi(k+1) \ldots \pi\left(k+i_{t}\right)}$ have the same rank $k+i_{t}$, which shows that this translation is an order preserving isomorphism between the simplices $d^{\prime}{ }_{a}$ and $d_{a}$. A similar argument proves the assertion concerning the translation by $w_{j_{l}}^{\tau}-w_{j}^{\tau}$.

REMARK 3.5. If $\sigma=\left[v_{0}, \ldots, v_{n}\right] \in K$, there exists a bijection between the set of $n$-cells of $L(\sigma)$ and the elements of the partially ordered set $C[n]$, which consists of chains of nonempty subsets of the set $\{0, \ldots, n\}$, ordered by proper inclusion $\subset$ and having two additional properties. The adjacent links of a chain are sets whose cardinality differs by 1 and the terminal link is the subset $\{0, \ldots, n\}$. In that sense $L(\sigma)$ can be considered a geometric representation of $C[n]$. E.g., $C[2]$ consists of ten chains ending with $\{0,1,2\}$. They are the 1-link chain: $\{0,1,2\}$, three 2-link chains: $\{0,1\} \subset\{0,1,2\},\{0,2\} \subset$ $\{0,1,2\},\{1,2\} \subset\{0,1,2\}$ and six 3-link chains: $\{0\} \subset\{0,1\} \subset\{0,1,2\}$, $\{0\} \subset\{0,2\} \subset\{0,1,2\},\{1\} \subset\{0,1\} \subset\{0,1,2\},\{1\} \subset\{1,2\} \subset\{0,1,2\}$, $\{2\} \subset\{0,2\} \subset\{0,1,2\},\{2\} \subset\{1,2\} \subset\{0,1,2\}$.

3.5. The main result in this section is Theorem 3.6. Its lengthy proof is carried out in a number of steps.

Theorem 3.6. For any $n$-simplex $\sigma$ of a simplicial complex $K$, the $n$ cells $c_{\pi}^{\tau}$ described in Subsections 3.2 and 3.3, together with their faces, form a cellular subdivision $L(\sigma)$ of $\sigma$. If $\zeta<\sigma$ is a proper face of $\sigma$, then $L(\zeta) \subseteq L(\sigma)$ and

$$
L(\zeta)=\zeta \cap L(\sigma)=\{\zeta \cap c \mid c \in L(\sigma)\} .
$$

The union $L(K)=\bigcup_{\sigma \in K} L(\sigma)$ is a cellular subdivision of $K$.

We will first prove that $L(\sigma)$ is a cellular complex. For this we need to prove that the intersection of any two cells $c_{1}, c_{2} \in L(\sigma)$ is a common face (possibly empty) of these cells and that the union of all cells $c \in L(\sigma)$ equals $\sigma$. If $\operatorname{dim} \sigma=n$, it suffices to prove the first assertion in the special case when 
$c_{1}, c_{2}$ are $n$-dimensional cells of $L(\sigma)$. Indeed, if $c_{1}, c_{2}$ are arbitrary cells from $L(\sigma)$, then there exist $n$-dimensional cells $c_{1}^{\prime}, c_{2}^{\prime} \in L(\sigma)$ such that $c_{1} \leq c_{1}^{\prime}$ and $c_{2} \leq c_{2}^{\prime}$. By the assertion in the special case, the intersection $c_{1}^{\prime} \cap c_{2}^{\prime}$ is a cell $c^{\prime}$, which is a common face of $c_{1}^{\prime}$ and $c_{2}^{\prime}$. Since $c_{1}=c_{1} \cap c_{1}^{\prime}$ and $c_{2}=c_{2} \cap c_{2}^{\prime}$, we see that $c_{1} \cap c_{2}=c_{1} \cap\left(c_{1}^{\prime} \cap c_{2}^{\prime}\right) \cap c_{2}=c_{1} \cap c^{\prime} \cap c_{2}$. The cells $c_{1}, c^{\prime}$ are faces of $c_{1}^{\prime}$ and thus, their intersection $c_{1} \cap c^{\prime}$ is also a face of $c^{\prime}$. Analogously, $c_{2} \cap c^{\prime}$ is a face of $c^{\prime}$. Consequently, the intersection $c_{1} \cap c_{2}=\left(c_{1} \cap c^{\prime}\right) \cap\left(c^{\prime} \cap c_{2}\right)$ is a face of $c^{\prime}$. Since $c^{\prime} \leq c_{1}^{\prime} \in L(\sigma)$, it follows that $c^{\prime} \in L(\sigma)$ and thus, also $c_{1} \cap c_{2} \in L(\sigma)$. Clearly, to prove the second assertion, it suffices to prove that $\sigma$ is the union of all $n$-cells from $L(\sigma)$.

3.6. Before considering the intersection $c_{\pi}^{\tau} \cap c_{\pi^{\prime}}^{\tau^{\prime}}$ of two different $n$-cells from $L(\sigma)$, we will prove the following lemma concerning the intersection $d_{\pi}^{\tau} \cap d_{\pi^{\prime}}^{\tau}$

LeMma 3.7. Let $\sigma=\left[v_{0}, \ldots, v_{n}\right]$, let $\tau=\left[v_{0}, \ldots, v_{k}\right], 0 \leq k<n$, and let $\pi, \pi^{\prime}$ be permutations of the set $\{k+1, \ldots, n\}$. Then $d_{\pi}^{\tau} \cap d_{\pi^{\prime}}^{\tau}$ is a common face of both simplices $d_{\pi}^{\tau}$ and $d_{\pi^{\prime}}^{\tau}$. More precisely, if $r_{1}<r_{2}<\ldots<r_{p}=n-k$ are all integers in the set $\{1, \ldots, n-k\}$, for which

$$
\pi\left\{k+1, \ldots, k+r_{i}\right\}=\pi^{\prime}\left\{k+1, \ldots, k+r_{i}\right\},
$$

then

$$
d_{\pi}^{\tau} \cap d_{\pi^{\prime}}^{\tau}=d_{\pi \pi^{\prime}}^{\tau}
$$

where

$$
d_{\pi \pi^{\prime}}^{\tau}=\left[w_{0}^{0 \ldots k}, w_{0}^{0 \ldots k \pi(k+1) \ldots \pi\left(k+r_{1}\right)}, w_{0}^{0 \ldots k \pi(k+1) \ldots \pi\left(k+r_{1}\right) \ldots \pi\left(k+r_{2}\right)}, \ldots, w_{0}^{0 \ldots n}\right] .
$$

ProOF. First note that the vertices of $d_{\pi \pi^{\prime}}^{\tau}$ are contained in $d_{\pi}^{\tau} \cap d_{\pi^{\prime}}^{\tau}$, because by (3.18), $\left\{\pi(k+1), \ldots, \pi\left(k+r_{i}\right)\right\} \stackrel{=}{=}\left\{\pi^{\prime}(k+1), \ldots, \pi^{\prime}\left(k+r_{i}\right)\right\}$. Since $d_{\pi}^{\tau} \cap d_{\pi^{\prime}}^{\tau}$ is convex, it follows that

$$
d_{\pi \pi^{\prime}}^{\tau} \subseteq d_{\pi}^{0 \ldots k} \cap d_{\pi^{\prime}}^{0 \ldots k}
$$

To prove the opposite inclusion, consider the $n$-dimensional affine manifold $S_{n}=\operatorname{Aff}\left\{v_{0}, \ldots, v_{n}\right\}$. Moreover, for any pair of integers $j, l \in$ $\{1, \ldots, n-k\}$, consider the $(n-1)$-dimensional affine manifold $S_{j l} \subseteq S_{n}$, spanned by the points $v_{i}, i \in\{0, \ldots, n\} \backslash\{\pi(k+j), \pi(k+l)\}$ and by the point $\frac{1}{2}\left(v_{\pi(k+j)}+v_{\pi(k+l)}\right)$. It consists of all points $u \in S_{n}$, whose barycentric coordinates satisfy the condition $\lambda_{\pi(k+j)}=\lambda_{\pi(k+l)}$. Since $\pi(k+j), \pi(k+l)>k$, we read from (3.2) that

$$
\lambda_{\pi(k+j)}\left(w_{0}^{0 \ldots k}\right)=0=\lambda_{\pi(k+l)}\left(w_{0}^{0 \ldots k}\right),
$$

and thus, $w_{0}^{0 \ldots k} \in S_{j l}$. Since $w_{0}^{0 \ldots k} \in d_{\pi}^{\tau} \cap d_{\pi^{\prime}}^{\tau}$, we see that

$$
\left\{w_{0}^{0 \ldots k}\right\} \subseteq d_{\pi}^{\tau} \cap d_{\pi^{\prime}}^{\tau} \cap S_{j l}
$$


and thus,

$$
d_{\pi}^{\tau} \cap S_{j l} \neq \emptyset, d_{\pi^{\prime}}^{\tau} \cap S_{j l} \neq \emptyset .
$$

For every integer $j \in\{1, \ldots, n-k\}$ there is a unique integer $j^{\prime} \in$ $\{1, \ldots, n-k\}$ such that $\pi(k+j)=\pi^{\prime}\left(k+j^{\prime}\right)$. We will say that an ordered pair of integers $(j, l)$ from $\{1, \ldots, n-k\}$ is an inversion pair provided $j<l$ and $j^{\prime}>l^{\prime}$. Let us show that, for all inversion pairs $(j, l), S_{j l}$ is a supporting hyperplane for both simplices $d_{\pi}^{\tau}, d_{\pi^{\prime}}^{\tau}$ and it separates them. More precisely, $S_{j l}$ determines in $S_{n}$ two affine halfspaces,

$$
\begin{aligned}
& S_{j l}^{+}=\left\{u \in S_{n} \mid \lambda_{\pi(k+j)}(u) \geq \lambda_{\pi(k+l)}(u)\right\}, \\
& S_{j l}^{-}=\left\{u \in S_{n} \mid \lambda_{\pi(k+j)}(u) \leq \lambda_{\pi(k+l)}(u)\right\},
\end{aligned}
$$

such that

$$
\begin{aligned}
& d_{\pi}^{\tau} \subseteq S_{j l}^{+} \\
& d_{\pi^{\prime}}^{\tau} \subseteq S_{j l}^{-}
\end{aligned}
$$

and (3.24) holds.

To prove (3.27) and (3.28), it suffices to prove that, for $1 \leq i \leq n-k$, all the vertices $w_{0}^{0 \ldots k \pi(k+1) \ldots \pi(k+i)}$ of $d_{\pi}^{0 \ldots k}$ satisfy the inequality

$$
\lambda_{\pi(k+j)}\left(w_{0}^{0 \ldots k \pi(k+1) \ldots \pi(k+i)}\right) \geq \lambda_{\pi(k+l)}\left(w_{0}^{0 \ldots k \pi(k+1) \ldots \pi(k+i)}\right)
$$

and all the vertices $w_{0}^{0 \ldots k \pi^{\prime}(k+1) \ldots \pi^{\prime}(k+i)}$ of $d_{\pi^{\prime}}^{0 \ldots k}$ satisfy the inequality

$$
\lambda_{\pi(k+j)}\left(w_{0}^{0 \ldots k \pi^{\prime}(k+1) \ldots \pi^{\prime}(k+i)}\right) \leq \lambda_{\pi(k+l)}\left(w_{0}^{0 \ldots k \pi^{\prime}(k+1) \ldots \pi^{\prime}(k+i)}\right) .
$$

Indeed, by (3.2), applied to the simplex $\left[v_{0} \ldots, v_{k}, v_{\pi(k+1)} \ldots, v_{\pi(k+i)}\right]$,

$$
\begin{aligned}
& w_{0}^{0 \ldots k \pi(k+1) \ldots \pi(k+i)}= \\
& \frac{1}{2} v_{0}+\frac{1}{2(k+i+1)}\left(v_{0}+\ldots+v_{k}+v_{\pi(k+1)}+\ldots+v_{\pi(k+i)}\right)
\end{aligned}
$$

and thus,

$$
\lambda_{\pi(k+j)}\left(w_{0}^{0 \ldots k \pi(k+1) \ldots \pi(k+i)}\right)= \begin{cases}\frac{1}{2(k+i+1)}, & j \leq i, \\ 0, & j>i .\end{cases}
$$

Replacing $j$ by $l$, an analogous relation for $\lambda_{\pi(k+l)}\left(w_{0}^{0 \ldots k \pi(k+1) \ldots \pi(k+i)}\right)$ is obtained. Using temporarily the abbreviation $w$ for $w_{0}^{0 \ldots k \pi(k+1) \ldots \pi(k+i)}$, we see that the two relations yield the following.

$$
\begin{gathered}
\lambda_{\pi(k+j)}(w)=0=\lambda_{\pi(k+l)}(w), i<j, \\
\lambda_{\pi(k+j)}(w)=\frac{1}{2(k+i+1)}>0=\lambda_{\pi(k+l)}(w), j \leq i<l, \\
\lambda_{\pi(k+j)}(w)=\frac{1}{2(k+i+1)}=\lambda_{k+l}(w), l \leq i .
\end{gathered}
$$

Clearly, formulae (3.33), (3.34) and (3.35) imply (3.29). 
For $\lambda_{\pi(k+j)}\left(w_{0}^{0 \ldots k \pi^{\prime}(k+1) \ldots \pi^{\prime}(k+i)}\right)$ we have a relation analogous to $(3.31)$.

$$
\begin{aligned}
& w_{0}^{0 \ldots k \pi^{\prime}(k+1) \ldots \pi^{\prime}(k+i)}= \\
& \frac{1}{2} v_{0}+\frac{1}{2(k+i+1)}\left(v_{0}+\ldots+v_{k}+v_{\pi^{\prime}(k+1)}+\ldots+v_{\pi^{\prime}(k+i)}\right) .
\end{aligned}
$$

Since $\pi(k+j)=\pi^{\prime}\left(k+j^{\prime}\right)$, we see that

$$
\lambda_{\pi(k+j)}\left(w_{0}^{0 \ldots k \pi^{\prime}(k+1) \ldots \pi^{\prime}(k+i)}\right)= \begin{cases}\frac{1}{2(k+i+1)}, & j^{\prime} \leq i, \\ 0, & j^{\prime}>i .\end{cases}
$$

Replacing $j$ by $l$, an analogous relation for $\lambda_{\pi(k+l)}\left(w_{0}^{0 \ldots k \pi^{\prime}(k+1) \ldots \pi^{\prime}(k+i)}\right)$ is obtained. Using temporarily the abbreviation $w^{\prime}$ for $w_{0}^{0 \ldots k \pi^{\prime}(k+1) \ldots \pi^{\prime}(k+i)}$, we see that the two relations yield the following.

$$
\begin{gathered}
\lambda_{\pi(k+j)}\left(w^{\prime}\right)=0=\lambda_{\pi(k+l)}\left(w^{\prime}\right), i<l^{\prime}, \\
\lambda_{\pi(k+j)}\left(w^{\prime}\right)=0<\frac{1}{2(k+i+1)}=\lambda_{\pi(k+l)}\left(w^{\prime}\right), l^{\prime} \leq i<j^{\prime}, \\
\lambda_{\pi(k+j)}\left(w^{\prime}\right)=\frac{1}{2(k+i+1)}=\lambda_{\pi(k+l)}\left(w^{\prime}\right), j^{\prime} \leq i .
\end{gathered}
$$

Clearly, formulae (3.38), (3.39) and (3.40) imply (3.30).

It is a consequence of $(3.27),(3.28)$ and $S_{j l}^{+} \cap S_{j l}^{-}=S_{j l}$ that $d_{\pi}^{0 \ldots k} \cap d_{\pi^{\prime}}^{0 \ldots k} \subseteq$ $S_{j l}$ and thus,

$$
d_{\pi}^{\tau} \cap d_{\pi^{\prime}}^{\tau} \subseteq d_{\pi}^{\tau} \cap S_{j l}
$$

Denote by $W_{j l}$ the set of all vertices $w_{0}^{0 \ldots k \pi(k+1) \ldots \pi(k+i)}$ of $d_{\pi}^{\tau}$, which lie in $S_{j l}$. Since $S_{j l}$ is a supporting hyperplane of the simplex $d_{\pi}^{\tau}$ in $S_{n}$, the intersection $d_{\pi}^{\tau} \cap S_{j l}$ is the face of $d_{\pi}^{\tau}$, spanned by the set $W_{j l}$, i.e., $d_{\pi}^{\tau} \cap S_{j l}$ is the convex hull $\operatorname{Conv}\left(W_{j l}\right)$. If $\left(j_{1}, l_{1}\right), \ldots,\left(j_{m}, l_{m}\right)$ are all inversion pairs, then $\left(d_{\pi}^{\tau} \cap\right.$ $\left.S_{j_{1} l_{1}}\right) \cap \ldots \cap\left(d_{\pi}^{\tau} \cap S_{j_{m} l_{m}}\right)=\operatorname{Conv}\left(W_{j_{1} l_{1}}\right) \cap \ldots \cap \operatorname{Conv}\left(W_{j_{m} l_{m}}\right)$. However, the intersection of a collection of faces of a simplex coincides with the convex hull of the intersection of their sets of vertices. Consequently, (3.41) implies

$$
d_{\pi}^{\tau} \cap d_{\pi^{\prime}}^{\tau} \subseteq \operatorname{Conv}\left(W_{j_{1} l_{1}} \cap \ldots \cap W_{j_{m} l_{m}}\right) .
$$

To complete the proof, it suffices to show that, for every $i \in\{1, \ldots, n-1\} \backslash$ $\left\{r_{1}, \ldots, r_{p-1}\right\}$, there exists an inversion pair $(j, l)$ such that $w_{0}^{0 \ldots k \pi(k+1) \ldots \pi(k+i)}$ $\notin W_{j l}$. Indeed, if this is the case, then $W_{j_{1} l_{1}} \cap \ldots \cap W_{j_{m} l_{m}}$ is contained in the set $\left\{w_{0}^{0 \ldots k}, w_{0}^{0 \ldots k \pi(k+1) \ldots \pi\left(k+r_{1}\right)}, w_{0}^{0 \ldots k \pi(k+1) \ldots \pi\left(k+r_{2}\right)}, \ldots, w_{0}^{0 \ldots n}\right\}$, which spans the simplex $d_{\pi \pi^{\prime}}^{\tau}$. Therefore, by (3.42),

$$
d_{\pi}^{\tau} \cap d_{\pi^{\prime}}^{\tau} \subseteq d_{\pi \pi^{\prime}}^{\tau}
$$

which together with (3.21) yields the desired equality (3.19).

Finally, let us show that, for $0<i<n-k$ and $i \neq r_{1}, \ldots, r_{p}$, there does exist an inversion pair $(j, l)$ such that

$$
j \leq i<l
$$


and therefore, by (3.44), $w=w^{0 \ldots k \pi(k+1) \ldots \pi(k+i)} \notin S_{j l}$, i.e., $w \notin W_{j l}$.

First consider the case, when $0<i<r_{1}$. By the definition of $r_{1}$,

$$
\pi\{k+1, \ldots, k+i\} \neq \pi^{\prime}\{k+1, \ldots, k+i\} .
$$

We claim that there exists an integer $j \in\{1, \ldots, i\}$ such that $\pi(k+j) \notin$ $\pi^{\prime}\{k+1, \ldots, k+i\}$. Indeed, if this were not the case, we would have $\pi\{k+$ $1, \ldots, k+i\} \subseteq \pi^{\prime}\{k+1, \ldots, k+i\}$. Since these are equipotent finite sets, we would have $\pi\{k+1, \ldots, k+i\}=\pi^{\prime}\{k+1, \ldots, k+i\}$, which is in contradiction with (3.45). Since $\pi(k+j)=\pi^{\prime}\left(k+j^{\prime}\right)$, one must have $j^{\prime}>i$. Similarly, there exists an $l \in\{i+1, \ldots, n-k\}$ such that $\pi(k+l) \notin \pi^{\prime}\{k+i+1, \ldots, n\}$. If this were not the case, we would have $\pi\{k+i+1, \ldots, n\} \subseteq \pi^{\prime}\{k+i+1, \ldots, n\}$, hence also $\pi\{k+i+1, \ldots, n\}=\pi^{\prime}\{k+i+1, \ldots, n\}$. However, this too is in contradiction with (3.45), because it implies the equality $\pi\{k+1, \ldots, k+i\}=$ $\pi^{\prime}\{k+1, \ldots, k+i\}$. Since $\pi(k+l)=\pi^{\prime}\left(k+l^{\prime}\right)$, one must have $l^{\prime} \leq i$. Now $j \leq i<l$ and $l^{\prime} \leq i<j^{\prime}$ show that $(j, l)$ is an inversion pair with property $(3.44)$.

If $r_{1}<i<r_{2}$, one repeats the argument with obvious changes. Instead of $\{0, \ldots, k\}$, one considers $\left\{0, \ldots, k, \pi(k+1), \ldots, \pi\left(k+r_{1}\right)\right\}=\left\{0, \ldots, k, \pi^{\prime}(k+\right.$ $\left.1), \ldots, \pi^{\prime}\left(k+r_{1}\right)\right\}$ and one notes that $\pi$ and $\pi^{\prime}$ permute the set $\left\{k+r_{1}+\right.$ $\left.1, \ldots, k+r_{2}\right\}$. Instead of (3.45), one uses

$$
\pi\left\{k+r_{1}+1, \ldots, k+i\right\} \neq \pi^{\prime}\left\{k+r_{1}+1, \ldots, k+i\right\},
$$

which holds, because of the definition of $r_{2}$. One proceeds in the same way, when $r_{m}<i<r_{m+1}, m \geq 2$.

The next lemma is an immediate consequence of Lemma 3.7.

Lemma 3.8. Let $\sigma=\left[v_{0}, \ldots, v_{n}\right], \tau=\left[v_{0}, \ldots, v_{k}\right], 0 \leq k<n$, and let $\pi, \pi^{\prime}$ be permutations of the set $\{k+1, \ldots, n\}$. Let $W\left(d_{\pi}^{\tau}\right)$ and $W\left(d_{\pi^{\prime}}^{\tau}\right)$ be the sets of vertices of the simplices $d_{\pi}^{\tau}$ and $d_{\pi^{\prime}}^{\tau}$, respectively. Then $d_{\pi}^{\tau} \cap d_{\pi^{\prime}}^{\tau}$ is the convex hull of the set $W\left(d_{\pi}^{\tau}\right) \cap W\left(d_{\pi^{\prime}}^{\tau}\right)$.

Proof. Since $W\left(d_{\pi}^{\tau}\right) \subseteq d_{\pi}^{\tau}, W\left(d_{\pi^{\prime}}^{\tau}\right) \subseteq d_{\pi^{\prime}}^{\tau}$ and $d_{\pi}^{\tau} \cap d_{\pi^{\prime}}^{\tau}$ is convex, it follows that $\operatorname{Conv}\left(W\left(d_{\pi}^{\tau}\right) \cap W\left(d_{\pi^{\prime}}^{\tau}\right)\right) \subseteq d_{\pi}^{\tau} \cap d_{\pi^{\prime}}^{\tau}$. Conversely, by Lemma 3.7, $d_{\pi}^{\tau} \cap d_{\pi^{\prime}}^{\tau} \leq d_{\pi}^{\tau}$ and $d_{\pi}^{\tau} \cap d_{\pi^{\prime}}^{\tau} \leq d_{\pi^{\prime}}^{\tau}$. Therefore, if $w$ is a vertex of $d_{\pi}^{\tau} \cap d_{\pi^{\prime}}^{\tau}$, it is also a vertex of $d_{\pi}^{\tau}$ and a vertex of $d_{\pi^{\prime}}^{\tau}$. Consequently, $w \in W\left(d_{\pi}^{\tau}\right) \cap W\left(d_{\pi^{\prime}}^{\tau}\right) \subseteq$ Conv $\left(W\left(d_{\pi}^{\tau}\right) \cap W\left(d_{\pi^{\prime}}^{\tau}\right)\right)$. Since $d_{\pi}^{\tau} \cap d_{\pi^{\prime}}^{\tau}$ is the convex hull of the set of all of its vertices $w$, it follows that $d_{\pi}^{\tau} \cap d_{\pi^{\prime}}^{\tau} \subseteq \operatorname{Conv}\left(W\left(d_{\pi}^{\tau}\right) \cap W\left(d_{\pi^{\prime}}^{\tau}\right)\right)$.

3.7. In considering the intersection of two different $n$-cells $c_{\pi^{\prime}}^{\tau^{\prime}}, c_{\pi^{\prime \prime}}^{\tau^{\prime \prime}} \in$ $L(\sigma)$, we distinguish three cases: $(a) \tau^{\prime}=\tau^{\prime \prime},(b) \tau^{\prime} \neq \tau^{\prime \prime}$ and the convex hull $\bar{\tau}=\operatorname{Conv}\left(\tau^{\prime} \cup \tau^{\prime \prime}\right)=\sigma$ and $(c) \tau^{\prime} \neq \tau^{\prime \prime}$ and $\bar{\tau}$ is a proper face of $\sigma$. The assertion that $c_{\pi^{\prime}}^{\tau^{\prime}} \cap c_{\pi^{\prime \prime}}^{\tau^{\prime \prime}}$ is a common face of the cells $c_{\pi^{\prime}}^{\tau^{\prime}}$ and $c_{\pi^{\prime \prime}}^{\tau^{\prime \prime}}$ in cases $(a)$, $(b)$ and $(c)$ will be proved in Lemmas $3.9,3.10$ and 3.12, respectively. 
3.7.1. Case $(a), \tau^{\prime}=\tau^{\prime \prime}$. Clearly, $\tau^{\prime} \neq \sigma$, because there is only one central $n$-cell $c^{\sigma}$. By relabelling the vertices of $\sigma$, it suffices to consider the case of a proper face $\tau=\left[v_{0}, \ldots, v_{k}\right]$ of $\sigma, 0 \leq k<n$, and of different permutations $\pi, \pi^{\prime}$ of the set $\{k+1, \ldots, n\}$. This case is settled by the following lemma.

Lemma 3.9. Let $\tau=\left[v_{0}, \ldots, v_{k}\right], 0 \leq k<n$, and let $\pi, \pi^{\prime}$ be different permutations of the set $\{k+1, \ldots, n\}$. Then the intersection $c_{\pi}^{\tau} \cap c_{\pi^{\prime}}^{\tau}$ of the $n$-cells $c_{\pi}^{\tau}=c^{\tau} \oplus d_{\pi}^{\tau}$ and $c_{\pi^{\prime}}^{\tau}=c^{\tau} \oplus d_{\pi^{\prime}}^{\tau}$ is a common face of both $n$-cells $c_{\pi}^{\tau}$ and $c_{\pi^{\prime}}^{\tau}$. More precisely,

$$
c_{\pi}^{\tau} \cap c_{\pi^{\prime}}^{\tau}=c^{\tau} \oplus\left(d_{\pi}^{\tau} \cap d_{\pi^{\prime}}^{\tau}\right) .
$$

Proof. Note that $w_{0}^{\tau}=w_{0}^{0 \ldots k}$ is a vertex of $c^{\tau} \subseteq \operatorname{Aff}\left(c^{\tau}\right)$ and by (3.19) and (3.20), $w_{0}^{\tau}$ is also a vertex of $d_{\pi}^{\tau} \cap d_{\pi^{\prime}}^{\tau} \subseteq \operatorname{Aff}\left(d_{\pi}^{\tau} \cap d_{\pi^{\prime}}^{\tau}\right)$. Since $\operatorname{Aff}\left(c^{\tau}\right) \cap$ $\operatorname{Aff}\left(d_{\pi}^{\tau} \cap d_{\pi^{\prime}}^{\tau}\right) \subseteq \operatorname{Aff}\left(c^{\tau}\right) \cap \operatorname{Aff}\left(d_{\pi}^{\tau}\right)=\left\{w_{0}^{\tau}\right\}$, it follows that $c^{\tau} \oplus\left(d_{\pi}^{\tau} \cap d_{\pi^{\prime}}^{\tau}\right)$ is well defined and has $w_{0}^{0 \ldots k}$ for its reference point. By Lemma 3.7, $d_{\pi}^{\tau} \cap d_{\pi^{\prime}}^{\tau}$ is a face of $d_{\pi}^{\tau}$ and thus, $c^{\tau} \oplus\left(d_{\pi}^{\tau} \cap d_{\pi^{\prime}}^{\tau}\right)=c^{\tau}+\left(d_{\pi}^{\tau} \cap d_{\pi^{\prime}}^{\tau}\right)-w_{0}^{0 \ldots k}$ is a face of $c^{\tau}+d_{\pi}^{\tau}-w_{0}^{0 \ldots k}=c_{\pi}^{\tau}$. Analogously, $c^{\tau} \oplus\left(d_{\pi}^{\tau} \cap d_{\pi^{\prime}}^{\tau}\right)$ is a face of $c_{\pi^{\prime}}^{\tau}$.

To complete the proof, we need to prove (3.47). Consider an arbitrary point $u \in c_{\pi}^{\tau} \cap c_{\pi^{\prime}}^{\tau}$. It is of the form

$$
u=s+t-w_{0}^{0 \ldots k}=s^{\prime}+t^{\prime}-w_{0}^{0 \ldots k},
$$

where $s, s^{\prime} \in c^{\tau}, t \in d_{\pi}^{\tau}$ and $t^{\prime} \in d_{\pi^{\prime}}^{\tau}$. Consider the affine mapping $p_{\tau \sigma}: \sigma \rightarrow \tau$, determined by its values at the vertices of $\sigma$,

$$
p_{\tau \sigma}\left(v_{j}\right)= \begin{cases}v_{j}, & 0 \leq j \leq k, \\ b^{\tau}, & k+1 \leq j \leq n .\end{cases}
$$

Note that $p_{\tau \sigma}$ uniquely extends to an affine mapping $p_{\tau \sigma}$ : $\operatorname{Aff}(\sigma) \rightarrow \operatorname{Aff}(\tau)$. Since $p_{\tau \sigma}$ is a retraction, an application of the affine mapping $p_{\tau \sigma}$ to $(3.48)$ yields the equality

$$
s+p_{\tau \sigma}(t)=s^{\prime}+p_{\tau \sigma}\left(t^{\prime}\right)
$$

Let us show that

$$
p_{\tau \sigma}(t)=w_{0}^{0 \ldots k}=p_{\tau \sigma}\left(t^{\prime}\right)
$$

and thus, $s=s^{\prime}$. Indeed, $t \in d_{\pi}^{\tau}=\left[w_{0}^{0 \ldots k}, \ldots, w_{0}^{0 \ldots k \pi(k+1) \ldots \pi(k+i)}, \ldots, w_{0}^{0 \ldots n}\right]$ and $w_{0}^{0 \ldots k \pi(k+1) \ldots \pi(k+i)}=\frac{1}{2}\left(v_{0}+b^{0 \ldots k \pi(k+1) \ldots \pi(k+i)}\right)$. Moreover,

$$
b^{0 \ldots k \pi(k+1) \ldots \pi(k+i)}=\frac{1}{k+1+i}\left(v_{0}+\ldots+v_{k}+v_{\pi(k+1)}, \ldots,+v_{\pi(k+i)}\right)
$$

and thus,

$$
\begin{aligned}
p_{\tau \sigma}\left(b^{0 \ldots k \pi(k+1) \ldots \pi(k+i)}\right) & =\frac{1}{k+1+i}\left(v_{0}+\ldots+v_{k}\right)+\frac{1}{k+1+i}\left(b^{\tau}+\ldots+b^{\tau}\right) \\
& =\frac{k+1}{k+1+i} b^{\tau}+\frac{i}{k+1+i} b^{\tau}=b^{\tau}
\end{aligned}
$$


Consequently,

$$
\begin{aligned}
p_{\tau \sigma}\left(w_{0}^{0 \ldots k \pi(k+1) \ldots \pi(k+i)}\right) & =\frac{1}{2}\left(v_{0}+p_{\tau \sigma}\left(b^{0 \ldots k \pi(k+1) \ldots \pi(k+i)}\right)\right) \\
& =\frac{1}{2}\left(v_{0}+b^{\tau}\right)=w_{0}^{0 \ldots k}
\end{aligned}
$$

and we see that $p_{\tau \sigma}$ maps all vertices of $d_{\pi}^{\tau}$ to the point $w_{0}^{0 \ldots k}$. Therefore, it also maps $t$ to that point, i.e., $p_{\tau \sigma}(t)=w_{0}^{0 \ldots k}$. In the same way one verifies that $p_{\tau \sigma}\left(t^{\prime}\right)=w_{0}^{0 \ldots k}$. Now $s=s^{\prime}$ and (3.48) yield the conclusion that also $t=t^{\prime} \in d_{\pi}^{\tau} \cap d_{\pi^{\prime}}^{\tau}$. All this proves that $u=s+t-w_{0}^{0 \ldots k} \in c^{\tau}+\left(d_{\pi}^{\tau} \cap d_{\pi^{\prime}}^{\tau}\right)-w_{0}^{0 \ldots k}$ and thus,

$$
c_{\pi}^{\tau} \cap c_{\pi^{\prime}}^{\tau} \subseteq c^{\tau}+\left(d_{\pi}^{\tau} \cap d_{\pi^{\prime}}^{\tau}\right)-w_{0}^{0 \ldots k} .
$$

The opposite inclusion is obvious and we see that $c_{\pi}^{\tau} \cap c_{\pi^{\prime}}^{\tau}=c^{\tau}+\left(d_{\pi}^{\tau} \cap d_{\pi^{\prime}}^{\tau}\right)-$ $w_{0}^{0 \ldots k}$, which establishes (3.47).

3.7.2. Case $(b), \tau^{\prime} \neq \tau^{\prime \prime}$ and $\bar{\tau}=\sigma$. If $\tau^{\prime} \cap \tau^{\prime \prime} \neq \emptyset$, the intersection $\tau^{\prime} \cap \tau^{\prime \prime}$ is a simplex $\tau$, which is the common face of $\tau^{\prime}$ and $\tau^{\prime \prime}$. There is no loss of generality in assuming that $\tau=\left[v_{0}, \ldots, v_{k}\right]$, where $0 \leq k<n$. At least one of the sets $\tau^{\prime} \backslash \tau, \tau^{\prime \prime} \backslash \tau$ must be nonempty, for otherwise, one would have $\tau^{\prime}=\tau=\tau^{\prime \prime}$. It suffices to consider the case when $\tau^{\prime} \backslash \tau \neq \emptyset$. If $\tau^{\prime} \neq \sigma$, i.e., if it is a proper face of $\sigma$, there is no loss of generality in assuming that $\tau=\left[v_{0}, \ldots, v_{k}\right], \tau^{\prime}=\left[v_{0}, \ldots, v_{k^{\prime}}\right], \tau^{\prime \prime}=\left[v_{0}, \ldots, v_{k}, v_{k^{\prime}+1}, \ldots, v_{n}\right]$, where $0 \leq k<k^{\prime}<n$. If $\tau^{\prime}=\sigma$, then $c_{\pi^{\prime}}^{\tau^{\prime}}=c^{\sigma}$ is the central $n$-simplex, $\tau^{\prime \prime}=\tau$ and $\pi^{\prime \prime}$ is a permutation of the set $\{k+1, \ldots, n\}$. Renaming $\pi^{\prime \prime}$ to $\pi$ the $n$-cell $c_{\pi^{\prime \prime}}^{\tau^{\prime \prime}}$ becomes $c_{\pi}^{\tau}$. Therefore, case $(b)$ is settled by the following lemma.

LEMMA 3.10 .

(i) Let $\tau^{\prime}=\left[v_{0}, \ldots, v_{k^{\prime}}\right], \tau^{\prime \prime}=\left[v_{0}, \ldots, v_{k}, v_{k^{\prime}+1}, \ldots, v_{n}\right], \tau^{\prime} \cap \tau^{\prime \prime}=\tau=$ $\left[v_{0}, \ldots, v_{k}\right], 0 \leq k<k^{\prime}<n$ and let $c_{\pi^{\prime}}^{\tau^{\prime}}, c_{\pi^{\prime \prime}}^{\tau^{\prime \prime}}$ be $n$-cells from $L(\sigma)$. Then the intersection $c_{\pi^{\prime}}^{\tau^{\prime}} \cap c_{\pi^{\prime \prime}}^{\tau^{\prime \prime}}$ is a common face of $c_{\pi^{\prime}}^{\tau^{\prime}}$ and $c_{\pi^{\prime \prime}}^{\tau^{\prime \prime}}$. Moreover,

$$
c_{\pi^{\prime}}^{\tau^{\prime}} \cap c_{\pi^{\prime \prime}}^{\tau^{\prime \prime}}=\left[w_{0}^{\sigma}, \ldots, w_{k}^{\sigma}\right] .
$$

(ii) Let $\tau=\left[v_{0}, \ldots, v_{k}\right], 0 \leq k<n$ and let $c_{\pi}^{\tau}$ be a peripheral $n$-cell from $L(\sigma)$. Then the intersection $c^{\sigma} \cap c_{\pi}^{\tau}$ of the central n-simplex $c^{\sigma}$ with the $n$-cell $c_{\pi}^{\tau}$ is a common face of $c^{\sigma}$ and $c_{\pi}^{\tau}$. Moreover,

$$
c^{\sigma} \cap c_{\pi}^{\tau}=\left[w_{0}^{\sigma}, \ldots, w_{k}^{\sigma}\right] .
$$

(iii) Let $c_{\pi^{\prime}}^{\tau^{\prime}}$ and $c_{\pi^{\prime \prime}}^{\tau^{\prime \prime}}$ be $n$-cells from $L(\sigma)$ such that $\bar{\tau}=\sigma$. If $\tau^{\prime} \cap \tau^{\prime \prime}=\emptyset$, then also $c_{\pi^{\prime}}^{\tau^{\prime}} \cap c_{\pi^{\prime \prime}}^{\tau^{\prime \prime}}=\emptyset$.

Proof. Case $(i)$. In the $n$-dimensional affine manifold $S_{n}=\operatorname{Aff}(\sigma) \subseteq V$ we will define an $(n-1)$-dimensional affine manifold $S$ such that $S$ is a supporting hyperplane for both convex sets $c_{\pi^{\prime}}^{\tau^{\prime}}$ and $c_{\pi^{\prime \prime}}^{\tau^{\prime \prime}}$. Moreover, $S$ will separate these two sets, i.e., they will lie in different subspaces $S^{+}, S^{-}$, determined by 
$S$. Since $S^{+} \cap S^{-}=S$, it will follow that $c_{\pi^{\prime}}^{\tau^{\prime}} \cap c_{\pi^{\prime \prime}}^{\tau^{\prime \prime}}=c_{\pi^{\prime}}^{\tau^{\prime}} \cap c_{\pi^{\prime \prime}}^{\tau^{\prime \prime}} \cap S$. We will also show that

$$
\begin{gathered}
c_{\pi^{\prime}}^{\tau^{\prime}} \cap S=\left[w_{0}^{\sigma}, \ldots, w_{k}^{\sigma}\right], \\
c_{\pi^{\prime \prime}}^{\tau^{\prime \prime}} \cap S=\left[w_{0}^{\sigma}, \ldots, w_{k}^{\sigma}, w_{k^{\prime}+1}^{\sigma}, \ldots, w_{n}^{\sigma}\right],
\end{gathered}
$$

which will imply $c_{\pi^{\prime}}^{\tau^{\prime}} \cap c_{\pi^{\prime \prime}}^{\tau^{\prime \prime}}=\left[w_{0}^{\sigma}, \ldots, w_{k}^{\sigma}\right]$, as asserted by (3.56).

To define $S, S^{+}$and $S^{-}$, we consider the function $\Lambda: \operatorname{Aff}(\sigma) \rightarrow \mathbb{R}$, given by the formula

$$
\Lambda(u)=\lambda_{k+1}(u)+\ldots+\lambda_{k^{\prime}}(u), u \in \operatorname{Aff}(\sigma)
$$

and we put

$$
\begin{aligned}
& S=\left\{u \in \operatorname{Aff}(\sigma) \mid \Lambda(u)=\frac{k^{\prime}-k}{2(n+1)}\right\}, \\
& S^{+}=\left\{u \in \operatorname{Aff}(\sigma) \mid \Lambda(u) \geq \frac{k^{\prime}-k}{2(n+1)}\right\}, \\
& S^{-}=\left\{u \in \operatorname{Aff}(\sigma) \mid \Lambda(u) \leq \frac{k^{\prime}-k}{2(n+1)}\right\} .
\end{aligned}
$$

We will show that, for all vertices $w^{\prime}$ of $c_{\pi^{\prime}}^{\tau^{\prime}}$, one has $\Lambda\left(w^{\prime}\right) \geq \frac{k^{\prime}-k}{2(n+1)}$, which implies $\Lambda(u) \geq \frac{k^{\prime}-k}{2(n+1)}$, for all points $u \in c_{\pi^{\prime}}^{\tau^{\prime}}$, i.e., $c_{\pi^{\prime}}^{\tau^{\prime}} \subseteq S^{+}$. Moreover, of all the vertices $w^{\prime}$ only $w_{0}^{0 \ldots n}, \ldots, w_{k}^{0 \ldots n}$ satisfy the equality $\Lambda\left(w^{\prime}\right)=\frac{k^{\prime}-k}{2(n+1)}$. Therefore, $S$ is a supporting hyperplane for the convex set $c_{\pi^{\prime}}^{\tau^{\prime}}$ and (3.58) holds. Similarly, we will show that, for all vertices $w^{\prime \prime}$ of $c_{\pi^{\prime \prime}}^{\tau^{\prime \prime}}$, one has $\Lambda\left(w^{\prime \prime}\right) \leq \frac{k^{\prime}-k}{2(n+1)}$ and only the vertices $w^{\prime \prime}$ from the set $\left\{w_{0}^{\sigma}, \ldots, w_{k}^{\sigma}, w_{k^{\prime}+1}^{\sigma}, \ldots, w_{n}^{\sigma}\right\}$ satisfy the equality $\Lambda\left(w^{\prime}\right)=\frac{k^{\prime}-k}{2(n+1)}$. Therefore, $c_{\pi^{\prime \prime}}^{\tau^{\prime \prime}} \subseteq S^{-}$and (3.59) holds.

According to (3.11), the vertices of $c_{\pi^{\prime}}^{\tau^{\prime}}$ are the points $w_{m}^{0 \ldots k^{\prime}}$ and the points $w_{m}^{0 \ldots k^{\prime} \pi^{\prime}\left(k^{\prime}+1\right) \ldots \pi^{\prime}\left(k^{\prime}+i\right)}$, where $1 \leq i \leq n-k^{\prime}$ and $0 \leq m \leq k^{\prime}$. Note that (3.62)

$$
\begin{aligned}
& b^{0 \ldots k^{\prime} \pi^{\prime}}\left(k^{\prime}+1\right) \ldots \pi^{\prime}\left(k^{\prime}+i\right)= \\
& \frac{1}{k^{\prime}+1+i}\left(v_{0}+\ldots+v_{k}+v_{k+1}+\ldots+v_{k^{\prime}}+v_{\pi^{\prime}\left(k^{\prime}+1\right)}+\ldots+v_{\pi^{\prime}\left(k^{\prime}+i\right)}\right) .
\end{aligned}
$$

Since $\{0, \ldots, k\} \cap\left\{k+1, \ldots, k^{\prime}\right\}=\emptyset$ and $\left\{\pi^{\prime}\left(k^{\prime}+1\right), \ldots, \pi^{\prime}\left(k^{\prime}+i\right)\right\} \cap\{k+$ $\left.1, \ldots, k^{\prime}\right\}=\emptyset$, we see that $\Lambda\left(v_{0}+\ldots+v_{k}\right)=\Lambda\left(v_{\pi^{\prime}\left(k^{\prime}+1\right)}+\ldots+v_{\pi^{\prime}\left(k^{\prime}+i\right)}\right)=0$ and thus,

$$
\begin{aligned}
& \Lambda\left(b^{0 \ldots k^{\prime} \pi^{\prime}\left(k^{\prime}+1\right) \ldots \pi^{\prime}\left(k^{\prime}+i\right)}\right)=\frac{1}{k^{\prime}+1+i} \Lambda\left(v_{k+1}+\ldots+v_{k^{\prime}}\right)= \\
& \frac{1}{k^{\prime}+1+i}\left(\lambda_{k+1}\left(v_{k+1}\right)+\ldots+\lambda_{k^{\prime}}\left(v_{k^{\prime}}\right)\right)=\frac{k^{\prime}-k}{k^{\prime}+1+i} .
\end{aligned}
$$

Now note that $\Lambda\left(v_{m}\right)=0$, when $0 \leq m \leq k$ and $\Lambda\left(v_{m}\right)=1$, when $k+1 \leq m \leq$ $k^{\prime}$. Since $w_{m}^{0 \ldots k^{\prime} \pi^{\prime}\left(k^{\prime}+1\right) \ldots \pi^{\prime}\left(k^{\prime}+i\right)}=\frac{1}{2} v_{m}+\frac{1}{2} b^{0 \ldots k^{\prime} \pi^{\prime}\left(k^{\prime}+1\right) \ldots \pi^{\prime}\left(k^{\prime}+i\right)}$, we conclude that

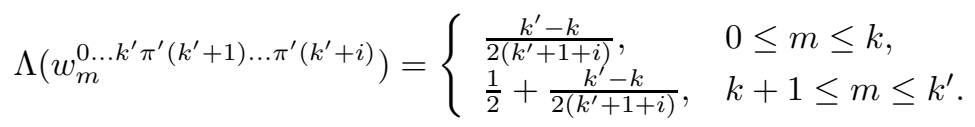


A simplified version of the above calculation gives for $\Lambda\left(w_{m}^{0 \ldots k^{\prime}}\right)$ the value obtained by substituting $i=0$ in (3.64). For $i=n-k^{\prime}$, (3.64) yields

$$
\begin{gathered}
\Lambda\left(w_{0}^{\sigma}\right)=\ldots=\Lambda\left(w_{k}^{\sigma}\right)=\frac{k^{\prime}-k}{2(n+1)}, \\
\Lambda\left(w_{k+1}^{\sigma}\right)=\ldots=\Lambda\left(w_{k^{\prime}}^{\sigma}\right)=\frac{1}{2}+\frac{k^{\prime}-k}{2(n+1)},
\end{gathered}
$$

because $\left\{\pi^{\prime}\left(k^{\prime}+1\right), \ldots, \pi^{\prime}(n)\right\}=\left\{k^{\prime}+1, \ldots, n\right\}$. Moreover, $i<n-k^{\prime}$ implies $\frac{k^{\prime}-k}{k^{\prime}+1+i}>\frac{k^{\prime}-k}{n+1}$, which shows that $\Lambda$ assumes the minimal value $\frac{k^{\prime}-k}{2(n+1)}$ only for the vertices $w_{0}^{\sigma}, \ldots, w_{k}^{\sigma}$. We have thus, verified the above made assertions concerning the vertices of $c_{\pi^{\prime}}^{\tau^{\prime}}$.

A similar calculation establishes the assertions for the vertices of $c_{\pi^{\prime \prime}}^{\tau^{\prime \prime}}$. These are the points $w_{m}^{0 \ldots k k^{\prime}+1 \ldots n}$ and $w_{m}^{0 \ldots k k^{\prime}+1 \ldots n \pi^{\prime \prime}(k+1) \ldots \pi^{\prime \prime}(k+j)}$, where $1 \leq$ $j \leq k^{\prime}-k$ and $0 \leq m \leq k$ or $k^{\prime}+1 \leq m \leq n$. Note that

$$
\begin{aligned}
& b^{0 \ldots k k^{\prime}+1 \ldots n \pi^{\prime \prime}(k+1) \ldots \pi^{\prime \prime}(k+j)}= \\
& \frac{1}{k+1+n-k^{\prime}+j}\left(v_{0}+\ldots+v_{k}+v_{k^{\prime}+1}+\ldots+v_{n}+v_{\pi^{\prime \prime}(k+1)}+\ldots+v_{\pi^{\prime \prime}(k+j)}\right) .
\end{aligned}
$$

It is readily seen that $\Lambda\left(v_{0}+\ldots+v_{k}\right)=0$ and $\Lambda\left(v_{k^{\prime}+1}+\ldots+v_{n}\right)=0$. Since $\pi^{\prime \prime}(k+1), \ldots, \pi^{\prime \prime}(k+j)$ are $j$ different elements of the set $\left\{k+1, \ldots, k^{\prime}\right\}$, one has $\Lambda\left(v_{\pi^{\prime \prime}(k+1)}\right)=\ldots=\Lambda\left(v_{\pi^{\prime \prime}(k+j)}\right)=1$ and thus, $\Lambda\left(v_{\pi^{\prime \prime}(k+1)}+\ldots+\right.$ $\left.v_{\pi^{\prime \prime}(k+j)}\right)=j$. Consequently,

$$
\Lambda\left(b^{0 \ldots k k^{\prime}+1 \ldots n \pi^{\prime \prime}(k+1) \ldots \pi^{\prime \prime}(k+j)}\right)=\frac{j}{k+1+n-k^{\prime}+j} .
$$

Since $\Lambda\left(v_{m}\right)=0$, for $0 \leq m \leq k$ or $k^{\prime}+1 \leq m \leq n$, we conclude that

$$
\Lambda\left(w_{m}^{0 \ldots k k^{\prime}+1 \ldots n \pi^{\prime \prime}(k+1) \ldots \pi^{\prime \prime}(k+j)}\right)=\frac{j}{2\left(k+1+n-k^{\prime}+j\right)} .
$$

Moreover, it is readily seen that

$$
\Lambda\left(w_{m}^{0 \ldots k k^{\prime}+1 \ldots n}\right)=0 .
$$

For $j=k^{\prime}-k,(3.69)$ becomes

$$
\Lambda\left(w_{m}^{\sigma}\right)=\frac{k^{\prime}-k}{2(n+1)},
$$

because $\pi^{\prime \prime}\left\{k+1, \ldots, k^{\prime}\right\}=\left\{k+1, \ldots, k^{\prime}\right\}$ and thus, $w_{m}^{0 \ldots k k^{\prime}+1 \ldots n k+1 \ldots k^{\prime}}=w_{m}^{\sigma}$. Furthermore, for $1 \leq j<k^{\prime}-k$, one has $\frac{j}{2\left(k+1+n-k^{\prime}+j\right)}<\frac{k^{\prime}-k}{2(n+1)}$, because $k^{\prime}<n$ implies $0<k+1+n-k^{\prime}$ and $\frac{j}{\left(k+1+n-k^{\prime}+j\right)}<\frac{j+1}{\left(k+1+n-k^{\prime}+j+1\right)}$. This shows that the right side of (3.69) assumes its maximal value $\frac{k^{\prime}-k}{2(n+1)}$ only for the vertices $w_{0}^{\sigma}, \ldots, w_{k}^{\sigma}, w_{k^{\prime}+1}^{\sigma}, \ldots, w_{n}^{\sigma}$. Hence, we have verified the above made assertions concerning the vertices of $c_{\pi^{\prime \prime}}^{\tau^{\prime \prime}}$.

Clearly, $\left[w_{0}^{\sigma}, \ldots, w_{k}^{\sigma}\right]$ is a face of $\left[w_{0}^{\sigma}, \ldots, w_{k^{\prime}}^{\sigma}\right]$. However, the latter simplex is spanned by the last row of (3.11) for $c_{\pi^{\prime}}^{\tau^{\prime}}$ and thus, it is a face of $c_{\pi^{\prime}}^{\tau^{\prime}}$. 
Consequently, $\left[w_{0}^{\sigma}, \ldots, w_{k}^{\sigma}\right] \leq c_{\pi^{\prime}}^{\tau^{\prime}}$. Analogously, $\left[w_{0}^{\sigma}, \ldots, w_{k}^{\sigma}\right]$ is a face of the last row of (3.11) for $c_{\pi^{\prime \prime}}^{\tau^{\prime \prime}}$ and thus, it is also a face of $c_{\pi^{\prime \prime}}^{\tau^{\prime \prime}}$.

Case (ii). The proof of (3.57) can be obtained from the above proof of (3.56) by replacing everywhere $k^{\prime}$ by $n$ and $i$ by 0 and by deleting expressions which in this case make no sense, like $k^{\prime}+1 \ldots n, \pi^{\prime}\left(k^{\prime}+1\right) \ldots \pi^{\prime}\left(k^{\prime}+i\right)$ or $v_{k^{\prime}+1}+\ldots+v_{n}$. Then $\tau^{\prime}=\left[v_{0}, \ldots, v_{k^{\prime}}\right]$ becomes $\sigma=\left[v_{0}, \ldots, v_{k^{\prime}}\right], \tau^{\prime \prime}=$ $\left[v_{0}, \ldots, v_{k}, v_{k^{\prime}+1}, \ldots, v_{n}\right]$ becomes $\tau=\left[v_{0}, \ldots, v_{k}\right]$ and $c_{\pi^{\prime}}^{\tau^{\prime}}$ and $c_{\pi^{\prime \prime}}^{\tau^{\prime \prime}}$ become $c^{\sigma}$ and $c_{\pi}^{\tau}$, respectively. Furthermore, $\Lambda(u)$ becomes the sum $\lambda_{k+1}(u)+\ldots+\lambda_{n}(u)$ and the expression $\frac{k^{\prime}-k}{2(n+1)}$ in (3.61) becomes $\frac{n-k}{2(n+1)}$. The vertices of $c^{\sigma}$ are the points $w_{m}^{\sigma}$, where $0 \leq m \leq n$ and

$$
\Lambda\left(w_{m}^{\sigma}\right)= \begin{cases}\frac{n-k}{2(n+1)}, & 0 \leq m \leq k, \\ \frac{1}{2}+\frac{n-k}{2(n+1)}, & k+1 \leq m \leq n .\end{cases}
$$

Hence, $\Lambda$ assumes its minimal value $\frac{n-k}{2(n+1)}$ at the vertices $w_{0}^{\sigma}, \ldots, w_{k}^{\sigma}$ and thus, $c^{\sigma} \subseteq S^{+}$and $c^{\sigma} \cap S=\left[w_{0}^{\sigma}, \ldots, w_{k}^{\sigma}\right]$. The vertices of $c_{\pi}^{\tau}$ are the points $w_{m}^{0 \ldots k}$ and $w_{m}^{0 \ldots k \pi(k+1) \ldots \pi(k+j)}$, where $1 \leq j \leq n-k$ and $0 \leq m \leq k$. Furthermore, $\Lambda\left(w_{m}^{0 \ldots k}\right)=0$ and

$$
\Lambda\left(w_{m}^{0 \ldots k \pi(k+1) \ldots \pi(k+j)}\right)=\frac{j}{2(k+1+j)} .
$$

The right side of (3.73) assumes its maximal value $\frac{n-k}{2(n+1)}$ only for the vertices $w_{0}^{\sigma}, \ldots, w_{k}^{\sigma}$. Therefore, $c_{\pi}^{\tau} \subseteq S^{-}, c_{\pi}^{\tau} \cap S=\left[w_{0}^{\sigma}, \ldots, w_{k}^{\sigma}\right]$ and the assertion (3.57) follows. That $\left[w_{0}^{\sigma}, \ldots, w_{k}^{\sigma}\right]$ is a face of $c_{\pi}^{\tau}$ follows as in the above case. That it is a face of $c^{\sigma}$ is an immediate consequence of the definition of $c^{\sigma}$.

Case (iii). If $\tau^{\prime} \cap \tau^{\prime \prime}=\emptyset$, there is no loss of generality in assuming that $\tau^{\prime}=\left[v_{0}, \ldots, v_{k}\right]$ and $\tau^{\prime \prime}=\left[v_{k+1}, \ldots, v_{n}\right]$, where $0 \leq k<n$. In this case we define $\Lambda$ by putting $\Lambda(u)=\lambda_{0}(u)+\ldots \lambda_{k}(u)$. In (3.61) we replace the expression $\frac{k^{\prime}-k}{2(n+1)}$ by $\frac{k+1}{2(n+1)}$. The vertices of $c_{\pi^{\prime}}^{\tau^{\prime}}$ are now the points $w_{m}^{0 \ldots k}$ and $w_{m}^{0 \ldots k \pi^{\prime}(k+1) \ldots \pi^{\prime}(k+i)}$, where $1 \leq i \leq n-k$ and $0 \leq m \leq k$. A computation like the previous ones shows that

$$
\Lambda\left(w_{m}^{0 \ldots k \pi^{\prime}(k+1) \ldots \pi^{\prime}(k+i)}\right)=\frac{1}{2}+\frac{k+1}{2(k+1+i)}
$$

and $\Lambda\left(w_{m}^{0 \ldots k}\right)=1$. Consequently, the minimal value of $\Lambda$ at the vertices of $c_{\pi^{\prime}}^{\tau^{\prime}}$ is $\frac{1}{2}+\frac{k+1}{2(n+1)}$ and thus, for $u \in c_{\pi^{\prime}}^{\tau^{\prime}}$, one has

$$
\frac{1}{2}+\frac{k+1}{2(n+1)} \leq \Lambda(u)
$$

Furthermore, the vertices of $c_{\pi^{\prime \prime}}^{\tau^{\prime \prime}}$ are the points $w_{m}^{k+1 \ldots n}$ and $w_{m}^{k+1 \ldots n \pi^{\prime \prime}(0) \ldots \pi^{\prime \prime}(j)}$, where $0 \leq j \leq k$ and $k+1 \leq m \leq n$. A computation like the previous ones shows that

$$
\Lambda\left(w_{m}^{k+1 \ldots n \pi^{\prime \prime}(0) \ldots \pi^{\prime \prime}(j)}\right)=\frac{j+1}{2(n-k+j+1)}
$$


and $\Lambda\left(w_{m}^{k+1 \ldots n}\right)=\frac{1}{2(n-k+1)}$. Consequently, the maximal value of $\Lambda$ at the vertices of $c_{\pi^{\prime \prime}}^{\tau^{\prime \prime}}$ is $\frac{k+1}{2(n+1)}$ and thus, for $u \in c_{\pi^{\prime \prime}}^{\tau^{\prime \prime}}$, one has

$$
\Lambda(u) \leq \frac{k+1}{2(n+1)} .
$$

Since there is no point $u$, which satisfies both inequalities (3.75) and (3.77), it follows that $c_{\pi^{\prime}}^{\tau^{\prime}} \cap c_{\pi^{\prime \prime}}^{\tau^{\prime \prime}}=\emptyset$.

3.7.3. Case $(c) . \tau^{\prime} \neq \tau^{\prime \prime}$ and $\bar{\tau}=\operatorname{Conv}\left(\tau^{\prime} \cup \tau^{\prime \prime}\right)<\sigma$.

3.7.3.1. We first prove a lemma, which will enable us to reduce the proof in case $(c)$ to case $(b)$, using the affine mapping $p_{\tau \sigma}: \sigma \rightarrow \tau$, determined by (3.49).

Lemma 3.11. Let $\tau=\left[v_{0}, \ldots, v_{k}\right], \bar{\tau}=\left[v_{0}, \ldots, v_{\bar{k}}\right]$ be faces of $\sigma$, where $0 \leq k<\bar{k}<n$.

(i) For every $n$-cell $c_{\pi}^{\tau} \in L(\sigma)$, there is a permutation $\rho$ of the set $\{k+$ $1, \ldots, \bar{k}\}$ such that the corresponding $\bar{k}$-cell $c_{\rho}^{\tau} \in L(\bar{\tau})$ has the property that

$$
p_{\bar{\tau} \sigma}\left(c_{\pi}^{\tau}\right) \subseteq c_{\rho}^{\tau} .
$$

There is also a permutation $\vartheta$ of the set $\{\bar{k}+1, \ldots, n\}$ and there is a common face $c_{\pi \vartheta}^{\tau \bar{\tau}}$ of $c_{\pi}^{\tau}$ and $c_{\vartheta}^{\bar{\tau}}$ such that

$$
\left(p_{\bar{\tau} \sigma} \mid c_{\pi}^{\tau}\right)^{-1}\left[w_{0}^{\bar{\tau}}, \ldots, w_{k}^{\bar{\tau}}\right]=c_{\pi \vartheta}^{\tau \bar{\tau}} .
$$

(ii) For every $n$-cell $c_{\frac{\pi}{\pi}}^{\bar{\tau}} \in L(\sigma)$,

$$
p_{\bar{\tau} \sigma}\left(c_{\bar{\pi}}^{\bar{\tau}}\right) \subseteq c^{\bar{\tau}}
$$

and there is a face $c_{\bar{\pi}}^{\tau \bar{\tau}}$ of $c_{\frac{\pi}{\pi}}^{\bar{\tau}}$ such that

$$
\left(p_{\bar{\tau} \sigma} \mid c_{\bar{\pi}}^{\bar{\tau}}\right)^{-1}\left[w_{0}^{\bar{\tau}}, \ldots, w_{k}^{\bar{\tau}}\right]=c_{\bar{\pi}}^{\tau \bar{\tau}} .
$$

Proof. (i) Since $\pi:\{k+1, \ldots, n\} \rightarrow\{k+1, \ldots, n\}$ is a bijection and $\{k+1, \ldots, \bar{k}\}$ is a proper subset of $\{k+1, \ldots, n\}$, there are precisely $\bar{k}-k$ integers $j$ such that $1 \leq j \leq n-k$ and $\pi(k+j) \in\{k+1, \ldots, \bar{k}\}$ and there are precisely $n-\bar{k}$ integers $l$ such that $1 \leq l \leq n-k$ and $\pi(k+l) \in\{\bar{k}+1, \ldots, n\}$. Denote these integers by $1 \leq j_{1}<\ldots<j_{a}<\ldots<j_{\bar{k}-k} \leq n-k$ and $1 \leq l_{1}<$ $\ldots<l_{b}<\ldots<l_{n-\bar{k}} \leq n-k$, respectively. Note that $k+1 \leq \pi\left(k+j_{a}\right) \leq \bar{k}$ and $n \geq \pi\left(k+l_{b}\right) \geq \bar{k}+1$. Define functions $\rho:\{k+1, \ldots, \bar{k}\} \rightarrow\{k+1, \ldots, \bar{k}\}$ and $\vartheta:\{\bar{k}+1, \ldots, n\} \rightarrow\{\bar{k}+1, \ldots, n\}$ by putting

$$
\begin{aligned}
& \rho(k+a)=\pi\left(k+j_{a}\right), 1 \leq a \leq \bar{k}-k, \\
& \vartheta(\bar{k}+b)=\pi\left(k+l_{b}\right), 1 \leq b \leq n-\bar{k} .
\end{aligned}
$$

The functions $\rho$ and $\vartheta$ are permutations, because they are surjective and injective. 
Let us now prove (3.78). Every point $u \in c_{\pi}^{\tau}$ is of the form $u=s+t-w_{0}^{\tau}$, where $s \in c^{\tau}$ and $t \in d_{\pi}^{\tau}$. Since $\left\{s, w_{0}^{\tau}\right\} \subseteq c^{\tau} \subseteq \tau \subseteq \bar{\tau}$ and $p_{\bar{\tau} \sigma}: \sigma \rightarrow \bar{\tau}$ is a retraction, it follows that $p_{\bar{\tau} \sigma}(s)=s$ and $p_{\bar{\tau} \sigma}\left(w_{0}^{\tau}\right)=w_{0}^{\tau}$. Therefore, to prove (3.78), it suffices to show that $p_{\bar{\tau} \sigma}(t) \in d_{\rho}^{\tau}$, because then, $p_{\bar{\tau} \sigma}(u)=$ $s+p_{\bar{\tau} \sigma}(t)-w_{0}^{\tau} \in c^{\tau}+d_{\rho}^{\tau}-w_{0}^{\tau}=c_{\rho}^{\tau}$.

To prove that $p_{\bar{\tau} \sigma}(t) \in d_{\rho}^{\tau}$, it suffices to show that $p_{\bar{\tau} \sigma}$ maps the vertices of $d_{\pi}^{\tau}$ into

$$
\begin{aligned}
d_{\rho}^{\tau} & =\left[w_{0}^{0 \ldots k}, \ldots, w_{0}^{0 \ldots k \rho(k+1) \ldots \rho(k+i)}, \ldots, w_{0}^{0 \ldots . \bar{k}}\right] \\
& =\left[w_{0}^{0 \ldots k}, \ldots, w_{0}^{0 \ldots k \pi\left(k+j_{1}\right) \ldots \pi\left(k+j_{i}\right)}, \ldots, w_{0}^{0 \ldots \bar{k}}\right] .
\end{aligned}
$$

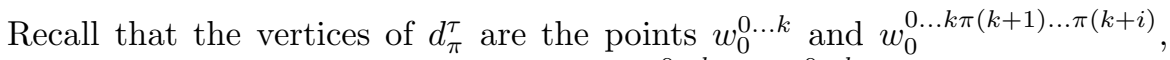
$1 \leq i \leq n-k$. We already saw that $p_{\bar{\tau} \sigma}\left(w_{0}^{0 \ldots k}\right)=w_{0}^{0 \ldots k} \in d_{\rho}^{\tau}$. To determine $p_{\bar{\tau} \sigma}\left(w_{0}^{0 \ldots k \pi(k+1) \ldots \pi(k+i)}\right)$, note that the set $\{1, \ldots, n-k\}$ is the disjoint sum of the sets $\left\{j_{1}, \ldots, j_{\bar{k}-k}\right\}$ and $\left\{l_{1}, \ldots, l_{n-\bar{k}}\right\}$. Let $a(i)$ and $b(i)$ be the largest integers $a$ and $b$ such that $j_{a} \leq i$ and $l_{b} \leq i$, respectively. Clearly,

$$
\{1, \ldots, i\}=\left\{j_{1}, \ldots, j_{a(i)}\right\} \sqcup\left\{l_{1}, \ldots, l_{b(i)}\right\} \text {, for } 1 \leq i \leq n-k .
$$

If no element of $\left\{j_{1}, \ldots, j_{\bar{k}-k}\right\}$ is $\leq i$, put $a(i)=0$ and omit the first summand and if no element of $\left\{l_{1}, \ldots, l_{n-\bar{k}}\right\}$ is $\leq i$, put $b(i)=0$ and omit the second summand. Note that (3.85) implies

$$
\begin{aligned}
& \{0, \ldots, k, \pi(k+1), \ldots, \pi(k+i)\}= \\
& \left\{0, \ldots, k, \pi\left(k+j_{1}\right), \ldots, \pi\left(k+j_{a(i)}\right)\right\} \cup\left\{\pi\left(k+l_{1}\right), \ldots, \pi\left(k+l_{b(i)}\right)\right\},
\end{aligned}
$$

which yields

$$
\begin{aligned}
& b^{0 \ldots k \pi(k+1) \ldots \pi(k+i)}= \\
& \frac{1}{k+i+1}\left(v_{0}+\ldots+v_{k}+v_{\pi\left(k+j_{1}\right)}+\ldots+v_{\pi\left(k+j_{a(i)}\right)}+\right. \\
& v_{\pi\left(k+l_{1}\right)}+\ldots+v_{\pi\left(k+l_{b(i)}\right)} .
\end{aligned}
$$

Now note that $\pi\left(k+j_{a}\right) \leq \bar{k}$ implies $p_{\bar{\tau} \sigma}\left(v_{\pi\left(k+j_{a}\right)}\right)=v_{\pi\left(k+j_{a}\right)}$ and $\pi\left(k+l_{b}\right) \geq$ $\bar{k}+1$ implies $p_{\bar{\tau} \sigma}\left(v_{\pi\left(k+l_{b}\right)}\right)=b^{\bar{\tau}}$. Therefore, (3.87) yields

$$
\begin{aligned}
& p_{\bar{\tau} \sigma}\left(b^{0 \ldots k \pi(k+1) \ldots \pi(k+i)}\right)= \\
& \frac{1}{k+1+i}\left(v_{0}+\ldots+v_{k}+v_{\pi\left(k+j_{1}\right)}, \ldots,+v_{\pi\left(k+j_{a(i)}\right)}\right)+\frac{b(i)}{k+1+i} b^{\bar{\tau}}= \\
& \frac{k+1+a(i)}{k+1+i} b^{0 \ldots k \pi\left(k+j_{1}\right) \ldots \pi\left(k+j_{a(i)}\right)}+\frac{b(i)}{k+1+i} b^{\bar{\tau}} .
\end{aligned}
$$

Note that $a(i)+b(i)=i$ and thus,

$$
v_{m}=\frac{k+1+a(i)}{k+1+i} v_{m}+\frac{b(i)}{k+1+i} v_{m}, 0 \leq m \leq k .
$$

Summing up (3.88) and (3.89), one obtains

$$
p_{\bar{\tau} \sigma}\left(w_{m}^{0 \ldots k \pi(k+1) \ldots \pi(k+i)}\right)=\frac{k+1+a(i)}{k+1+i} w_{m}^{0 \ldots k \pi\left(k+j_{1}\right) \ldots \pi\left(k+j_{a(i)}\right)}+\frac{b(i)}{k+1+i} w_{m}^{\bar{\tau}} .
$$


Formula (3.90), for $m=0$, shows that the point $p_{\bar{\tau} \sigma}\left(w_{0}^{0 \ldots k \pi(k+1) \ldots \pi(k+i)}\right)$ belongs to the 1 -simplex $\left[w_{0}^{0 \ldots k \pi\left(k+j_{1}\right) \ldots \pi\left(k+j_{a(i)}\right)}, w_{0}^{\bar{\tau}}\right]$. However, this 1-simplex is contained in $d_{\rho}^{\tau}$, because of (3.84) and of the fact that $a(i) \leq i$ implies $j_{a(i)} \leq j_{i}$. This completes the proof of (3.78).

Continuing the proof of assertion $(i)$ of Lemma 3.11, consider the face of $c_{\pi}^{\tau}$, whose vertex scheme consists of the last $n-j_{\bar{k}-k}+1$ rows of the vertex scheme (3.11) for $c_{\pi}^{\tau}$, i.e., it consists of the rows

$$
w_{0}^{0 \ldots k \pi(k+1) \ldots \pi(k+i)} \ldots w_{m}^{0 \ldots k \pi(k+1) \ldots \pi(k+i)} \ldots w_{k}^{0 \ldots k \pi(k+1) \ldots \pi(k+i)},
$$

where $j_{\bar{k}-k} \leq i \leq n-k, 0 \leq m \leq k$. We denote that face of $c_{\pi}^{\tau}$ by $c_{\pi \vartheta}^{\tau \bar{\tau}}$.

Since $a(i)$ is the largest integer $a, 1 \leq a \leq \bar{k}-k$, such that $j_{a} \leq i$, we see that $j_{\bar{k}-k} \leq i$ implies $a(i)=\bar{k}-k$. Therefore,

$$
\begin{aligned}
& \left\{0, \ldots, k, \pi\left(k+j_{1}\right), \ldots, \pi\left(k+j_{a(i)}\right)\right\}= \\
& \left\{0, \ldots, k, \pi\left(k+j_{1}\right), \ldots, \pi\left(k+j_{\bar{k}-k}\right)\right\}= \\
& \{0, \ldots, k, k+1, \ldots, \bar{k}\}=\{0, \ldots, \bar{k}\} .
\end{aligned}
$$

Moreover, (3.83) implies

$$
\left\{\pi\left(k+l_{1}\right), \ldots, \pi\left(k+l_{b(i)}\right)\right\}=\{\vartheta(\bar{k}+1), \ldots, \vartheta(\bar{k}+b(i))\} .
$$

Consequently, (3.86) assumes the form

$$
\begin{aligned}
& \{0, \ldots, k, \pi(k+1), \ldots, \pi(k+i)\}= \\
& \{0, \ldots, \bar{k}, \vartheta(\bar{k}+1), \ldots, \vartheta(\bar{k}+b(i))\}
\end{aligned}
$$

and thus, the row (3.91) coincides with the row

$$
w_{0}^{0 \ldots \bar{k} \vartheta(\bar{k}+1) \ldots \vartheta(\bar{k}+b(i))} \ldots w_{m}^{0 \ldots \bar{k} \vartheta(\bar{k}+1) \ldots \vartheta(\bar{k}+b(i))} \ldots w_{k}^{0 \ldots \bar{k} \vartheta(\bar{k}+1) \ldots \vartheta(\bar{k}+b(i))} .
$$

Let us now extend the vertex scheme of $d_{\pi \vartheta}^{\tau \bar{\tau}}$ with rows written in the form (3.95), by allowing the subscript $m$ to vary between 0 and $\bar{k}$. The new scheme differs from the previous one by $\bar{k}-k$ terminal columns. Clearly, every row of the new scheme is a row of the scheme (3.11) for $c_{\vartheta}^{\bar{\tau}}$, which consists of the initial row $w_{0}^{0 \ldots . \bar{k}}, \ldots, w_{\bar{k}}^{0 \ldots . . \bar{k}}$ (which does not belong to the new scheme) and of the rows

$$
w_{0}^{0 \ldots \bar{k} \vartheta(\bar{k}+1) \ldots \vartheta(\bar{k}+b)} \ldots w_{m}^{0 \ldots \bar{k} \vartheta(\bar{k}+1) \ldots \vartheta(\bar{k}+b)} \ldots w_{k}^{0 \ldots \bar{k} \vartheta(\bar{k}+1) \ldots \vartheta(\bar{k}+b)},
$$

where $1 \leq b \leq n-\bar{k}$ (not every $b$ is of the form $b(i)$ and thus, (3.96) need not be a row of the new scheme). All this shows that $c_{\pi \vartheta}^{\tau \bar{\tau}}$ is a (proper) face of $c_{\vartheta}^{\bar{\tau}}$. implies

To prove (3.79), first note that, for $j_{\bar{k}-k} \leq i \leq n-k, 0 \leq m \leq k,(3.92)$

$$
w_{m}^{0 \ldots k, \pi\left(k+j_{1}\right) \ldots \pi\left(k+j_{a(i)}\right)}=w_{m}^{0 \ldots \bar{k}} .
$$

Using formula (3.90) and the fact that $a(i)+b(i)=i$, one concludes that

$$
p_{\bar{\tau} \sigma}\left(w_{m}^{0 \ldots k \pi(k+1) \ldots \pi(k+i)}\right)=w_{m}^{\bar{\tau}} .
$$


Consequently, $p_{\bar{\tau} \sigma}$ maps the set of vertices of $c_{\pi \vartheta}^{\tau \bar{\tau}}$ onto the set $\left\{w_{0}^{\bar{\tau}}, \ldots, w_{k}^{\bar{\tau}}\right\}$. Since the latter set spans the simplex $\left[w_{0}^{\bar{\tau}}, \ldots, w_{k}^{\bar{\tau}}\right]$, it follows that

$$
p_{\bar{\tau} \sigma}\left(c_{\pi \vartheta}^{\tau \bar{\tau}}\right)=\left[w_{0}^{\bar{\tau}}, \ldots, w_{k}^{\bar{\tau}}\right] .
$$

Now $c_{\pi \vartheta}^{\tau \bar{\tau}} \subseteq c_{\pi}^{\tau}$ implies

$$
c_{\pi \vartheta}^{\tau \bar{\tau}} \subseteq\left(p_{\bar{\tau} \sigma} \mid c_{\pi}^{\tau}\right)^{-1}\left[w_{0}^{\bar{\tau}}, \ldots, w_{k}^{\bar{\tau}}\right] .
$$

To prove the opposite inclusion, it suffices to show that, for every point $u \in c_{\pi}^{\tau} \backslash c_{\pi \vartheta}^{\tau \bar{\tau}}$, one has $p_{\bar{\tau} \sigma}(u) \notin\left[w_{0}^{\bar{\tau}}, \ldots, w_{k}^{\bar{\tau}}\right]$. We will prove this assertion for vertices $w$ of $c_{\pi}^{\tau}$, which do not belong to the face $c_{\pi \vartheta}^{\tau \bar{\tau}}$ of $c_{\pi}^{\tau}$. Then Lemma 2.1 in Subsection 2.1 (with $C=c_{\pi}^{\tau}, C^{\prime}=c_{\rho}^{\tau}, p=p_{\bar{\tau} \sigma} \mid c_{\pi}^{\tau}, D=c_{\pi \vartheta}^{\tau \bar{\tau}}$ and $\left.D^{\prime}=\left[w_{0}^{\bar{\tau}}, \ldots, w_{k}^{\bar{\tau}}\right]\right)$ will yield the desired assertion for points $u \in c_{\pi}^{\tau} \backslash c_{\pi \vartheta}^{\tau \bar{\tau}}$.

To prove that the vertices $w$ of $c_{\pi}^{\tau}$, which do not belong to $c_{\pi \vartheta}^{\tau \bar{\tau}}$, have the required property, it suffices to show that the barycentric coordinate

$$
\lambda_{\rho(\bar{k})}\left(p_{\bar{\tau} \sigma}(w)\right)<\frac{1}{2(\bar{k}+1)},
$$

while for points $y \in\left[w_{0}^{\bar{\tau}}, \ldots, w_{k}^{\bar{\tau}}\right]$, one has

$$
\lambda_{\rho(\bar{k})}(y)=\frac{1}{2(\bar{k}+1)} .
$$

To prove (3.102), it suffices to show that, for the vertices $w_{m}^{\bar{\tau}}, 0 \leq m \leq k$, one has

$$
\lambda_{\rho(\bar{k})}\left(w_{m}^{\bar{\tau}}\right)=\frac{1}{2(\bar{k}+1)} .
$$

Indeed, $\rho(\bar{k}) \in\{k+1, \ldots, \bar{k}\}$ and thus, $\lambda_{\rho(\bar{k})}\left(v_{m}\right)=0$. Since $w_{m}^{\bar{\tau}}=\frac{1}{2}\left(v_{m}+b^{\bar{\tau}}\right)$, we see that $\lambda_{\rho(\bar{k})}\left(w_{m}^{\bar{\tau}}\right)=\frac{1}{2} \lambda_{\rho(\bar{k})}\left(b^{\bar{\tau}}\right)=\frac{1}{2(\bar{k}+1)} \lambda_{\rho(\bar{k})}\left(v_{0}+\ldots+v_{k}+v_{k+1}+\ldots+\right.$ $\left.v_{\bar{k}}\right)=\frac{1}{2(\bar{k}+1)}$.

To prove (3.101), note that either $w$ belongs to the row $w_{0}^{\tau} \ldots w_{m}^{\tau} \ldots w_{k}^{\tau}$ or it belongs to one of the rows (3.91), where $1 \leq i<j_{\bar{k}-k}$. If $w=w_{m}^{\tau}$, then $p_{\bar{\tau} \sigma}\left(w_{m}^{\tau}\right)=w_{m}^{\tau}$ and thus, $\lambda_{\rho(\bar{k})} p_{\bar{\tau} \sigma}\left(w_{m}^{\tau}\right)=\lambda_{\rho(\bar{k})}\left(w_{m}^{\tau}\right)=0$, because $w_{m}^{\tau}=$ $\frac{1}{2} v_{m}+\frac{1}{2(k+1)}\left(v_{0}+\ldots+v_{k}\right)$. Now assume that $w=w_{m}^{0 \ldots k \pi(k+1) \ldots \pi(k+i)}$. Since $j_{a(i)} \leq i$, the assumption $i<j_{\bar{k}-k}$ implies that $j_{a(i)}<j_{\bar{k}-k}$ and thus, $a(i)<$ $\bar{k}-k$. Since $\pi$ is an injection, it follows that $\pi\left(k+j_{\bar{k}-k}\right) \notin\left\{\pi\left(k+j_{1}\right) \ldots, \pi(k+\right.$ $\left.\left.j_{a(i)}\right)\right\}$. By (3.82), $\pi\left(k+j_{\bar{k}-k}\right)=\rho(k+\bar{k}-k)=\rho(\bar{k})$ and thus, $\rho(\bar{k}) \notin$ $\left\{\pi\left(k+j_{1}\right) \ldots, \pi\left(k+j_{a(i)}\right)\right\}$. Since also $\rho(\bar{k}) \notin\{0, \ldots, k\}$, it follows that

$$
\lambda_{\rho(\bar{k})}\left(w_{m}^{0 \ldots k \pi\left(k+j_{1}\right) \ldots, \pi\left(k+j_{a(i)}\right)}\right)=0 .
$$


Therefore, application of $\lambda_{\rho(\bar{k})}$ to (3.90) and (3.103) shows that

$$
\begin{aligned}
& \lambda_{\rho(\bar{k})}\left(p_{\bar{\tau} \sigma}\left(w_{m}^{0 \ldots k \pi(k+1) \ldots \pi(k+i)}\right)\right)= \\
& \frac{b(i)}{k+1+i} \lambda_{\rho(\bar{k})}\left(w_{m}^{\bar{\tau}}\right)=\frac{b(i)}{2(k+1+i)(\bar{k}+1)}<\frac{1}{2(\bar{k}+1)},
\end{aligned}
$$

because $b(i) \leq i$ and $k+1+i>i$.

(ii) Let $c \frac{\overline{\bar{\tau}}}{\bar{\pi}}$ be an $n$-cell from $L(\sigma)$. To prove (3.80), consider an arbitrary point $u \in c_{\bar{\pi}}^{\bar{\tau}}$. It is of the form $u=s+t-w_{0}^{0 \ldots \bar{k}}$, where $s \in c^{\bar{\tau}}$ and $t \in d_{\bar{\pi}}^{\bar{\tau}}$. Recall that $p_{\bar{\tau} \sigma}(s)=s, p_{\bar{\tau} \sigma}\left(w_{0}^{0 \ldots \bar{k}}\right)=w_{0}^{0 \ldots \bar{k}}$ and $p_{\bar{\tau} \sigma}(t)=w_{0}^{0 \ldots . \bar{k}}$ (see (3.51)). Therefore, $p_{\bar{\tau} \sigma}(u)=s+w_{0}^{0 \ldots . \bar{k}}-w_{0}^{0 \ldots \bar{k}}=s \in c^{\bar{\tau}}$ and thus, $p_{\bar{\tau} \sigma}\left(c_{\bar{\pi}}^{\bar{\tau}}\right) \subseteq c^{\bar{\tau}}$. Define $c_{\bar{\pi}}^{\tau \bar{\tau}}$ as the face of $c_{\bar{\pi}}^{\bar{\tau}}$, whose vertex scheme consists of the first $k+1$ columns of the vertex scheme of $c \frac{\bar{\tau}}{\pi}$. Clearly, $c c_{\bar{\pi}}^{\bar{\tau}} \leq c \frac{\bar{\tau}}{\pi}$.

If a point $u$ belongs to the left side of (3.81), then $u \in c_{\bar{\pi}}^{\bar{\tau}}$ and $p_{\bar{\tau} \sigma}(u) \in$ $\left[w_{0}^{\bar{\tau}}, \ldots, w_{k}^{\bar{\tau}}\right]$. Since $u=s+t-w_{0}^{\bar{\tau}}$, where $s \in c^{\bar{\tau}}$ and $t \in d_{\bar{\pi}}^{\bar{\tau}}$, we see that $s=p_{\bar{\tau} \sigma}(u) \in\left[w_{0}^{\bar{\tau}}, \ldots, w_{k}^{\bar{\tau}}\right]$ and therefore, $u$ belongs to the convex polytope spanned by the vertices belonging to the first $k+1$ columns of the vertex scheme of $c_{\frac{\pi}{\pi}}^{\bar{\tau}}$, i.e., $u \in c_{\frac{\tau}{\pi}}^{\bar{\tau}}$. Conversely, if $u$ belongs to the right side of (3.81), then it is of the form $u=s+t-w_{0}^{\bar{\tau}}$, where $s \in\left[w_{0}^{\bar{\tau}}, \ldots, w_{k}^{\bar{\tau}}\right]$ and $t \in d_{\bar{\pi}}$. Consequently, $p_{\bar{\tau} \sigma}(u)=s+w_{0}^{\bar{\tau}}-w_{0}^{\bar{\tau}}=s \in\left[w_{0}^{\bar{\tau}}, \ldots, w_{k}^{\bar{\tau}}\right]$, which shows that also the right side of (3.81) is contained in its left side and thus, equality (3.81) holds.

3.7.3.2 Proof in Case $(c)$. Recall that $\tau^{\prime} \neq \tau^{\prime \prime}$ and $\bar{\tau}=\operatorname{Conv}\left(\tau^{\prime} \cup \tau^{\prime \prime}\right)<\sigma$. If $\tau^{\prime} \cap \tau^{\prime \prime} \neq \emptyset$, then $\tau^{\prime} \cap \tau^{\prime \prime}=\tau$ is a simplex, which is a common face of both $\tau^{\prime}$ and $\tau^{\prime \prime}$. There is no loss of generality in assuming that $\tau=\left[v_{0}, \ldots, v_{k}\right]$ and $\bar{\tau}=\left[v_{0}, \ldots, v_{\bar{k}}\right]$. Clearly, $\tau \leq \tau^{\prime} \leq \bar{\tau}$ and $\tau \leq \tau^{\prime \prime} \leq \bar{\tau}$. We distinguish two cases, either $\tau^{\prime}$ and $\tau^{\prime \prime}$ are incomparable, i.e, $\tau^{\prime} \not \leq \tau^{\prime \prime}$ and $\tau^{\prime \prime} \not \leq \tau^{\prime}$ or they are comparable, i.e., one of the relations $\tau^{\prime} \leq \tau^{\prime \prime}, \tau^{\prime \prime} \leq \tau^{\prime}$ holds. In the first case one cannot have $\tau=\tau^{\prime}$, because $\tau \leq \tau^{\prime \prime}$ would imply $\tau^{\prime} \leq \tau^{\prime \prime}$, contrary to the assumptions that $\tau^{\prime}$ and $\tau^{\prime \prime}$ are incomparable. Consequently, $\tau<\tau^{\prime}$. Similarly, $\tau^{\prime}<\bar{\tau}$, because $\tau^{\prime}=\bar{\tau}$ and $\tau^{\prime \prime} \leq \bar{\tau}$ would imply $\tau^{\prime \prime} \leq \tau^{\prime}$, which also contradicts the assumption that $\tau^{\prime}$ and $\tau^{\prime \prime}$ are incomparable. Consequently, in the first case we must have $\tau<\tau^{\prime}<\bar{\tau}$ and analogously, $\tau<\tau^{\prime \prime}<\bar{\tau}$. In the second case, there is no loss of generality in assuming that $\tau^{\prime} \leq \tau^{\prime \prime}$ and thus, $\tau^{\prime}<\tau^{\prime \prime}$. By the definition of $\tau$ and $\bar{\tau}$, it follows that $\tau=\tau^{\prime}$ and $\bar{\tau}=\tau^{\prime \prime}$. In view of this discussion, the following lemma establishes the desired assertion in Case $(c)$.

LEMMA 3.12.

(i) Let $\tau^{\prime}=\left[v_{0}, \ldots, v_{k^{\prime}}\right], \tau^{\prime \prime}=\left[v_{0}, \ldots, v_{k}, v_{k^{\prime}+1}, \ldots, v_{\bar{k}}\right], \tau^{\prime} \cap \tau^{\prime \prime}=\tau=$ $\left[v_{0}, \ldots, v_{k}\right], 0 \leq k<k^{\prime}<\bar{k}<n$, and let $c_{\pi^{\prime}}^{\tau^{\prime}}, c_{\pi^{\prime \prime}}^{\tau^{\prime \prime}}$ be $n$-cells from $L(\sigma)$. Then the intersection $c_{\pi^{\prime}}^{\tau^{\prime}} \cap c_{\pi^{\prime \prime}}^{\tau^{\prime \prime}}$ is a common face of $c_{\pi^{\prime}}^{\tau^{\prime}}$ and $c_{\pi^{\prime \prime}}^{\tau^{\prime \prime}}$. 
(ii) Let $\tau=\left[v_{0}, \ldots, v_{k}\right], \bar{\tau}=\left[v_{0}, \ldots, v_{\bar{k}}\right], 0 \leq k<\bar{k}<n$, and let $c_{\pi}^{\tau}, c_{\bar{\pi}}^{\bar{\tau}}$ be $n$-cells from $L(\sigma)$. Then the intersection $c_{\pi}^{\tau} \cap c_{\bar{\pi}}^{\bar{\tau}}$ is a common face of $c_{\pi}^{\tau}$ and $c_{\bar{\pi}}^{\bar{\tau}}$.

(iii) Let $\tau^{\prime}=\left[v_{0}, \ldots, v_{k^{\prime}}\right], \tau^{\prime \prime}=\left[v_{k^{\prime}+1}, \ldots, v_{\bar{k}}\right], 0 \leq k^{\prime}<\bar{k}<n$, and let $c_{\pi^{\prime}}^{\tau^{\prime}}, c_{\pi^{\prime \prime}}^{\tau^{\prime \prime}}$ be $n$-cells from $L(\sigma)$. Then the intersection $c_{\pi^{\prime}}^{\tau^{\prime}} \cap c_{\pi^{\prime \prime}}^{\tau^{\prime \prime}}=\emptyset$.

Proof. Case $(i)$. We will first exhibit two permutations $\vartheta^{\prime}, \vartheta^{\prime \prime}$ of the set $\{\bar{k}+1, \ldots, n\}$, a cell $c_{\pi^{\prime} \vartheta^{\prime}}^{\tau^{\prime}}$, which is a common face of $c_{\pi^{\prime}}^{\tau^{\prime}}$ and $c_{\vartheta^{\prime}}^{\bar{\tau}}$ and a cell $c_{\pi^{\prime \prime} \vartheta^{\prime \prime}}^{\tau^{\prime \prime} \bar{\tau}}$, which is a common face of $c_{\pi^{\prime \prime}}^{\tau^{\prime \prime}}$ and $c_{\vartheta^{\prime \prime}}^{\bar{\tau}}$. We will then prove that the intersection $c_{\pi^{\prime} \vartheta^{\prime}}^{\tau^{\prime} \bar{\tau}} \cap c_{\pi^{\prime \prime} \vartheta^{\prime \prime}}^{\tau^{\prime \prime} \bar{\tau}}$ is a common face of the $n$-cells $c_{\pi^{\prime}}^{\tau^{\prime}}$ and $c_{\pi^{\prime \prime}}^{\tau^{\prime \prime}}$. Therefore, the assertion will be proved if we also show that

$$
c_{\pi^{\prime}}^{\tau^{\prime}} \cap c_{\pi^{\prime \prime}}^{\tau^{\prime \prime}}=c_{\pi^{\prime} \vartheta^{\prime}}^{\tau^{\prime} \bar{\tau}} \cap c_{\pi^{\prime \prime} \vartheta^{\prime \prime}}^{\tau^{\prime \prime}} \bar{\tau}
$$

To carry out this program, we first apply Lemma 3.11.(i) to the $n$-cells $c_{\pi^{\prime}}^{\tau^{\prime}}, c_{\pi^{\prime \prime}}^{\tau^{\prime \prime}} \in L(\sigma)$. One obtains a permutation $\rho^{\prime}$ of the set $\left\{k^{\prime}+1, \ldots, \bar{k}\right\}$ and a permutation $\rho^{\prime \prime}$ of the set $\left\{k+1, \ldots, k^{\prime}\right\}$ such that the $n$-cells $c_{\rho^{\prime}}^{\tau^{\prime}}, c_{\rho^{\prime \prime}}^{\tau^{\prime \prime}} \in L(\bar{\tau})$ satisfy the following relations:

$$
\begin{gathered}
p_{\bar{\tau} \sigma}\left(c_{\pi^{\prime}}^{\tau^{\prime}}\right) \subseteq c_{\rho^{\prime}}^{\tau^{\prime}}, \\
p_{\bar{\tau} \sigma}\left(c_{\pi^{\prime \prime}}^{\tau^{\prime \prime}}\right) \subseteq c_{\rho^{\prime \prime}}^{\tau^{\prime \prime}} .
\end{gathered}
$$

One also obtains permutations $\vartheta^{\prime}, \vartheta^{\prime \prime}$ of the set $\{\bar{k}+1, \ldots, n\}$, a common face $c_{\pi^{\prime} \vartheta^{\prime}}^{\tau^{\prime} \bar{\tau}}$ of $c_{\pi^{\prime}}^{\tau^{\prime}}$ and $c_{\vartheta^{\prime}}^{\bar{\tau}}$ and a common face $c_{\pi^{\prime \prime} \vartheta^{\prime \prime}}^{\tau^{\prime \prime}}$ of $c_{\pi^{\prime \prime}}^{\tau^{\prime \prime}}$ and $c_{\vartheta^{\prime \prime}}^{\bar{\tau}}$ such that

$$
\begin{aligned}
& \left(p_{\bar{\tau} \sigma} \mid c_{\pi^{\prime}}^{\tau^{\prime}}\right)^{-1}\left[w_{0}^{\bar{\tau}}, \ldots, w_{k}^{\bar{\tau}}\right]=c_{\pi^{\prime} \vartheta^{\prime}}^{\tau^{\prime} \bar{\tau}}, \\
& \left(p_{\bar{\tau} \sigma} \mid c_{\pi^{\prime \prime}}^{\tau^{\prime \prime}}\right)^{-1}\left[w_{0}^{\bar{\tau}}, \ldots, w_{k}^{\bar{\tau}}\right]=c_{\pi^{\prime \prime} \vartheta^{\prime \prime}}^{\tau^{\prime \prime} \bar{\tau}} .
\end{aligned}
$$

We now apply Lemma 3.9 to the $n$-cells $c_{\vartheta^{\prime}}^{\bar{\tau}}$ and $c_{\vartheta^{\prime \prime}}^{\bar{\tau}}$ and conclude that their intersection $c_{\vartheta^{\prime}}^{\bar{\tau}} \cap c_{\vartheta^{\prime \prime}}^{\bar{\tau}}$ is a common face of both cells $c_{\vartheta^{\prime}}^{\bar{\tau}}$ and $c_{\vartheta^{\prime \prime}}^{\bar{\tau}}$. Since $c_{\pi^{\prime} \vartheta^{\prime}}^{\tau^{\prime} \bar{\tau}} \leq c_{\vartheta^{\prime}}^{\bar{\tau}}$ and $c_{\pi^{\prime \prime} \vartheta^{\prime}}^{\tau^{\prime \prime} \bar{\tau}} \leq c_{\vartheta^{\prime \prime}}^{\bar{\tau}}$, the intersection $c_{\pi^{\prime} \vartheta^{\prime}}^{\tau^{\prime} \bar{\tau}} \cap c_{\pi^{\prime \prime} \vartheta^{\prime \prime}}^{\tau^{\prime \prime} \bar{\tau}}$ is a common face of both cells $c_{\pi^{\prime} \vartheta^{\prime}}^{\tau^{\prime} \bar{\tau}}$ and $c_{\pi^{\prime \prime} \vartheta^{\prime \prime}}^{\tau^{\prime \prime}}$. This is a consequence of the following elementary fact.

Let $C^{\prime}, C^{\prime \prime}$ be convex polytopes in a vector space $V$ and let $D^{\prime} \leq C^{\prime}$ and $D^{\prime \prime} \leq C^{\prime \prime}$ be their faces. If $C^{\prime} \cap C^{\prime \prime}$ is a common face of $C^{\prime}$ and $C^{\prime \prime}$, then $D^{\prime} \cap D^{\prime \prime}$ is a common face of $D^{\prime}$ and $D^{\prime \prime}$.

Since, $c_{\pi^{\prime} \vartheta^{\prime}}^{\tau^{\prime} \bar{\tau}} \leq c_{\pi^{\prime}}^{\tau^{\prime}}$ and $c_{\pi^{\prime \prime} \vartheta^{\prime \prime}}^{\tau^{\prime \prime}} \leq c_{\pi^{\prime \prime}}^{\tau^{\prime \prime}}$, one concludes that $c_{\pi^{\prime} \vartheta^{\prime}}^{\tau^{\prime} \bar{\tau}} \cap c_{\pi^{\prime \prime} \vartheta^{\prime \prime}}^{\tau^{\prime \prime} \bar{\tau}}$ is also a common face of the cells $c_{\pi^{\prime}}^{\tau^{\prime}}$ and $c_{\pi^{\prime \prime}}^{\tau^{\prime \prime}}$.

To complete the proof of Lemma 3.12 in Case (i), it remains to prove (3.106). We will first show that

$$
c_{\pi^{\prime}}^{\tau^{\prime}} \cap c_{\pi^{\prime \prime}}^{\tau^{\prime \prime}}=\left(p_{\bar{\tau} \sigma} \mid\left(c_{\pi^{\prime}}^{\tau^{\prime}} \cap c_{\pi^{\prime \prime}}^{\tau^{\prime \prime}}\right)\right)^{-1}\left(c_{\rho^{\prime}}^{\tau^{\prime}} \cap c_{\rho^{\prime \prime}}^{\tau^{\prime \prime}}\right) .
$$


Indeed, (3.107) and (3.108) imply $p_{\bar{\tau} \sigma}\left(c_{\pi^{\prime}}^{\tau^{\prime}} \cap c_{\pi^{\prime \prime}}^{\tau^{\prime \prime}}\right) \subseteq p_{\bar{\tau} \sigma}\left(c_{\pi^{\prime}}^{\tau^{\prime}}\right) \cap p_{\bar{\tau} \sigma}\left(c_{\pi^{\prime \prime}}^{\tau^{\prime \prime}}\right) \subseteq$ $c_{\rho^{\prime}}^{\tau^{\prime}} \cap c_{\rho^{\prime \prime}}^{\tau^{\prime \prime}}$. Since $p_{\bar{\tau} \sigma}\left(c_{\pi^{\prime}}^{\tau^{\prime}} \cap c_{\pi^{\prime \prime}}^{\tau^{\prime \prime}}\right)=\left(p_{\bar{\tau} \sigma} \mid\left(c_{\pi^{\prime}}^{\tau^{\prime}} \cap c_{\pi^{\prime \prime}}^{\tau^{\prime \prime}}\right)\right)\left(c_{\pi^{\prime}}^{\tau^{\prime}} \cap c_{\pi^{\prime \prime}}^{\tau^{\prime \prime}}\right)$, we see that

$$
c_{\pi^{\prime}}^{\tau^{\prime}} \cap c_{\pi^{\prime \prime}}^{\tau^{\prime \prime}} \subseteq\left(p_{\bar{\tau} \sigma} \mid\left(c_{\pi^{\prime}}^{\tau^{\prime}} \cap c_{\pi^{\prime \prime}}^{\tau^{\prime \prime}}\right)\right)^{-1}\left(c_{\rho^{\prime}}^{\tau^{\prime}} \cap c_{\rho^{\prime \prime}}^{\tau^{\prime \prime}}\right) .
$$

The opposite inclusion also holds, because, for an arbitrary function $f: X \rightarrow$ $Y$ and for arbitrary subsets $A \subseteq X, B \subseteq Y$, one has $(f \mid A)^{-1}(B) \subseteq A$ (by the very definition of the restriction $f \mid A)$.

We now apply to (3.111) the following elementary fact. If $f: X \rightarrow Y$ is a function and $A, A^{\prime} \subseteq X, B \subseteq Y$ are subsets, then $\left(f \mid\left(A^{\prime} \cap A^{\prime \prime}\right)\right)^{-1}(B)=$ $\left(f \mid A^{\prime}\right)^{-1}(B) \cap\left(f \mid A^{\prime \prime}\right)^{-1}(B)$. One obtains the equality

$$
c_{\pi^{\prime}}^{\tau^{\prime}} \cap c_{\pi^{\prime \prime}}^{\tau^{\prime \prime}}=\left(\left(p_{\bar{\tau} \sigma} \mid c_{\pi^{\prime}}^{\tau^{\prime}}\right)^{-1}\left(c_{\rho^{\prime}}^{\tau^{\prime}} \cap c_{\rho^{\prime \prime}}^{\tau^{\prime \prime}}\right)\right) \cap\left(\left(p_{\bar{\tau} \sigma} \mid c_{\pi^{\prime \prime}}^{\tau^{\prime \prime}}\right)-1\left(c_{\rho^{\prime}}^{\tau^{\prime}} \cap c_{\rho^{\prime \prime}}^{\tau^{\prime \prime}}\right)\right) .
$$

Since $\bar{\tau}=\operatorname{Conv}\left(\tau^{\prime} \cup \tau^{\prime \prime}\right)$, Lemma 3.10. $(i)$ is applicable to $c_{\rho^{\prime}}^{\tau^{\prime}}, c_{\rho^{\prime \prime}}^{\tau^{\prime \prime}} \in L(\bar{\tau})$. It implies that

$$
c_{\rho^{\prime}}^{\tau^{\prime}} \cap c_{\rho^{\prime \prime}}^{\tau^{\prime \prime}}=\left[w_{0}^{\bar{\tau}}, \ldots, w_{k}^{\bar{\tau}}\right]
$$

Replacing in (3.113) the expression $c_{\rho^{\prime}}^{\tau^{\prime}} \cap c_{\rho^{\prime \prime}}^{\tau^{\prime \prime}}$ by $\left[w_{0}^{\bar{\tau}}, \ldots, w_{k}^{\bar{\tau}}\right]$ and using (3.109) and (3.110), one obtains the desired equality (3.106).

Case (ii). We will first exhibit a permutation $\vartheta$ of the set $\{\bar{k}+1, \ldots, n\}$, a cell $c_{\pi \vartheta}^{\tau \bar{\tau}}$, which is a common face of $c_{\pi}^{\tau}$ and $c_{\vartheta}^{\bar{\tau}}$ and a cell $c_{\bar{\pi}}^{\tau \bar{\tau}}$, which is a face of $c_{\bar{\pi}}^{\bar{\tau}}$. We will then prove that the intersection $c_{\pi \vartheta}^{\tau \bar{\tau}} \cap c_{\bar{\pi}}^{\tau \bar{\tau}}$ is a common face of the $n$-cells $c_{\pi}^{\tau}$ and $c_{\bar{\pi}}^{\bar{\tau}}$. Therefore, the assertion will be proved if we also show that

$$
c_{\pi}^{\tau} \cap c_{\bar{\pi}}^{\bar{\tau}}=c_{\pi \vartheta}^{\tau \bar{\tau}} \cap c_{\bar{\pi}}^{\tau \bar{\tau}} .
$$

To carry out this program, we first apply Lemma 3.11.(i) to the $n$-cell $c_{\pi}^{\tau} \in$ $L(\sigma)$. One obtains a permutation $\rho$ of the set $\{k+1, \ldots, \bar{k}\}$, a permutation $\vartheta$ of the set $\{\bar{k}+1, \ldots, n\}$ and a cell $c_{\pi \vartheta}^{\tau \bar{\tau}}$, which is a common face of $c_{\pi}^{\tau}$ and $c_{\vartheta}^{\bar{\tau}}$ such that (3.78) and (3.79) hold. Lemma 3.11.(ii), applied to $c_{\frac{\pi}{\pi}}^{\bar{\tau}}$, shows that (3.80) holds and there exists a face $c_{\bar{\pi}}^{\tau \bar{\tau}}$ of $c_{\bar{\pi}}^{\bar{\tau}}$ such that (3.81) holds.

We now apply Lemma 3.9 to the $n$-cells $c_{\vartheta}^{\bar{\tau}}$ and $c_{\bar{\pi}}^{\bar{\tau}}$ and conclude that their intersection $c_{\vartheta}^{\bar{\tau}} \cap c_{\bar{\pi}}^{\bar{\tau}}$ is a common face of $c_{\vartheta}^{\bar{\tau}}$ and $c_{\frac{\pi}{\pi}}^{\bar{\tau}}$. Since $c_{\pi \vartheta}^{\bar{\tau}} \leq c_{\vartheta}^{\bar{\tau}}$ and $c_{\bar{\pi}}^{\tau \bar{\tau}} \leq c_{\bar{\pi}}^{\bar{\tau}}$, it follows that $c_{\pi \vartheta}^{\tau \bar{\tau}} \cap c_{\bar{\pi}}^{\tau \bar{\tau}}$ is a common face of $c_{\pi \vartheta}^{\tau \bar{\tau}}$ and $c_{\bar{\pi}}^{\tau \bar{\tau}}$. However, $c_{\pi \vartheta}^{\tau \bar{\tau}} \leq c_{\pi}^{\tau}, c_{\bar{\pi}}^{\tau \bar{\tau}} \leq c_{\bar{\pi}}^{\bar{\tau}}$ and thus, $c_{\pi \vartheta}^{\tau \bar{\tau}} \cap c_{\bar{\pi}}^{\tau \bar{\tau}}$ is a common face of $c_{\pi}^{\tau}$ and $c_{\bar{\pi}}^{\bar{\tau}}$.

To complete the proof of Lemma 3.12 in Case $(i i)$, it remains to prove (3.115). Using (3.78) and (3.80) and an argument like the one which produced (3.111), one can see that

$$
c_{\pi}^{\tau} \cap c_{\bar{\pi}}^{\bar{\tau}}=\left(p_{\bar{\tau} \sigma} \mid\left(c_{\pi}^{\tau} \cap c_{\bar{\pi}}^{\bar{\tau}}\right)\right)^{-1}\left(c_{\rho}^{\tau} \cap c^{\bar{\tau}}\right) .
$$

The same elementary fact, which we used to derive (3.113) from (3.111), can be applied to (3.116) to obtain

$$
c_{\pi}^{\tau} \cap c_{\bar{\pi}}^{\bar{\tau}}=\left(\left(p_{\bar{\tau} \sigma} \mid c_{\pi}^{\tau}\right)^{-1}\left(c_{\rho}^{\tau} \cap c^{\bar{\tau}}\right)\right) \cap\left(\left(p_{\bar{\tau} \sigma} \mid c_{\bar{\pi}}^{\bar{\tau}}\right)^{-1}\left(c_{\rho}^{\tau} \cap c^{\bar{\tau}}\right)\right) .
$$


Applying Lemma 3.10.(ii) to $c_{\rho}^{\tau} \in L(\bar{\tau})$ and $c^{\bar{\tau}}$, we conclude that

$$
c_{\rho}^{\tau} \cap c^{\bar{\tau}}=\left[w_{0}^{\bar{\tau}}, \ldots, w_{k}^{\bar{\tau}}\right] .
$$

Now replace in (3.117) the expression $c_{\rho}^{\tau} \cap c^{\bar{\tau}}$ by $\left[w_{0}^{\bar{\tau}}, \ldots, w_{k}^{\bar{\tau}}\right]$ and use $(3.79)$ and (3.81). One obtains the desired formula (3.115).

Case (iii). Since $0 \leq k^{\prime}<\bar{k}<n$, we can apply Lemma 3.11.(i) to $c_{\pi^{\prime}}^{\tau^{\prime}} \in L(\sigma)$ and obtain a permutation $\rho^{\prime}$ of the set $\left\{k^{\prime}+1, \ldots, \bar{k}\right\}$ such that the $\bar{k}$-cell $c_{\rho^{\prime}}^{\tau^{\prime}} \in L(\bar{\tau})$ satisfies relation (3.107). Analogously, there is a permutation $\rho^{\prime \prime}$ of the complement $\left\{0, \ldots, k^{\prime}\right\}=\{0, \ldots, \bar{k}\} \backslash\left\{k^{\prime}+1, \ldots, \bar{k}\right\}$ such that the $\bar{k}$-cell $c_{\rho^{\prime \prime}}^{\tau^{\prime \prime}} \in L(\bar{\tau})$ satisfies relation (3.108). Since $\bar{\tau}=\operatorname{Conv}\left(\tau^{\prime} \cup \tau^{\prime \prime}\right)$, Lemma 3.10. $($ iii $)$ is applicable to $c_{\rho^{\prime}}^{\tau^{\prime}}, c_{\rho^{\prime \prime}}^{\tau^{\prime \prime}} \in L(\bar{\tau})(\bar{\tau}$ plays the role of $\sigma)$. Since $\tau^{\prime} \cap \tau^{\prime \prime}=$ $\emptyset$, it shows that $c_{\rho^{\prime}}^{\tau^{\prime}} \cap c_{\rho^{\prime \prime}}^{\tau^{\prime \prime}}=\emptyset$. Therefore, (3.107) and (3.108) yield the desired conclusion that also $c_{\pi^{\prime}}^{\tau^{\prime}} \cap c_{\pi^{\prime \prime}}^{\tau^{\prime \prime}}=\emptyset$.

3.8. To complete the proof that $L(\sigma)$ is a cellular subdivision of $\sigma$, it only remains to establish the following lemma.

Lemma 3.13. If $\sigma \in K$ is $n$-dimensional, then the union of all $n$-cells from $L(\sigma)$ equals $\sigma$.

Proof. It suffices to prove that the $n$-cells from $L(\sigma)$ cover the $n$ simplices of the barycentric subdivision $\sigma^{\prime}$ of $\sigma$. Let $\iota_{n}, \iota_{n-1 n}, \ldots, \iota_{1} \ldots n$ denote the identity permutations of the sets $\{n\},\{n-1, n\}, \ldots,\{1, \ldots, n\}$, respectively. We will prove that the $n$-cells $c^{0 \ldots n}, c_{\iota_{n}}^{0 \ldots n-1}, c_{\iota_{n-1 n}}^{0 \ldots n-2}, \ldots, c_{\iota_{1}, n}^{0} \in L(\sigma)$ cover the $n$-simplex $\left[b^{0}, b^{01}, \ldots, b^{0 \ldots n}\right] \in \sigma^{\prime}$. The assertion for any other $n$-simplex from $\sigma^{\prime}$ is obtained by relabelling the vertices of $\sigma$.

Let $u$ be a point from $\left[b^{0}, \ldots, b^{0 \ldots n}\right]$. We must show that $u$ belongs to at least one of the cells $c^{0 \ldots n}, c_{\iota_{n}}^{0 \ldots n-1}, c_{\iota_{n-1 n}}^{0 \ldots n-2}, \ldots, c_{\iota_{1 \ldots n}}^{0}$. Obviously, this is true if $u \in c^{0 \ldots n}$. Therefore, we will assume that $u \in\left[b^{0}, \ldots, b^{0 \ldots n}\right] \backslash c^{0 \ldots n}$. Let $p_{i}:\left[b^{0}, \ldots, b^{0 \ldots n}\right] \rightarrow\left[b^{0}, \ldots, b^{0 \ldots i}\right], 0 \leq i \leq n$, be the simplicial mapping determined by $p_{i}\left(b^{0 \ldots j}\right)=b^{0 \ldots j}$, for $0 \leq j \leq i$, and $p_{i}\left(b^{0 \ldots j}\right)=b^{0 \cdots i}$, for $i \leq j \leq n$. For $u \in\left[b^{0}, b^{01}, \ldots, b^{0 \ldots n}\right]$, put $u_{i}=p_{i}(u)$ and note that $u_{0}=$ $b^{0}=v_{0}$ and $u_{n}=u$. Also note that $u=\mu_{0} b^{0}+\ldots+\mu_{n} b^{0 \ldots n}, \mu_{0}, \ldots, \mu_{n} \geq 0$, $\mu_{0}+\ldots+\mu_{n}=1$ imply

$$
u_{i}=\mu_{0} b^{0}+\ldots+\mu_{i-1} b^{0 \ldots i-1}+\left(\mu_{i}+\ldots+\mu_{n}\right) b^{0 \ldots i} .
$$

Consequently,

$$
u_{i}-u_{i-1}=\left(\mu_{i}+\ldots+\mu_{n}\right)\left(b^{0 \ldots i}-b^{0 \ldots i-1}\right), 1 \leq i \leq n .
$$

Now note that (3.2) yields

$$
w_{0}^{0 \ldots i}-w_{0}^{0 \ldots i-1}=\frac{1}{2}\left(b^{0 \ldots i}-b^{0 \ldots i-1}\right), 1 \leq i \leq n,
$$


and thus,

$$
u_{i}-u_{i-1}=2\left(\mu_{i}+\ldots+\mu_{n}\right)\left(w_{0}^{0 \ldots i}-w_{0}^{0 \ldots i-1}\right), 1 \leq i \leq n .
$$

If for some integer $k, 0 \leq k \leq n-1$, we sum the relations (3.122), for $k+1 \leq i \leq n$, we obtain

$$
u-u_{k}=t-w_{0}^{0 \ldots k}
$$

where

$$
\begin{aligned}
t-w_{0}^{0 \ldots k}= & 2\left(\mu_{k+1}+\ldots+\mu_{n}\right)\left(w_{0}^{0 \ldots k+1}-w_{0}^{0 \ldots k}\right)+ \\
& 2\left(\mu_{k+2}+\ldots+\mu_{n}\right)\left(w_{0}^{0 \ldots k+2}-w_{0}^{0 \ldots k+1}\right)+\ldots+ \\
& 2 \mu_{n}\left(w_{0}^{0 \ldots n}-w_{0}^{0 \ldots n-1}\right) .
\end{aligned}
$$

We now choose as $k$ the largest integer, $0 \leq k \leq n-1$, such that $u_{k} \in c^{0 \ldots k}$. It exists because $u_{0}=v_{0}=w_{0}^{0}, c^{0}=\left[w_{0}^{0}\right]$ and $u=u_{n} \notin c^{0 \ldots n}$. For that $k$ we will prove that $t \in d_{\iota_{k+1 \ldots n}}^{0 \ldots k}=\left[w_{0}^{0 \ldots k}, \ldots, w_{0}^{0 \ldots n}\right]$ and thus, (3.123) will imply that $u \in c^{0 \ldots k}+d_{\iota_{k+1 \ldots n}}^{0 \ldots k}-w_{0}^{0 \ldots k}=c_{\iota_{k+1 \ldots n}}^{0 \ldots k}$. In the proof we will use the following elementary fact.

If $\left[a_{0}, \ldots, a_{m}\right]$ is an m-simplex, $a-a_{0}=\nu_{1}\left(a_{1}-a_{0}\right)+\nu_{2}\left(a_{2}-a_{1}\right)+\ldots+$ $\nu_{n}\left(a_{n}-a_{n-1}\right)$ and $1 \geq \nu_{1} \geq \nu_{2} \geq \ldots \geq \nu_{m} \geq 0$, then $a \in\left[a_{0}, \ldots, a_{n}\right]$.

Since $2\left(\mu_{k+1}+\ldots+\mu_{n}\right) \geq 2\left(\mu_{k+2}+\ldots+\mu_{n}\right) \geq \ldots \geq 2 \mu_{n} \geq 0$, our assertion concerning $t$ will be proved if we show that

$$
\frac{1}{2} \geq \mu_{k+1}+\ldots+\mu_{n} .
$$

However, by (3.119),

$$
u_{k+1}=\mu_{0} b^{0}+\ldots+\mu_{k} b^{0 \ldots k}+\left(\mu_{k+1}+\ldots+\mu_{n}\right) b^{0 \ldots k+1}
$$

and we see that $\mu_{k+1}+\ldots+\mu_{n}$ is the last barycentric coordinate of $u_{k+1}$ in (3.126). Since $u_{k+1} \in\left[b^{0}, \ldots, b^{0 \ldots k+1}\right] \backslash c^{0 \ldots k+1}$, we see that $(3.125)$ is a consequence of the following assertion. If $u \in\left[b^{0}, \ldots, b^{0 \ldots m}\right] \backslash c^{0 \ldots m}, 0 \leq m \leq n$, and $\beta_{0}, \ldots, \beta_{m}$ are the barycentric coordinates of $u$ with respect to $b^{0}, \ldots, b^{0 \ldots m}$, then $\beta_{m}<\frac{1}{2}$. Indeed, it is well known that a point $u \in \sigma$ belongs to $\left[b^{0}, \ldots, b^{0 \ldots m}\right]$ if and only if its barycentric coordinates $\lambda_{0}, \ldots, \lambda_{m}$ with respect to $v_{0}, \ldots, v_{m}$ satisfy the inequality $\lambda_{0} \geq \ldots \geq \lambda_{m}$. One cannot have $\lambda_{m} \geq \frac{1}{2(m+1)}$, because that would imply that all coordinates $\lambda_{i} \geq \frac{1}{2(m+1)}$, $0 \leq i \leq m$, and thus, $u \in c^{0 \ldots m}$, which contradicts our assumption that $u \notin c^{0 \ldots m}$. Consequently, $\lambda_{m}<\frac{1}{2(m+1)}$. Now, if in $u=\lambda_{0} v_{0}+\ldots+\lambda_{m} v_{m}=$ $\beta_{0} b^{0}+\ldots+\beta_{n} b^{0 \ldots m}$ we substitute the values of $b^{0}, \ldots, b^{0 \ldots m}$ from (3.1), we readily see that $\lambda_{m}=\frac{\beta_{m}}{(m+1)}$ and the desired assertion $\beta_{m}<\frac{1}{2}$ follows. 
3.9. The next lemma will complete the proof of Theorem 3.6.

LEMMA 3.14. If $\sigma \in K$ and $\zeta<\sigma$ is a proper face of $\sigma$, then $L(\zeta) \subseteq L(\sigma)$ and (3.17) holds. For any cell $c \in L(\sigma)$, the intersection $\zeta \cap c$ is a face of $c$, which belongs to $L(\zeta)$. The union $L=\cup_{\sigma \in K} L(\sigma)$ is a cellular subdivision of $K$.

Proof. Let $\zeta<\sigma, \operatorname{dim} \sigma=n$ and $\operatorname{dim} \zeta=m<n$. To prove that $L(\zeta) \subseteq L(\sigma)$, it suffices to prove the assertion that $c \in L(\zeta)$ implies $c \in L(\sigma)$ in the special case, when $c \in L(\zeta)$ is an $m$-cell. Indeed, an arbitrary cell $c \in L(\zeta)$ is a face of an $m$-cell $c^{\prime} \in L(\zeta)$ and the special case of the assertion shows that $c^{\prime} \in L(\sigma)$. Now $c \leq c^{\prime}$ implies that also $c \in L(\sigma)$.

Let us now assume that $\sigma=\left[v_{0}, \ldots, v_{n}\right], \zeta=\left[v_{0}, \ldots, v_{m}\right], 0 \leq m<n$. If $c$ is the central $m$-simplex of $L(\zeta)$, consider the peripheral $n$-cell $c^{\prime}=c_{\iota}^{\zeta} \in L(\sigma)$, where $\iota$ is the identity permutation of the set $\{m+1, \ldots, n\}$. By Lemma 3.2 , the first row in the vertex scheme (3.11) of $c^{\prime}$ consists of the vertices $w_{0}^{0 \ldots m}, \ldots, w_{m}^{0 \ldots m}$ and thus, $\left[w_{0}^{0 \ldots m}, \ldots, w_{m}^{0 \ldots m}\right]$ is a face of $c^{\prime}$. However, $c=$ $\left[w_{0}^{0 \ldots m}, \ldots, w_{m}^{0 \ldots m}\right]<\left[w_{0}^{0 \ldots m}, \ldots, w_{n}^{0 \ldots m}\right]<c^{\prime}$. Since $c^{\prime} \in L(\sigma)$ and $c<c^{\prime}$, it follows that also $c \in L(\sigma)$.

Now let $c=c_{\pi}^{\tau}$ be a peripheral $m$-cell from $L(\zeta)$. There is no loss of generality in assuming that $\tau=\left[v_{0}, \ldots, v_{k}\right], 0 \leq k<m$, and thus, $\pi$ is a permutation of the set $\{k+1, \ldots, m\}$. The vertex scheme (3.11) of $c$ consists of its first row $w_{0}^{0 \ldots k} \ldots w_{k}^{0 \ldots k}$ and of the rows $w_{0}^{0 \ldots k \pi(k+1) \ldots \pi(k+i)} \ldots w_{k}^{0 \ldots k \pi(k+1) \ldots \pi(k+i)}$, where $1 \leq i \leq m-k$. Let $\pi^{\prime}$ be the permutation of the set $\{k+1, \ldots, n\}$, which on $\{k+1, \ldots, m\}$ coincides with $\pi$ and on $\{m+1, \ldots, n\}$ is the identity. The vertex scheme of $c^{\prime}=c_{\pi^{\prime}}^{\tau} \in L(\sigma)$ consists of the row $w_{0}^{0 \ldots k} \ldots w_{k}^{0 \ldots k}$ and of the rows $w_{0}^{0 \ldots k \pi(k+1) \ldots \pi(k+i)} \ldots w_{k}^{0 \ldots k \pi(k+1) \ldots \pi(k+i)}$, where $1 \leq i \leq n-k$. Consequently, the vertex scheme of $c$ coincides with the initial $m-k+1$ rows of the vertex scheme of $c^{\prime}$ and thus, $c \leq c^{\prime}$, which together with $c^{\prime} \in L(\sigma)$ yields the desired conclusion that $c \in L(\bar{\sigma})$.

Note that $L(\zeta) \subseteq L(\sigma)$ implies

$$
L(\zeta) \subseteq \zeta \cap L(\sigma) .
$$

Indeed, if $c \in L(\zeta)$, then $c \subseteq \zeta$ and thus, $c=\zeta \cap c$. However, $c \in L(\zeta)$ implies $c \in L(\sigma)$ and thus, $c \in \zeta \cap L(\sigma)$.

We will now show that, for a simplex $\sigma \in K$, for a proper face $\zeta$ of $\sigma$ and a cell $c \in L(\sigma)$, the intersection $\zeta \cap c$ is a face of $c$, which belongs to $L(\zeta)$. It suffices to prove the assertion in the special case when $\operatorname{dim} \sigma-\operatorname{dim} \zeta=1$. Indeed, if $\operatorname{dim} \sigma-\operatorname{dim} \zeta=k>1$, we consider a sequence of faces $\zeta=\zeta_{k}<$ $\ldots<\zeta_{1}<\sigma$, where the dimensions of consecutive members differ by 1 . The assertion in the special case shows that $\zeta_{1} \cap c \leq c$ and $\zeta_{1} \cap c \in L\left(\zeta_{1}\right)$. Therefore, $\zeta_{2} \cap c=\zeta_{2} \cap \zeta_{1} \cap c \leq \zeta_{1} \cap c \leq c$ and $\zeta_{2} \cap c \in L\left(\zeta_{2}\right)$. Repeating this argument $k$ times, we obtain the desired conclusion that $\zeta \cap c \leq c$ and $\zeta \cap c \in L(\zeta)$. 
We will now show that, for $\operatorname{dim} \sigma=n$, for a face $\zeta$ of $\sigma$ of dimension $\operatorname{dim} \zeta=n-1$ and for $c \in L(\sigma)$, the intersection $\zeta \cap c$ is a face of $c$, which belongs to $L(\zeta)$. It suffices to prove the assertion in the special case when $\operatorname{dim} c=n$. Indeed, an arbitrary cell $c$ from $L(\sigma)$ is a face of an $n$-cell $c^{\prime} \in L(\sigma)$. By the special case, $\zeta \cap c^{\prime}$ is a face of $c^{\prime}$, which belongs to $L(\zeta) \subseteq L(\sigma)$. Note that $c=c^{\prime} \cap c$ and thus, $\zeta \cap c=\left(\zeta \cap c^{\prime}\right) \cap c$. Since $\zeta \cap c^{\prime}$ and $c$ are faces of $c^{\prime} \in L(\sigma)$, it follows that their intersection $\zeta \cap c=\left(\zeta \cap c^{\prime}\right) \cap c$ is a face of $\zeta \cap c^{\prime}$ and $c$. Now $\zeta \cap c \leq \zeta \cap c^{\prime}$ and $\zeta \cap c^{\prime} \in L(\zeta)$ imply that also $\zeta \cap c \in L(\zeta)$.

Let us now assume that $\sigma=\left[v_{0}, \ldots, v_{n}\right], 0 \leq l \leq n$ and $\zeta$ is the $(n-1)$ dimensional face of $\sigma$, which does not contain the vertex $v_{l}$, i.e., $\zeta=S \cap \sigma$, where $S$ is the $(n-1)$-dimensional affine manifold $S=\left\{u \in \operatorname{Aff}(\sigma) \mid \lambda_{l}(u)=0\right\}$. Let $c$ be an $n$-cell of $L(\sigma)$. If $c$ is the central $n$-simplex $c^{\sigma}$, then $c$ lies in the interior of $\sigma$. Since $\zeta \subseteq \partial \sigma$, it follows that $\zeta \cap c=\emptyset$. However, the empty set is a face of $c$ and it belongs to $L(\zeta)$. We will now assume that $c=c_{\pi}^{\tau}$ is a peripheral $n$-cell of $L(\sigma)$. There is no loss of generality in assuming that $\tau=\left[v_{0}, \ldots, v_{k}\right]$, where $0 \leq k<n$ and thus, $\pi$ is a permutation of the set $\{k+$ $1, \ldots, n\}$. We first consider the case when $0 \leq l \leq k$. It is readily seen that, for $0 \leq m \leq k$, one has $\lambda_{l}\left(w_{m}^{0 \ldots k}\right) \geq \frac{1}{2} \lambda_{l}\left(b^{0 \ldots k}\right)=\frac{1}{2(k+1)}>0$. Similarly, for $1 \leq i \leq n-k, \lambda_{l}\left(w_{m}^{0 \ldots k \pi(k+1) \ldots \pi(k+i)}\right) \geq \frac{1}{2} \lambda_{l}\left(b^{0 \ldots k \pi(k+1) \ldots \pi(k+i)}\right)=\frac{1}{2(k+i+1)}>$ 0 . In view of Lemma 3.2, this means that all the vertices of $c$ belong to the convex set $\left\{u \in \operatorname{Aff}(\sigma) \mid \lambda_{l}(u)>0\right\}$ and thus, $c$ is contained in that set. Consequently, $\zeta \cap c=\emptyset$ and the assertion holds.

Now assume that $k+1 \leq l \leq n$. We will show that $c \cap \zeta$ is a face of $c$, which belongs to $L(\zeta)$. In addition, we will show that it is of the form $c \cap \zeta=c^{\tau} \oplus d^{\prime}=c^{\tau}+d^{\prime}-w_{0}^{\tau}$, where $d^{\prime}$ is a face of $d_{\pi}^{\tau}$, which contains the vertex $w_{0}^{\tau}$. First note that $c \subseteq \sigma$ implies $c=\sigma \cap c$ and thus, $\zeta \cap c=S \cap c$. Since $l \geq k+1, \lambda_{l}\left(v_{m}\right)=0$, for $0 \leq m \leq k$, and thus, $\lambda_{l}\left(w_{m}^{0 \ldots k}\right)=\frac{1}{2} \lambda_{l}\left(b^{0 \ldots k}\right)=0$. This shows that the vertices $w_{0}^{0 \ldots k}, \ldots, w_{k}^{0 \ldots k}$ belong to $c$ and $S$ and thus, $S \cap c \neq \emptyset$. Moreover, since $c \subseteq \sigma, c$ is contained in the affine halfspace $S^{+}=\left\{u \in \operatorname{Aff}(\sigma) \mid \lambda_{l}(u) \geq 0\right\}$, which shows that $S$ is a supporting hyperplane, for the $n$-cell $c=c_{\pi}^{\tau} \in L(\sigma)$. It follows that $\zeta \cap c=S \cap c$ is the face of $c$, whose vertices are all the vertices $w$ of $c$, which are contained in $S$, i.e., for which $\lambda_{l}(w)=0$. Note that there is a unique integer $j \in\{1, \ldots, n-k\}$ such that $l=\pi(k+j)$. Clearly, $\lambda_{l}\left(w_{m}^{0 \ldots k \pi(k+1) \ldots \pi(k+i)}\right)=0$, for $1 \leq i<j$, and $\lambda_{l}\left(w_{m}^{0 \ldots k \pi(k+1) \ldots \pi(k+i)}\right)=\frac{1}{2} \lambda_{l}\left(b^{0 \ldots k \pi(k+1) \ldots \pi(k+i)}\right)=\frac{1}{2(k+1+i)}>0$, for $j \leq i \leq n-k$. This shows that $\zeta \cap c$ is the face of $c$, whose vertices are the points belonging to the first $j$ rows of $(3.11)$, i.e., the points $w_{0}^{0 \ldots k}, \ldots, w_{k}^{0 \ldots k}$ and the points $w_{0}^{0 \ldots k \pi(k+1) \ldots \pi(k+i)}, \ldots, w_{k}^{0 \ldots k \pi(k+1) \ldots \pi(k+i)}$, where $1 \leq i<j$. In other words, $\zeta \cap c=c^{\tau}+d^{\prime}-w_{0}^{\tau}$, where $d^{\prime}=\left[w_{0}^{0 \ldots k}, \ldots, w_{0}^{0 \ldots k \pi(k+1) \ldots \pi(k+j-1)}\right] \leq$ $d_{\pi}^{\tau}$. Clearly, the simplex $\eta=\left[v_{0}, \ldots, v_{k}, v_{\pi(k+1)}, \ldots, v_{\pi(k+j-1)}\right]$ is a face of $\zeta$, because its vertices differ from $v_{l}$. Consider the identity permutation $\iota$ of 
the set $\{\pi(k+1), \ldots, \pi(k+j-1)\}$ and note that, by Lemma 3.2, the vertex scheme of the cell $c_{\iota}^{\tau} \in L(\eta)$ coincides with the above described vertex scheme of $\zeta \cap c$ and thus, $\zeta \cap c=c_{\iota}^{\tau}$. However, $c_{\iota}^{\tau} \in L(\eta)$. Since $L(\eta) \subseteq L(\zeta)$, it follows that $\zeta \cap c \in L(\zeta)$. Note that $\operatorname{dim} c_{\iota}^{\tau}=k+j-1=\operatorname{dim} \eta$.

The statement which we just proved implies that

$$
L(\zeta) \supseteq \zeta \cap L(\sigma),
$$

because $c \in L(\sigma)$ implies $\zeta \cap c \in L(\zeta)$. Note that (3.127) and (3.128) yield formula (3.17). The final assertion of Theorem 3.6 that the union $L=\cup_{\sigma \in K} L(\sigma)$ is a cellular subdivision of $K$ is an immediate consequence of (3.17).

The following corollary is an easy consequence of Theorem 3.6.

Corollary 3.15. Let $c, c^{\prime}$ be cells from $L(\sigma)$ and let $W(c), W\left(c^{\prime}\right)$ be sets of their respective vertices. Then $c \cap c^{\prime}$ is the convex hull of the set $W(c) \cap W\left(c^{\prime}\right)$.

Proof. Since $W(c) \subseteq c, W\left(c^{\prime}\right) \subseteq c^{\prime}$ and $c \cap c^{\prime}$ is convex, it follows that Conv $\left(W(c) \cap W\left(c^{\prime}\right)\right) \subseteq c \cap c^{\prime}$. Conversely, let $w$ be a vertex of $c \cap c^{\prime}$. By Theorem 3.6, $c \cap c^{\prime} \leq c$ and $c \cap c^{\prime} \leq c^{\prime}$ and thus, $w$ is a vertex of $c$ and a vertex of $c^{\prime}$. Consequently, $w \in W(c) \cap W\left(c^{\prime}\right) \subseteq \operatorname{Conv}\left(W(c) \cap W\left(c^{\prime}\right)\right)$. Since $c \cap c^{\prime}$ is the convex hull of the set of all of its vertices $w$, it follows that $c \cap c^{\prime} \subseteq \operatorname{Conv}\left(W(c) \cap W\left(c^{\prime}\right)\right)$.

REMARK 3.16. The $n$-cells $c_{\pi}^{\tau}=c^{\tau} \oplus d_{\pi}^{\tau}$ of $L(\sigma)$ depend on the ordering of the vertices of $\sigma=\left[v_{0}, \ldots, v_{n}\right]$, but the subdivision $L(\sigma)$ itself is independent of that ordering. More precisely, $c^{\tau}$ does not depend on the ordering of the vertices of $\tau$, because, for $\tau=\left[v_{0}, \ldots, v_{k}\right]$, one has $c^{\tau}=\left[w_{0}^{\tau}, \ldots, w_{k}^{\tau}\right]$. However, $d_{\pi}^{\tau}$ does depend on the ordering of the remaining vertices of $\sigma$. Indeed, write $\sigma$ in the form $\sigma=\left[v_{0}, \ldots, v_{k}, v_{\pi^{\prime}(k+1)}, \ldots, v_{\pi^{\prime}(n)}\right]$, where $\pi^{\prime}$ is a permutation of $\{k+1, \ldots, n\}$. Consider a permutation $\eta$ of $\{k+1, \ldots, n\}$ and the simplex $d_{\eta}^{\tau}$, obtained using the new ordering of the vertices of $\sigma$. Putting $v_{0}^{\prime}=v_{0}, \ldots, v_{k}^{\prime}=v_{k}, v_{k+1}^{\prime}=v_{\pi^{\prime}(k+1)}, \ldots, v_{n}^{\prime}=v_{\pi^{\prime}(n)}$, the new ordering of $\sigma$ assumes the form $\sigma=\left[v^{\prime}{ }_{0}, \ldots, v_{k}^{\prime}, v^{\prime}{ }_{k+1}, \ldots, v_{n}^{\prime}\right]$ and thus, $d_{\eta}^{\tau}=\left[{w^{\prime}}_{0}^{0 \ldots k}, w_{0}^{\prime 0 \ldots k \eta(k+1)}, \ldots, w_{0}^{\prime 0 \ldots n}\right]$, where ${w^{\prime 0}}_{0}^{0 \ldots k}=\frac{1}{2} v_{0}^{\prime}+$ $\frac{1}{2(k+1)}\left(v_{0}^{\prime}+\ldots+v_{k}^{\prime}\right)=\frac{1}{2} v_{0}+\frac{1}{2(k+1)}\left(v_{0}+\ldots+v_{k}\right)=w_{0}^{0 \ldots k}, w_{0}^{\prime 0 \ldots k \eta(k+1)}=$ $\frac{1}{2} v^{\prime}{ }_{0}+\frac{1}{2(k+2)}\left(v_{0}^{\prime}+\ldots+v^{\prime}{ }_{k}+v^{\prime}{ }_{\eta(k+1)}\right)=\frac{1}{2} v_{0}+\frac{1}{2(k+2)}\left(v_{0}+\ldots+v_{k}+v_{\pi^{\prime} \eta(k+1)}\right)=$ $w_{0}^{0 \ldots k \pi^{\prime} \eta(k+1)}$, etc., ${w_{0}^{\prime}}_{0}^{0 \ldots n}=\frac{1}{2} v_{0}+\frac{1}{2(n+1)}\left(v_{0}+\ldots+v_{n}\right)=w_{0}^{0 \ldots n}$. Consequently, $d_{\eta}^{\tau}=\left[w_{0}^{0 \ldots k}, w_{0}^{0 \ldots k \pi^{\prime} \eta(k+1)}, \ldots, w_{0}^{0 \ldots n}\right]$. However, this simplex has the form of $d_{\pi}^{\tau}$, when one uses the original ordering $v_{0}, \ldots, v_{n}$ of the vertices of $\sigma$ and the permutation $\pi=\pi^{\prime} \eta$. When $\eta$ ranges over the set of all permutations of $\{k+1, \ldots, n\}, \pi=\pi^{\prime} \eta$ will also range over the set of all permutations of $\{k+1, \ldots, n\}$ and thus, the set of all $d_{\eta}^{\tau}$ and the set of all $d_{\pi}^{\tau}$ coincide. It 
follows that also the set of all $c_{\eta}^{\tau}=c^{\tau} \oplus d_{\eta}^{\tau}$ coincides with the set $L(\sigma)$ of all $c_{\pi}^{\tau}=c^{\tau} \oplus d_{\pi}^{\tau}$.

\section{The SECOND CANONICAL SUbDivision $N(K)$}

4.1. We will first define the cellular complex $N(\sigma)$, for ordered $n$ simplices $\sigma=\left[v_{0}, \ldots, v_{n}\right]$, contained in the vector space $V$. By definition, $N(\sigma)$ consists of $n+1 n$-dimensional convex polytopes $c_{v_{i}}^{\sigma}, 0 \leq i \leq n$, and of their faces. Most of the time there will be no danger of misunderstanding if we simplify the notation $c_{v_{i}}^{\sigma}$ to $c_{i}^{\sigma}$. To define $c_{i}^{\sigma}$, one considers the homothetic transformation $\chi_{i}^{\sigma}: \sigma \rightarrow \sigma$ with center $v_{i}$ and ratio $\frac{1}{2}$, i.e., one puts $\chi_{i}^{\sigma}(v)-v_{i}=\frac{1}{2}\left(v-v_{i}\right)$, for $v \in \sigma$. Then $c_{i}^{\prime \sigma}=\chi_{i}^{\sigma}\left[v_{0}, \ldots, v_{i}\right]$ is an $i$-dimensional simplex, which lies in $\left[v_{0}, \ldots, v_{i}\right] \subseteq \operatorname{Aff}\left[v_{0}, \ldots, v_{i}\right]$ and $c_{i}^{\prime \prime \sigma}=\chi_{i}^{\sigma}\left[v_{i}, \ldots, v_{n}\right]$ is an $(n-i)$-dimensional simplex, which lies in $\left[v_{i}, \ldots, v_{n}\right] \subseteq \operatorname{Aff}\left[v_{i}, \ldots, v_{n}\right]$. The intersection Aff $\left[v_{0}, \ldots, v_{i}\right] \cap \operatorname{Aff}\left[v_{i}, \ldots, v_{n}\right]=\left\{v_{i}\right\}$. Therefore, the direct sum $c_{i}^{\prime \sigma} \oplus c_{i}^{\prime \prime}$ is well defined and has $v_{i}$ for its reference point. By definition,

$$
c_{i}^{\sigma}=c_{i}^{\prime \sigma} \oplus c_{i}^{\prime \prime \sigma} \text {. }
$$

Note that $c_{i}^{\sigma}$ is an $n$-dimensional convex polytope contained in $\operatorname{Aff}(\sigma)$. Moreover, every point $u \in c_{i}^{\sigma}$ admits unique points $u^{\prime} \in c_{i}^{\prime \sigma}$ and $u^{\prime \prime} \in c_{i}^{\prime \prime \sigma}$ such that $u=u^{\prime}+u^{\prime \prime}-v_{i}$.
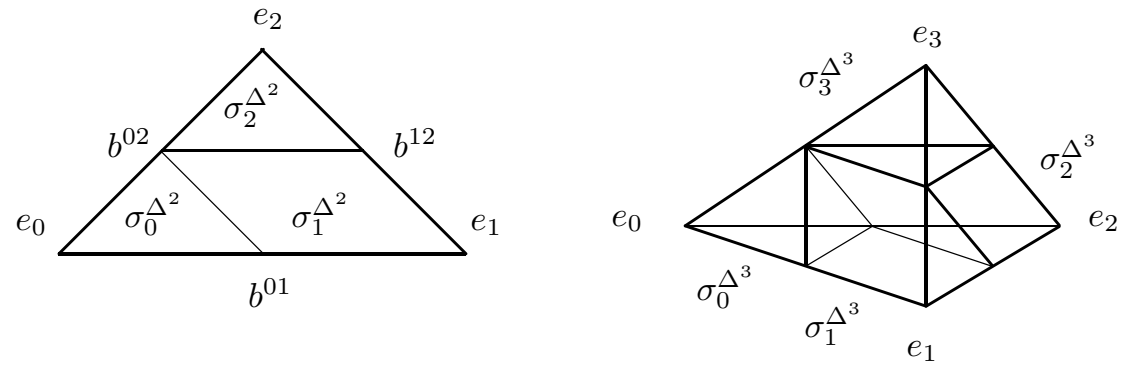

Figure 3. The decompositions $N\left(\Delta^{2}\right)$ and $N\left(\Delta^{3}\right)$

The following lemma describes the vertices and the faces of the $n$-cell $c_{i}^{\sigma}$, when $\sigma=\left[v_{0}, \ldots, v_{n}\right]$. Recall that $b^{i j}$ is the short notation for $b^{\left[v_{i}, v_{j}\right]}=$ $\frac{1}{2}\left(v_{i}+v_{j}\right)($ see $(3.1))$. Note that $b^{i i}=v_{i}$.

LEMMA 4.1. If $\sigma=\left[v_{0}, \ldots, v_{n}\right]$ is an ordered simplex and $0 \leq i \leq n$, then the vertices of the $n$-cell $c_{i}^{\sigma}$ are the points

$$
\begin{array}{lll}
b^{0 i} & \ldots & b^{i i} \\
b^{0 i+1} & \ldots & b^{i i+1} \\
\ldots & \ldots & \ldots \\
b^{0 n} & \ldots & b^{i n}
\end{array}
$$


The faces of $c_{i}^{\sigma}$ are the convex hulls of the sets of vertices lying at the crossings of a collection of rows with a collection of columns of (4.2). The $n$-cell $c_{i}^{\sigma}$ is contained in $\sigma$.

Proof. Since $\chi_{i}^{\sigma} \mid\left[v_{0}, \ldots, v_{i}\right]$ is an affine isomorphism, $0 \leq i \leq n$, and $\chi_{i}^{\sigma}\left(v_{j}\right)=\frac{1}{2}\left(v_{i}+v_{j}\right)=b^{j i}, 0 \leq j \leq i$, we see that ${c^{\prime}}_{i}^{\sigma}=\chi_{i}^{\sigma}\left[v_{0}, \ldots, v_{i}\right]=$ $\left[b^{0 i}, \ldots, b^{i i}\right]$ is a simplex with vertices $b^{0 i}, \ldots, b^{i i}$. Analogously, $c_{i}^{\prime \prime \sigma}=$ $\chi_{i}^{\sigma}\left[v_{i}, \ldots, v_{n}\right]=\left[b^{i i}, \ldots, b^{i n}\right]$ is a simplex with vertices $b^{i i}, \ldots, b^{i n}$. Therefore, $c_{i}^{\sigma}={c^{\prime}}_{i} \oplus \oplus{c^{\prime \prime}}_{i}$ is a convex polytope of dimension $n$ with vertices $b^{j i}+b^{i k}-v_{i}=\frac{1}{2}\left(v_{j}+v_{k}\right)=b^{j k}$, where $0 \leq j \leq i$ and $i \leq k \leq n$. Since $b^{j k} \in \sigma$, it follows that $c_{i}^{\sigma} \subseteq \sigma$.

We will refer to (4.2) as to the vertex scheme of $c_{i}^{\sigma}$. Similarly, we will refer to the crossings of the rows and columns of (4.2), which span a face of $c_{i}^{\sigma}$ as to the vertex scheme of that face. Note that when $\sigma=\Delta^{n}$, then $P_{i}^{n}, Q_{i}^{i}$ and $R_{i}^{n-i}$, defined in Section 4 of [7], coincide with $c_{i}^{\sigma}, c_{i}^{\prime \sigma}$ and $c_{i}^{\prime \prime \sigma}$.

If $\sigma$ is the standard $n$-simplex $\Delta^{n}=\left[e_{0}, \ldots, e_{n}\right]$, then $c_{i}^{\sigma}$ is just the convex polytope $P_{i}^{n}$, defined in [5], I.1.3, using barycentric coordinates $\lambda_{0}, \ldots, \lambda_{n}$ (with respect to $\Delta^{n}$ ) of points $u \in \sigma$ and formula (4.3). This assertion follows from the next lemma, putting $\sigma=\left[e_{0}, \ldots, e_{n}\right]$.

Lemma 4.2. A point $u \in \sigma$ belongs to $c_{i}^{\sigma}, 0 \leq i \leq n$, if and only if its barycentric coordinates $\lambda_{0}, \ldots, \lambda_{n}$ with respect to $\sigma$ satisfy the following condition,

$$
\sum_{j=0}^{j=i-1} \lambda_{j} \leq \frac{1}{2} \leq \sum_{j=0}^{j=i} \lambda_{j} .
$$

Proof. Assume that (4.3) holds and consider the points

$$
\begin{aligned}
w^{\prime} & =\sum_{j=0}^{j=i-1} 2 \lambda_{j} v_{j}+\left(1-\sum_{j=0}^{j=i-1} 2 \lambda_{j}\right) v_{i} \in \operatorname{Aff}\left[v_{0}, \ldots, v_{i}\right], \\
w^{\prime \prime} & =\sum_{j=i+1}^{j=n} 2 \lambda_{j} v_{j}+\left(1-\sum_{j=i+1}^{j=n} 2 \lambda_{j}\right) v_{i} \in \operatorname{Aff}\left[v_{i}, \ldots, v_{n}\right] .
\end{aligned}
$$

Since $\sum_{j=0}^{j=n} \lambda_{j}=1$, (4.3) implies $1-\sum_{j=0}^{j=i-1} 2 \lambda_{j} \geq 0$ and $1-\sum_{j=i+1}^{j=n} 2 \lambda_{j} \geq 0$, which shows that $w^{\prime} \in\left[v_{0}, \ldots, v_{i}\right]$ and $w^{\prime \prime} \in\left[v_{i}, \ldots, v_{n}\right]$. Defining points $u^{\prime}, u^{\prime \prime} \in \sigma$, by putting $u^{\prime}-v_{i}=\frac{1}{2}\left(w^{\prime}-v_{i}\right), u^{\prime \prime}-v_{i}=\frac{1}{2}\left(w^{\prime \prime}-v_{i}\right)$, we conclude that $u^{\prime} \in c_{i}^{\prime \sigma}$ and $u^{\prime \prime} \in c_{i}^{\prime \prime \sigma}$. Moreover, (4.4) and (4.5) imply that $u^{\prime}+u^{\prime \prime}-v_{i}=$ $\frac{1}{2}\left(w^{\prime}+w^{\prime \prime}\right)=\sum_{j=0}^{j=n} \lambda_{j} v_{j}=u$, which shows that $u \in c_{i}^{\prime \sigma} \oplus c_{i}^{\prime \prime}=c_{i}^{\sigma}$.

Conversely, if $u \in c_{i}^{\sigma}$, then $u$ is of the form $u=u^{\prime}+u^{\prime \prime}-v_{i}$, where $u^{\prime} \in c_{i}^{\prime \sigma}, u^{\prime \prime} \in c_{i}^{\prime \prime}$. Therefore, there exist points $w^{\prime} \in\left[v_{0}, \ldots, v_{i}\right]$ and $w^{\prime \prime} \in$ $\left[v_{i}, \ldots, v_{n}\right]$ such that $u^{\prime}-v_{i}=\frac{1}{2}\left(w^{\prime}-v_{i}\right), u^{\prime \prime}-v_{i}=\frac{1}{2}\left(w^{\prime \prime}-v_{i}\right)$. Let $\mu_{0}, \ldots, \mu_{i}$ and $\nu_{i}, \ldots, \nu_{n}$ be the barycentric coordinates of $w^{\prime}$ and $w^{\prime \prime}$ with respect to $\left[v_{0}, \ldots, v_{i}\right]$ and $\left[v_{i}, \ldots, v_{n}\right]$, respectively. Then $u=u^{\prime}+u^{\prime \prime}-v_{i}=\frac{1}{2}\left(w^{\prime}+\right.$ $\left.w^{\prime \prime}\right)=\frac{1}{2} \sum_{j=0}^{j=i} \mu_{j} v_{j}+\frac{1}{2} \sum_{j=i}^{j=n} \nu_{j} v_{j}$. Since $u=\sum_{j=0}^{j=n} \lambda_{j} v_{j}$, it follows that $\lambda_{j}=\frac{1}{2} \mu_{j}$, for $0 \leq j \leq i-1$, and $\lambda_{j}=\frac{1}{2} \nu_{j}$, for $i+1 \leq j \leq n$. Consequently, 
$\sum_{j=0}^{j=i-1} \lambda_{j}=\frac{1}{2} \sum_{j=0}^{j=i-1} \mu_{j} \leq \frac{1}{2}$ and $\sum_{j=i+1}^{j=n} \lambda_{j}=\frac{1}{2} \sum_{j=i+1}^{j=n} \nu_{j} \leq \frac{1}{2}$ and thus, $\sum_{j=0}^{j=i} \lambda_{j}=1-\sum_{j=i+1}^{j=n} \lambda_{j} \geq 1-\frac{1}{2}=\frac{1}{2}$.

4.2. The main result in this section is the following theorem.

THEOREM 4.3. For any ordered n-simplex $\sigma \in K$, the $n$-cells $c_{i}^{\sigma}, 0 \leq i \leq$ $n$, together with their faces, form a cellular complex $N(\sigma)$, which has $\sigma$ for its carrier, i.e., $|N(\sigma)|=\sigma$. If $\zeta<\sigma$ is a proper face of $\sigma$, whose ordering is induced by the ordering of $\sigma$, then $N(\zeta) \subseteq N(\sigma)$ and

$$
N(\zeta)=\zeta \cap N(\sigma)=\{\zeta \cap c \mid c \in N(\sigma)\} .
$$

If $K$ is an ordered simplicial complex in $V$, then the union $N=\cup_{\sigma \in K} N(\sigma)$ is a cellular subdivision of $K$.

Proof of THEOREM 4.3

4.2.1. Let us first prove that the intersection $c_{i}^{\sigma} \cap c_{i^{\prime}}^{\sigma}$ is a common face of both $n$-cells $c_{i}^{\sigma}$ and $c_{i^{\prime}}^{\sigma}$. It suffices to consider the case when $i<i^{\prime}$. We will define an $(n-1)$-dimensional affine manifold $S \subseteq$ Aff $(\sigma)$ such that $S$ is a supporting hyperplane for both convex sets $c_{i}^{\sigma}$ and $c_{i^{\prime}}^{\sigma}$ and thus, $c_{i}^{\sigma} \cap S$ is a face of $c_{i}^{\sigma}$ and $c_{i^{\prime}}^{\sigma} \cap S$ is a face of $c_{i^{\prime}}^{\sigma}$. Moreover, we will show that the sets $c_{i}^{\sigma}$ and $c_{i^{\prime}}^{\sigma}$ lie in different halfspaces $S^{+}, S^{-}$of Aff $(\sigma)$ determined by $S$. Since $S^{+} \cap S^{-}=S$, it follows that $c_{i}^{\sigma} \cap c_{i^{\prime}}^{\sigma}=\left(c_{i}^{\sigma} \cap S\right) \cap\left(c_{i^{\prime}}^{\sigma} \cap S\right)$. We will also show that the intersection $\left(c_{i}^{\sigma} \cap S\right) \cap\left(c_{i^{\prime}}^{\sigma} \cap S\right)$ is a common face of $c_{i}^{\sigma} \cap S \leq c_{i}^{\sigma}$ and $c_{i^{\prime}}^{\sigma} \cap S \leq c_{i^{\prime}}^{\sigma}$ and thus, $c_{i}^{\sigma} \cap c_{i^{\prime}}^{\sigma}$ is a common face of $c_{i}^{\sigma}$ and $c_{i^{\prime}}^{\sigma}$.

To define $S, S^{+}$and $S^{-}$, consider the function $\Lambda: \operatorname{Aff}(\sigma) \rightarrow \mathbb{R}$, where $\Lambda(u)=\sum_{0 \leq j \leq i} \lambda_{j}$. Put $S=\left\{u \in \operatorname{Aff}(\sigma) \mid \Lambda(u)=\frac{1}{2}\right\}, S^{+}=\{u \in$ $\left.\operatorname{Aff}(\sigma) \mid \Lambda(u) \geq \frac{1}{2}\right\}$ and $S^{-}=\left\{u \in \operatorname{Aff}(\sigma) \mid \Lambda(u) \leq \frac{1}{2}\right\}$. For the vertices $b^{j k}$ of $c_{i}^{\sigma}, 0 \leq j \leq i, i \leq k \leq n, \Lambda\left(b^{j k}\right)=1$, if $k=i$ and $\Lambda\left(b^{j k}\right)=\frac{1}{2}$, if $i<k$. Consequently, $\Lambda(u) \geq \frac{1}{2}$, for every point $u \in c_{i}^{\sigma}$ and thus, $c_{i}^{\sigma} \subseteq S^{+}$. Similarly, for the vertices $b^{j k}$ of $c_{i^{\prime}}^{\sigma}, 0 \leq j \leq i^{\prime}, i^{\prime} \leq k \leq n, \Lambda\left(b^{j k}\right)=\frac{1}{2}$, if $j \leq i$, and $\Lambda\left(b^{j k}\right)=0$, if $i<j$. Consequently, $\Lambda(u) \leq \frac{1}{2}$, for every point $u \in c_{i^{\prime}}^{\sigma}$ and thus, $c_{i^{\prime}}^{\sigma} \subseteq S^{-}$. Note that $b^{0 n}$ belongs to both sets $c_{i}^{\sigma} \cap S$ and $c_{i^{\prime}}^{\sigma} \cap S$, which shows that these sets are not empty. It follows that $S$ is a supporting hyperplane for both $n$-cells $c_{i}^{\sigma}$ and $c_{i^{\prime}}^{\sigma}$.

Let us denote by $W_{i}$ the set of all vertices $b^{j k} \in c_{i}^{\sigma}$ which belong to $S$ and by $W_{i^{\prime}}$ the set of all vertices $b^{j k} \in c_{i^{\prime}}^{\sigma}$, which belong to $S$. Then $c_{i}^{\sigma} \cap S=\operatorname{Conv}\left(W_{i}\right)$ and $c_{i^{\prime}}^{\sigma} \cap S=\operatorname{Conv}\left(W_{i^{\prime}}\right)$ (see [9, Lemma 6.2.3]). The above stated results show that $b^{j k} \in W_{i}$, if and only if $0 \leq j \leq i$ and $i+1 \leq k \leq n$, while $b^{j k} \in W_{i^{\prime}}$, if and only if $0 \leq j \leq i$ and $i^{\prime} \leq k \leq n$. Since $i+1 \leq i^{\prime}$, we see that $W_{i^{\prime}} \subseteq W_{i}$ and thus, $W_{i} \cap W_{i^{\prime}}=W_{i^{\prime}}$ consists of the last $n-i^{\prime}+1$ rows of the vertex scheme of $c_{i}^{\sigma}$, which coincides with the first $i+1$ columns of the vertex scheme of $c_{i^{\prime}}^{\sigma}$. Consequently, $c_{i^{\prime}}^{\sigma} \cap S=\operatorname{Conv}\left(W_{i^{\prime}}\right)$ is a face of $c_{i}^{\sigma} \cap S=\operatorname{Conv}\left(W_{i}\right)$ and thus, $c_{i^{\prime}}^{\sigma} \cap c_{i}^{\sigma}=\left(c_{i^{\prime}}^{\sigma} \cap S\right) \cap\left(c_{i}^{\sigma} \cap S\right)=c_{i^{\prime}}^{\sigma} \cap S \leq c_{i}^{\sigma} \cap S$. 
It follows that $c_{i^{\prime}}^{\sigma} \cap c_{i}^{\sigma}$ is a common face of $c_{i^{\prime}}^{\sigma}$ and $c_{i}^{\sigma}$. This completes the proof that $N(\sigma)$ is a cellular complex.

Let us now show that the union of the $n$-cells $c_{i}^{\sigma}, 0 \leq i \leq n$, equals $\sigma$ and thus, $|N(\sigma)|=\sigma$. Let $u \in \sigma$ and let $\lambda_{0}, \ldots, \lambda_{n}$ be its barycentric coordinates with respect to $\sigma$. Since $\sum_{0 \leq j \leq n} \lambda_{j}=1>\frac{1}{2}$, there is a smallest index $i$, $0 \leq i \leq n$, such that $\sum_{0 \leq j \leq i} \lambda_{j}>\frac{1}{2}$. Therefore, $\sum_{0 \leq j \leq i-1} \lambda_{j} \leq \frac{1}{2}$. Hence, Lemma 4.2 shows that $u \in \bar{c}_{i}^{\sigma}$.

4.2.2. The next lemma will complete the proof of Theorem 4.3.

LEMmA 4.4. If $\sigma \in K$ and $\zeta<\sigma$ is a proper face of $\sigma$, whose ordering is induced by the ordering of $\sigma$, then $N(\zeta) \subseteq N(\sigma)$ and formula (4.6) holds. For any cell $c \in N(\sigma)$, the intersection $\zeta \cap c$ is a face of $c$, which belongs to $N(\zeta)$. If $K$ is an ordered simplicial complex in $V$, then the union $N=\cup_{\sigma \in K} N(\sigma)$ is a cellular subdivision of $K$.

Proof. Consider an ordered simplex $\sigma$ in $V$ and a proper face $\zeta<\sigma$, whose ordering is induced by the ordering of $\sigma$. In order to prove that $N(\zeta) \subseteq$ $N(\sigma)$, it suffices to prove this assertion in the special case when $\operatorname{dim} \sigma-\operatorname{dim} \zeta=$ 1. Indeed, if $\operatorname{dim} \sigma-\operatorname{dim} \zeta=k>1$, we consider a sequence of faces $\zeta=\zeta_{k}<$ $\ldots<\zeta_{1}<\sigma$, where the dimensions of consecutive members differ by 1 . By the special case, one concludes that $N(\zeta)=N\left(\zeta_{k}\right) \subseteq \ldots \subseteq N\left(\zeta_{1}\right) \subseteq N(\sigma)$. Now assume that $\sigma=\left[v_{0}, \ldots, v_{n}\right]$ and $\zeta$ is the $(n-1)$-dimensional face of $\sigma$, which does not contain the vertex $v_{l}$, where $0 \leq l \leq n$, i.e., $\zeta=S \cap \sigma$, where $S$ is the $(n-1)$-dimensional affine manifold $S=\left\{u \in \operatorname{Aff}(\sigma) \mid \lambda_{l}(u)=0\right\}$. Since every cell $c_{1}$ from $N(\zeta)$ is a face of an $(n-1)$-cell $c$ from $N(\zeta)$, it suffices to show that every $(n-1)$-cell $c \in N(\zeta)$ belongs to $N(\sigma)$, because then $c_{1} \leq c$ will imply $c_{1} \in N(\sigma)$.

Let $c=c_{v_{i}}^{\zeta}$, where $0 \leq i \leq n$ and $i \neq l$. By Lemma 4.1, the vertex scheme of $c=c_{v_{i}}^{\zeta}$ is obtained from the vertex scheme (4.2) of $c_{v_{i}}^{\sigma}$ by deleting the row $b^{0 l} \ldots b^{i l}$, when $i<l$ and by deleting the column $b^{l i} \ldots b^{l n}$, when $i>l$. In both cases we see that $c_{v_{i}}^{\zeta} \leq c_{v_{i}}^{\sigma}$. Since $c_{v_{i}}^{\sigma} \in N(\sigma)$, we conclude that also

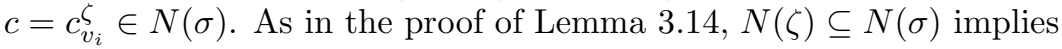

$$
N(\zeta) \subseteq \zeta \cap N(\sigma)
$$

We will now show that, for any cell $c \in N(\sigma)$, the intersection $\zeta \cap c$ is a face of $c$, which belongs to $N(\zeta)$. By the argument given in the proof of Lemma 3.14, it suffices to prove the assertion in the special case when $\operatorname{dim} \sigma-\operatorname{dim} \zeta=1$. Moreover, one can assume that $c$ is an $n$-cell from $N(\sigma)$. Indeed, an arbitrary cell $c$ from $N(\sigma)$ is a face of an $n$-cell $c^{\prime} \in N(\sigma)$. By the special case, $\zeta \cap c^{\prime}$ is a face of $c^{\prime}$, which belongs to $N(\zeta)$. Note that $c=c^{\prime} \cap c$ and thus, $\zeta \cap c=\left(\zeta \cap c^{\prime}\right) \cap c$. Since $\zeta \cap c^{\prime}$ and $c$ are faces of $c^{\prime} \in N(\sigma)$, it follows that $\zeta \cap c^{\prime}, c \in N(\sigma)$ and their intersection $\left(\zeta \cap c^{\prime}\right) \cap c$ is a face of $\zeta \cap c^{\prime}$ and $c$. Since $\zeta \cap c^{\prime} \in N(\zeta)$, it follows that also $\zeta \cap c=\left(\zeta \cap c^{\prime}\right) \cap c \in N(\zeta)$. 
Let us now assume that $\sigma=\left[v_{0}, \ldots, v_{n}\right], n \geq 1, \zeta$ is the $(n-1)$ dimensional face of $\sigma$, which does not contain the vertex $v_{l}, 0 \leq l \leq n$, and $c=c_{v_{i}}^{\sigma}, 0 \leq i \leq n$, is an $n$-cell of $N(\sigma)$. Note that $\zeta=S \cap \sigma$, where $S$ is the $(n-1)$-dimensional affine manifold $S=\left\{u \in \operatorname{Aff}(\sigma) \mid \lambda_{l}(u)=0\right\}$. Since $c \subseteq \sigma$ implies $c=\sigma \cap c$, it follows that $\zeta \cap c=S \cap c$.

We will first assume that $i=l$. In the special cases, when $0=i=l<n$ or $0<i=l=n$, the intersection $\zeta \cap c=\emptyset$ and our assertion holds. Indeed, if $0=i=l<n$, the vertex scheme of $c$ consists of a single column $b^{00} \ldots b^{0 n}$. Since $\lambda_{l}\left(b^{0 j}\right)=\lambda_{0}\left(b^{0 j}\right)>0$, for $0 \leq j \leq n$, we see that all vertices of $c$ are contained in the convex set $S^{>}=\left\{u \in \operatorname{Aff}(\sigma) \mid \lambda_{l}(u)>0\right\}$ and thus, $c \subseteq S^{>}$. However, $S^{>}$does not intersect $\zeta \subseteq S=\left\{u \in \operatorname{Aff}(\sigma) \mid \lambda_{l}(u)=0\right\}$ and thus, $c \cap \zeta=\emptyset$. Similarly, if $0<i=l=n$, the vertex scheme of $c$ consists of a single row $b^{0 n} \ldots b^{n n}$. Now $\lambda_{l}\left(b^{j n}\right)=\lambda_{n}\left(b^{j n}\right)>0$, for $0 \leq j \leq n$. Consequently, $c \subseteq S^{>}$and again $c \cap \zeta=\emptyset$.

Now assume that $0<i=l<n$. Then $b^{0 n}=\frac{1}{2}\left(v_{0}+v_{n}\right) \in \zeta$, because $v_{0}, v_{n} \in \zeta$. Since $b^{0 n}$ is a vertex of $c$, it follows that $\zeta \cap c \neq \emptyset$. Clearly, $c$ and $\sigma$ are contained in the affine halfspace $S^{+}=\left\{u \in \operatorname{Aff}(\sigma) \mid \lambda_{l}(u) \geq 0\right\}$ and thus, $S$ is a supporting hyperplane, for the $n$-cell $c=c_{v_{i}}^{\sigma}$. This implies that $\zeta \cap c=c \cap S$ is the face of $c$, whose vertices are all the vertices $b^{j k}$ of $c$, which are contained in $S$, i.e., for which $\lambda_{l}\left(b^{j k}\right)=0$. These vertices are obtained from the vertex scheme (4.2) by deleting the first row and the last column, i.e., $\zeta \cap c$ is the face of $c$, having the following vertex scheme.

$$
\begin{array}{lll}
b^{0 l+1} & \ldots & b^{l-1 l+1} \\
\ldots & \ldots & \ldots \\
b^{0 n} & \ldots & b^{l-1 n} .
\end{array}
$$

Putting on top of (4.8) the row $b^{0 l-1} \ldots b^{l-1 l-1}$, we obtain the vertex scheme of $c_{v_{l-1}}^{\zeta} \in N(\zeta)$. Consequently, $\zeta \cap c$ is a face of $c_{v_{l-1}}^{\zeta}$ and thus, it also belongs to $N(\zeta)$.

It remains to consider the case when $i \neq l$. In that case $b^{i i}=v_{i}$ is a vertex of $\zeta$ and thus, $S \cap c=\zeta \cap c \neq \emptyset$. Since $c, \sigma \subset S^{+}$, one concludes that $S$ is a supporting hyperplane, for the $n$-cell $c=c_{v_{i}}^{\sigma}$. Consequently, $\zeta \cap c=c \cap S$ is the face of $c$, whose vertices are all the vertices $b^{j k}$ of $c$, which are contained in $S$, i.e., for which $\lambda_{l}\left(b^{j k}\right)=0$. If $i<l$, these are all the vertices from (4.2), except the ones belonging to the row $b^{0 l} \ldots b^{i l}$. However, these are just the vertices of $c_{v_{i}}^{\zeta} \in N(\zeta)$ and thus, $\zeta \cap c=c_{v_{i}}^{\zeta}$. The same formula holds if $l<i$, because in that case, the vertices $b^{j k}$ of $c$, for which $\lambda_{l}\left(b^{j k}\right)=0$, are all the vertices in (4.2), except the ones belonging to the column $b^{l i} \ldots b^{l n}$. However, these are just the vertices of $c_{v_{i}}^{\zeta} \in N(\zeta)$. Hence, for $i \neq l, \zeta \cap c=c_{v_{i}}^{\zeta}$ belongs to $N(\zeta)$.

It is an immediate consequence of the assertion we just proved that

$$
\zeta \cap N(\sigma) \subseteq N(\zeta)
$$


Clearly, (4.6) follows from (4.7) and (4.9). The final assertion of Lemma 4.4 that $N=\cup_{\sigma \in K} N(\sigma)$ is a cellular subdivision of $K$ is an immediate consequence of (4.6).

\section{The FiRst ITERATED CANONICAL SUBDIVISION $L^{\prime}(K)$}

5.1. In this section we first extend the definition of the cellular complex $L(\sigma)$, associated with a simplex $\sigma \subseteq V$ (considered in Section 3), to the case of a cellular complex $L\left(\sigma \oplus \sigma^{\prime}\right)$, associated with the direct sum $\sigma \oplus \sigma^{\prime}$ of an ordered pair $\left(\sigma, \sigma^{\prime}\right)$ of simplices from $V$ of $\operatorname{dimensions} \operatorname{dim} \sigma=k$ and $\operatorname{dim} \sigma^{\prime}=k^{\prime}$. We assume that $\sigma \oplus \sigma^{\prime}$ is well defined, i.e., $\operatorname{Aff}(\sigma) \cap \operatorname{Aff}\left(\sigma^{\prime}\right)$ is a single point $w$ and thus, $\sigma \oplus \sigma^{\prime}=\sigma+\sigma^{\prime}-w$. We will first define the $n$-cells of $L\left(\sigma \oplus \sigma^{\prime}\right)$, where $n=k+k^{\prime}$. Then $L\left(\sigma \oplus \sigma^{\prime}\right)$ will consist of all faces of these $n$-cells. By definition, an $n$-cell of $L\left(\sigma \oplus \sigma^{\prime}\right)$ is a convex polytope of the form $c \oplus \sigma^{\prime}$, where $c$ is a $k$-cell from $L(\sigma)$. Clearly, $\operatorname{Aff}(c)=\operatorname{Aff}(\sigma)$ and thus, $\operatorname{Aff}(c) \cap \operatorname{Aff}\left(\sigma^{\prime}\right)=\{w\}$. Consequently, $c \oplus \sigma^{\prime}=c+\sigma^{\prime}-w$ is a well-defined $n$-dimensional convex polytope. If we denote by $L_{n}\left(\sigma \oplus \sigma^{\prime}\right)$ the set of all $n$-cells of $L\left(\sigma \oplus \sigma^{\prime}\right)$ and we denote by $L_{k}(\sigma)$ the set of all $k$-cells of $L(\sigma)$, we put

$$
L_{n}\left(\sigma \oplus \sigma^{\prime}\right)=L_{k}(\sigma) \oplus \sigma^{\prime}=\left\{c \oplus \sigma^{\prime} \mid c \in L_{k}(\sigma)\right\} .
$$

In other words, $L_{n}\left(\sigma \oplus \sigma^{\prime}\right)$ is obtained from $\sigma \oplus \sigma^{\prime}$ by applying the operation $L_{k}$ to the first summand $\sigma$, leaving the second summand $\sigma^{\prime}$ unchanged. If $\sigma^{\prime}$ is a 0 -dimensional simplex $*^{\prime}$, then $\operatorname{Aff}\left(\sigma^{\prime}\right)=*^{\prime}$. The reference point of $\sigma \oplus \sigma^{\prime}$ is $*^{\prime}$, because $\operatorname{Aff}(\sigma) \cap \operatorname{Aff}\left(*^{\prime}\right)=*^{\prime}$. Consequently, for $c \in L_{n}(\sigma)$, the direct sum $c \oplus *^{\prime}=c+*^{\prime}-*^{\prime}=c$ and thus, $L_{n}\left(\sigma \oplus *^{\prime}\right)=\left\{c \oplus *^{\prime} \mid c \in L_{n}(\sigma)\right\}=$ $\left\{c \mid c \in L_{n}(\sigma)\right\}=L_{n}(\sigma)$. Therefore, $L\left(\sigma \oplus *^{\prime}\right)=L(\sigma)$ and we see that $L(\sigma)$ is a special case of $L\left(\sigma \oplus \sigma^{\prime}\right)$.

To show that $L\left(\sigma \oplus \sigma^{\prime}\right)$ is indeed a cellular complex, it suffices to show that any two $n$-cells $c \oplus \sigma^{\prime}$ and $c_{1} \oplus \sigma^{\prime}$ from $L_{n}\left(\sigma \oplus \sigma^{\prime}\right)$ intersect in a common face. Let us first show that $\left(c \oplus \sigma^{\prime}\right) \cap\left(c_{1} \oplus \sigma^{\prime}\right)=\left(c \cap c_{1}\right)+\sigma^{\prime}-w$. Indeed, $\operatorname{dim} c=\operatorname{dim} \sigma=k$ implies $\operatorname{Aff}(c)=\operatorname{Aff}(\sigma)$ and thus, $\operatorname{Aff}(c) \cap \operatorname{Aff}\left(\sigma^{\prime}\right)=$ $\operatorname{Aff}(\sigma) \cap \operatorname{Aff}\left(\sigma^{\prime}\right)=\{w\}$ so that $c \oplus \sigma^{\prime}=c+\sigma^{\prime}-w$. Analogously, $c_{1} \oplus \sigma^{\prime}=$ $c_{1}+\sigma^{\prime}-w$. Now assume that $u \in\left(c \oplus \sigma^{\prime}\right) \cap\left(c_{1} \oplus \sigma^{\prime}\right)$. Then $u=s+t-w=$ $s_{1}+t_{1}-w$, where $s \in c \subseteq \operatorname{Aff}(\sigma), s_{1} \in c_{1} \subseteq \operatorname{Aff}(\sigma)$ and $t, t_{1} \in \sigma^{\prime} \subseteq \operatorname{Aff}\left(\sigma^{\prime}\right)$. Consequently, $s-s_{1}+w=t_{1}-t+w \in \operatorname{Aff}(\sigma) \cap \operatorname{Aff}\left(\sigma^{\prime}\right)=\{w\}$ and thus, $s=s_{1} \in c \cap c_{1}, t=t_{1} \in \sigma^{\prime}$. It follows that $u=s+t-w \in\left(c \cap c_{1}\right)+\sigma^{\prime}-w$ and one obtains the inclusion $\left(c \oplus \sigma^{\prime}\right) \cap\left(c_{1} \oplus \sigma^{\prime}\right) \subseteq\left(c \cap c_{1}\right)+\sigma^{\prime}-w$. The converse inclusion is obvious. Since $c \cap c_{1}$ is a common face of $c$ and $c_{1}$, it follows that $\left(c \cap c_{1}\right)+\sigma^{\prime}-w$ is a common face of $c+\sigma^{\prime}-w=c \oplus \sigma^{\prime}$ and $c_{1}+\sigma^{\prime}-w=c_{1} \oplus \sigma^{\prime}$.

The carrier $\left|L\left(\sigma \oplus \sigma^{\prime}\right)\right|=\sigma \oplus \sigma^{\prime}$. Indeed, if $u \in \sigma \oplus \sigma^{\prime}=\sigma+\sigma^{\prime}-w$, then $u$ is of the form $u=s+t-w$, where $s \in \sigma, t \in \sigma^{\prime}$. Since $\left|L_{k}(\sigma)\right|=|L(\sigma)|=\sigma$, 
there is a cell $c \in L_{k}(\sigma)$ such that $s \in c$ and thus, $u=s+t-w \in c+\sigma^{\prime}-w=$ $c \oplus \sigma^{\prime} \in L_{n}\left(\sigma \oplus \sigma^{\prime}\right) \subseteq L\left(\sigma \oplus \sigma^{\prime}\right)$.

5.2. We will now apply the above described construction to cells $c$ from the subdivision $L(\sigma)$ of a simplex $\sigma \in K$, described in Section 3. Recall that, for an $n$-simplex $\sigma$, with every $n$-cell $c \in L_{n}(\sigma)$ is associated the ordered pair of simplices $\left(c^{\tau}, d_{\pi}^{\tau}\right)$ such that $c=c^{\tau} \oplus d_{\pi}^{\tau}, \tau \leq \sigma, \operatorname{dim} \tau=k$. Therefore, $L(c)$ is a well-defined cellular complex with carrier $|L(c)|=c$. Its $n$-cells form the set

$$
L_{n}(c)=L_{n}\left(c^{\tau} \oplus d_{\pi}^{\tau}\right)=L_{k}\left(c^{\tau}\right) \oplus d_{\pi}^{\tau} .
$$

All other cells are faces of these $n$-cells. The first iterated canonical subdivision $L^{\prime}(\sigma)$ of an $n$-simplex $\sigma \subseteq V$ is defined by the formula

$$
L^{\prime}(\sigma)=\cup_{c \in L_{n}(\sigma)} L(c) .
$$

If $K$ is a simplicial complex in $V$, the first iterated canonical subdivision $L^{\prime}(K)$ is defined by the formula

$$
L^{\prime}(K)=\cup_{\sigma \in K} L^{\prime}(\sigma) .
$$

THEOREM 5.1. If $\sigma$ is a simplex in $V$, then $L^{\prime}(\sigma)$ is a cellular subdivision of $L(\sigma)$, hence, it is also a cellular subdivision of $\sigma$. If $\zeta<\sigma$, then $L^{\prime}(\zeta) \subseteq$ $L^{\prime}(\sigma)$ and $L^{\prime}(\sigma) \cap \zeta=L^{\prime}(\zeta)$. If $K$ is a simplicial complex in $V$, then $L^{\prime}(K)$ is a cellular subdivision of $L(K)$, hence, it is also a cellular subdivision of $K$.

Proof. First note that every face $e^{*}$ of $c^{*} \in L^{\prime}(\sigma)$ also belongs to $L^{\prime}(\sigma)$. Indeed, if $\operatorname{dim} \sigma=n$, there exists an $n$-cell $c \in L_{n}(\sigma)$ such that $c^{*} \in L(c)$. Since $L(c)$ is a cellular complex, it follows that also $e^{*}$ belongs to $L(c)$ and thus, $e^{*} \in L^{\prime}(\sigma)$. To prove that $L^{\prime}(\sigma)$ is a cellular complex, we still need to prove that the intersection of two cells $c^{*}, c_{1}^{*}$ from $L^{\prime}(\sigma)$ is a common face of these cells. By definition, there exist cells $c, c_{1} \in L_{n}(\sigma) \subseteq L(\sigma)$ such that $c^{*} \in L(c)$ and $c_{1}^{*} \in L\left(c_{1}\right)$. There is no loss of generality in assuming that $c^{*}, c_{1}^{*}$ are $n$-cells, i.e., $c^{*} \in L_{n}(c), c_{1}^{*} \in L_{n}\left(c_{1}\right)$. Indeed, arbitrary cells from $L(c)$ and $L\left(c_{1}\right)$ are faces of $n$-cells from $L(c)$ and $L\left(c_{1}\right)$, respectively. If the latter cells intersect in a common face, then so do the original cells.

Since $L(\sigma)$ is a cellular complex, the intersection $a=c \cap c_{1}$ is a common face of both cells $c$ and $c_{1}$. We will define a cellular complex $L(a)$ and we will show that

$$
a \cap c^{*}, a \cap c_{1}^{*} \in L(a) .
$$

Note that (5.5) implies that the intersection $\left(a \cap c^{*}\right) \cap\left(a \cap c_{1}^{*}\right)$ is a common face of $a \cap c^{*}$ and $a \cap c_{1}^{*}$. Since $c^{*} \subseteq c$ and $c_{1}^{*} \subseteq c_{1}$, it follows that $c^{*} \cap c_{1}^{*} \subseteq c \cap c_{1}=a$ and thus, $c^{*} \cap c_{1}^{*}=\left(a \cap c^{*}\right) \cap\left(a \cap c_{1}^{*}\right)$. Consequently, (5.5) implies

$$
c^{*} \cap c_{1}^{*} \leq a \cap c^{*}, \quad c^{*} \cap c_{1}^{*} \leq a \cap c_{1}^{*} .
$$


The desired assertion that $c^{*} \cap c_{1}^{*}$ is a common face of the cells $c^{*}$ and $c_{1}^{*}$ will follow, if we also show that

$$
a \cap c^{*} \leq c^{*}, \quad a \cap c_{1}^{*} \leq c_{1}^{*} .
$$

We will now define $L(a)$. By Lemma 3.4, applied to $a \leq c=c_{\pi}^{\tau}$, there are simplices $c_{a}, d_{a}$, which are faces of $a$, such that the vertex scheme of $c_{a}$ is the first row of the vertex scheme of $a$ and $d_{a}$ is the first column of the vertex scheme of $a$. Moreover, $a=c_{a} \oplus d_{a}$ and the reference point $w$ is the only common vertex of $c_{a}$ and $d_{a}$, i.e., $w=w_{j}^{0 \ldots k \pi(k+1) \ldots \pi(k+i)}$. We define $L(a)$ by putting $L(a)=L\left(c_{a}\right) \oplus d_{a}=L\left(c_{a}\right)+d_{a}-w$.

Let us prove that the cellular complex $L(a)$ is completely determined by $a$, i.e., it does not depend on $c$. This means that, if we have another $n$-cell $c_{1}=$ $c_{\pi^{1}}^{\tau^{1}} \in L(\sigma)$ such that $a \leq c_{1}$ and perform the above construction, the resulting cellular complex $L^{1}(a)$ will coincide with $L(a)$. Indeed, this time Lemma 3.4 will give us faces $c_{a}, d_{a}^{1}$ of $a$ such that $a=c_{a} \oplus d_{a}^{1}=c_{a}+d_{a}^{1}-w^{1}$, where the reference point $w^{1}$ is the only common vertex of $c_{a}$ and $d_{a}^{1}$. Moreover, the construction will give us the cellular complex $L^{1}(a)=L\left(c_{a}\right) \oplus d_{a}^{1}=$ $L\left(c_{a}\right)+d_{a}^{1}-w^{1}$. Note that the first summand $c_{a}$ is the same as in the case of the $n$-cell $c=c_{\pi}^{\tau}$, because $c_{a}$ is completely determined by $a$ alone (see Lemma 3.4). To prove that $L^{1}(a)=L(a)$ we need to prove that $d_{a}-w=d_{a}^{1}-w^{1}$.

Since $d_{a}^{1} \leq a$, the vertex scheme of $d_{a}^{1}$ consists of the crossings of a set of rows with a set of columns of the vertex scheme (3.16) of $a$. Since $w^{1}$ is a vertex of $c_{a}$ and the vertices of $c_{a}$ fill up the first row of (3.16), it follows that $w^{1}=w_{j_{l}}^{0 \ldots k \pi(k+1) \ldots \pi(k+i)}$, for some $0 \leq l \leq r$. Moreover, we see that the set of rows includes the first row and the set of columns includes the $j_{l}$-th column. It cannot include any other column, because the crossing of that column with the first row would be a vertex of $d_{a}^{1}$, different from $w^{1}$ and belonging to $c_{a}$. However, $d_{a}^{1}$ has only one vertex in common with $c_{a}$. This proves that the vertex scheme of $d_{a}^{1}$ is a subscheme of the $j_{l}$-th column of (3.16). The number of vertices of $d_{a}^{1}$ equals $\operatorname{dim} d_{a}^{1}+1$. However, $c_{a} \oplus d_{a}=a=c_{a} \oplus d_{a}^{1}$ implies that $\operatorname{dim} d_{a}^{1}=\operatorname{dim} d_{a}=r$. Consequently, $d_{a}^{1}$ has $r+1$ vertices and thus, the vertex scheme of $d_{a}^{1}$ is the whole $j_{l}$-th column of (3.16). Finally, translation by $w_{j_{l}}^{\tau}-w_{j}^{\tau}$ is a bijection between the vertices of the $j$-th column and those of the $j_{l}$-th column, which implies that $d_{a}^{1}=d_{a}+w_{j_{l}}^{\tau}-w_{j}^{\tau}$. However, $w^{1}-w=w_{j_{l}}^{0 \ldots k \pi(k+1) \ldots \pi(k+i)}-w_{j}^{0 \ldots k \pi(k+1) \ldots \pi(k+i)}=w_{j_{l}}^{0 \ldots k}-w_{j}^{0 \ldots k}$ and we obtain the desired relation $d_{a}^{1}=d_{a}+w^{1}-w$.

We will now show that

$$
a \cap L(c)=L(a), \quad a \cap L\left(c_{1}\right)=L(a) .
$$

Recall that $c=c^{\tau} \oplus d_{\pi}^{\tau}=c^{\tau}+d_{\pi}^{\tau}-w_{0}^{\tau}$ and thus, $L(c)=L\left(c^{\tau}\right) \oplus d_{\pi}^{\tau}=$ $L\left(c^{\tau}\right)+d_{\pi}^{\tau}-w_{0}^{\tau}$. Let $c^{\prime}{ }_{a}, d^{\prime}{ }_{a}, c_{a}$ and $d_{a}$ be as in Lemma 3.4. Then $c^{\prime}{ }_{a} \leq c^{\tau}$, 
$d^{\prime}{ }_{a} \leq d_{\pi}^{\tau}, a={c^{\prime}}^{\prime}{ }_{a}+d^{\prime}{ }_{a}-w_{0}^{\tau}$ and thus, by 2.7 ,

$$
\begin{aligned}
a \cap L(c) & =\left(c^{\prime}{ }_{a}+d^{\prime}{ }_{a}-w_{0}^{\tau}\right) \cap\left(L\left(c^{\tau}\right)+d_{\pi}^{\tau}-w_{0}^{\tau}\right) \\
& =\left(c^{\prime}{ }_{a} \cap L\left(c^{\tau}\right)\right)+\left(d^{\prime}{ }_{a} \cap d_{\pi}^{\tau}\right)-w_{0}^{\tau} .
\end{aligned}
$$

By (3.17) (with $\sigma=c^{\tau}$ and $\left.\zeta=c^{\prime}{ }_{a}\right), c^{\prime}{ }_{a} \cap L\left(c^{\tau}\right)=L\left(c^{\prime}{ }_{a}\right)$. Moreover, $d^{\prime}{ }_{a} \cap d_{\pi}^{\tau}=$ $d^{\prime}{ }_{a}$ and we see that $a \cap L(c)=L\left(c^{\prime}{ }_{a}\right)+d^{\prime}{ }_{a}-w_{0}^{\tau}$. On the other hand, since $a=c_{a} \oplus d_{a}$ and $w_{j}^{0 \ldots k \pi(k+1) \ldots \pi(k+i)}$ is the corresponding reference point, it follows that $L(a)=L\left(c_{a}\right) \oplus d_{a}=L\left(c_{a}\right)+d_{a}-w_{j}^{0 \ldots k \pi(k+1) \ldots \pi(k+i)}$. Taking into account that $d_{a}=d^{\prime}{ }_{a}+w_{j}^{\tau}-w_{0}^{\tau}$, we see that $L(a)=L\left(c_{a}\right)+\left(d^{\prime}{ }_{a}+w_{j}^{\tau}-\right.$ $\left.w_{0}^{\tau}\right)-w_{j}^{0 \ldots k \pi(k+1) \ldots \pi(k+i)}=L\left(c_{a}\right)+d^{\prime}{ }_{a}-w_{0}^{0 \ldots k \pi(k+1) \ldots \pi(k+i)}$. Now note that translation by $w_{0}^{0 \ldots k \pi(k+1) \ldots \pi(k+i)}-w_{0}^{0 \ldots k}$ is a bijection between the vertices of $c^{\prime}{ }_{a}$ and $c_{a}$ (see Lemma 3.4) and thus, it is an affine isomorphism between the simplices $c^{\prime}{ }_{a}$ and $c_{a}$. Therefore, $L\left(c_{a}\right)=L\left(c^{\prime}{ }_{a}\right)+w_{0}^{0 \ldots k \pi(k+1) \ldots \pi(k+i)}-$ $w_{0}^{0 \ldots k}$. It follows that $L(a)=\left(L\left(c^{\prime}{ }_{a}\right)+w_{0}^{0 \ldots k \pi(k+1) \ldots \pi(k+i)}-w_{0}^{0 \ldots k}\right)+d^{\prime}{ }_{a}-$ $w_{0}^{0 \ldots k \pi(k+1) \ldots \pi(k+i)}=L\left(c^{\prime}{ }_{a}\right)+d^{\prime}{ }_{a}-w_{0}^{0 \ldots k}=a \cap L(c)$. This establishes the first of the two relations in (5.8). The second one, follows by symmetry.

Since $c^{*} \in L(c)$, we see that $a \cap c^{*} \in a \cap L(c)=L(a)$. Analogously, $a \cap c_{1}^{*} \in a \cap L\left(c_{1}^{*}\right)=L(a)$. Consequently, $a \cap c^{*}$ and $a \cap c_{1}^{*}$ are cells of the cellular complex $L(a)$, i.e., (5.5) holds. To complete the proof that $c^{*} \cap c_{1}^{*}$ is a common face of $c^{*}$ and $c_{1}^{*}$, it remains to prove (5.7). Since $L(c)=L\left(c^{\tau}\right)+d_{\pi}^{\tau}-w_{0}^{\tau}$, every $n$-cell $c^{*} \in L(c)$ is of the form $c^{*}=c^{\prime}+d_{\pi}^{\tau}-w_{0}^{\tau}$, where $c^{\prime} \in L\left(c^{\tau}\right)$. Taking into account that $a=c^{\prime}{ }_{a}+d^{\prime}{ }_{a}-w_{0}^{\tau}$, where $c^{\prime}{ }_{a} \leq c^{\tau}$ and $d^{\prime}{ }_{a} \leq d_{\pi}^{\tau}$, we see that $a \cap c^{*}=\left(c^{\prime}{ }_{a}+d^{\prime}{ }_{a}-w_{0}^{\tau}\right) \cap\left(c^{\prime}+d_{\pi}^{\tau}-w_{0}^{\tau}\right)=e+d^{\prime}{ }_{a}-w_{0}^{\tau}$, where $e=c^{\prime}{ }_{a} \cap c^{\prime} \in c^{\prime}{ }_{a} \cap L\left(c^{\tau}\right)$. Since $c^{\tau}$ is a simplex, $c^{\prime} \in L\left(c^{\tau}\right)$ and $c^{\prime}{ }_{a} \leq c^{\tau}$, Lemma 3.14 (with $c^{\tau}, c^{\prime}{ }_{a}$ and $c^{\prime}$ as $\sigma, \zeta$ and $c$ ) shows that $c^{\prime}{ }_{a} \cap L\left(c^{\tau}\right)=L\left(c^{\prime}{ }_{a}\right)$. Since $c^{\prime}{ }_{a} \leq c^{\tau}$, we see that $L\left(c^{\prime}{ }_{a}\right) \subseteq L\left(c^{\tau}\right)$ and thus, $e=c^{\prime}{ }_{a} \cap c^{\prime}$ is a cell of the cellular complex $L\left(c^{\tau}\right)$. However, $c^{\prime}$ is also a cell of $L\left(c^{\tau}\right)$. Therefore, the intersection $e \cap c^{\prime}=e$ of these two cells is a common face of both cells. In particular, $e=c^{\prime} \cap c^{\prime}{ }_{a} \leq c^{\prime}$. Since $d^{\prime}{ }_{a} \leq d_{\pi}^{\tau}$, we conclude that $a \cap c^{*}=$ $e+d^{\prime}{ }_{a}-w_{0}^{\tau} \leq c^{\prime}+d_{\pi}^{\tau}-w_{0}^{\tau}=c^{*}$. By symmetry, we also have $a \cap c_{1}^{*} \leq c_{1}^{*}$.

5.3. $L^{\prime}(\sigma)$ is a subdivision of $L(\sigma)$, because the carrier $|L(c)|=c$, for $c \in L_{n}(\sigma)$. Since $L(\sigma)$ is a subdivision of $\sigma$, it follows that $L^{\prime}(\sigma)$ is also a subdivision of $\sigma$. That $\zeta<\sigma$ implies $L^{\prime}(\zeta) \subseteq L^{\prime}(\sigma)$ is an immediate consequence of definition (5.3) (for $\zeta$ and $\sigma$ ) and of the fact that $\zeta<\sigma$ implies $L(\zeta) \subseteq L(\sigma)$ (see Theorem 3.6). Since all cells in $L^{\prime}(\zeta)$ are contained in $\zeta$, we see that $L^{\prime}(\zeta) \cap \zeta=L^{\prime}(\zeta)$ and thus, $L^{\prime}(\zeta) \subseteq L^{\prime}(\sigma)$ implies

$$
L^{\prime}(\zeta) \subseteq L^{\prime}(\sigma) \cap \zeta .
$$

To prove the opposite inclusion

$$
L^{\prime}(\sigma) \cap \zeta \subseteq L^{\prime}(\zeta)
$$


and thus, obtain the equality $L^{\prime}(\sigma) \cap \zeta=L^{\prime}(\zeta)$, it suffices to prove that $e^{*} \in L^{\prime}(\sigma)$ implies $e^{*} \cap \zeta \in L^{\prime}(\zeta)$ in the special case when $\operatorname{dim} e^{*}=n$. Indeed, every cell $e^{*} \in L^{\prime}(\sigma)$ is the face of an $n$-cell $c^{*} \in L^{\prime}(\sigma)$. Since $e^{*} \subseteq c^{*}$, it follows that $e^{*}=e^{*} \cap c^{*}$ and thus, $e^{*} \cap \zeta=e^{*} \cap\left(c^{*} \cap \zeta\right)$. By the special case, $c^{*} \cap \zeta \in L^{\prime}(\zeta) \subseteq L^{\prime}(\sigma)$. Since $L^{\prime}(\sigma)$ is a cellular complex and $e^{*}, c^{*} \cap \zeta$ are two cells of $L^{\prime}(\sigma)$, their intersection $e^{*} \cap\left(c^{*} \cap \zeta\right)=e^{*} \cap \zeta$ is a common face of $e^{*}$ and $c^{*} \cap \zeta$. In particular, $e^{*} \cap \zeta$ is a face of $c^{*} \cap \zeta$. Since $c^{*} \cap \zeta \in L^{\prime}(\zeta)$ and $L^{\prime}(\zeta)$ is a cellular complex, it follows that also $e^{*} \cap \zeta \in L^{\prime}(\zeta)$.

Now assume that $c^{*} \in L^{\prime}(\sigma), \operatorname{dim} c^{*}=n$. To prove that $c^{*} \cap \zeta \in L^{\prime}(\zeta)$, it suffices to prove the assertion in the special case when $\operatorname{dim} \sigma-\operatorname{dim} \zeta=1$. Indeed, if $\operatorname{dim} \sigma-\operatorname{dim} \zeta=k>1$, then we consider a sequence of faces $\zeta=$ $\zeta_{k}<\ldots<\zeta_{1}<\sigma$, where the dimensions of consecutive members differ by 1. By the special case, $L^{\prime}(\sigma) \cap \zeta_{1} \subseteq L^{\prime}\left(\zeta_{1}\right)$ and $L^{\prime}\left(\zeta_{1}\right) \cap \zeta_{2} \subseteq L^{\prime}\left(\zeta_{2}\right)$. Since $\zeta_{2} \subseteq \zeta_{1}$ and $\left|L^{\prime}\left(\zeta_{2}\right)\right| \subseteq \zeta_{2}$ and thus, $\zeta_{1} \cap \zeta_{2}=\zeta_{2}$ and $L^{\prime}\left(\zeta_{2}\right) \cap \zeta_{2}=L^{\prime}\left(\zeta_{2}\right)$, we see that $L^{\prime}(\sigma) \cap \zeta_{2} \subseteq L^{\prime}\left(\zeta_{1}\right) \cap \zeta_{2} \subseteq L^{\prime}\left(\zeta_{2}\right)$. Repeating this argument $k$ times we obtain the desired conclusion.

We will now prove the assertion assuming that $\sigma=\left[v_{0}, \ldots, v_{n}\right], c^{*} \in L^{\prime}(\sigma)$ is an $n$-cell and $\zeta$ is the $(n-1)$-dimensional face of $\sigma$ not containing the vertex $v_{l}$, where $0 \leq l \leq n$. By definition, there exists a cell $c \in L(\sigma)$ such that $c^{*} \in L(c)$. If $c$ is the central $n$-simplex $c^{\sigma}$ of $L(\sigma)$, then $c^{*} \cap \zeta=\emptyset \in L(\zeta)$, because $c^{*} \subseteq c=c^{\sigma} \subseteq \operatorname{Int}(\sigma)$ and $\zeta \subseteq \partial \sigma$. Therefore, it suffices to consider the case when $c$ is a peripheral $n$-cell $c=c^{\tau} \oplus d_{\pi}^{\tau} \in L(\sigma)$. There is no loss of generality in assuming that $\tau=\left[v_{0}, \ldots, v_{k}\right]$, where $0 \leq k<n$ and $\pi$ is a permutation of the set $\{k+1, \ldots, n\}$. The proof of Lemma 3.14 contains an argument which shows that $0 \leq l \leq k$ implies $c \cap \zeta=\emptyset$. Since $c^{*} \subseteq c$, it follows that also $c^{*} \cap \zeta=\emptyset$.

Now consider the case when $k+1 \leq l \leq n$. The proof of Lemma 3.14 contains an argument which shows that there is a face $d^{\prime}$ of $d_{\pi}^{\tau}$ and there is a face $\eta$ of $\zeta$ such that $c \cap \zeta=c^{\tau} \oplus d^{\prime}=c^{\tau}+d^{\prime}-w_{0}^{\tau} \in L(\eta)$. Moreover, $\operatorname{dim}\left(c^{\tau} \oplus d^{\prime}\right)=\operatorname{dim} \eta$ and thus, $c^{\tau} \oplus d^{\prime} \in L(\eta)$. On the other hand, $c=$ $c^{\tau} \oplus d_{\pi}^{\tau}$ implies that $L(c)=L\left(c^{\tau}\right) \oplus d_{\pi}^{\tau}$. Therefore, $c^{*} \in L(c)$ is of the form $c^{*}=e \oplus d_{\pi}^{\tau}=e+d_{\pi}^{\tau}-w_{0}^{\tau}$, where $e \in L\left(c^{\tau}\right)$. Since $c^{*} \subseteq c, e \subseteq c^{\tau}$ and $d^{\prime} \leq d_{\pi}^{\tau}$ and thus, $e \cap c^{\tau}=e$ and $d^{\prime} \cap d_{\pi}^{\tau}=d^{\prime}$, we see that $c^{*} \cap \zeta=c^{*} \cap(c \cap \zeta)=$ $\left(e \oplus d_{\pi}^{\tau}\right) \cap\left(c^{\tau} \oplus d^{\prime}\right)=e \oplus d^{\prime} \in L\left(c^{\tau}\right) \oplus d^{\prime}$. Note that $L\left(c^{\tau} \oplus d^{\prime}\right)$ is well defined, because $c^{\tau} \oplus d^{\prime} \in L(\eta)$. Consequently, $L(c \cap \zeta)=L\left(c^{\tau} \oplus d^{\prime}\right)=L\left(c^{\tau}\right) \oplus d^{\prime}$ and thus, $c^{*} \cap \zeta \in L(c \cap \zeta) \subseteq L(c \cap \zeta)$. This and the relation $c \cap \zeta=c^{\tau} \oplus d^{\prime} \in L(\eta)$ show that $c^{*} \cap \zeta \in L^{\prime}(\eta) \subseteq L^{\prime}(\zeta)$.

\section{The SeCond iterated CANONical SUbdivision $N^{\prime}(K)$}

6.1. In this section we first extend the definition of the cellular complex $N\left(\sigma^{\prime}\right)$, associated with an ordered simplex $\sigma^{\prime}$ from $V$ (considered in Section 4 ), to the case of a cellular complex $N\left(\sigma \oplus \sigma^{\prime}\right)$, associated with the direct 
sum $\sigma \oplus \sigma^{\prime}$ of an ordered pair $\left(\sigma, \sigma^{\prime}\right)$ of simplices from $V$, where $\operatorname{dim} \sigma=k$, $\operatorname{dim} \sigma^{\prime}=k^{\prime}$ and $\sigma^{\prime}$ is an ordered simplex. We assume that $\sigma \oplus \sigma^{\prime}$ is well defined, i.e., $\operatorname{Aff}(\sigma) \cap \operatorname{Aff}\left(\sigma^{\prime}\right)$ is a single point $w$ and thus, $\sigma \oplus \sigma^{\prime}=\sigma+\sigma^{\prime}-w$. We will first define the $n$-cells of $N\left(\sigma \oplus \sigma^{\prime}\right)$, where $n=k+k^{\prime}$. Then $N\left(\sigma \oplus \sigma^{\prime}\right)$ will consist of all faces of these $n$-cells. By definition, an $n$-cell of $N\left(\sigma \oplus \sigma^{\prime}\right)$ is a convex polytope of the form $\sigma \oplus c^{\prime}$, where $c^{\prime}$ is a $k^{\prime}$-cell from $N\left(\sigma^{\prime}\right)$.

Clearly, $\operatorname{Aff}\left(c^{\prime}\right)=\operatorname{Aff}\left(\sigma^{\prime}\right)$ and thus, $\operatorname{Aff}(\sigma) \cap \operatorname{Aff}\left(c^{\prime}\right)=\{w\}$. Consequently, $\sigma \oplus c^{\prime}=\sigma+c^{\prime}-w$ is a well-defined $n$-dimensional convex polytope. If we denote by $N_{n}\left(\sigma \oplus \sigma^{\prime}\right)$ the set of all $n$-cells of $N\left(\sigma \oplus \sigma^{\prime}\right)$ and we denote by $N_{k^{\prime}}\left(\sigma^{\prime}\right)$ the set of all $k^{\prime}$-cells of $N\left(\sigma^{\prime}\right)$, we put

$$
N_{n}\left(\sigma \oplus \sigma^{\prime}\right)=\sigma \oplus N_{k^{\prime}}\left(\sigma^{\prime}\right)=\left\{\sigma \oplus c^{\prime} \mid c^{\prime} \in N_{k^{\prime}}\left(\sigma^{\prime}\right)\right\} .
$$

In other words, $N_{n}\left(\sigma \oplus \sigma^{\prime}\right)$ is obtained from $\sigma \oplus \sigma^{\prime}$ by applying the operation $N_{k^{\prime}}$ to the second summand $\sigma^{\prime}$, leaving the first summand $\sigma$ unchanged. If $\sigma$ is a 0 -dimensional simplex $*$, then $\operatorname{Aff}(\sigma)=*$. The reference point of $\sigma \oplus \sigma^{\prime}$ is $*$, because $\operatorname{Aff}(*) \cap \operatorname{Aff}\left(\sigma^{\prime}\right)=*$. Consequently, for $c^{\prime} \in N_{n}\left(\sigma^{\prime}\right)$, the direct sum $* \oplus c^{\prime}=*+c^{\prime}-*=c^{\prime}$ and thus, $N_{n}\left(* \oplus \sigma^{\prime}\right)=\left\{* \oplus c^{\prime} \mid c^{\prime} \in N_{n}\left(\sigma^{\prime}\right)\right\}=$ $\left\{c^{\prime} \mid c^{\prime} \in N_{n}\left(\sigma^{\prime}\right)\right\}=N_{n}\left(\sigma^{\prime}\right)$. Therefore, $N\left(* \oplus \sigma^{\prime}\right)=N\left(\sigma^{\prime}\right)$ and we see that $N\left(\sigma^{\prime}\right)$ is a special case of $N\left(* \oplus \sigma^{\prime}\right)$.

The proof that $N\left(\sigma \oplus \sigma^{\prime}\right)$ is a cellular complex with carrier $\sigma \oplus \sigma^{\prime}$ is analogous to the corresponding proof for $L\left(\sigma \oplus \sigma^{\prime}\right)$.

6.2. We will now apply the above described construction to cells $c$ from the subdivision $L(\sigma)$ of a simplex $\sigma \in K$, described in Section 3. Recall that, for an $n$-simplex $\sigma$, with every $n$-cell $c \in L_{n}(\sigma)$ is associated the ordered pair of simplices $\left(c^{\tau}, d_{\pi}^{\tau}\right)$ such that $c=c^{\tau} \oplus d_{\pi}^{\tau}, \tau \leq \sigma, \operatorname{dim} \tau=k$. Note that $d_{\pi}^{\tau}$ is endowed with a natural ordering, given by the ranks of its vertices. Therefore, $N(c)=N\left(c^{\tau} \oplus d_{\pi}^{\tau}\right)$ is a well-defined cellular complex with carrier $|N(c)|=c$. Its $n$-cells form the set

$$
N_{n}(c)=N_{n}\left(c^{\tau} \oplus d_{\pi}^{\tau}\right)=c^{\tau} \oplus N_{n-k}\left(d_{\pi}^{\tau}\right) .
$$

All other cells are faces of these $n$-cells. The second iterated canonical subdivision $N^{\prime}(\sigma)$ of an $n$-simplex $\sigma \subseteq V$ is defined by the formula

$$
N^{\prime}(\sigma)=\cup_{c \in L_{n}(\sigma)} N(c) .
$$

If $K$ is a simplicial complex in $V$, the second iterated canonical subdivision $N^{\prime}(K)$ is defined by the formula

$$
N^{\prime}(K)=\cup_{\sigma \in K} N^{\prime}(\sigma) .
$$

THEOREM 6.1. If $\sigma$ is a simplex in $V$, then $N^{\prime}(\sigma)$ is a cellular subdivision of $L(\sigma)$ and $\sigma$. If $\zeta<\sigma$, then $N^{\prime}(\zeta) \subseteq N^{\prime}(\sigma)$ and $N^{\prime}(\sigma) \cap \zeta=N^{\prime}(\zeta)$. If $K$ is a simplicial complex in $V$, then $N^{\prime}(K)$ is a cellular subdivision of $L(K)$ and $K$. 
Proof. As in the proof of Theorem 5.1, it readily follows that faces of cells belonging to $N^{\prime}(\sigma)$ also belong to $N^{\prime}(\sigma)$. Therefore, to prove that $N^{\prime}(\sigma)$ is a cellular complex in $V$, it suffices to prove that the intersection $c^{\bullet} \cap c_{1}^{\bullet}$ of two cells $c^{\bullet}, c_{1}^{\bullet}$ from $N^{\prime}(\sigma)$ is a common face of these cells. If $\operatorname{dim} \sigma=n$, there are $n$-cells $c, c_{1} \in L(\sigma)$ such that $c^{\bullet} \in N(c), c_{1}^{\bullet} \in N\left(c_{1}\right)$. As in the proof of Theorem 5.1, there is no loss of generality in assuming that $c^{\bullet}$ and $c_{1}^{\bullet}$ are $n$-cells of $N(c)$ and $N\left(c_{1}\right)$, respectively.

Since $L(\sigma)$ is a cellular complex, the intersection $a=c \cap c_{1}$ is a common face of both cells $c$ and $c_{1}$. We will define a cellular complex $N(a)$ and we will show that

$$
a \cap c^{\bullet} \in N(a), a \cap c_{1}^{\bullet} \in N(a) .
$$

Note that (6.5) implies that the intersection $\left(a \cap c^{\bullet}\right) \cap\left(a \cap c_{1}^{\bullet}\right)$ is a common face of $a \cap c^{\bullet}$ and $a \cap c_{1}^{\bullet}$. Since $c^{\bullet} \subseteq c$ and $c_{1}^{\bullet} \subseteq c_{1}$, it follows that $c^{\bullet} \cap c_{1}^{\bullet} \subseteq c \cap c_{1}=a$ and thus, $c^{\bullet} \cap c_{1}^{\bullet}=\left(a \cap c^{\bullet}\right) \cap\left(a \cap c_{1}^{\bullet}\right)$. Consequently, (6.5) implies

$$
c^{\bullet} \cap c_{1}^{\bullet} \leq a \cap c^{\bullet}, \quad c^{\bullet} \cap c_{1}^{\bullet} \leq a \cap c_{1}^{\bullet} .
$$

The desired assertion that $c^{\bullet} \cap c_{1}^{\bullet}$ is a common face of the cells $c^{\bullet}$ and $c_{1}^{\bullet}$ will follow, if we also show that

$$
a \cap c^{\bullet} \leq c^{\bullet}, \quad a \cap c_{1}^{\bullet} \leq c_{1}^{\bullet} .
$$

We will now define $N(a)$. By Lemma 3.4, applied to $a \leq c=c_{\pi}^{\tau}$, there are faces $c_{a}, d_{a}$ of $a$ such that the vertex scheme of $c_{a}$ is the first row of the vertex scheme of $a$ and $d_{a}$ is the first column of the vertex scheme of $a$. Moreover, $a=c_{a} \oplus d_{a}$ and the reference point $w$ is the only common vertex of $c_{a}$ and $d_{a}$, i.e., $w=w_{j}^{0 \ldots k \pi(k+1) \ldots \pi(k+i)}$. We define $N(a)$ by putting $N(a)=c_{a} \oplus N\left(d_{a}\right)=c_{a}+N\left(d_{a}\right)-w$. Note that the vertices of any column of the vertex scheme of $a$ are ordered by their ranks. Consequently, $N\left(d_{a}\right)$ is well defined.

Let us prove that the cellular complex $N(a)$ is completely determined by $a$, i.e., it does not depend on $c$. This means that, if we have another $n$-cell $c_{1}=$ $c_{\pi^{1}}^{\tau^{1}} \in L(\sigma)$ such that $a \leq c_{1}$ and perform the above construction, the resulting cellular complex $N^{1}(a)$ will coincide with $N(a)$. Indeed, this time Lemma 3.4 will give us faces $c_{a}, d_{a}^{1}$ of $a$ such that $a=c_{a} \oplus d_{a}^{1}=c_{a}+d_{a}^{1}-w^{1}$, the reference point $w^{1}$ being the only common vertex of $c_{a}$ and $d_{a}^{1}$ and the construction will give us the cellular complex $N^{1}(a)=c_{a} \oplus N\left(d_{a}^{1}\right)=c_{a}+N\left(d_{a}^{1}\right)-w^{1}$. Note that the first summand $c_{a}$ is the same as in the case of the $n$-cell $c=c_{\pi}^{\tau}$, because $c_{a}$ is completely determined by $a$ alone. To prove that $N^{1}(a)=N(a)$ we need to prove that

$$
N\left(d_{a}^{1}\right)=N\left(d_{a}\right)+\left(w^{1}-w\right) .
$$

As in the proof of Theorem 5.1, there is an $l, 0 \leq l \leq r$, such that the vertex scheme of $d_{a}^{1}$ is the $j_{l}$-th column of the vertex scheme (3.16) of $a$ and 
the only common vertex of $c_{a}$ and $d_{a}^{1}$ is the vertex $w^{1}=w_{j_{l}}^{0 \ldots k \pi(k+1) \ldots \pi(k+i)}$. Moreover, $w^{1}-w=w_{j_{l}}^{0 \ldots k \pi(k+1) \ldots \pi(k+i)}-w_{j}^{0 \ldots k \pi(k+1) \ldots \pi(k+i)}=w_{j_{l}}^{0 \ldots k}-w_{j}^{0 \ldots k}$. We know by Lemma 3.4, that translation by $w^{1}-w=w_{j_{l}}^{0 \ldots k}-w_{j}^{0 \ldots k}$ is an affine isomorphism between the simplices $d_{a}$ and $d_{a}^{1}$, which preserves the ranks of the vertices and thus, it preserves the ordering of these simplices. The equality (6.8) is an immediate consequence of this fact.

We will now prove that

$$
a \cap N(c)=N(a), \quad a \cap N\left(c_{1}\right)=N(a) .
$$

Since $a=c^{\prime}{ }_{a}+d^{\prime}{ }_{a}-w_{0}^{\tau}, N(c)=c^{\tau}+N\left(d_{\pi}^{\tau}\right)-w_{0}^{\tau}$ and $c^{\prime}{ }_{a} \leq c^{\tau}, d^{\prime}{ }_{a} \leq d_{\pi}^{\tau}$ (notation as in Lemma 3.4), by 2.7, $a \cap N(c)=\left(c^{\prime}{ }_{a} \cap c^{\tau}\right)+\left(d^{\prime}{ }_{a} \cap N\left(d_{\pi}^{\tau}\right)\right)-w_{0}^{\tau}$. By (4.6) (with $\sigma=d_{\pi}^{\tau}$ and $\zeta=d^{\prime}{ }_{a}$ ), one has $d^{\prime}{ }_{a} \cap N\left(d_{\pi}^{\tau}\right)=N\left(d_{a}^{\prime}\right)$. Moreover, $c^{\prime}{ }_{a} \cap c^{\tau}=c^{\prime}{ }_{a}$ and we see that $a \cap N(c)=c^{\prime}{ }_{a}+N\left(d^{\prime}{ }_{a}\right)-w_{0}^{\tau}$. On the other hand, $a=c_{a} \oplus d_{a}$ and $w=w_{j}^{0 \ldots k \pi(k+1) \ldots \pi(k+i)}$ is the corresponding reference point. Therefore, $N(a)=c_{a} \oplus N\left(d_{a}\right)=c_{a}+N\left(d_{a}\right)-w_{j}^{0 \ldots k \pi(k+1) \ldots \pi(k+i)}$. Since $c_{a}=$ $c^{\prime}{ }_{a}+w_{0}^{0 \ldots k \pi(k+1) \ldots \pi(k+i)}-w_{0}^{0 \ldots k}$, one has $N(a)=\left(c^{\prime}{ }_{a}+w_{0}^{0 \ldots k \pi(k+1) \ldots \pi(k+i)}-\right.$ $\left.w_{0}^{0 \ldots k}\right)+N\left(d_{a}\right)-w_{j}^{0 \ldots k \pi(k+1) \ldots \pi(k+i)}=c^{\prime}{ }_{a}+N\left(d_{a}\right)-w_{j}^{\tau}$. Now recall that translation by $w_{j}^{\tau}-w_{0}^{\tau}$ is an order preserving bijection between the vertices of $d^{\prime}{ }_{a}$ and $d_{a}$ (see Lemma 3.4) and thus, it establishes an isomorphism between $N\left(d^{\prime}{ }_{a}\right)$ and $N\left(d_{a}\right)$. Consequently, $N\left(d_{a}\right)=N\left(d^{\prime}{ }_{a}\right)+w_{j}^{\tau}-w_{0}^{\tau}$. It follows that $N(a)=c_{a}+\left(N\left(d^{\prime}{ }_{a}\right)+w_{j}^{\tau}-w_{0}^{\tau}\right)-w_{j}^{0 \ldots k \pi(k+1) \ldots \pi(k+i)}=c_{a}+N\left(d^{\prime}{ }_{a}\right)-$ $w_{0}^{0 \ldots k \pi(k+1) \ldots \pi(k+i)}=c^{\prime}{ }_{a}+N\left(d^{\prime}{ }_{a}\right)-w_{0}^{\tau}=a \cap N(c)$. This establishes the first of the two relations (6.9). The second one follows by symmetry.

If $c^{\bullet} \in N(c)$, then $a \cap c^{\bullet} \in a \cap N(c)$ and by the first of the two relations (6.9), one concludes that $a \cap c^{\bullet} \in N(a)$. Using the second relation of (6.9), we see that $c_{1}^{\bullet} \in N\left(c_{1}\right)$ implies $a \cap c_{1}^{\bullet} \in a \cap N\left(c_{1}\right)=N(a)$. Consequently, both relations (6.5) hold. To complete the proof that $c^{\bullet} \cap c_{1}^{\bullet}$ is a common face of $c^{\bullet}$ and $c_{1}^{\bullet}$, it remains to prove (6.7). Since $N(c)=c^{\tau}+N\left(d_{\pi}^{\tau}\right)-w_{0}^{\tau}$, every $n$-cell $c^{\bullet} \in N(c)$ is of the form $c^{\bullet}=c^{\tau}+d^{\prime}-w_{0}^{\tau}$, where $d^{\prime} \in N\left(d_{\pi}^{\tau}\right)$. Taking into account that $a=c^{\prime}{ }_{a}+d^{\prime}{ }_{a}-w_{0}^{\tau}$, where $c^{\prime}{ }_{a} \leq c^{\tau}$ and $d^{\prime}{ }_{a} \leq d_{\pi}^{\tau}$, we see that $a \cap c^{\bullet}=\left(c^{\tau}+d^{\prime}-w_{0}^{\tau}\right) \cap\left(c^{\prime}{ }_{a}+d^{\prime}{ }_{a}-w_{0}^{\tau}\right)=c^{\prime}{ }_{a}+e-w_{0}^{\tau}$, where $e=d^{\prime} \cap d^{\prime}{ }_{a} \in N\left(d_{\pi}^{\tau}\right) \cap d^{\prime}{ }_{a}$. Since $d_{\pi}^{\tau}$ is a simplex, $d^{\prime} \in N\left(d_{\pi}^{\tau}\right)$ and $d^{\prime}{ }_{a} \leq d_{\pi}^{\tau}$, Lemma 4.4 (with $d_{\pi}^{\tau}, d^{\prime}{ }_{a}$ and $d^{\prime}$ as $\sigma, \zeta$ and $c$ ) shows that $d^{\prime}{ }_{a} \cap N\left(d_{\pi}^{\tau}\right)=N\left(d^{\prime}{ }_{a}\right)$. Since $d^{\prime}{ }_{a} \leq d_{\pi}^{\tau}$, we see that $N\left(d^{\prime}{ }_{a}\right) \subseteq N\left(d_{\pi}^{\tau}\right)$ and thus, $e=d^{\prime} \cap d^{\prime}{ }_{a}$ is a cell of the cellular complex $N\left(d_{\pi}^{\tau}\right)$. However, $d^{\prime}$ is also a cell of $N\left(d_{\pi}^{\tau}\right)$. Therefore, the intersection $e \cap d^{\prime}=e$ of these two cells is a common face of both cells. In particular, $e=d^{\prime} \cap d^{\prime}{ }_{a} \leq d^{\prime}$. Since $c^{\prime}{ }_{a} \leq c^{\tau}$, we conclude that $a \cap c^{\bullet}=$ $c^{\prime}{ }_{a}+e-w_{0}^{\tau} \leq c^{\tau}+d^{\prime}-w_{0}^{\tau}=c^{\bullet}$, which establishes the first of the two relations in (6.7). The second one, follows by symmetry. 
6.3. The cellular complex $N^{\prime}(\sigma)$ is a subdivision of $L(\sigma)$, because $\left|N^{\prime}(c)\right|=c$, for $c \in L(\sigma)$. Since $L(\sigma)$ is a subdivision of $\sigma$, it follows that $N^{\prime}(\sigma)$ is also a subdivision of $\sigma$. The proof that $\zeta<\sigma$ implies $N^{\prime}(\zeta) \subseteq N^{\prime}(\sigma)$ is analogous to the proof of the corresponding relation for $L^{\prime}$ (see Theorem 5.1). The same situation is with the proof of the relation

$$
N^{\prime}(\zeta) \subseteq N^{\prime}(\sigma) \cap \zeta
$$

To prove the opposite inclusion

$$
N^{\prime}(\sigma) \cap \zeta \subseteq N^{\prime}(\zeta),
$$

we proceed as in the proof of Theorem 5.1 and reduce the proof to the case when $\sigma=\left[v_{0}, \ldots, v_{n}\right]$ and $\zeta$ is the $(n-1)$-dimensional face not containing the vertex $v_{l}$, where $0 \leq l \leq n$. If $c^{\bullet} \in N^{\prime}(\sigma)$, then there exists an $n$-cell $c \in L(\sigma)$ such that $c^{\bullet} \in N(c)$. If $c$ is the central $n$-simplex from $L(\sigma)$, then $c^{\bullet} \cap \zeta=\emptyset$, because $c^{\bullet} \subseteq c=c^{\sigma} \subseteq \operatorname{Int}(\sigma)$ and $\zeta \subseteq \partial \sigma$. Therefore, it suffices to consider the case when $c$ is a peripheral $n$-cell $c=c_{\pi}^{\tau} \in L(\sigma)$. There is no loss of generality in assuming that $\tau=\left[v_{0}, \ldots, v_{k}\right]$, where $0 \leq k<n$ and $\pi$ is a permutation of the set $\{k+1, \ldots, n\}$. We saw in the proof of Lemma 3.14 that $0 \leq l \leq k$ implies $c \cap \zeta=\emptyset$. Since $c^{\bullet} \subseteq c$, it follows again that $c^{\bullet} \cap \zeta=\emptyset$. Therefore, it suffices to consider the case when $k+1 \leq l \leq n$.

We saw in the proof of Lemma 3.14 that, for $k+1 \leq l \leq n$, the intersection $c \cap \zeta$ belongs to $L(\zeta)$ and it is of the form $c \cap \zeta=c^{\tau} \oplus d^{\prime}$, where $d^{\prime}$ is a face of $d_{\pi}^{\tau}$. Moreover, $N(c)=N\left(c^{\tau} \oplus d_{\pi}^{\tau}\right)=c^{\tau} \oplus N\left(d_{\pi}^{\tau}\right)$. Since $c^{\bullet} \in N(c)$, there exists a face $c^{\prime} \leq c^{\tau}$ and there exists a cell $e \in N\left(d_{\pi}^{\tau}\right)$ such that $c^{\bullet}=c^{\prime} \oplus e$. Since $c^{\bullet} \subseteq c$, it follows that $c^{\bullet} \cap \zeta=c^{\bullet} \cap(c \cap \zeta)=\left(c^{\prime} \oplus e\right) \cap\left(c^{\tau} \oplus d^{\prime}\right)=\left(c^{\prime} \cap c^{\tau}\right) \oplus\left(e \cap d^{\prime}\right)$. By (4.6), $d^{\prime} \leq d_{\pi}^{\tau}$ and $e \in N\left(d_{\pi}^{\tau}\right)$ imply $N\left(d_{\pi}^{\tau}\right) \cap d^{\prime}=N\left(d^{\prime}\right)$ and thus, $e \cap d^{\prime} \in N\left(d^{\prime}\right)$. On the other hand, $c^{\prime} \cap c^{\tau}=c^{\prime}$ is a face of $c^{\tau}$. Therefore, $c^{\bullet} \cap \zeta=\left(c^{\prime} \cap c^{\tau}\right) \oplus\left(e \cap d^{\prime}\right)=c^{\prime} \oplus\left(e \cap d^{\prime}\right) \in c^{\tau} \oplus N\left(d^{\prime}\right)=N\left(c^{\tau} \oplus d^{\prime}\right)=N(c \cap \zeta)$. Since, $c \cap \zeta \in L(\zeta)$, we obtain the desired conclusion that $c^{\bullet} \cap \zeta \in N^{\prime}(\zeta)$. This completes the proof of (6.11). That $N^{\prime}=N^{\prime}(K)$ is a cellular subdivision of $L(K)$ is an immediate consequence of the equality $N^{\prime}(\sigma) \cap \zeta=N^{\prime}(\zeta)$.

\section{The ISOMORPhism $\vartheta: L^{\prime}(K) \rightarrow N^{\prime}(K)$ AND THE SELFHOMEOMORPHISM $\theta:|K| \rightarrow|K|$}

The main results of this section and of the whole paper are the following theorems, valid for every simplicial complex $K$ in $V$.

THEOREM 7.1. There exists an isomorphism of cellular complexes $\vartheta: L^{\prime}(K) \rightarrow N^{\prime}(K)$, given by a sequence of functions $\vartheta_{k}: L_{k}^{\prime}(K) \rightarrow N_{k}^{\prime}(K)$, $k \in\{0,1, \ldots\}$. For every $n$-simplex $\sigma \in K$, the restrictions $\vartheta_{k} \mid L_{k}^{\prime}(\sigma)$, $0 \leq k \leq n$, form an isomorphism of cellular complexes $\vartheta^{\sigma}: L^{\prime}(\sigma) \rightarrow N^{\prime}(\sigma)$.

Theorem 7.2. There exists a selfhomeomorphism $\theta: P \rightarrow P$ of the carrier $P=|K|$ such that, for every simplex $\sigma \in K, \theta \mid \sigma$ is a selfhomeomorphism 
of $\sigma$. Moreover, for every $k$-cell $c^{*} \in L^{\prime}(\sigma), 0 \leq k \leq n, n=\operatorname{dim} \sigma$, the restriction $\theta \mid c^{*}$ is an affine isomorphism between $c^{*}$ and the $k$-cell $\vartheta_{k}\left(c^{*}\right) \in N^{\prime}(K)$.

Figure 4 shows the complexes $L^{\prime}\left[v_{0}, v_{1}, v_{2}\right]$ and $N^{\prime}\left[v_{0}, v_{1}, v_{2}\right]$. In that case, there is an obvious way of defining an isomorphism $\vartheta$ between the two complexes and a selfhomeomorphism $\theta:\left[v_{0}, v_{1}, v_{2}\right] \rightarrow\left[v_{0}, v_{1}, v_{2}\right]$, whose restrictions to the cells $c^{*}$ of $L^{\prime}\left[v_{0}, v_{1}, v_{2}\right]$ are affine isomorphisms between $c^{*}$ and $\vartheta\left(c^{*}\right)$.
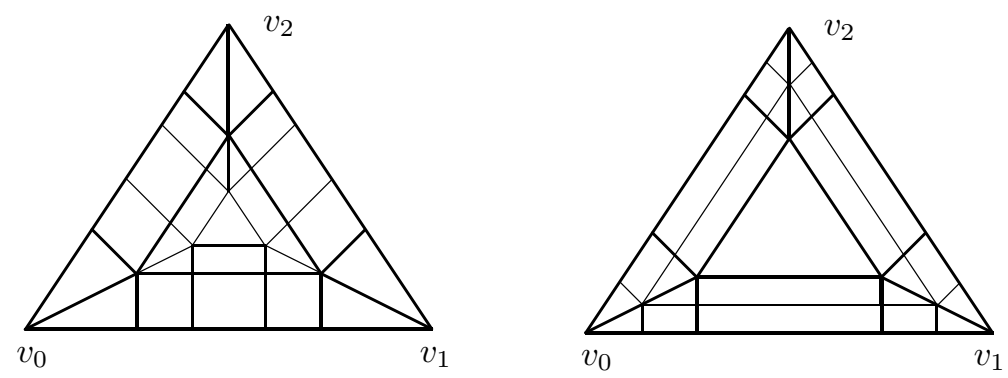

Figure 4. The decompositions $L^{\prime}\left[v_{0}, v_{1}, v_{2}\right]$ and $N^{\prime}\left[v_{0}, v_{1}, v_{2}\right]$

Proof of TheOREMS 7.1 AND 7.2. In the general case, a complete proof of these theorems is rather lengthy and consists of a sequence of steps.

7.1. Step $(i)$. We begin the proof with the following lemma.

Lemma 7.3. For any $n$-simplex $\sigma \in K$, the sets of $n$-cells $L_{n}^{\prime}(\sigma)$ and $N_{n}^{\prime}(\sigma)$ have the same cardinal numbers,

$$
\operatorname{card}\left(L_{n}^{\prime}(\sigma)\right)=\operatorname{card}\left(N_{n}^{\prime}(\sigma)\right)=(n+1) ! \sum_{k=0}^{n} \frac{(n-k+1)}{(k+1) !} .
$$

Proof. If $\sigma=\left[v_{0}, \ldots, v_{n}\right]$, then the $n$-cells of $L(\sigma)$ are of the form $c_{\pi}^{\tau}$, where $\tau$ is a face of $\sigma$ and $\pi$ is a permutation of the set of vertices of $\sigma$, which do not belong to $\tau$. If $\operatorname{dim} \tau=k$, there are $n-k$ such vertices and thus, there are $(n-k)$ ! such permutations. There are $\left(\begin{array}{l}n+1 \\ k+1\end{array}\right) k$-faces $\tau$ of $\sigma$ and therefore, the $n$-cells $c_{\pi}^{\tau}$ with $\operatorname{dim} \tau=k$ yield $\left(\begin{array}{l}n+1 \\ k+1\end{array}\right)(n-k) !=\frac{(n+1) !}{(k+1) !(n-k) !}(n-k) !=\frac{(n+1) !}{(k+1) !}$ $n$-cells of $L(\sigma)$. Since $k$ ranges from 0 to $n$, one concludes that

$$
\operatorname{card}\left(L_{n}(\sigma)\right)=\sum_{k=0}^{n} \frac{(n+1) !}{(k+1) !} .
$$

On the other hand, the $n$-cells of $N(\sigma)$ are of the form $c_{i}^{\sigma}$, where $0 \leq i \leq n$. Therefore,

$$
\operatorname{card}\left(N_{n}(\sigma)\right)=n+1
$$


In order to obtain the $n$-cells of $L_{n}^{\prime}(\sigma)$, every $n$-cell $c_{\pi}^{\tau}=c^{\tau} \oplus d_{\pi}^{\tau}$ of $L_{n}(\sigma)$ with $\operatorname{dim} \tau=k$ is subdivided by replacing the $k$-simplex $c^{\tau}$ by the $k$-cells of $L_{k}\left(c^{\tau}\right)$. Therefore, it yields $\operatorname{card}\left(L_{k}\left(c^{\tau}\right)\right) n$-cells of $L_{n}^{\prime}(\sigma)$. Since there are $\frac{(n+1) !}{(k+1) !} n$-cells $c_{\pi}^{\tau}$ with $\operatorname{dim} \tau=k$, they generate $\frac{(n+1) !}{(k+1) !} \operatorname{card}\left(L_{k}\left(c^{\tau}\right)\right) n$-cells of $L_{n}^{\prime}(\sigma)$. Formula (7.2), applied to the $k$-simplex $c^{\tau}$, shows that

$$
\operatorname{card}\left(L_{k}\left(c^{\tau}\right)\right)=\sum_{l=0}^{n} \frac{(k+1) !}{(l+1) !} .
$$

Since $k$ ranges from 0 to $n$, one concludes that

$$
\operatorname{card}\left(L_{n}^{\prime}(\sigma)\right)=\sum_{k=0}^{n}\left(\frac{(n+1) !}{(k+1) !} \sum_{l=0}^{k} \frac{(k+1) !}{(l+1) !}\right)=\sum_{k=0}^{n} \sum_{l=0}^{k} \frac{(n+1) !}{(l+1) !} .
$$

On the other hand, to obtain the $n$-cells of $N_{n}^{\prime}(\sigma)$, every $n$-cell $c_{\pi}^{\tau}=c^{\tau} \oplus d_{\pi}^{\tau}$ of $L_{n}(\sigma)$ with $\operatorname{dim} \tau=k$ is subdivided by replacing the $(n-k)$-simplex $d_{\pi}^{\tau}$ by the $(n-k)$-cells of $\left.N_{n-k}\left(d_{\pi}^{\tau}\right)\right)$. Therefore, it yields card $\left(N_{n-k}\left(d_{\pi}^{\tau}\right)\right) n$-cells of $N_{n}^{\prime}(\sigma)$. Since there are $\frac{(n+1) !}{(k+1) !} n$-cells $c_{\pi}^{\tau}$ with $\operatorname{dim} \tau=k$, they generate $\frac{(n+1) !}{(k+1) !} \operatorname{card}\left(N_{n-k}\left(d_{\pi}^{\tau}\right)\right) n$-cells of $N_{n}^{\prime}(\sigma)$. Formula (7.3), applied to $d_{\pi}^{\tau}$, shows that

$$
\operatorname{card}\left(N_{n-k}\left(d_{\pi}^{\tau}\right)\right)=n-k+1 .
$$

Since $k$ ranges from 0 to $n$, one concludes that

$$
\operatorname{card}\left(N_{n}^{\prime}(\sigma)\right)=\sum_{k=0}^{n} \frac{(n+1) !}{(k+1) !}(n-k+1)
$$

To see that the right sides in (7.5) and (7.7) are equal, it suffices to change the order of summation in the double sum of (7.5). Indeed,

$$
\sum_{k=0}^{n} \sum_{l=0}^{k} \frac{(n+1) !}{(l+1) !}=\sum_{l=0}^{n} \sum_{k=l}^{n} \frac{(n+1) !}{(l+1) !}=\sum_{l=0}^{n} \frac{(n+1) !}{(l+1) !}(n-l+1) .
$$

ExAmple 7.4. For $\sigma=\left[v_{0}, v_{1}, v_{2}, v_{3}\right], \operatorname{card}\left(L_{3}(\sigma)\right)=41, \operatorname{card}\left(N_{3}(\sigma)\right)=4$ and $\operatorname{card}\left(L_{3}^{\prime}(\sigma)\right)=\operatorname{card}\left(N_{3}^{\prime}(\sigma)\right)=141$.

7.2. Step $(i i)$. For every $n$-simplex $\sigma=\left[v_{0}, \ldots, v_{n}\right] \in K$, we will define a function $\vartheta_{n}^{\sigma}: L_{n}^{\prime}(\sigma) \rightarrow N_{n}^{\prime}(\sigma)$ from the set of $n$-cells of $L^{\prime}(\sigma)$ to the set of $n$-cells of $N^{\prime}(\sigma)$. Recall that every $n$-cell $c^{*} \in L_{n}^{\prime}(\sigma)$ admits an $n$-cell $c \in L_{n}(\sigma)$ such that $c^{*} \in L_{n}(c)$. By the definition of $L(\sigma), c$ is of the form $c=c^{\tau} \oplus d_{\pi}^{\tau}$ and has $w_{0}^{\tau}$ for its reference point. It suffices to define $\vartheta_{n}^{\sigma}\left(c^{*}\right)$ in the case when $\tau=\left[v_{0}, \ldots, v_{k}\right], 0 \leq k \leq n$, and thus, $\pi$ is a permutation of the set $\{k+1, \ldots, n\}$. Let $\alpha^{\tau}: c^{\tau} \rightarrow \tau$ be the affine isomorphism, which maps the vertices $w_{0}^{0 \ldots k}, \ldots, w_{k}^{0 \ldots k}$ of $c^{\tau}$ to the vertices $v_{0}, \ldots, v_{k}$ of $\tau$, respectively. 
Clearly, $L_{k}\left(c^{\tau}\right)$ coincides with $\left(\alpha^{\tau}\right)^{-1}\left(L_{k}(\tau)\right)$ and thus, $L_{n}(c)=L_{k}\left(c^{\tau}\right) \oplus d_{\pi}^{\tau}=$ $\left(\alpha^{\tau}\right)^{-1}\left(L_{k}(\tau)\right) \oplus d_{\pi}^{\tau}$, the reference point being $w_{0}^{\tau}$. Since $c^{*}$ is a cell from $L_{n}(c)$, there exists a $k$-cell $c_{\pi^{\prime}}^{\tau^{\prime}}=c^{\tau^{\prime}} \oplus d_{\pi^{\prime}}^{\tau^{\prime}} \in L_{k}(\tau)$ with reference point $w_{0}^{\tau^{\prime}}$, such that

$$
c^{*}=\left(\alpha^{\tau}\right)^{-1}\left(c_{\pi^{\prime}}^{\tau^{\prime}}\right) \oplus d_{\pi}^{\tau},
$$

the reference point being $w_{0}^{\tau}$. It suffices to define $\vartheta_{n}^{\sigma}\left(c^{*}\right)$ in the case when $\tau^{\prime}=\left[v_{0}, \ldots, v_{k^{\prime}}\right], 0 \leq k^{\prime} \leq k$, and thus, $\pi^{\prime}$ is a permutation of the set $\left\{k^{\prime}+1, \ldots, k\right\}$. In this and similar situations in this section one encounters chains of simplices $\tau^{\prime}<\tau<\sigma$ and cells $c_{\pi^{\prime}}^{\tau^{\prime}}=c^{\tau^{\prime}} \oplus d_{\pi^{\prime}}^{\tau^{\prime}}$. Note that $\pi^{\prime}$ is the permutation of the vertices of $\tau$ (not of $\sigma$ ), which do not belong to $\tau^{\prime}$.

Recall that every $c^{\bullet} \in N_{n}^{\prime}(\sigma)$ admits a cell $c \in L_{n}(\sigma)$ such that $c^{\bullet} \in$ $N_{n}(c)$. Note that $c$ is of the form $c=c^{\bar{\tau}} \oplus d_{\bar{\pi}}$. If $\bar{\tau}=\left[v_{0}, \ldots, v_{\bar{k}}\right], 0 \leq \bar{k} \leq$ $n$, then $c$ has $w_{0}^{\bar{\tau}}$ for its reference point and $\bar{\pi}$ is a permutation of the set $\{\bar{k}+1, \ldots, n\}$. Consider the affine isomorphism $\beta \frac{\bar{\tau}}{\pi}: d_{\bar{\pi}}^{\bar{\tau}} \rightarrow \Delta^{n-\bar{k}}$, which maps the vertices $w_{0}^{0 \ldots \bar{k}}, w_{0}^{0 \ldots \bar{k} \bar{\pi}(\bar{k}+1)}, \ldots, w_{0}^{0 \ldots n}$ of $d_{\bar{\pi}}^{\bar{\tau}}$ to the vertices $e_{0}, \ldots, e_{n-\bar{k}}$ of $\Delta^{n-\bar{k}}$, respectively. Clearly, $N_{n-\bar{k}}\left(d_{\bar{\pi}}^{\bar{\tau}}\right)$ coincides with $\left(\beta_{\bar{\pi}}^{\bar{\tau}}\right)^{-1}\left(N_{n-\bar{k}}\left(\Delta^{n-\bar{k}}\right)\right)$ and thus, $N_{n-\bar{k}}(c)=c^{\bar{\tau}} \oplus N_{n-\bar{k}}\left(d_{\bar{\pi}}^{\bar{\tau}}\right)=c^{\bar{\tau}} \oplus\left(\beta_{\bar{\pi}}^{\bar{\tau}}\right)^{-1}\left(N_{n-\bar{k}}\left(\Delta^{n-\bar{k}}\right)\right)$. Since $c^{\bullet}$ is an $n$-cell from $N(c)$, there exists an $(n-\bar{k})$-cell $P_{i}^{n-\bar{k}}$ in $N_{n-\bar{k}}\left(\Delta^{n-\bar{k}}\right)$, $0 \leq i \leq n-\bar{k}$, such that

$$
c^{\bullet}=c^{\bar{\tau}} \oplus\left(\beta_{\bar{\pi}}^{\bar{\tau}}\right)^{-1}\left(P_{i}^{n-\bar{k}}\right),
$$

the reference point being $w_{0}^{\bar{\tau}}$. Recall that the cells $P_{i}^{n}$ were defined in 4.1.

Given $c^{*}$ as in (7.9), we define $\vartheta_{n}^{\sigma}\left(c^{*}\right)=c^{\bullet} \in N_{n}^{\prime}(\sigma)$ by specifying the data $(\bar{\tau}, \bar{\pi}, i)$ in (7.10) as follows. Put $\bar{\tau}=\tau^{\prime}$ and note that $\bar{k}=\operatorname{dim} \bar{\tau}=$ $\operatorname{dim} \tau^{\prime}=k^{\prime}$. Put $i=k-k^{\prime}$, where $k=\operatorname{dim} \tau$. Define the permutation $\bar{\pi}$ of $\{\bar{k}+1, \ldots, n\}=\left\{k^{\prime}+1, \ldots, k, \ldots, n\right\}$ by putting $\bar{\pi}=\pi^{\prime} \sqcup \pi$, where

$$
\begin{aligned}
& \left(\pi^{\prime} \sqcup \pi\right) \mid\left\{k^{\prime}+1, \ldots, k\right\}=\pi^{\prime}, \\
& \left(\pi^{\prime} \sqcup \pi\right) \mid\{k+1, \ldots, n\}=\pi .
\end{aligned}
$$

Consequently,

$$
c^{\bullet}=\vartheta_{n}^{\sigma}\left(c^{*}\right)=c^{\tau^{\prime}} \oplus\left(\beta_{\pi^{\prime} \sqcup \pi}^{\tau^{\prime}}\right)^{-1}\left(P_{k-k^{\prime}}^{n-k^{\prime}}\right),
$$

the reference point being $w_{0}^{\tau^{\prime}}$.

\subsection{Step $($ iii) .}

LEMMA 7.5. The function $\vartheta_{n}^{\sigma}: L_{n}^{\prime}(\sigma) \rightarrow N_{n}^{\prime}(\sigma)$ is a bijection.

Proof. In view of Lemma 7.3, it suffices to prove that $\vartheta_{n}^{\sigma}: c^{*} \in L_{n}^{\prime}(\sigma) \rightarrow$ $N_{n}^{\prime}(\sigma)$ is a surjection. Indeed, let $c^{\bullet} \in N_{n}^{\prime}(\sigma)$ be as in (7.10). Consider $c^{*} \in L_{n}^{\prime}(\sigma)$ as in (7.9), where $\tau, \tau^{\prime}, \pi, \pi^{\prime}$ are specified as follows. Put $\tau^{\prime}=$ $\bar{\tau}=\left[v_{0}, \ldots, v_{\bar{k}}\right], \tau=\left[v_{0}, \ldots, v_{\bar{k}}, v_{\bar{\pi}(\bar{k}+1)}, \ldots, v_{\bar{\pi}(\bar{k}+i)}\right]$. Note that $k^{\prime}=\bar{k}$ and 
$k=\bar{k}+i$. For $\pi^{\prime}$ take the identity permutation of $\{\bar{\pi}(\bar{k}+1), \ldots, \bar{\pi}(\bar{k}+i)\}$ and for $\pi$ take the identity permutation of $\{\bar{\pi}(\bar{k}+i+1), \ldots, \bar{\pi}(n)\}$. We claim that $\vartheta_{n}^{\sigma}\left(c^{*}\right)=c^{\bullet}$. Indeed, $\tau^{\prime}$ in $(7.11)$ becomes $\bar{\tau}$. The role of $\left\{k^{\prime}+1, \ldots, k\right\}$ in $(7.11)$ is now taken by $\{\bar{\pi}(\bar{k}+1), \ldots, \bar{\pi}(\bar{k}+i)\}$ and the permutation $\pi^{\prime} \sqcup \pi$ of that set reduces to $\pi^{\prime}$, which coincides with the identity. Therefore, the permutation $\pi^{\prime} \sqcup \pi$ of $\{\bar{\pi}(\bar{k}+1), \ldots, \bar{\pi}(\bar{k}+i)\}$ is also the identity. Analogously, the role of $\{k+1, \ldots, n\}$ in $(7.11)$ is now taken by $\{\bar{\pi}(\bar{k}+i+1), \ldots, \bar{\pi}(n)\}$ and the permutation $\pi^{\prime} \sqcup \pi$ of that set reduces to $\pi$, which coincides with the identity. Therefore, $\pi^{\prime} \sqcup \pi$ on $\{\bar{\pi}(\bar{k}+i+1), \ldots, \bar{\pi}(n)\}$ is also the identity. Consequently, the permutation $\pi^{\prime} \sqcup \pi$ of $\{\bar{\pi}(\bar{k}+1), \ldots, \bar{\pi}(n)\}$ is the identity permutation. It follows that the permutation $\pi^{\prime} \sqcup \pi$ of $\{\bar{\pi}(\bar{k}+1), \ldots, \bar{\pi}(\bar{k}+i)\}$ is also the identity. It follows that the sequence $\left(\pi^{\prime} \sqcup \pi\right)(\bar{\pi}(\bar{k}+1)), \ldots,\left(\pi^{\prime} \sqcup\right.$ $\pi)(\bar{\pi}(n))$ coincides with the sequence $(\bar{\pi}(\bar{k}+1)), \ldots,(\bar{\pi}(n))$. Now note that the vertices of $d_{\pi^{\prime} \sqcup \pi}^{\tau^{\prime}}$ form the sequence $w_{0}^{0 \ldots \bar{k}\left(\pi^{\prime} \sqcup \pi\right) \bar{\pi}(\bar{k}+1)}, \ldots, w_{0}^{0 \ldots \bar{k} \ldots\left(\pi^{\prime} \sqcup \pi\right) \bar{\pi}(n)}$, which coincides with the sequence $w_{0}^{0 \ldots \bar{k} \bar{\pi}(\bar{k}+1)}, \ldots, w_{0}^{0 \ldots \bar{k} \ldots \bar{\pi}(n)}$ of the vertices of $d_{\bar{\pi}}^{\bar{\tau}}$. Consequently, $\beta_{\pi^{\prime} \sqcup \pi}^{\tau^{\prime}}=\beta_{\bar{\pi}}^{\bar{\tau}}$. Finally, $P_{k-k^{\prime}}^{n-k^{\prime}}$ in (7.11) becomes $P_{k-\bar{k}}^{n-\bar{k}}=$ $P_{i}^{n-\bar{k}}$ and we conclude that indeed, $\vartheta_{n}^{\sigma}\left(c^{*}\right)=c^{\bullet}$.

7.4. Step (iv). For an $n$-simplex $\sigma \in K$ and for $c^{*} \in L_{n}^{\prime}(\sigma), c^{\bullet}=\vartheta_{n}^{\sigma}\left(c^{*}\right) \in$ $N_{n}^{\prime}(\sigma)$, we will now define an affine isomorphism $\theta_{c^{*}}^{\sigma}: c^{*} \rightarrow c^{\bullet}$. By definition, $\theta_{c^{*}}^{\sigma}$ is the composition $\theta_{c^{*}}^{\sigma}=\theta_{c^{*}}^{\sigma 2} \theta_{c^{*}}^{\sigma 1}$ of two affine isomorphisms $\theta_{c^{*}}^{\sigma 1}$ and $\theta_{c^{*}}^{\sigma 2}$. The first one $\theta_{c^{*}}^{\sigma 1}: c^{*}=\left(\alpha^{\tau}\right)^{-1}\left(c_{\pi^{\prime}}^{\tau^{\prime}}\right) \oplus d_{\pi}^{\tau} \rightarrow c_{\pi^{\prime}}^{\tau^{\prime}} \oplus d_{\pi}^{\tau}=\left(c^{\tau^{\prime}} \oplus d_{\pi^{\prime}}^{\tau^{\prime}}\right) \oplus d_{\pi}^{\tau}$ is defined by putting

$$
\theta_{c^{*}}^{\sigma 1}=\left(\alpha^{\tau} \mid\left(\alpha^{\tau}\right)^{-1}\left(c_{\pi^{\prime}}^{\tau^{\prime}}\right)\right) \oplus 1,
$$

the reference point being $w_{0}^{\tau}$. This is a well-defined affine isomorphism, because the mapping $\alpha^{\tau} \mid\left(\alpha^{\tau}\right)^{-1}\left(c_{\pi^{\prime}}^{\tau^{\prime}}\right):\left(\alpha^{\tau}\right)^{-1}\left(c_{\pi^{\prime}}^{\tau^{\prime}}\right) \rightarrow c_{\pi^{\prime}}^{\tau^{\prime}}$ is an affine isomorphism and the direct sum $c_{\pi^{\prime}}^{\tau^{\prime}} \oplus d_{\pi}^{\tau}$ is well defined and has $w_{0}^{\tau}$ for its reference point. The first assertion is a consequence of the fact that $\alpha^{\tau}: c^{\tau} \rightarrow \tau$ is an affine isomorphism and $c_{\pi^{\prime}}^{\tau^{\prime}} \subseteq \tau$. To verify the second assertion, it suffices to see that $\operatorname{Aff}\left(c_{\pi^{\prime}}^{\tau^{\prime}}\right) \cap \operatorname{Aff}\left(d_{\pi}^{\tau}\right)=\left\{w_{0}^{\tau}\right\}$. Indeed, $\operatorname{Aff}\left(c_{\pi^{\prime}}^{\tau^{\prime}}\right) \subseteq \operatorname{Aff}(\tau)$ implies $\operatorname{Aff}\left(c_{\pi^{\prime}}^{\tau^{\prime}}\right) \cap \operatorname{Aff}\left(d_{\pi}^{\tau}\right) \subseteq \operatorname{Aff}(\tau) \cap \operatorname{Aff}\left(d_{\pi}^{\tau}\right)=\left\{w_{0}^{\tau}\right\}$. Moreover, $w_{0}^{\tau}$ is a vertex of $c_{\pi^{\prime}}^{\tau^{\prime}}$ and of $d_{\pi}^{\tau}$ and thus, $\left\{w_{0}^{\tau}\right\} \subseteq \operatorname{Aff}\left(c_{\pi^{\prime}}^{\tau^{\prime}}\right) \cap \operatorname{Aff}\left(d_{\pi}^{\tau}\right)$.

Before describing $\theta_{c^{*}}^{\sigma 2}$, let us show that

$$
\left(c^{\tau^{\prime}} \oplus d_{\pi^{\prime}}^{\tau^{\prime}}\right) \oplus d_{\pi}^{\tau}=c^{\tau^{\prime}} \oplus\left(d_{\pi^{\prime}}^{\tau^{\prime}} \oplus d_{\pi}^{\tau}\right)=c^{\tau^{\prime}}+d_{\pi^{\prime}}^{\tau^{\prime}}+d_{\pi}^{\tau}-w_{0}^{\tau^{\prime}}-w_{0}^{\tau} .
$$

Formula (7.15) is obtained by applying the following elementary fact.

If $A, B, C$ are sets in a vector space $V$ and the direct sums $A \oplus B,(A \oplus B) \oplus$ $C, B \oplus C$ and $A \oplus(B \oplus C)$ are well defined, then $(A \oplus B) \oplus C=A \oplus(B \oplus C)$ and one can use the notation $A \oplus B \oplus C$. Moreover, if $A \oplus B$ and $B \oplus C$ have the points $w$ and $w^{\prime}$ for their reference points, then $A \oplus B \oplus C=A+B+C-w-w^{\prime}$. 
In order to be able to apply this fact, we first note that the direct sum $c^{\tau^{\prime}} \oplus d_{\pi^{\prime}}^{\tau^{\prime}}$ is well defined and its reference point is $w_{0}^{\tau^{\prime}}$. Furthermore, $d_{\pi^{\prime}}^{\tau^{\prime}} \oplus d_{\pi}^{\tau}$ is well defined and its reference point is $w_{0}^{\tau}$, i.e., $\operatorname{Aff}\left(d_{\pi^{\prime}}^{\tau^{\prime}}\right) \cap \operatorname{Aff}\left(d_{\pi}^{\tau}\right)=\left\{w_{0}^{\tau}\right\}$. Indeed, $d_{\pi^{\prime}}^{\tau^{\prime}} \subseteq c_{\pi^{\prime}}^{\tau^{\prime}} \subseteq c^{\tau}$ implies $\operatorname{Aff}\left(d_{\pi^{\prime}}^{\tau^{\prime}}\right) \cap \operatorname{Aff}\left(d_{\pi}^{\tau}\right) \subseteq \operatorname{Aff}\left(c^{\tau}\right) \cap \operatorname{Aff}\left(d_{\pi}^{\tau}\right)=\left\{w_{0}^{\tau}\right\}$ and $w_{0}^{\tau} \in d_{\pi^{\prime}}^{\tau^{\prime}} \cap d_{\pi}^{\tau}$. Moreover, let us show that $c^{\tau^{\prime}} \oplus\left(d_{\pi^{\prime}}^{\tau^{\prime}} \oplus d_{\pi}^{\tau}\right)$ is also well defined and its reference point is $w_{0}^{\tau^{\prime}}$, i.e., $\operatorname{Aff}\left(c^{\tau^{\prime}}\right) \cap \operatorname{Aff}\left(d_{\pi^{\prime}}^{\tau^{\prime}} \oplus d_{\pi}^{\tau}\right)=\left\{w_{0}^{\tau^{\prime}}\right\}$. Consider the permutation $\pi^{\prime} \sqcup \pi$ of $\left\{k^{\prime}+1, \ldots, n\right\}$, defined by (7.11) and (7.12) and consider the $n$-cell $c_{\pi^{\prime} \sqcup \pi}^{\tau^{\prime}}=c^{\tau^{\prime}} \oplus d_{\pi^{\prime} \sqcup \pi}^{\tau^{\prime}} \in L(\sigma)$. By the definition of the $n$-cells from $L(\sigma)$, we know that $\operatorname{Aff}\left(c^{\tau^{\prime}}\right) \cap \operatorname{Aff}\left(d_{\pi^{\prime} \sqcup \pi}^{\tau^{\prime}}\right)=\left\{w_{0}^{\tau^{\prime}}\right\}$. To complete the proof of the assertion, it suffices to show that $\operatorname{Aff}\left(d_{\pi^{\prime} \sqcup \pi}^{\tau^{\prime}}\right)=\operatorname{Aff}\left(d_{\pi^{\prime}}^{\tau^{\prime}} \oplus d_{\pi}^{\tau}\right)$. First note that the union of the sets of vertices of $d_{\pi^{\prime}}^{\tau^{\prime}}$ and $d_{\pi}^{\tau}$ coincides with the set of vertices of $d_{\pi^{\prime} \sqcup \pi \pi}^{\tau^{\prime}}$. Therefore, $d_{\pi^{\prime}}^{\tau^{\prime}}$ and $d_{\pi}^{\tau}$ are faces of $d_{\pi^{\prime} \sqcup \pi}^{\tau^{\prime}}$. Since $d_{\pi^{\prime}}^{\tau^{\prime}} \oplus d_{\pi}^{\tau}=d_{\pi^{\prime}}^{\tau^{\prime}}+d_{\pi}^{\tau}-w_{0}^{\tau^{\prime}}$ and $w_{0}^{\tau^{\prime}} \in d_{\pi^{\prime} \sqcup \pi}^{\tau^{\prime}} \subseteq \operatorname{Aff}\left(d_{\pi^{\prime} \sqcup \pi}^{\tau^{\prime}}\right)$, it follows that $d_{\pi^{\prime}}^{\tau^{\prime}} \oplus d_{\pi}^{\tau} \subseteq \operatorname{Aff}\left(d_{\pi^{\prime} \sqcup \pi}^{\tau^{\prime}}\right)$, hence also $\operatorname{Aff}\left(d_{\pi^{\prime}}^{\tau^{\prime}} \oplus d_{\pi}^{\tau}\right) \subseteq \operatorname{Aff}\left(d_{\pi^{\prime} \sqcup \pi}^{\tau^{\prime}}\right)$. On the other hand, every vertex $w$ of $d_{\pi^{\prime} \sqcup \pi}^{\tau^{\prime}}$ is a vertex of $d_{\pi^{\prime}}^{\tau^{\prime}} \subseteq d_{\pi^{\prime}}^{\tau^{\prime}} \oplus d_{\pi}^{\tau}$ or a vertex of $d_{\pi}^{\tau} \subseteq d_{\pi^{\prime}}^{\tau^{\prime}} \oplus d_{\pi}^{\tau}$. Since $\operatorname{Aff}\left(d_{\pi^{\prime} \sqcup \pi}^{\tau^{\prime}}\right)$ coincides with the affine hull of the set of vertices of $d_{\pi^{\prime} \sqcup \pi}^{\tau^{\prime}}$, one concludes that $\operatorname{Aff}\left(d_{\pi^{\prime} \sqcup \pi}^{\tau^{\prime}}\right) \subseteq \operatorname{Aff}\left(d_{\pi^{\prime}}^{\tau^{\prime}} \oplus d_{\pi}^{\tau}\right)$.

Consider the affine isomorphism $\gamma^{c^{*}}: d_{\pi^{\prime}}^{\tau^{\prime}} \rightarrow Q_{k-k^{\prime}}^{k-k^{\prime}}$, which maps the vertices $w_{0}^{0 \ldots k^{\prime}}, w_{0}^{0 \ldots k^{\prime} \pi^{\prime}\left(k^{\prime}+1\right)}, \ldots, w_{0}^{0 \ldots k}$ of $d_{\pi^{\prime}}^{\tau^{\prime}}$ to the vertices $b^{0 k-k^{\prime}}, b^{1 k-k^{\prime}}, \ldots$, $b^{k-k^{\prime} k-k^{\prime}}$ of $Q_{k-k^{\prime}}^{k-k^{\prime}}$, respectively. Similarly, consider the affine isomorphism $\delta^{c^{*}}: d_{\pi}^{\tau} \rightarrow R_{k-k^{\prime}}^{n-k}$, which maps the vertices $w_{0}^{0 \ldots k}, w_{0}^{0 \ldots k \pi(k+1)}, \ldots, w_{0}^{0 \ldots n}$ of $d_{\pi}^{\tau}$ to the vertices $b^{k-k^{\prime} k-k^{\prime}}, b^{k-k^{\prime} k-k^{\prime}+1}, \ldots, b^{k-k^{\prime} n-k^{\prime}}$ of $R_{k-k^{\prime}}^{n-k}$, respectively. These isomorphisms determine the affine isomorphism $\gamma^{c^{*}} \oplus \delta^{c^{*}}: d_{\pi^{\prime}}^{\tau^{\prime}} \oplus d_{\pi}^{\tau} \rightarrow$ $Q_{k-k^{\prime}}^{k-k^{\prime}} \oplus R_{k-k^{\prime}}^{n-k}=P_{k-k^{\prime}}^{n-k^{\prime}}$ (recall that the cells $Q_{i}^{i}$ and $R_{i}^{n-i}$ were defined in

4.1.) Now note that $\beta_{\pi^{\prime} \sqcup \pi}^{\tau^{\prime}}: d_{\pi^{\prime} \sqcup \pi}^{\tau^{\prime}} \rightarrow \Delta^{n-k^{\prime}}$ is an affine isomorphism and $P_{k-k^{\prime}}^{n-k^{\prime}} \subseteq \Delta^{n-k^{\prime}}$. Therefore,

$$
\varepsilon^{c^{*}}=\left(\beta_{\pi^{\prime} \sqcup \pi}^{\tau^{\prime}}\right)^{-1} \mid P_{k-k^{\prime}}^{n-k^{\prime}}
$$

is an affine isomorphism $\varepsilon^{c^{*}}: P_{k-k^{\prime}}^{n-k^{\prime}} \rightarrow\left(\beta_{\pi^{\prime} \sqcup \pi}^{\tau^{\prime}}\right)^{-1}\left(P_{k-k^{\prime}}^{n-k^{\prime}}\right)$. We now define $\theta_{c^{*}}^{\sigma 2}: c^{\tau^{\prime}} \oplus\left(d_{\pi^{\prime}}^{\tau^{\prime}} \oplus d_{\pi}^{\tau}\right) \rightarrow c^{\tau^{\prime}} \oplus\left(\beta_{\pi^{\prime} \sqcup \pi}^{\tau^{\prime}}\right)^{-1}\left(P_{k-k^{\prime}}^{n-k^{\prime}}\right)=c^{\bullet}$, by putting

$$
\theta_{c^{*}}^{\sigma 2}=1 \oplus \varepsilon^{c^{*}}\left(\gamma^{c^{*}} \oplus \delta^{c^{*}}\right) .
$$

The isomorphisms $\theta^{\sigma 1} c^{*}$ and $\theta^{\sigma 2} c^{*}$ can be composed, because of (7.15).

Since $\theta_{c^{*}}^{\sigma}$ is an affine mapping, it is completely determined by its values at the vertices $w$ of the $n$-cell $c^{*}$. We will now determine explicitly these values. Since the direct sum $\left(\alpha^{\tau}\right)^{-1}\left(c_{\pi^{\prime}}^{\tau^{\prime}}\right) \oplus d_{\pi}^{\tau}$ has $w_{0}^{\tau}$ for its reference point, the vertices of $c^{*}=\left(\alpha^{\tau}\right)^{-1}\left(c_{\pi^{\prime}}^{\tau^{\prime}}\right) \oplus d_{\pi}^{\tau}$ are the points $\left(\alpha^{\tau}\right)^{-1}\left(w^{\prime}\right) \oplus w^{\prime \prime}=\left(\alpha^{\tau}\right)^{-1}\left(w^{\prime}\right)+w^{\prime \prime}-$ $w_{0}^{\tau}$, where $w^{\prime}$ ranges over the set of vertices of $c_{\pi^{\prime}}^{\tau^{\prime}}$ and $w^{\prime \prime}$ ranges over the set of vertices of $d_{\pi}^{\tau}$. By $(3.11), w^{\prime}$ is of the form $w_{s}^{0 \ldots k^{\prime} \pi^{\prime}\left(k^{\prime}+1\right) \ldots \pi^{\prime}\left(k^{\prime}+r\right)}$, where 
$0 \leq s \leq k^{\prime}, 0 \leq r \leq k-k^{\prime}$ and $w^{\prime \prime}$ is of the form $w_{0}^{0 \ldots k \pi(k+1) \ldots \pi(k+l)}$, where $0 \leq l \leq n-k$. Consequently, the vertices $w$ of $c^{*}$ are of the form

$$
w=\left(\alpha^{\tau}\right)^{-1}\left(w_{s}^{0 \ldots k^{\prime} \pi^{\prime}\left(k^{\prime}+1\right) \ldots \pi^{\prime}\left(k^{\prime}+r\right)}\right) \oplus w_{0}^{0 \ldots k \pi(k+1) \ldots \pi(k+l)},
$$

where $0 \leq s \leq k^{\prime}, 0 \leq r \leq k-k^{\prime}$ and $0 \leq l \leq n-k$, and the reference point is $w_{0}^{\tau}$. By $(7.14), \theta_{c^{*}}^{\sigma 1}(w)=w_{s}^{0 \ldots k^{\prime} \pi^{\prime}\left(k^{\prime}+1\right) \ldots \pi^{\prime}\left(k^{\prime}+r\right)}+w_{0}^{0 \ldots k \pi(k+1) \ldots \pi(k+l)}-w_{0}^{\tau}$. Since $w_{s}^{0 \ldots k^{\prime} \pi^{\prime}\left(k^{\prime}+1\right) \ldots \pi^{\prime}\left(k^{\prime}+r\right)}=w_{0}^{0 \ldots k^{\prime} \pi^{\prime}\left(k^{\prime}+1\right) \ldots \pi^{\prime}\left(k^{\prime}+r\right)}+w_{s}^{0 \ldots k^{\prime}}-w_{0}^{0 \ldots k^{\prime}}$, one concludes that

$$
\begin{aligned}
& \theta_{c^{*}}^{\sigma 1}(w)=w_{s}^{0 \ldots k^{\prime}}-w_{0}^{0 \ldots k^{\prime}}+ \\
& \left(w_{0}^{0 \ldots k^{\prime} \pi^{\prime}\left(k^{\prime}+1\right) \ldots \pi^{\prime}\left(k^{\prime}+r\right)}+w_{0}^{0 \ldots k \pi(k+1) \ldots \pi(k+l)}-w_{0}^{0 \ldots k}\right) .
\end{aligned}
$$

The expression in the parenthesis equals

$$
w_{0}^{0 \ldots k^{\prime} \pi^{\prime}\left(k^{\prime}+1\right) \ldots \pi^{\prime}\left(k^{\prime}+r\right)} \oplus w_{0}^{0 \ldots k \pi(k+1) \ldots \pi(k+l)} \in d_{\pi^{\prime}}^{\tau^{\prime}} \oplus d_{\pi}^{\tau},
$$

because $w_{0}^{0 \ldots k^{\prime} \pi^{\prime}\left(k^{\prime}+1\right) \ldots \pi^{\prime}\left(k^{\prime}+r\right)} \in d_{\pi^{\prime}}^{\tau^{\prime}}, w_{0}^{0 \ldots k \pi(k+1) \ldots \pi(k+l)} \in d_{\pi}^{\tau}$ and $d_{\pi^{\prime}}^{\tau^{\prime}} \oplus d_{\pi}^{\tau}$ has $w_{0}^{\tau}$ for its reference point. Since $w_{s}^{0 \ldots k^{\prime}} \in c^{\tau^{\prime}}$ and $c^{\tau^{\prime}} \oplus\left(d_{\pi^{\prime}}^{\tau^{\prime}} \oplus d_{\pi}^{\tau}\right)$ have $w_{0}^{\tau^{\prime}}$ for their reference point, one sees that the right side of (7.19) equals

$$
w_{s}^{0 \ldots k^{\prime}} \oplus\left(w_{0}^{0 \ldots k^{\prime} \pi^{\prime}\left(k^{\prime}+1\right) \ldots \pi^{\prime}\left(k^{\prime}+r\right)} \oplus w_{0}^{0 \ldots k \pi(k+1) \ldots \pi(k+l)}\right) \in c^{\tau^{\prime}} \oplus\left(d_{\pi^{\prime}}^{\tau^{\prime}} \oplus d_{\pi}^{\tau}\right) .
$$

Now note that

$$
\begin{aligned}
& \left(\gamma^{c^{*}} \oplus \delta^{c^{*}}\right)\left(w_{0}^{0 \ldots k^{\prime} \pi^{\prime}\left(k^{\prime}+1\right) \ldots \pi^{\prime}\left(k^{\prime}+r\right)} \oplus w_{0}^{0 \ldots k \pi(k+1) \ldots \pi(k+l)}\right)= \\
& \gamma^{c^{*}}\left(w_{0}^{0 \ldots k^{\prime} \pi^{\prime}\left(k^{\prime}+1\right) \ldots \pi^{\prime}\left(k^{\prime}+r\right)}\right) \oplus \delta^{c^{*}}\left(w_{0}^{0 \ldots k \pi(k+1) \ldots \pi(k+l)}\right) .
\end{aligned}
$$

However,

$$
\begin{gathered}
\gamma^{c^{*}}\left(w_{0}^{0 \ldots k^{\prime} \pi^{\prime}\left(k^{\prime}+1\right) \ldots \pi^{\prime}\left(k^{\prime}+r\right)}\right)=b^{r k-k^{\prime}} \\
\delta^{c^{*}}\left(w_{0}^{0 \ldots k \pi(k+1) \ldots \pi(k+l)}\right)=b^{k-k^{\prime}, k-k^{\prime}+l}
\end{gathered}
$$

and thus,

$$
\begin{aligned}
& \left(\gamma^{c^{*}} \oplus \delta^{c^{*}}\right)\left(w_{0}^{0 \ldots k^{\prime} \pi^{\prime}\left(k^{\prime}+1\right) \ldots \pi^{\prime}\left(k^{\prime}+r\right)} \oplus w_{0}^{0 \ldots k \pi(k+1) \ldots \pi(k+l)}\right)= \\
& b^{r k-k^{\prime}} \oplus b^{k-k^{\prime}, k-k^{\prime}+l} .
\end{aligned}
$$

Since $0 \leq r \leq k-k^{\prime},\left(\pi^{\prime} \sqcup \pi\right)\left(k^{\prime}+1\right)=\pi^{\prime}\left(k^{\prime}+1\right), \ldots,\left(\pi^{\prime} \sqcup \pi\right)\left(k^{\prime}+\right.$ $r)=\pi^{\prime}\left(k^{\prime}+r\right)$, one concludes that $\beta_{\pi^{\prime} \sqcup \pi}^{\tau^{\prime}}\left(w_{0}^{0 \ldots k^{\prime} \pi^{\prime}\left(k^{\prime}+1\right) \ldots \pi^{\prime}\left(k^{\prime}+r\right)}\right)=e_{r}$. In particular, $\beta_{\pi^{\prime} \sqcup \pi}^{\tau^{\prime}}\left(w_{0}^{0 \ldots k}\right)=e_{k-k^{\prime}}$. Therefore, $\beta_{\pi^{\prime} \sqcup \pi}^{\tau^{\prime}}\left(\frac{1}{2}\left(w_{0}^{0 \ldots k^{\prime} \pi^{\prime}\left(k^{\prime}+1\right) \ldots \pi^{\prime}\left(k^{\prime}+r\right)}+\right.\right.$ $\left.\left.w_{0}^{0 \ldots k}\right)\right)=\frac{1}{2}\left(e_{r}+e_{k-k^{\prime}}\right)=b^{r, k-k^{\prime}}$. Similarly, since $0 \leq l \leq n-k$, we see that $\left(\pi^{\prime} \sqcup \pi\right)(k+1)=\pi(k+1), \ldots,\left(\pi^{\prime} \sqcup \pi\right)(k+l)=\pi(k+l)$ and thus, $\beta_{\pi^{\prime} \sqcup \pi}^{\tau^{\prime}}\left(w_{0}^{0 \ldots k \pi(k+1) \ldots \pi(k+l)}\right)=e_{k-k^{\prime}+l}$. Consequently,

$$
\beta_{\pi^{\prime} \sqcup \pi}^{\tau^{\prime}}\left(\frac{1}{2}\left(w_{0}^{0 \ldots k \pi(k+1) \ldots \pi(k+l)}+w_{0}^{0 \ldots k}\right)\right)=\frac{1}{2}\left(e_{k-k^{\prime}+l}+e_{k-k^{\prime}}\right)=b^{k-k^{\prime}+l, k-k^{\prime}} .
$$


It follows that

$$
\begin{aligned}
& \left(\beta_{\pi^{\prime} \sqcup \pi \pi}^{\tau^{\prime}}\right)^{-1}\left(b^{r k-k^{\prime}} \oplus b^{k-k^{\prime}, k-k^{\prime}+l}\right)= \\
& \frac{1}{2}\left(w_{0}^{0 \ldots k^{\prime} \pi^{\prime}\left(k^{\prime}+1\right) \ldots \pi^{\prime}\left(k^{\prime}+r\right)}+w_{0}^{0 \ldots k \pi(k+1) \ldots \pi(k+l)}\right) .
\end{aligned}
$$

Now (7.16) and (7.25) show that

$$
\begin{aligned}
& \varepsilon^{c^{*}}\left(\gamma^{c^{*}} \oplus \delta^{c^{*}}\right)\left(w_{0}^{0 \ldots k^{\prime} \pi^{\prime}\left(k^{\prime}+1\right) \ldots \pi^{\prime}\left(k^{\prime}+r\right)} \oplus w_{0}^{0 \ldots k \pi(k+1) \ldots \pi(k+l)}\right)= \\
& \frac{1}{2}\left(w_{0}^{0 \ldots k^{\prime} \pi^{\prime}\left(k^{\prime}+1\right) \ldots \pi^{\prime}\left(k^{\prime}+r\right)}+w_{0}^{0 \ldots k \pi(k+1) \ldots \pi(k+l)}\right) .
\end{aligned}
$$

Finally, (7.17) and (7.26) show that $\theta_{c^{*}}^{\sigma}$ maps the vertex $w$ of $c^{*}$, given by (7.18), to the vertex

$$
\theta_{c^{*}}^{\sigma}(w)=w_{s}^{0 \ldots k^{\prime}} \oplus \frac{1}{2}\left(w_{0}^{0 \ldots k^{\prime} \pi^{\prime}\left(k^{\prime}+1\right) \ldots \pi^{\prime}\left(k^{\prime}+r\right)}+w_{0}^{0 \ldots k \pi(k+1) \ldots \pi(k+l)}\right),
$$

where the reference point is $w_{0}^{\tau^{\prime}}$.

7.5. Step $(v)$. Let $\sigma \in K$ be an $n$-simplex. We define a mapping $\theta^{\sigma}: \sigma \rightarrow$ $\sigma$, by putting

$$
\theta^{\sigma} \mid c^{*}=\theta_{c^{*}}^{\sigma},
$$

for every $n$-cell $c^{*} \in L_{n}^{\prime}(\sigma)$. Note that $\theta_{c^{*}}^{\sigma}$ maps $c^{*}$ to $\vartheta_{n}^{\sigma}\left(c^{*}\right) \in N_{n}^{\prime}(\sigma)$ and thus, $\theta_{c^{*}}^{\sigma}\left(c^{*}\right) \subseteq \vartheta_{n}^{\sigma}\left(c^{*}\right) \subseteq \sigma$. To see that the mapping $\theta^{\sigma}$ is well defined, it suffices to prove the following lemma.

Lemma 7.6. Let $\sigma$ be an n-simplex from $K$. For any two $n$-cells $c^{*}, c_{1}^{*} \in$ $L^{\prime}(\sigma)$, the restrictions of $\theta_{c^{*}}^{\sigma}$ and $\theta_{c_{1}^{*}}^{\sigma}$ to the intersection $c^{*} \cap c_{1}^{*}$ coincide, i.e.,

$$
\theta_{c^{*}}^{\sigma}\left|\left(c^{*} \cap c_{1}^{*}\right)=\theta_{c_{1}^{*}}^{\sigma}\right|\left(c^{*} \cap c_{1}^{*}\right) .
$$

Proof. It suffices to prove (7.29) in the special case when $c^{*} \cap c_{1}^{*}$ is a common $(n-1)$-face of the $n$-cells $c^{*}$ and $c_{1}^{*}$. Indeed, assume that $c^{*} \cap c_{1}^{*}=e^{*}$ is an $l$-cell, $0 \leq l<n-1$. Note that all $n$-cells $C$ of $L^{\prime}(\sigma)$ which contain $e^{*}$, together with their faces, form a cellular complex, whose carrier is an $n$-cell. Since $n$-cells are manifolds with boundary, there exists a sequence of $n$-cells $c^{*}=C_{0}, \ldots, C_{k}=c_{1}^{*}$ of $L^{\prime}(\sigma)$ such that the intersections $C_{i} \cap C_{i+1}$ of consecutive members of the sequence are common $(n-1)$-faces of $C_{i}$ and $C_{i+1}$. By the special case of the lemma, $\theta_{C_{i}}^{\sigma}\left|\left(C_{i} \cap C_{i+1}\right)=\theta_{C_{i+1}}^{\sigma}\right|\left(C_{i} \cap C_{i+1}\right)$. Since $e^{*} \subseteq C_{i} \cap C_{i+1}$, one concludes that $\theta_{c^{*}}^{\sigma}\left|e^{*}=\ldots=\theta_{C_{i}}^{\sigma}\right| e^{*}=\theta_{C_{i+1}}^{\sigma} \mid e^{*}=$ $\ldots=\theta_{c_{1}^{*}}^{\sigma} \mid e^{*}$.

We now consider the special case when $c^{*}, c_{1}^{*}$ are different $n$-cells, $c^{*} \cap c_{1}^{*}=$ $e^{*}$ and $\operatorname{dim} e^{*}=n-1$. To prove that $\theta_{c^{*}}^{\sigma}\left|e^{*}=\theta_{c_{1}^{*}}^{\sigma}\right| e^{*}$, it suffices to prove that

$$
\theta_{c^{*}}^{\sigma}(w)=\theta_{c_{1}^{*}}^{\sigma}(w),
$$

for all vertices $w$ of the cell $e^{*}$. There is no loss of generality in assuming that $\tau=\left[v_{0}, \ldots, v_{k}\right], 0 \leq k \leq n, \tau^{\prime}=\left[v_{0}, \ldots, v_{k^{\prime}}\right], 0 \leq k^{\prime} \leq k, \pi^{\prime}=\iota^{\prime}$ is the identity permutation of the set $\left\{k^{\prime}+1, \ldots, k\right\}, \pi=\iota$ is the identity 
permutation of the set $\{k+1, \ldots, n\}$ and thus, $\pi^{\prime} \sqcup \pi=\eta$ is the identity permutation of $\left\{k^{\prime}+1, \ldots, n\right\}$. Then (7.9) assumes the form

$$
c^{*}=\left(\alpha^{\tau}\right)^{-1}\left(c_{\iota^{\prime}}^{\tau^{\prime}}\right) \oplus d_{\iota}^{\tau},
$$

the direct sum having $w_{0}^{0 \ldots k}$ for its reference point. Consequently, the vertices $w$ of $c^{*}$ are of the form

$$
w=\left(\alpha^{\tau}\right)^{-1}\left(w_{s}^{0 \ldots k^{\prime}+r}\right) \oplus w_{0}^{0 \ldots k+l},
$$

where $0 \leq s \leq k^{\prime}, 0 \leq r \leq k-k^{\prime}, 0 \leq l \leq n-k$ and the reference point is $w_{0}^{0 \ldots k}$. Furthermore, (7.27) shows that

$$
\theta_{c^{*}}^{\sigma}(w)=w_{s}^{0 \ldots k^{\prime}} \oplus \frac{1}{2}\left(w_{0}^{0 \ldots k^{\prime}+r}+w_{0}^{0 \ldots k+l}\right),
$$

the direct sum having $w_{0}^{0 \ldots . . k^{\prime}}$ for its reference point. Given the $n$-cell $c^{*}$ of $L^{\prime}(\sigma)$ and an $(n-1)$-face $e^{*}$ of $c^{*}$, either there is no $n$-cell $c_{1}^{*} \neq c^{*}$ with $e^{*}<c_{1}^{*}$ or there is a unique $n$-cell $c_{1}^{*}$ having this property ( $\sigma$ is an $n$-manifold). We will now consider all $(n-1)$-faces $e^{*}$ of $c^{*}$, we will determine the corresponding $n$-cells $c_{1}^{*}$ (if they exist) and we will verify (7.30). Concerning $e^{*}$, two cases are possible: Case I, when

$$
e^{*}=\left(\alpha^{\tau}\right)^{-1}\left(e^{\prime}\right)+d_{\iota}^{\tau}-w_{0}^{\tau}
$$

and $e^{\prime}$ is a $(k-1)$-face of $c_{\iota^{\prime}}^{\tau^{\prime}}=c^{\tau^{\prime}} \oplus d_{\iota^{\prime}}^{\tau^{\prime}}, k \geq 1$, and Case II, when

$$
e^{*}=\left(\alpha^{\tau}\right)^{-1}\left(c_{\iota^{\prime}}^{\tau^{\prime}}\right)+d-w_{0}^{\tau},
$$

and $d$ is a $(n-k-1)$-face of $d_{\iota}^{\tau}, n \geq k+1$.

7.5.1. Case I. We distinguish two subcases, I.1, when $e^{\prime}=c^{\prime}+d_{t^{\prime}}^{\tau^{\prime}}-w_{0}^{0 \ldots k^{\prime}}$, where $c^{\prime}$ is a $\left(k^{\prime}-1\right)$-face of $c^{\tau^{\prime}}$ and I.2, when $e^{\prime}=c^{\tau^{\prime}}+d^{\prime}-w^{0 \ldots k^{\prime}}$, where $d^{\prime}$ is a $\left(k-k^{\prime}-1\right)$-face of $d_{L^{\prime}}^{\tau^{\prime}}$.

Subcase I.1. This subcase can appear only when $k^{\prime} \geq 1$. For symmetry reasons, it suffices to consider the situation, when $c^{\prime}=\left[w_{0}^{0 \ldots k^{\prime}}, \ldots, w_{k^{\prime}}^{0} \ldots k_{1}^{\prime}\right]$. Then the vertex scheme of $e^{\prime}$ is obtained from the vertex scheme (3.11) for $c_{\iota^{\prime}}^{\tau^{\prime}}$, by deleting the last column. This is the scheme

$$
\begin{array}{lll}
w_{0}^{0 \ldots k^{\prime}} & \ldots & w_{k^{\prime}-1}^{0 \ldots k^{\prime}} \\
\ldots & \ldots & \ldots \\
w_{0}^{0 \ldots k} & \ldots & w_{k^{\prime}-1}^{0 \ldots k}
\end{array}
$$

and thus, the vertices of $e^{\prime}$ are of the form $\left(\alpha^{\tau}\right)^{-1}\left(w^{\prime}\right)$, where $w^{\prime}=w_{s}^{0 \ldots k^{\prime}+r}$, $0 \leq s \leq k^{\prime}-1,0 \leq r \leq k-k^{\prime}$. It follows that the vertices $w$ of $e^{*}$ are given by (7.32), where $0 \leq s \leq k^{\prime}-1,0 \leq r \leq k-k^{\prime}$ and $0 \leq l \leq n-k$. Moreover, the $\theta_{c^{*}}^{\sigma}$-images of the vertices $w$ of $e^{*}$ are given by (7.33).

Adding to the scheme (7.36) an initial row $w_{0}^{0 \ldots k^{\prime}-1} \ldots w_{k^{\prime}-1}^{0 \ldots k^{\prime}-1}$ produces the vertex scheme of the $k$-cell $c_{\iota_{1}^{\prime}}^{\tau_{1}^{\prime}}=c^{\tau_{1}^{\prime}} \oplus d_{\iota_{1}^{\prime}}^{\tau_{1}^{\prime}} \in L_{k}(\tau)$, where $\tau_{1}^{\prime}=\left[v_{0}, \ldots, v_{k_{1}^{\prime}}\right]$, $k_{1}^{\prime}=k^{\prime}-1$ and $\iota_{1}^{\prime}$ is the identity permutation of $\left\{k_{1}^{\prime}+1, \ldots, k\right\}=\left\{k^{\prime}, \ldots, k\right\}$. 
Clearly, $e^{\prime}$ is a $(k-1)$-face of $c_{\iota_{1}^{\prime}}^{\tau_{1}^{\prime}}$ and thus, $e^{*}$ is also an $(n-1)$-face of the $n$-cell $\left(\alpha^{\tau}\right)^{-1}\left(c_{\iota_{1}^{\prime}}^{\tau_{1}^{\prime}}\right) \oplus d_{\iota}^{\tau} \in L^{\prime}(\sigma)$. Since $e^{*}$ can be the face of only two $n$-cells, we conclude that

$$
c_{1}^{*}=\left(\alpha^{\tau}\right)^{-1}\left(c_{\iota_{1}^{\prime}}^{\tau_{1}^{\prime}}\right) \oplus d_{\iota}^{\tau} .
$$

To prove that $\theta_{c^{*}}\left|e^{*}=\theta_{c_{1}^{*}}\right| e^{*}$, it suffices to see that the mappings $\theta_{c^{*}}^{\sigma}$ and $\theta_{c_{1}^{*}}^{\sigma}$ assume the same values at the vertices $w$ of $e^{*}$. The vertex $w=\left(\alpha^{\tau}\right)^{-1}\left(w_{s}^{0 \ldots k^{\prime}+r}\right) \oplus w_{0}^{0 \ldots k+l}$ of $e^{*}$ can be written in the form $w=$ $\left(\alpha^{\tau}\right)^{-1}\left(w_{s}^{0 \ldots k_{1}^{\prime}+r+1}\right) \oplus w_{0}^{0 \ldots k+l}$. Therefore, (7.33) for $c_{1}^{*}$, shows that $\theta_{c_{1}^{*}}^{\sigma}(w)=$ $w_{s}^{0 \ldots k_{1}^{\prime}} \oplus \frac{1}{2}\left(w_{0}^{0 \ldots k_{1}^{\prime}+r+1}+w_{0}^{0 \ldots k+l}\right)$, the reference point being $w_{0}^{0 \ldots k_{1}^{\prime}}=w_{0}^{0 \ldots k^{\prime}-1}$. Consequently, $\theta_{c_{1}^{*}}^{\sigma}(w)=w_{s}^{0 \ldots k^{\prime}-1}+\frac{1}{2}\left(w_{0}^{0 \ldots k^{\prime}+r}+w_{0}^{0 \ldots k+l}\right)-w_{0}^{0 \ldots k^{\prime}-1}$. On the other hand, by (7.33) for $c^{*}$, one has $\theta_{c^{*}}^{\sigma}(w)=w_{s}^{0 \ldots k^{\prime}}+\frac{1}{2}\left(w_{0}^{0 \ldots k^{\prime}+r}+w_{0}^{0 \ldots k+l}\right)-$ $w_{0}^{0 \ldots k^{\prime}}$ and thus, $\theta_{c_{1}^{*}}^{\sigma}(w)=\theta_{c^{*}}^{\sigma}(w)$, because $w_{s}^{0 \ldots k^{\prime}}-w_{0}^{0 \ldots k^{\prime}}=w_{s}^{0 \ldots k^{\prime}-1}-w_{0}^{0 \ldots k^{\prime}-1}$ (see (3.14) for $k=k^{\prime}, i=s$ and $k=k^{\prime}-1, i=s$ ).

Subcase I.2. In this subcase $e^{\prime}=c^{\tau^{\prime}}+d^{\prime}-w^{0 \ldots k^{\prime}}$, where $d^{\prime}$ is a $\left(k-k^{\prime}-1\right)$ face of $d_{\iota^{\prime}}^{\tau^{\prime}}=\left[w_{0}^{0 \ldots k^{\prime}}, \ldots, w_{0}^{0 \ldots k}\right]$. We distinguish three subsubcases, I.2.1, when $d^{\prime}=\left[w_{0}^{0 \ldots k^{\prime}+1}, \ldots, w_{0}^{0 \ldots k}\right], 0 \leq k^{\prime}<k$, Subsubcase I.2.2, when $d^{\prime}=$ $\left[w_{0}^{0 \ldots k^{\prime}}, \ldots, w_{0}^{0 \ldots k^{\prime}+i-1}, w_{0}^{0 \ldots k^{\prime}+i+1}, \ldots, w_{0}^{0 \ldots k}\right], 0<i<k-k^{\prime}$, and Subsubcase I.2.3, when $d^{\prime}=\left[w_{0}^{0 \ldots k^{\prime}}, \ldots, w_{0}^{0 \ldots k-1}\right], 0 \leq k^{\prime}<k$.

Subsubcase I.2.1. This time $d^{\prime}=\left[w_{0}^{0 \ldots k^{\prime}+1}, \ldots, w_{0}^{0 \ldots k}\right], 0 \leq k^{\prime}<k$. Therefore, if one omits the first row in the vertex scheme (3.11) for $c_{\iota^{\prime}}^{\tau^{\prime}}$, one obtains the vertex scheme of $e^{\prime}=c^{\tau^{\prime}}+d^{\prime}-w^{0 \ldots k^{\prime}}$, which has the form

$$
\begin{array}{lll}
w_{0}^{0 \ldots k^{\prime}+1} & \ldots & w_{k^{\prime}}^{0 \ldots k^{\prime}+1} \\
\ldots & \ldots & \ldots . \\
w_{0}^{0 \ldots k} & \ldots & w_{k^{\prime}}^{0 \ldots k} .
\end{array}
$$

Adding to that scheme a terminal column $w_{k^{\prime}+1}^{0 \ldots k^{\prime}+1} \ldots w_{k^{\prime}+1}^{0 \ldots k}$ produces the vertex scheme of the $k$-cell $c_{\iota_{1}^{\prime}}^{\tau_{1}^{\prime}} \in L_{k}(\tau)$, where this time $\tau_{1}^{\prime}=\left[v_{0}, \ldots, v_{k_{1}^{\prime}}\right]$, $k_{1}^{\prime}=k^{\prime}+1$ and $\iota_{1}^{\prime}$ is the identity permutation of $\left\{k_{1}^{\prime}+1, \ldots, k\right\}=\left\{k^{\prime}+2, \ldots, k\right\}$ (if $k^{\prime}+1=k, \iota_{1}^{\prime}$ is empty). Since $e^{\prime}$ is a $(k-1)$-face of $c_{\iota_{1}^{\prime}}^{\tau_{1}^{\prime}}$, it follows that $e^{*}$ is also an $(n-1)$-face of the $n$-cell $c_{1}^{*} \in L^{\prime}(\sigma)$, which has the form (7.37). The vertex $w=\left(\alpha^{\tau}\right)^{-1}\left(w_{s}^{0 \ldots k^{\prime}+r}\right) \oplus w_{0}^{0 \ldots k+l}$ of $c^{*}$ is a vertex of $e^{*}$ provided $0 \leq s \leq k^{\prime}, 1 \leq r \leq k-k^{\prime}, 0 \leq l \leq n-k$. Note that $w$ can be written in the form $w=\left(\alpha^{\tau}\right)^{-1}\left(w_{s}^{0 \ldots k_{1}^{\prime}+r-1}\right) \oplus w_{0}^{0 \ldots k+l}$, the reference point being $w_{0}^{0 \ldots k}$. By formula (7.33), applied to $c_{1}^{*}$, one concludes that $\theta_{c_{1}^{*}}(w)=$ $w_{s}^{0 \ldots k_{1}^{\prime}} \oplus \frac{1}{2}\left(w_{0}^{0 \ldots k_{1}^{\prime}+r-1}+w_{0}^{0 \ldots k+l}\right)=w_{s}^{0 \ldots k^{\prime}+1} \oplus \frac{1}{2}\left(w_{0}^{0 \ldots k^{\prime}+r}+w_{0}^{0 \ldots k+l}\right)$, the reference point being $w_{0}^{0 \ldots k_{1}^{\prime}}=w_{0}^{0 \ldots k^{\prime}+1}$. It follows that $\theta_{c_{1}^{*}}(w)=w_{s}^{0 \ldots k^{\prime}+1}+$ 
$\frac{1}{2}\left(w^{0 \ldots k^{\prime}+r}+w_{0}^{0 \ldots k+l}\right)-w_{0}^{0 \ldots k^{\prime}+1}$. Comparing this with (7.33) for $c^{*}$, one concludes that $\theta_{c_{1}^{*}}(w)=\theta_{c^{*}}(w)$, because $w_{s}^{0 \ldots k^{\prime}}-w_{0}^{0 \ldots k^{\prime}}=w_{s}^{0 \ldots k^{\prime}+1}-w_{0}^{0 \ldots k^{\prime}+1}$.

Subsubcase I.2.2. This time

$$
d^{\prime}=\left[w_{0}^{0 \ldots k^{\prime}}, \ldots, w_{0}^{0 \ldots k^{\prime}+i-1}, w_{0}^{0 \ldots k^{\prime}+i+1}, \ldots, w_{0}^{0 \ldots k}\right], 0<i<k-k^{\prime},
$$

and the vertex scheme of $e^{\prime}=c^{\tau^{\prime}}+d^{\prime}-w^{0 \ldots k^{\prime}}$ is obtained from the vertex scheme (3.11) for $c_{\iota^{\prime}}^{\tau^{\prime}}$ by omitting the $i$-th row $w_{0}^{0 \ldots \ldots k^{\prime}+i} \ldots w_{k^{\prime}}^{0 \ldots k^{\prime}+i}$. Let $\pi^{\prime}$ be the permutation of the set $\left\{k^{\prime}+1, \ldots, k\right\}$, which interchanges $k^{\prime}+i$ and $k^{\prime}+i+1$ and otherwise coincides with the identity mapping $\eta^{\prime}$ of $\left\{k^{\prime}+1, \ldots, k\right\}$. Then the vertex scheme of $c_{\pi^{\prime}}^{\tau^{\prime}}$ is

$$
\begin{array}{lll}
w_{0}^{0 \ldots \ldots k^{\prime}} & \ldots & w_{k^{\prime}}^{0} \ldots k^{\prime} \\
\ldots & \ldots & \ldots \\
w_{0}^{0 \ldots \ldots k^{\prime}+i-1} & \ldots & w_{k^{\prime}}^{0 \ldots \ldots k^{\prime}+i-1} \\
w_{0}^{0 \ldots} \ldots k^{\prime}+i-1 k^{\prime}+i+1 & \ldots & w_{k^{\prime}}^{0} \ldots k^{\prime}+i-1 k^{\prime}+i+1 \\
w_{0}^{0 \ldots \ldots k^{\prime}+i+1} & \ldots & w_{k^{\prime}}^{0 \ldots} \ldots k^{\prime}+i+1 \\
w_{0}^{0 \ldots k^{\prime}+i+2} & \ldots & w_{k^{\prime}}^{0 \ldots} k^{\prime}+i+2 \\
\ldots & \ldots & \ldots \\
w_{0}^{0 \ldots k} & \ldots & w_{k^{\prime}}^{0 \ldots \ldots k} .
\end{array}
$$

Note that the omission of the $i$-th row $w_{0}^{0 \ldots k^{\prime}+i-1 k^{\prime}+i+1} \ldots w_{k^{\prime}}^{0 \ldots k^{\prime}+i-1 k^{\prime}+i+1}$ of (7.39) yields the vertex scheme of $e^{\prime}$ and thus, $e^{\prime}$ is also a face of the $n$-cell

$$
c_{1}^{*}=\left(\alpha^{\tau}\right)^{-1}\left(c_{\pi^{\prime}}^{\tau^{\prime}}\right) \oplus d_{\iota}^{\tau} .
$$

Note that the vertex $w=\left(\alpha^{\tau}\right)^{-1}\left(w_{s}^{0 \ldots k^{\prime}+r}\right) \oplus w_{0}^{0 \ldots k+l}$ of $c^{*}$ is a vertex of $e^{*}$ provided $0 \leq s \leq k^{\prime}, 0 \leq r \leq k-k^{\prime}, r \neq i$ and $0 \leq l \leq n-k$. In that case $w=\left(\alpha^{\tau}\right)^{-1}\left(w_{s}^{0 \ldots k^{\prime} \pi^{\prime}\left(k^{\prime}+1\right) \ldots \pi^{\prime}\left(k^{\prime}+r\right)}\right) \oplus w_{0}^{0 \ldots k+l}$, because $\left\{\pi^{\prime}\left(k^{\prime}+\right.\right.$ $\left.1), \ldots, \pi^{\prime}\left(k^{\prime}+r\right)\right\}=\left\{k^{\prime}+1, \ldots, k^{\prime}+r\right\}$, for $0 \leq r \neq i$. E.g., for $r=i+1$ one has $\left\{\pi^{\prime}\left(k^{\prime}+1\right), \ldots, \pi^{\prime}\left(k^{\prime}+i-1\right), \pi^{\prime}\left(k^{\prime}+i\right), \pi^{\prime}\left(k^{\prime}+i+1\right)\right\}=\left\{k^{\prime}+\right.$ $\left.1, \ldots, k^{\prime}+i-1, k^{\prime}+i+1, k^{\prime}+i\right\}=\left\{k^{\prime}+1, \ldots, k^{\prime}+i+1\right\}$. Now (7.27) shows that, for $r \neq i, \theta_{c_{1}^{*}}(w)=w_{s}^{0 \ldots k^{\prime}} \oplus \frac{1}{2}\left(w_{0}^{0 \ldots k^{\prime} \pi^{\prime}\left(k^{\prime}+1\right) \ldots \pi^{\prime}\left(k^{\prime}+r\right)}+w_{0}^{0 \ldots k+l}\right)=$ $w_{s}^{0 \ldots k^{\prime}} \oplus \frac{1}{2}\left(w_{0}^{0 \ldots \ldots k^{\prime}+r}+w_{0}^{0 \ldots k+l}\right)=\theta_{c^{*}}(w)$.

Subsubcase I.2.3. This time $d^{\prime}=\left[w_{0}^{0 \ldots k^{\prime}}, \ldots, w_{0}^{0 \ldots k-1}\right], 0 \leq k^{\prime}<k$, and the vertex scheme of the $(k-1)$-cell $e^{\prime}=c^{\tau^{\prime}}+d^{\prime}-w_{0}^{0 \ldots k^{\prime}} \in L(\tau)$ is obtained from the vertex scheme (3.11), for the $k$-cell $c_{\iota^{\prime}}^{\tau^{\prime}}$, by omitting the last row. Consequently, the vertices of $e^{\prime}$ are of the form $w^{\prime}=w_{s}^{0 \ldots k^{\prime}+r}$, where $0 \leq s \leq$ $k^{\prime}, 0 \leq r \leq k-k^{\prime}-1$. The vertices $w$ of $e^{*}=\left(\alpha^{\tau}\right)^{-1}\left(e^{\prime}\right)+d_{\iota}^{\tau}-w_{0}^{\tau}$ are of the form (7.32), where $0 \leq s \leq k^{\prime}, 0 \leq r \leq k-k^{\prime}-1$ and $0 \leq l \leq n-k$. Furthermore, their $\theta_{c^{*}}$-images are given by (7.33). Now note that the vertex scheme of $e^{\prime}$ coincides with the vertex scheme of the $(k-1)$-cell $c_{\iota_{1}^{\prime}}^{\tau^{\prime}}$, where $\iota_{1}^{\prime}$ is the identity permutation of the set $\left\{k^{\prime}+1, \ldots, k-1\right\}$ so that $c_{\iota_{1}^{\prime}}^{\tau^{\prime}}=e^{\prime}$. 
Let $\tau_{1}=\left[v_{0}, \ldots, v_{k-1}\right]$ and let $\iota_{1}$ be the identity permutation of the set $\{k, \ldots, n\}$. Then the direct sum $\left(\alpha^{\tau_{1}}\right)^{-1}\left(c_{\iota_{1}^{\prime}}^{\tau^{\prime}}\right) \oplus d_{\iota_{1}}^{\tau_{1}}$ is well defined and its reference point is $w_{0}^{0 \ldots k-1}$. Let us now show that

$$
c_{1}^{*}=\left(\alpha^{\tau_{1}}\right)^{-1}\left(c_{\iota_{1}^{\prime}}^{\tau^{\prime}}\right) \oplus d_{\iota_{1}}^{\tau_{1}} .
$$

It suffices to show that $e^{*}$ is a face of the right side of (7.41). First note that $\tau_{1}<\tau$ and

$$
\left(\alpha^{\tau}\right)^{-1} \mid \tau_{1}=\left(\alpha^{\tau_{1}}\right)^{-1}+w_{0}^{0 \ldots k}-w_{0}^{0 \ldots k-1} .
$$

This formula holds, because both sides are affine mappings defined on $\tau_{1}$, which at each of the vertices $v_{0}, \ldots, v_{k-1}$ of $\tau_{1}$ assume the same values. Indeed, $\left(\alpha^{\tau}\right)^{-1}\left(v_{i}\right)=w_{i}^{0 \ldots k}$ and $\left(\alpha^{\tau_{1}}\right)^{-1}\left(v_{i}\right)+w_{0}^{0 \ldots k}-w_{0}^{0 \ldots k-1}=w_{i}^{0 \ldots k-1}+w_{0}^{0 \ldots k}-$ $w_{0}^{0 \ldots k-1}=w_{i}^{0 \ldots k}$. Since $e^{\prime} \subseteq \tau_{1},(7.42)$ implies that $\left(\alpha^{\tau}\right)^{-1}\left(e^{\prime}\right)=\left(\alpha^{\tau_{1}}\right)^{-1}\left(e^{\prime}\right)+$ $w_{0}^{0 \ldots k}-w_{0}^{0 \ldots k-1}$ and thus,

$$
e^{*}=\left(\alpha^{\tau_{1}}\right)^{-1}\left(e^{\prime}\right)+d_{\iota}^{\tau}-w_{0}^{0 \ldots k-1} .
$$

Now note that $d_{\iota}^{\tau}=\left[w^{0 \ldots k}, \ldots, w_{0}^{0 \ldots n}\right]<\left[w^{0 \ldots k-1}, \ldots, w_{0}^{0 \ldots n}\right]=d_{\iota_{1}}^{\tau_{1}}$. Since the direct sum in (7.41) has $w_{0}^{0 \ldots k-1}$ for its reference point, we see that it equals $\left(\alpha^{\tau_{1}}\right)^{-1}\left(e^{\prime}\right)+d_{\iota_{1}}^{\tau_{1}}-w_{0}^{0 \ldots k-1}$. A comparison with (7.43) shows that $e^{*}$ is indeed a face of the right side of (7.41).

Now consider a vertex $w=\left(\alpha^{\tau}\right)^{-1}\left(w_{s}^{0 \ldots k^{\prime}+r}\right) \oplus w_{0}^{0 \ldots k+l}$ of $e^{*}$ and note that $0 \leq r \leq k-k^{\prime}-1$. Since $\left(\alpha^{\tau}\right)^{-1}\left(c_{\iota^{\prime}}^{\tau^{\prime}}\right) \oplus d_{\iota}^{\tau}$ has $w_{0}^{0 \ldots k}$ for its reference point, we see that $w=\left(\alpha^{\tau}\right)^{-1}\left(w_{s}^{0 \ldots k^{\prime}+r}\right)+w_{0}^{0 \ldots k+l}-w_{0}^{0 \ldots k}$. Also note that $0 \leq r \leq k-k^{\prime}-1$ implies $w_{s}^{0 \ldots k^{\prime}+r} \in \tau_{1}$ and thus, (7.42) shows that $\left(\alpha^{\tau}\right)^{-1}\left(w_{s}^{0 \ldots k^{\prime}+r}\right)=\left(\alpha^{\tau_{1}}\right)^{-1}\left(w_{s}^{0 \ldots k^{\prime}+r}\right)+w_{0}^{0 \ldots k}-w_{0}^{0 \ldots k-1}$. Consequently, the vertex $w$ can also be written in the form $w=\left(\alpha^{\tau_{1}}\right)^{-1}\left(w_{s}^{0 \ldots k^{\prime}+r}\right)+w_{0}^{0 \ldots k+l}-$ $w_{0}^{0 \ldots k-1}=\left(\alpha^{\tau_{1}}\right)^{-1}\left(w_{s}^{0 \ldots k^{\prime}+r}\right)+w_{0}^{0 \ldots k_{1}+l+1}-w_{0}^{0 \ldots k_{1}} \in\left(\alpha^{\tau_{1}}\right)^{-1}\left(c_{\iota_{1}^{\prime}}^{\tau^{\prime}}\right) \oplus d_{\iota_{1}}^{\tau_{1}}$, where $k_{1}=k-1$. Now (7.33) for $c_{1}^{*}$ shows that

$$
\theta_{c_{1}^{*}}(w)=w_{s}^{0 \ldots k^{\prime}} \oplus \frac{1}{2}\left(w_{0}^{0 \ldots k^{\prime}+r}+w_{0}^{0 \ldots k_{1}+l+1}\right) .
$$

However, this equals $\theta_{c^{*}}(w)=w_{s}^{0 \ldots k^{\prime}} \oplus \frac{1}{2}\left(w_{0}^{0 \ldots k^{\prime}+r}+w_{0}^{0 \ldots k+l}\right)$, because $k_{1}+$ $l+1=k+l$ and both direct sums have $w_{0}^{0 \ldots k^{\prime}}$ for their reference point.

7.5.2. Case II. In this case $e^{*}$ is given by (7.35), where $d$ is an $(n-k-1)$-face of $d_{\iota}^{\tau}=\left[w_{0}^{0 \ldots k}, \ldots, w_{0}^{0 \ldots n}\right], 0 \leq k<n$. We distinguish three subcases, II.1, when $d=\left[w_{0}^{0 \ldots k+1}, \ldots, w_{0}^{0 \ldots n}\right]$, II.2, when $d=$ $\left[w_{0}^{0 \ldots k}, \ldots, w_{0}^{0 \ldots k+i-1}, w_{0}^{0 \ldots k+i+1}, \ldots, w_{0}^{0 \ldots n}\right], 0<i<n-k$, and II.3, when $d=\left[w_{0}^{0 \ldots k}, \ldots, w_{0}^{0 \ldots n-1}\right]$.

Subcase II.1. Put $\tau_{1}=\left[v_{0}, \ldots, v_{k+1}\right]$ and let $\iota_{1}^{\prime}$ be the identity permutation of $\left\{k^{\prime}+1, \ldots, k+1\right\}$. Then $c_{\iota_{1}^{\prime}}^{\tau^{\prime}}=c^{\tau^{\prime}} \oplus d_{\iota_{1}^{\prime}}^{\tau^{\prime}}$ is a $(k+1)$-cell from $L\left(\tau_{1}\right)$. Note that $d=\left[w_{0}^{0 \ldots k+1}, \ldots, w_{0}^{0 \ldots n}\right]=d_{\iota_{1}}^{\tau_{1}}$, where $\iota_{1}$ is the identity permutation of 
$\{k+2, \ldots, n\}$. Consequently, $c_{\iota_{1}}^{\tau_{1}}=c^{\tau_{1}} \oplus d_{\iota_{1}}^{\tau_{1}}=c^{\tau_{1}} \oplus d$ is well defined and $w_{0}^{0 \ldots k+1}$ is its reference point. Since $\operatorname{dim} c_{\iota_{1}^{\prime}}^{\tau^{\prime}}=\operatorname{dim} \tau_{1}=k+1$ and $c_{\iota_{1}^{\prime}}^{\tau^{\prime}} \subseteq \tau_{1}$, one has $\operatorname{Aff}\left(c_{\iota_{1}^{\prime}}^{\tau^{\prime}}\right)=\operatorname{Aff}\left(\tau_{1}\right)$ and thus also $\operatorname{Aff}\left(\alpha^{\tau_{1}}\right)^{-1}\left(c_{\iota_{1}^{\prime}}^{\tau^{\prime}}\right)=\operatorname{Aff}\left(c^{\tau_{1}}\right)$. It follows that $\operatorname{Aff}\left(\alpha^{\tau_{1}}\right)^{-1}\left(c_{\iota_{1}^{\prime}}^{\tau^{\prime}}\right) \cap \operatorname{Aff}(d)=\operatorname{Aff}\left(c^{\tau_{1}}\right) \cap \operatorname{Aff}(d)=\left\{w_{0}^{0 \ldots k+1}\right\}$. This proves that $\left(\alpha^{\tau_{1}}\right)^{-1}\left(c_{\iota_{1}^{\prime}}^{\tau^{\prime}}\right) \oplus d$ is a well-defined $n$-cell of $L^{\prime}(\sigma)$, also having $w_{0}^{0 \ldots k+1}$ for its reference point. Let us now show that

$$
c_{1}^{*}=\left(\alpha^{\tau_{1}}\right)^{-1}\left(c_{\iota_{1}^{\prime}}^{\tau^{\prime}}\right) \oplus d .
$$

To prove this assertion, it suffices to prove that $e^{*}$ is a face of the right side of (7.45). First note that $\tau<\tau_{1}$ and

$$
\left(\alpha^{\tau_{1}}\right)^{-1} \mid \tau=\left(\alpha^{\tau}\right)^{-1}+w_{0}^{0 \ldots k+1}-w_{0}^{0 \ldots k} .
$$

This formula holds because both sides are affine mappings defined on $\tau$, which at each of the vertices $v_{0}, \ldots, v_{k}$ of $\tau$ assume the same values. Indeed, for $0 \leq i \leq k$, one has $\left(\alpha^{\tau_{1}}\right)^{-1}\left(v_{i}\right)=w_{i}^{0 \ldots k+1}$ and $\left(\alpha^{\tau}\right)^{-1}\left(v_{i}\right)+w_{0}^{0 \ldots k+1}-w_{0}^{0 \ldots k}=$ $w_{i}^{0 \ldots k}+w_{0}^{0 \ldots k+1}-w_{0}^{0 \ldots k}=w_{i}^{0 \ldots k+1}$. Since $c_{\iota^{\prime}}^{\tau^{\prime}} \subseteq \tau,(7.46)$ implies that

$$
\left(\alpha^{\tau_{1}}\right)^{-1}\left(c_{\iota^{\prime}}^{\tau^{\prime}}\right)=\left(\alpha^{\tau}\right)^{-1}\left(c_{\iota^{\prime}}^{\tau^{\prime}}\right)+w_{0}^{0 \ldots k+1}-w_{0}^{0 \ldots k}
$$

and thus, (7.35) assumes the form

$$
e^{*}=\left(\alpha^{\tau_{1}}\right)^{-1}\left(c_{\iota^{\prime}}^{\tau^{\prime}}\right)+d-w_{0}^{0 \ldots k+1} .
$$

Having $w_{0}^{0 \ldots k+1}$ for its reference point, the right side of (7.45) equals $\left(\alpha^{\tau_{1}}\right)^{-1}\left(c_{\iota_{1}^{\prime}}^{\tau^{\prime}}\right)+d-w_{0}^{0 \ldots k+1}$. Comparing this with (7.48) and taking into account that $d<d_{\iota}^{\tau}$, one concludes that indeed, $e^{*}$ is a face of the right side of (7.45).

Now consider a vertex $w=\left(\alpha^{\tau}\right)^{-1}\left(w_{s}^{0 \ldots k^{\prime}+r}\right) \oplus w_{0}^{0 \ldots k+l}$ of $e^{*}$ and note that $1 \leq l \leq n-k$. Since $\left(\alpha^{\tau}\right)^{-1}\left(c_{l^{\prime}}^{\tau^{\prime}}\right) \oplus d_{\iota}^{\tau}$ and $w_{0}^{0 \ldots k}$ is its reference point, we see that $w=\left(\alpha^{\tau}\right)^{-1}\left(w_{s}^{0 \ldots k^{\prime}+r}\right)+w_{0}^{0 \ldots k+l}-w_{0}^{0 \ldots k}$. Moreover, $w_{s}^{0 \ldots k^{\prime}+r} \in c_{\iota}^{\tau^{\prime}}$ and (7.47) imply that $\left(\alpha^{\tau}\right)^{-1}\left(w_{s}^{0 \ldots k^{\prime}+r}\right)=\left(\alpha^{\tau_{1}}\right)^{-1}\left(w_{s}^{0 \ldots k^{\prime}+r}\right)-w_{0}^{0 \ldots k+1}+w_{0}^{0 \ldots k}$. Consequently, $w=\left(\alpha^{\tau_{1}}\right)^{-1}\left(w_{s}^{0 \ldots \ldots k^{\prime}+r}\right)-w_{0}^{0 \ldots k+1}+w_{0}^{0 \ldots k+l}$. Putting $k_{1}=k+1$, we see that $w=\left(\alpha^{\tau_{1}}\right)^{-1}\left(w_{s}^{0 . \ldots k^{\prime}+r}\right)+w_{0}^{0 \ldots \ldots k_{1}+l-1}-w_{0}^{0 \ldots k_{1}}$. Now (7.33) shows that $\theta_{c_{1}^{*}}(w)=w_{s}^{0 \ldots k^{\prime}} \oplus \frac{1}{2}\left(w_{0}^{0 \ldots k^{\prime}+r}+w_{0}^{0 \ldots k_{1}+l-1}\right)$, the reference point being $w_{0}^{0 \ldots k^{\prime}}$. This coincides with $\theta_{c^{*}}(w)$, given by (7.33), because both direct sums have the same point $w_{0}^{0 \ldots k^{\prime}}$ for their reference point and $k_{1}+l-1=k+l$.

Subcase II.2. Consider the permutation $\pi_{1}$ of $\{k+1, \ldots, n\}$, which interchanges $k+i$ and $k+i+1$ and otherwise coincides with the identity mapping $\eta$ of $\{k+1, \ldots, n\}$. Then the vertices of $d_{\pi_{1}}^{\tau}$ coincide with the vertices of $d_{\eta}^{\tau}$, except for the $i$-th vertex, which equals $w_{0}^{0 \ldots k+i-1 k+i+1}$ and $w_{0}^{0 \ldots k+i}$, respectively. By Lemma 3.8,

$$
d_{\eta}^{\tau} \cap d_{\pi_{1}}^{\tau}=\left[w_{0}^{0 \ldots k}, \ldots, w_{0}^{0 \ldots k+i-1}, w_{0}^{0 \ldots k+i+1}, \ldots, w_{0}^{0 \ldots n}\right]=d
$$


is a common face of $d_{\eta}^{\tau}$ and $d_{\pi_{1}}^{\tau}$. Since the direct sum $\left(\alpha^{\tau}\right)^{-1}\left(c_{\iota^{\prime}}^{\tau^{\prime}}\right) \oplus d_{\pi_{1}}^{\tau}$ is well defined and $w_{0}^{\tau}$ is its reference point, it follows that $e^{*}$, given by $(7.35)$, is a face of that direct sum and thus,

$$
c_{1}^{*}=\left(\alpha^{\tau}\right)^{-1}\left(c_{\iota^{\prime}}^{\tau^{\prime}}\right) \oplus d_{\pi_{1}}^{\tau} .
$$

Now note that the vertex $w=\left(\alpha^{\tau}\right)^{-1}\left(w_{s}^{0 \ldots k^{\prime}+r}\right) \oplus w_{0}^{0 \ldots k+l}$ of $c^{*}$ is a vertex of $e^{*}$ provided $0<l<n-k$ and $l \neq i$. Such a vertex can also be written in the form $w=\left(\alpha^{\tau}\right)^{-1}\left(w_{s}^{0 \ldots k^{\prime}+r}\right) \oplus w_{0}^{0 \ldots k \pi_{1}(k+1) \ldots \pi_{1}(k+l)}$, because $\left\{\pi_{1}(k+1), \ldots, \pi(k+l)\right\}=\{k+1, \ldots, k+l\}$, for $l \neq i$. E.g., for $l=i+1$, one has $\left\{\pi_{1}(k+1), \ldots, \pi_{1}(k+i-1), \pi_{1}(k+i), \pi_{1}(k+i+1)\right\}=\{k+1, \ldots, k+i-1, k+$ $i+1, k+i\}=\{k+1, \ldots, k+i+1\}$. Now (7.27) shows that, for $l \neq i, \theta_{c_{1}^{*}}(w)=$ $w_{s}^{0 \ldots k^{\prime}} \oplus \frac{1}{2}\left(w_{0}^{0 \ldots k^{\prime}+r}+w_{0}^{0 \ldots k \pi_{1}(k+1) \ldots \pi_{1}(k+l)}\right)=w_{s}^{0 \ldots k^{\prime}+r} \oplus \frac{1}{2}\left(w_{0}^{0 \ldots k^{\prime}+r}+w_{0}^{0 \ldots k+l}\right)$, the reference point being $w_{0}^{0 \ldots k^{\prime}}$. However, this expression coincides with the expression (7.33) for $\theta_{c^{*}}(w)$ and thus, $\theta_{c_{1}^{*}}(w)=\theta_{c^{*}}(w)$.

Subcase II.3. This subcase cannot occur. Indeed, $d=\left[w_{0}^{0 \ldots k}, \ldots, w_{0}^{0 \ldots n-1}\right]$ and the vertices $w$ of $e^{*}$ are of the form $w=\left(\alpha^{\tau}\right)^{-1}\left(w_{s}^{0 \ldots k^{\prime}+r}\right) \oplus w_{0}^{0 \ldots k+l}$, where $0 \leq l \leq n-k-1$. Clearly, $\left(\alpha^{\tau}\right)^{-1}\left(w_{s}^{0 \ldots k^{\prime}+r}\right) \subseteq c^{\tau} \subseteq \tau=\left[v_{0}, \ldots, v_{k}\right] \subseteq$ $\left[v_{0}, \ldots, v_{n-1}\right]$, because $k<n$. Furthermore, $w_{0}^{0 \ldots k+l} \in\left[v_{0}, \ldots, v_{k+l}\right] \subseteq$ $\left[v_{0}, \ldots, v_{n-1}\right]$ and $w_{0}^{0 \ldots k} \in \tau \subseteq\left[v_{0}, \ldots, v_{n-1}\right]$. Hence, $w \in\left[v_{0}, \ldots, v_{n-1}\right]$ and thus, also $e^{*} \subseteq\left[v_{0}, \ldots, v_{n-1}\right]$. Since $\left[v_{0}, \ldots, v_{n-1}\right] \subseteq \partial \sigma$, one can have only one $n$-cell, whose face is $e^{*}$.

REmaRK 7.7. Let $\sigma \in K$ and $\operatorname{dim} \sigma=n$. If an $(n-1)$-cell $e^{*}$ from $L^{\prime}(\sigma)$ admits only one $n$-cell $c^{*} \in L^{\prime}(\sigma)$ such that $e^{*}<c^{*}$, then $e^{*}$ is contained in an $(n-1)$-face $\zeta$ of $\sigma$. Indeed, there is no loss of generality in assuming that $\sigma=\left[v_{0}, \ldots, v_{n}\right]$. In the proof of Lemma 7.6, we found a second $n$-cell $c_{1}^{*}$ such that $e^{*}<c_{1}^{*}$ in all but the last Subcase II.3. However, in that situation we proved that $e^{*} \subseteq \zeta=\left[v_{0}, \ldots, v_{n-1}\right]$.

7.6. Step $(v i)$. In Step $(i i)$, for every $n$-simplex $\sigma \in K$, we have defined a function $\vartheta_{n}^{\sigma}: L_{n}^{\prime}(\sigma) \rightarrow N_{n}^{\prime}(\sigma)$ and in Step $(i v)$, for every $n$-cell $c^{*} \in L_{n}^{\prime}(\sigma)$, we have defined an affine isomorphism $\theta_{c^{*}}^{\sigma}: c^{*} \rightarrow c^{\bullet}$, where $c^{\bullet}=\vartheta_{n}^{\sigma}\left(c^{*}\right)$. We will now define functions $\vartheta_{k}^{\sigma}: L_{k}^{\prime}(\sigma) \rightarrow N_{k}^{\prime}(\sigma), 0 \leq k<n$, which together with $\vartheta_{n}^{\sigma}$ form a morphism of cellular complexes $\vartheta^{\sigma}: L^{\prime}(\sigma) \rightarrow N^{\prime}(\sigma)$. Moreover, for every $k$-cell $e^{*} \in L_{k}^{\prime}(\sigma)$, we will define an affine isomorphism $\theta_{e^{*}}^{\sigma}: e^{*} \rightarrow e^{\bullet}$, where $e^{\bullet}=\vartheta_{k}^{\sigma}\left(e^{*}\right)$.

If $e^{*} \in L_{k}^{\prime}(\sigma)$, we choose an $n$-cell $c^{*} \in L^{\prime}(\sigma)$ such that $e^{*}<c^{*}$. Then we put

$$
\vartheta_{k}^{\sigma}\left(e^{*}\right)=\theta_{c^{*}}^{\sigma}\left(e^{*}\right)
$$

Since $\theta_{c^{*}}^{\sigma}$ is an affine isomorphism, $e^{\bullet}=\theta_{c^{*}}^{\sigma}\left(e^{*}\right)$ is a $k$-face of $c^{\bullet}$. We define $\theta_{e^{*}}^{\sigma}$ by putting

$$
\theta_{e^{*}}^{\sigma}=\theta_{c^{*}}^{\sigma} \mid e^{*}
$$


The mapping $\theta_{e^{*}}^{\sigma}$, hence also $e^{\bullet}=\theta_{c^{*}}^{\sigma}\left(e^{*}\right)$, does not depend on the choice of the $n$-cell $c^{*}$. Indeed, if we have another $n$-cell $c_{1}^{*} \in L_{n}^{\prime}(\sigma)$ such that $e^{*}<c_{1}^{*}$, then $e^{*} \subseteq c_{1}^{*} \cap c^{*}$ and thus, by Lemma 7.6, $\theta_{c_{1}^{*}}^{\sigma}\left|e^{*}=\theta_{c^{*}}^{\sigma}\right| e^{*}$. Note that (7.51) and (7.50) imply

$$
\theta_{e^{*}}^{\sigma}\left(e^{*}\right)=\vartheta_{k}^{\sigma}\left(e^{*}\right)
$$

To prove that the functions $\vartheta_{k}^{\sigma}$ preserve faces, consider two cells $e^{*} \in$ $L_{k}^{\prime}(\sigma), e_{1}^{*} \in L_{k_{1}}^{\prime}(\sigma)$ such that $e_{1}^{*}<e^{*}$. Choose an $n$-cell $c^{*} \in L^{\prime}(\sigma)$ such that $e^{*}<c^{*}$, hence also $e_{1}^{*}<c^{*}$. It follows that $\vartheta_{k}^{\sigma}\left(e^{*}\right)=\theta_{c^{*}}^{\sigma}\left(e^{*}\right)$ and $\vartheta_{k_{1}}^{\sigma}\left(e_{1}^{*}\right)=\theta_{c^{*}}^{\sigma}\left(e_{1}^{*}\right)$. Being an affine isomorphism, $\theta_{c^{*}}^{\sigma}: c^{*} \rightarrow c^{\bullet}$ preserves faces. Therefore, $e_{1}^{*}<e^{*}$ implies $\theta_{c^{*}}^{\sigma}\left(e_{1}^{*}\right)<\theta_{c^{*}}^{\sigma}\left(e^{*}\right)$ and thus, $\vartheta_{k_{1}}^{\sigma}\left(e_{1}^{*}\right)<\vartheta_{k}^{\sigma}\left(e^{*}\right)$.

Now consider an $m$-face $\zeta$ of an $n$-simplex $\sigma \in K, 0 \leq m<n$. Since $L^{\prime}(\zeta) \subseteq L^{\prime}(\sigma)$, a $k$-cell $e^{*}$ of $L_{k}^{\prime}(\zeta), 0 \leq k \leq m$, can also be viewed as a $k$-cell of $L^{\prime}(\sigma)$. Let us show that

$$
\theta_{e^{*}}^{\zeta}=\theta_{e^{*}}^{\sigma}
$$

It suffices to consider the case when $\sigma=\left[v_{0}, \ldots, v_{n}\right]$ and $\zeta=\left[v_{0}, \ldots, v_{m}\right]$. Let us prove that $\theta_{e^{*}}^{\zeta}(w)=\theta_{e^{*}}^{\sigma}(w)$, for all the vertices $w$ of $e^{*}$. These vertices are of the form (7.32), where $0 \leq s \leq k^{\prime}, 0 \leq r \leq k-k^{\prime}, 0 \leq l \leq n-k$. By (7.33) for $\zeta$ and $e^{*}$, one sees that $\theta_{e^{*}}^{\zeta}(w)=w_{s}^{0 \ldots k^{\prime}} \oplus \frac{1}{2}\left(w_{0}^{0 \ldots k^{\prime}+r}+w_{0}^{0 \ldots k+l}\right)$. Choose an $n$-cell $c^{*} \in L^{\prime}(\sigma)$ such that $e^{*}<c^{*}$. Viewing $e^{*}$ as an element of $L_{k}^{\prime}(\sigma)$, one concludes that $\theta_{e^{*}}^{\sigma}(w)=\theta_{c^{*}}^{\sigma}(w)$. However, (7.33) for $\sigma$ and $c^{*}$ gives to $\theta_{c^{*}}^{\sigma}(w)$ again the value $w_{s}^{0 \ldots k^{\prime}} \oplus \frac{1}{2}\left(w_{0}^{0 \ldots k^{\prime}+r}+w_{0}^{0 \ldots k+l}\right)$. The fact we just proved implies that the restriction of the function $\vartheta_{k}^{\sigma}: L_{k}^{\prime}(\sigma) \rightarrow N_{k}^{\prime}(\sigma)$ to $L_{k}^{\prime}(\zeta)=L_{k}^{\prime}(\sigma) \cap \zeta$ coincides with $\vartheta_{k}^{\zeta}: L_{k}^{\prime}(\zeta) \rightarrow N_{k}^{\prime}(\zeta)$, i.e.,

$$
\vartheta_{k}^{\zeta}=\vartheta_{k}^{\sigma} \mid L_{k}^{\prime}(\zeta)
$$

Indeed, if $e^{*} \in L_{k}^{\prime}(\zeta),(7.52)$ and (7.53) show that $\vartheta_{k}^{\zeta}\left(e^{*}\right)=\theta_{e^{*}}^{\zeta}\left(e^{*}\right)=$ $\theta_{e^{*}}^{\sigma}\left(e^{*}\right)=\vartheta_{k}^{\sigma}\left(e^{*}\right)$.

LEMMA 7.8. Let $\sigma$ be an n-simplex from $K$. The functions $\vartheta_{k}^{\sigma}, 0 \leq k \leq n$, form an isomorphism of cellular complexes $\vartheta^{\sigma}: L^{\prime}(\sigma) \rightarrow N^{\prime}(\sigma)$.

Proof. We need a morphism of cellular complexes $\varphi^{\sigma}: N^{\prime}(\sigma) \rightarrow L^{\prime}(\sigma)$, consisting of functions $\varphi_{k}^{\sigma}: N_{k}^{\prime}(\sigma) \rightarrow L_{k}^{\prime}(\sigma), 0 \leq k \leq n$, which is inverse to $\vartheta^{\sigma}$. For $k=n$, we put $\varphi_{n}^{\sigma}=\left(\vartheta_{n}^{\sigma}\right)^{-1}: N_{n}^{\prime}(\sigma) \rightarrow L_{n}^{\prime}(\sigma)$. Since $\vartheta_{n}^{\sigma}$ is a bijection, $\varphi_{n}^{\sigma}$ is well defined. If $c^{\bullet} \in N_{n}^{\prime}(\sigma)$ and $c^{*}=\varphi_{n}^{\sigma}\left(c^{\bullet}\right)$, then $c^{\bullet}=\vartheta_{n}^{\sigma}\left(c^{*}\right)$ and the affine isomorphism $\theta_{c^{*}}^{\sigma}: c^{*} \rightarrow c^{\bullet}$ is defined. Therefore, $\phi_{c^{\bullet}}^{\sigma}=\left(\theta_{c^{*}}^{\sigma}\right)^{-1}: c^{\bullet} \rightarrow c^{*}$ is also an affine isomorphism. For $0 \leq k<n$ and $e^{\bullet} \in N_{k}^{\prime}(\sigma)$, we now define $\varphi_{k}^{\sigma}\left(e^{\bullet}\right)$, by choosing an $n$-cell $c^{\bullet} \in N_{n}^{\prime}(\sigma)$ such that $e^{\bullet} \leq c^{\bullet}$ and by putting

$$
\varphi_{k}^{\sigma}\left(e^{\bullet}\right)=\phi_{c}^{\sigma}\left(e^{\bullet}\right)
$$

(formula (7.55) holds also in the case when $k=n$, because then $e^{\bullet}=c^{\bullet}$ ). Clearly, $\phi_{c^{\bullet}}^{\sigma}\left(e^{\bullet}\right)$ is a $k$-face of $\phi_{c^{\bullet}}^{\sigma}\left(c^{\bullet}\right)=c^{*}$. As in the case of $\vartheta^{\sigma}$, to see that 
the functions $\varphi_{k}^{\sigma}, 0 \leq k \leq n$, are well defined and preserve faces, it suffices to prove that

$$
\phi_{c^{\bullet}}^{\sigma}\left|\left(c^{\bullet} \cap c_{1}^{\bullet}\right)=\phi_{c_{1}^{\bullet}}^{\sigma}\right|\left(c^{\bullet} \cap c_{1}^{\bullet}\right),
$$

whenever $c^{\bullet}$ and $c_{1}^{\bullet}$ are $n$-cells of $N^{\prime}(\sigma)$.

As in the proof of Lemma 7.6, it suffices to prove the assertion in the special case, when $c^{\bullet} \cap c_{1}^{\bullet}$ is a common $(n-1)$-face $e^{\bullet}$ of the $n$-cells $c^{\bullet}$ and $c_{1}^{\bullet}$. In that case, $e^{*}=\phi_{c^{\bullet}}^{\sigma}\left(e^{\bullet}\right)$ is an $(n-1)$-face of $c^{*}=\varphi_{n}^{\sigma}\left(c^{\bullet}\right)$. We distinguish two cases: Case $(i)$, when $c^{*}$ is the only $n$-cell from $L^{\prime}(\sigma)$ such that $e^{*}<c^{*}$ and Case $(i i)$, when there is another $n$-cell $c_{1}^{*}$ from $L^{\prime}(\sigma)$ such that $e^{*}<c_{1}^{*}$. In Case $(i)$, Remark 7.7 shows that $e^{*}$ is contained in an $(n-1)$-face $\zeta$ of $\sigma$ and thus, $e^{*} \in L_{n-1}^{\prime}(\sigma) \cap \zeta=L_{n-1}^{\prime}(\zeta)$. Now (7.50) and (7.52) imply that $e^{\bullet}=\theta_{c^{*}}^{\sigma}\left(e^{*}\right)=\vartheta_{n-1}^{\sigma}\left(e^{*}\right)=\vartheta_{n-1}^{\zeta}\left(e^{*}\right) \in N_{n-1}^{\prime}(\zeta)$ and thus, $e^{\bullet} \subseteq \zeta \subseteq \partial \sigma$. However, this is impossible, because $e^{\bullet}$ is the face of two $n$-cells $c^{\bullet}$ and $c_{1}^{\bullet}$. This shows that only Case $(i i)$ is possible.

In Case $(i i), c^{*} \neq c_{1}^{*}$ implies $\vartheta_{n}^{\sigma}\left(c^{*}\right) \neq \vartheta_{n}^{\sigma}\left(c_{1}^{*}\right)$, because $\vartheta_{n}^{\sigma}$ is a bijection. Since $\theta_{c^{*}}^{\sigma}\left(c^{*}\right)=\vartheta_{n}^{\sigma}\left(c^{*}\right)$ and $\theta_{c_{1}^{*}}^{\sigma}\left(c_{1}^{*}\right)=\vartheta_{n}^{\sigma}\left(c_{1}^{*}\right)$, we conclude that $\theta_{c_{1}^{*}}^{\sigma}\left(c_{1}^{*}\right)$ is an $n$-cell from $N^{\prime}(\sigma)$ such that $\theta_{c_{1}^{*}}^{\sigma}\left(c_{1}^{*}\right) \neq \theta_{c^{*}}^{\sigma}\left(c^{*}\right)=c^{\bullet}$. Moreover, $c^{*} \cap c_{1}^{*}=e^{*}$ and Lemma 7.6 show that $\theta_{c_{1}^{*}}^{\sigma}\left|e^{*}=\theta_{c^{*}}^{\sigma}\right| e^{*}$ and thus, $\theta_{c_{1}^{*}}^{\sigma}\left(e^{*}\right)=\theta_{c^{*}}^{\sigma}\left(e^{*}\right)=e^{\bullet}$. Since $e^{*}<c_{1}^{*}$ implies $\theta_{c_{1}^{*}}^{\sigma}\left(e^{*}\right)<\theta_{c_{1}^{*}}^{\sigma}\left(c_{1}^{*}\right)$, we conclude that $\theta_{c_{1}^{*}}^{\sigma}\left(c_{1}^{*}\right)$ is an $n$-cell from $N^{\prime}(\sigma)$, different from $c^{\bullet}$ and such that the $(n-1)$-cell $e^{\bullet}$ is a face of $\theta_{c_{1}^{*}}^{\sigma}\left(c_{1}^{*}\right)$ and $c^{\bullet}$. Since $c^{\bullet}$ and $c_{1}^{\bullet}$ are the only two such $n$-cells, it follows that $\theta_{c_{1}^{*}}^{\sigma}\left(c_{1}^{*}\right)=c_{1}^{\bullet}$ and thus, $\varphi_{n}^{\sigma}\left(c_{1}^{\bullet}\right)=c_{1}^{*}$. Moreover, $\phi_{c_{1}^{\bullet}}^{\sigma}\left|e^{\bullet}=\left(\theta_{c_{1}^{*}}^{\sigma}\right)^{-1}\right| e^{\bullet}$. Since $\theta_{c_{1}^{*}}^{\sigma}\left|e^{*}=\theta_{c^{*}}^{\sigma}\right| e^{*}$, it follows that also $\left(\theta_{c_{1}^{*}}^{\sigma}\right)^{-1}\left|e^{\bullet}=\left(\theta_{c^{*}}^{\sigma}\right)^{-1}\right| e^{\bullet}$ and thus, $\phi_{c_{1}^{\bullet}}^{\sigma}\left|e^{\bullet}=\phi_{c^{\bullet}}^{\sigma}\right| e^{\bullet}$.

Let us now show that the functions $\varphi_{k}^{\sigma}: N_{k}^{\prime}(\sigma) \rightarrow L_{k}^{\prime}(\sigma), 0 \leq k \leq n$, preserve faces and therefore, they form a morphism of cellular complexes $\varphi^{\sigma}: N^{\prime}(\sigma) \rightarrow L^{\prime}(\sigma)$. Consider two cells $e^{\bullet} \in N_{k}^{\prime}(\sigma)$ and $e_{1}^{\bullet} \in N_{k_{1}}^{\prime}(\sigma)$ such that $e_{1}^{\bullet}<e^{\bullet}$. Choose an $n$-cell $c^{\bullet} \in N_{n}^{\prime}(\sigma)$ such that $e^{\bullet}<c^{\bullet}$, hence also $e_{1}^{\bullet}<c^{\bullet}$. It follows, by (7.55), that $\varphi_{k}^{\sigma}\left(e^{\bullet}\right)=\phi_{c}^{\sigma}\left(e^{\bullet}\right)$ and $\varphi_{k_{1}}^{\sigma}\left(e_{1}^{\bullet}\right)=\phi_{c}^{\sigma}\left(e_{1}^{\bullet}\right)$. Being an affine isomorphism, $\phi_{c^{\bullet}}^{\sigma}: c^{\bullet} \rightarrow c^{*}=\varphi_{n}^{\sigma}\left(c^{\bullet}\right)$ preserves faces. Therefore, $e_{1}^{\bullet}<e^{\bullet}$ implies $\phi_{c^{\bullet}}^{\sigma}\left(e_{1}^{\bullet}\right)<\phi_{c^{\bullet}}^{\sigma}\left(e^{\bullet}\right)$ and thus, $\varphi_{k_{1}}^{\sigma}\left(e_{1}^{\bullet}\right)<\varphi_{k}^{\sigma}\left(e^{\bullet}\right)$.

To complete the proof of Lemma 7.8, it suffices to show that, for $0 \leq k \leq$ $n$, the function $\varphi_{k}^{\sigma}: N_{k}^{\prime}(\sigma) \rightarrow L_{k}^{\prime}(\sigma)$ is the inverse of the function $\vartheta_{k}^{\sigma}: L_{k}^{\prime}(\sigma) \rightarrow$ $N_{k}^{\prime}(\sigma)$, i.e.,

$$
\varphi_{k}^{\sigma} \vartheta_{k}^{\sigma}=\mathrm{id}, \vartheta_{k}^{\sigma} \varphi_{k}^{\sigma}=\mathrm{id} .
$$

Indeed, $\varphi_{n}^{\sigma} \vartheta_{n}^{\sigma}\left(c^{*}\right)=c^{*}$, for $c^{*} \in L_{n}^{\prime}(\sigma)$. If $e^{*}$ is a $k$-cell of $L^{\prime}(\sigma), 0 \leq k<n$, and $c^{*}$ is an $n$-cell of $L^{\prime}(\sigma)$ such that $e^{*}<c^{*}$, then (7.50) shows that $\bar{\vartheta}_{k}^{\sigma}\left(e^{*}\right)=$ $\theta_{c^{*}}^{\sigma}\left(e^{*}\right)$. Clearly, $e^{\bullet}=\theta_{c^{*}}^{\sigma}\left(e^{*}\right)<\theta_{c^{*}}^{\sigma}\left(c^{*}\right)=c^{\bullet}$. Consequently, $\varphi_{k}^{\sigma} \vartheta_{k}^{\sigma}\left(e^{*}\right)=$ $\varphi_{k}^{\sigma} \theta_{c^{*}}^{\sigma}\left(e^{*}\right)=\varphi_{k}^{\sigma}\left(e^{\bullet}\right)=\phi_{c^{\bullet}}^{\sigma}\left(e^{\bullet}\right)=\left(\theta_{c^{*}}^{\sigma}\right)^{-1}\left(\theta_{c^{*}}^{\sigma}\left(e^{*}\right)\right)=e^{*}$. Similarly, $\vartheta_{n}^{\sigma} \varphi_{n}^{\sigma}\left(c^{\bullet}\right)=$ $c^{\bullet}$, for $c^{\bullet} \in N_{n}^{\prime}(\sigma)$. If $e^{\bullet}$ is a $k$-cell of $N^{\prime}(\sigma), 0 \leq k<n$, and $c^{\bullet}$ is an $n$ cell of $N^{\prime}(\sigma)$ such that $e^{\bullet}<c^{\bullet}$, then (7.55) shows that $\varphi_{k}^{\sigma}\left(e^{\bullet}\right)=\phi_{c}^{\sigma} \bullet\left(e^{\bullet}\right)$. 
Clearly, $e^{*}=\phi_{c}^{\sigma} \bullet\left(e^{\bullet}\right)<\phi_{c}^{\sigma} \cdot\left(c^{\bullet}\right)=c^{*}$. Consequently, $\vartheta_{k}^{\sigma} \varphi_{k}^{\sigma}\left(e^{\bullet}\right)=\vartheta_{k}^{\sigma} \phi_{c}^{\sigma}\left(e^{\bullet}\right)=$ $\vartheta_{k}^{\sigma}\left(e^{*}\right)=\theta_{c^{*}}^{\sigma}\left(e^{*}\right)=\theta_{c^{*}}^{\sigma}\left(\left(\theta_{c^{*}}^{\sigma}\right)^{-1}\left(e^{\bullet}\right)\right)=e^{\bullet}$. This completes the proof that $\varphi^{\sigma}$ is an inverse of $\vartheta^{\sigma}$ and thus, $\vartheta^{\sigma}$ is an isomorphism of cellular complexes.

7.7. Step (vii). For every $n$-simplex $\sigma \in K$, we have defined in Step (vi) functions $\vartheta_{k}^{\sigma}: L_{k}^{\prime}(\sigma) \rightarrow N_{k}^{\prime}(\sigma), 0 \leq k \leq n$, which form an isomorphism of cellular complexes $\vartheta^{\sigma}: L^{\prime}(\sigma) \rightarrow N^{\prime}(\sigma)$. Moreover, for every $k$-cell $e^{*} \in L_{k}^{\prime}(\sigma)$, we have defined an affine isomorphism $\theta_{e^{*}}^{\sigma}: e^{*} \rightarrow e^{\bullet}$, where $e^{\bullet}=\vartheta_{k}^{\sigma}\left(e^{*}\right)$. We will now define a sequence of face-preserving functions $\vartheta_{k}: L_{k}^{\prime}(K) \rightarrow N_{k}^{\prime}(K)$, $k \in\{0,1, \ldots\}$, which form an isomorphism of cellular complexes $\vartheta: L^{\prime}(K) \rightarrow$ $N^{\prime}(K)$. We put

If $e^{*} \in L_{k}^{\prime}(K)$, then there exists a simplex $\sigma \in K$ such that $e^{*} \in L_{k}^{\prime}(\sigma)$.

$$
\vartheta_{k}\left(e^{*}\right)=\vartheta_{k}^{\sigma}\left(e^{*}\right) .
$$

Note that $\vartheta_{k}^{\sigma}\left(e^{*}\right) \in N_{k}^{\prime}(\sigma) \subseteq N_{k}^{\prime}(K)$. Let us prove that $\vartheta_{k}\left(e^{*}\right)$ does not depend on the choice of $\sigma$. Indeed, assume that $\sigma_{1}$ is another simplex of $K$ such that $e^{*} \in L_{k}^{\prime}\left(\sigma_{1}\right)$. Note that $\sigma \cap \sigma_{1} \in K$ and $\sigma \cap \sigma_{1} \leq \sigma, \sigma \cap \sigma_{1} \leq \sigma_{1}$. Also note that $e^{*} \subseteq \sigma \cap \sigma_{1}$. Consequently, by Theorem 5.1, $e^{*} \in L_{k}^{\prime}(\sigma) \cap\left(\sigma \cap \sigma_{1}\right)=L_{k}^{\prime}\left(\sigma \cap \sigma_{1}\right)$. For $\zeta=\sigma \cap \sigma_{1} \leq \sigma,(7.54)$ shows that $\vartheta_{k}^{\sigma \cap \sigma_{1}}\left(e^{*}\right)=\vartheta_{k}^{\sigma}\left(e^{*}\right)$. Analogously, $\sigma \cap \sigma_{1} \leq \sigma_{1}$ implies $\vartheta_{k}^{\sigma \cap \sigma_{1}}\left(e^{*}\right)=\vartheta_{k}^{\sigma_{1}}\left(e^{*}\right)$ and thus, $\vartheta_{k}^{\sigma}\left(e^{*}\right)=\vartheta_{k}^{\sigma_{1}}\left(e^{*}\right)$.

To prove that the functions $\vartheta_{k}$ preserve faces, consider two cells $e^{*} \in$ $L_{k}^{\prime}(K), e_{1}^{*} \in L_{k_{1}}^{\prime}(K)$ such that $e_{1}^{*}<e^{*}$. Choose a simplex $\sigma \in K$ such that $e^{*} \in L_{k}^{\prime}(\sigma)$. Then $\vartheta_{k}\left(e^{*}\right)=\vartheta_{k}^{\sigma}\left(e^{*}\right)$. Since $L_{k}^{\prime}(\sigma)$ is a cellular complex, $e^{*} \in L_{k}^{\prime}(\sigma)$ and $e_{1}^{*}<e^{*}$ imply that also $e_{1}^{*} \in L_{k}^{\prime}(\sigma)$. Consequently, $\vartheta_{k_{1}}\left(e_{1}^{*}\right)=\vartheta_{k_{1}}^{\sigma}\left(e_{1}^{*}\right)$. Taking into account that the functions $\vartheta_{k}^{\sigma}$, $0 \leq k \leq \operatorname{dim} \sigma$, preserve faces, one concludes that $\vartheta_{k_{1}}^{\sigma}\left(e_{1}^{*}\right)<\vartheta_{k}^{\sigma}\left(e^{*}\right)$ and thus, $\vartheta_{k}\left(e_{1}^{*}\right)<\vartheta_{k}\left(e^{*}\right)$. Consequently, the functions $\vartheta_{k}: L_{k}^{\prime}(K) \rightarrow N_{k}^{\prime}(K)$, $k \in\{0,1, \ldots\}$, form a morphism of cellular complexes $\vartheta: L^{\prime}(K) \rightarrow N^{\prime}(K)$. By (7.58), $\vartheta_{k}\left|L_{k}^{\prime}(\sigma)=\vartheta_{k}^{\sigma}\right| L_{k}^{\prime}(\sigma), 0 \leq k \leq n, \operatorname{dim} \sigma=n$. Therefore, Lemma 7.8 shows that the restrictions $\vartheta_{k} \mid L_{k}^{\prime}(\sigma), 0 \leq k \leq n$, form an isomorphism of cellular complexes $\vartheta^{\sigma}: L_{k}^{\prime}(\sigma) \rightarrow N_{k}^{\prime}(\sigma)$.

The proof of Theorem 7.1 will be completed if we prove the following lemma.

Lemma 7.9. The functions $\vartheta_{k}: L_{k}^{\prime}(K) \rightarrow N_{k}^{\prime}(K), k \in\{0,1, \ldots\}$, form an isomorphism of cellular complexes $\vartheta: L^{\prime}(K) \rightarrow N^{\prime}(K)$.

Proof. To prove the lemma we need a sequence of functions $\varphi_{k}: N_{k}^{\prime}(K)$ $\rightarrow L_{k}^{\prime}(K), k \in\{0,1, \ldots\}$, which forms an inverse $\varphi: N^{\prime}(K) \rightarrow L^{\prime}(K)$ of the morphism of cellular complexes $\vartheta: L^{\prime}(K) \rightarrow N^{\prime}(K)$. If $e^{\bullet} \in N_{k}^{\prime}(K)$, then there exists a simplex $\sigma \in K$ such that $e^{\bullet} \in N_{k}^{\prime}(\sigma)$. We put

$$
\varphi_{k}\left(e^{\bullet}\right)=\varphi_{k}^{\sigma}\left(e^{\bullet}\right),
$$


where $\varphi_{k}^{\sigma}$ is the inverse of $\vartheta_{k}^{\sigma}$. Note that $\varphi_{k}^{\sigma}\left(e^{\bullet}\right) \in L_{k}^{\prime}(\sigma) \subseteq L_{k}^{\prime}(K)$. We will prove that $\varphi_{k}\left(e^{\bullet}\right)$ does not depend on the choice of $\sigma$ and therefore, $\varphi_{k}: N_{k}^{\prime}(K) \rightarrow L_{k}^{\prime}(K)$ is well defined.

Let us first prove the analogue of (7.54), i.e., let us prove that $\zeta<\sigma$ implies

$$
\varphi_{k}^{\zeta}=\varphi_{k}^{\sigma} \mid N_{k}^{\prime}(\zeta)
$$

For $e^{\bullet} \in N_{k}^{\prime}(\zeta)$, put $e^{*}=\varphi_{k}^{\zeta}\left(e^{\bullet}\right) \in L_{k}^{\prime}(\zeta)$. Then $\vartheta_{k}^{\zeta}\left(e^{*}\right)=\vartheta_{k}^{\zeta} \varphi_{k}^{\zeta}\left(e^{\bullet}\right)=e^{\bullet}$. By (7.54), $\vartheta_{k}^{\zeta}\left(e^{*}\right)=\vartheta_{k}^{\sigma}\left(e^{*}\right)$ and thus, $\vartheta_{k}^{\sigma}\left(e^{*}\right)=e^{\bullet}$. Consequently, $e^{*}=$ $\varphi_{k}^{\sigma} \vartheta_{k}^{\sigma}\left(e^{*}\right)=\varphi_{k}^{\sigma}\left(e^{\bullet}\right)$. Comparing this with $e^{*}=\varphi_{k}^{\zeta}\left(e^{\bullet}\right)$, one concludes that indeed, $\varphi_{k}^{\sigma}\left(e^{\bullet}\right)=\varphi_{k}^{\zeta}\left(e^{\bullet}\right)$.

Now assume that $\sigma, \sigma_{1} \in K$ and $e^{\bullet} \in N_{k}^{\prime}(\sigma) \cap N_{k}^{\prime}\left(\sigma_{1}\right)$. We have to prove that $\varphi_{k}^{\sigma}\left(e^{\bullet}\right)=\varphi_{k}^{\sigma_{1}}\left(e^{\bullet}\right)$. Since $\left|N_{k}^{\prime}(\sigma)\right| \subseteq \sigma$ and $\left|N_{k}^{\prime}\left(\sigma_{1}\right)\right| \subseteq \sigma_{1}$, it follows that $e^{\bullet} \subseteq \sigma \cap \sigma_{1}$. Since $\zeta=\sigma \cap \sigma_{1} \in K$, one concludes that $e^{\bullet} \in N_{k}^{\prime}(\sigma) \cap \zeta=N_{k}^{\prime}(\zeta)$. Since $\zeta \leq \sigma,(7.60)$ implies that $\varphi_{k}^{\sigma}\left(e^{\bullet}\right)=\varphi_{k}^{\zeta}\left(e^{\bullet}\right)=\varphi_{k}^{\sigma_{1}}\left(e^{\bullet}\right)$.

To prove that the functions $\varphi_{k}$ preserve faces, consider two cells $e^{\bullet} \in$ $N^{\prime}(K), e_{1}^{\bullet} \in N^{\prime}(K)$, such that $e_{1}^{*}<e^{*}$. Choose an $n$-simplex $\sigma \in K$ such that $e^{\bullet} \in N_{k}^{\prime}(\sigma)$. Then $\varphi_{k}\left(e^{\bullet}\right)=\varphi_{k}^{\sigma}\left(e^{\bullet}\right)$. Since $N_{k}^{\prime}(\sigma)$ is a cellular complex, $e^{\bullet} \in N_{k}^{\prime}(\sigma)$ and $e_{1}^{\bullet}<e^{\bullet}$ imply that also $e_{1}^{\bullet} \in N_{k}^{\prime}(\sigma)$. Consequently, $\varphi_{k_{1}}\left(e_{1}^{\bullet}\right)=\varphi_{k_{1}}^{\sigma}\left(e_{1}^{\bullet}\right)$. Taking into account that the functions $\varphi_{k}^{\sigma}, 0 \leq k \leq n$, preserve faces, one concludes that $\varphi_{k_{1}}^{\sigma}\left(e_{1}^{\bullet}\right)<\varphi_{k}^{\sigma}\left(e^{\bullet}\right)$ and thus, $\varphi_{k}\left(e_{1}^{\bullet}\right)<\varphi_{k}\left(e^{\bullet}\right)$. Consequently, the functions $\varphi_{k}: N_{k}^{\prime}(K) \rightarrow L_{k}^{\prime}(K), k \in\{0,1, \ldots\}$, form a morphism of cellular complexes $\varphi: N^{\prime}(K) \rightarrow L^{\prime}(K)$.

To complete the proof of Lemma 7.9 , it suffices to show that the morphism $\varphi$ is an inverse of $\vartheta$, i.e., for $k \in\{0,1, \ldots\}$,

$$
\varphi_{k} \vartheta_{k}=\mathrm{id}, \vartheta_{k} \varphi_{k}=\mathrm{id} \text {. }
$$

If $e^{*} \in L_{k}^{\prime}(K)$, choose a simplex $\sigma \in K$ such that $e^{*} \in L_{k}^{\prime}(\sigma)$. By (7.58), $\vartheta_{k}\left(e^{*}\right)=\vartheta_{k}^{\sigma}\left(e^{*}\right) \in N_{k}^{\prime}(\sigma)$ and by (7.59) and (7.57), $\varphi_{k} \vartheta_{k}\left(e^{*}\right)=\varphi_{k}^{\sigma} \vartheta_{k}^{\sigma}\left(e^{*}\right)=$ $e^{*}$. Analogously, if $e^{\bullet} \in N_{k}^{\prime}(K)$, choose a simplex $\sigma \in K$ such that $e^{\bullet} \in N_{k}^{\prime}(\sigma)$. By $(7.59), \varphi_{k}\left(e^{\bullet}\right)=\varphi_{k}^{\sigma}\left(e^{\bullet}\right) \in L_{k}^{\prime}(\sigma)$ and by (7.58) and (7.57), $\vartheta_{k} \varphi_{k}\left(e^{\bullet}\right)=$ $\vartheta_{k}^{\sigma} \varphi_{k}^{\sigma}\left(e^{\bullet}\right)=e^{\bullet}$.

7.8. Step (viii). In Step $(v)$, for an $n$-simplex $\sigma \in K$, we have defined a mapping $\theta^{\sigma}: \sigma \rightarrow \sigma$, by putting $\theta^{\sigma} \mid c^{*}=\theta_{c^{*}}^{\sigma}$, for every $n$-cell $c^{*} \in L_{n}^{\prime}(\sigma)$.

Lemma 7.10. For every $n$-simplex $\sigma \in K$, the mapping $\theta^{\sigma}: \sigma \rightarrow \sigma$ is a selfhomeomorphism. For every $k$-cell $c^{*} \in L_{k}^{\prime}(\sigma), 0 \leq k \leq n$, the restriction $\theta^{\sigma} \mid c^{*}$ is an affine isomorphism $c^{*} \rightarrow \vartheta_{k}\left(c^{*}\right)$.

Proof. We will define a mapping $\phi^{\sigma}: \sigma \rightarrow \sigma$, which is the inverse of $\theta^{\sigma}$, by putting

$$
\phi^{\sigma} \mid c^{\bullet}=\phi_{c}^{\sigma}
$$


where $c^{\bullet} \in N_{n}^{\prime}(\sigma)$. This mapping is well defined, because of (7.56). One has to show that

$$
\phi^{\sigma} \theta^{\sigma}=\mathrm{id}, \theta^{\sigma} \phi^{\sigma}=\mathrm{id} .
$$

Since $\phi_{c^{\bullet}}^{\sigma}=\left(\theta_{c^{*}}^{\sigma}\right)^{-1}$, where $c^{*}=\vartheta_{n}^{\sigma}\left(c^{\bullet}\right)$, we see that $\theta^{\sigma} \phi^{\sigma} \mid c^{\bullet}=\theta^{\sigma} \phi_{c^{\bullet}}^{\sigma}=$ $\theta_{c^{*}}^{\sigma} \phi_{c^{\bullet}}^{\sigma}=$ id. Since the $n$-cells $c^{\bullet} \in N_{n}^{\prime}(\sigma)$ cover $\sigma$, we conclude that $\theta^{\sigma} \phi^{\sigma}=$ id. Analogously, $\phi^{\sigma} \theta^{\sigma}=\mathrm{id}$.

Step (ix). We now define a mapping $\theta: P \rightarrow P, P=|K|$, by putting

$$
\theta \mid \sigma=\theta^{\sigma},
$$

for $\sigma \in K$. To see that this mapping is well defined, it suffices to show that, for any two simplices $\sigma, \sigma_{1} \in K$, one has $\theta^{\sigma}\left|\left(\sigma \cap \sigma_{1}\right)=\theta^{\sigma_{1}}\right|\left(\sigma \cap \sigma_{1}\right)$. Let us first show that

$$
\theta^{\sigma} \mid \zeta=\theta^{\zeta}
$$

for $\zeta<\sigma$. Indeed, if $\operatorname{dim} \zeta=m$, then $\zeta=\left|L_{m}^{\prime}(\zeta)\right|$. Therefore, it suffices to show that $\theta^{\sigma}\left|e^{*}=\theta^{\zeta}\right| e^{*}$, for every $m$-cell $e^{*} \in L_{m}^{\prime}(\zeta)$. Since $e^{*} \in \zeta \subseteq \sigma,(7.28)$ shows that $\theta^{\zeta} \mid e^{*}=\theta_{e^{*}}^{\zeta}$ and $\theta^{\sigma} \mid e^{*}=\theta_{e^{*}}^{\sigma}$. However, (7.53) shows that $\theta_{e^{*}}^{\zeta}=\theta_{e^{*}}^{\sigma}$ and thus, $\theta^{\sigma}\left|e^{*}=\theta^{\zeta}\right| e^{*}$. Now put $\zeta=\sigma \cap \sigma_{1}$ and note that $\zeta \leq \sigma$ and $\zeta \leq \sigma_{1}$. Consequently, (7.64) implies that $\theta^{\sigma}\left|\zeta=\theta^{\zeta}=\theta^{\sigma_{1}}\right| \zeta$, as desired. Note that (7.63) and Lemma 7.10 imply that the mapping $\theta: P \rightarrow P$ has the following property. For every $\sigma \in K$ and every $k$-cell $c^{*} \in L^{\prime}(\sigma)$, the restriction $\theta \mid c^{*}$ is an affine isomorphism between $c^{*}$ and the $k$-cell $\vartheta_{k}\left(c^{*}\right) \in N^{\prime}(K)$.

7.9. In view of Lemma 7.10, the following lemma will complete the proof of Theorem 7.2.

Lemma 7.11. The mapping $\theta: P \rightarrow P$ is a selfhomeomorphism of $P$. Moreover, for every simplex $\sigma \in K$, the restriction $\theta \mid \sigma$ is a selfhomeomorphism of $\sigma$.

Proof. We define a mapping $\phi: P \rightarrow P$, which is the inverse of $\theta$, by putting

$$
\phi \mid \sigma=\phi^{\sigma},
$$

for $\sigma \in K$. To see that this mapping is well defined, it suffices to show that, for any two simplices $\sigma, \sigma_{1} \in K$, one has $\phi^{\sigma}\left|\left(\sigma \cap \sigma_{1}\right)=\phi^{\sigma_{1}}\right|\left(\sigma \cap \sigma_{1}\right)$. An argument already used in Step $(i x)$ shows that it suffices to prove that $\phi^{\sigma} \mid \zeta=\phi^{\zeta}$, for $\zeta<\sigma$. If $\operatorname{dim} \zeta=m$, then the $m$-cells $e^{\bullet} \in N_{m}^{\prime}(\zeta)$ cover $\zeta$. Therefore, it suffices to show that $\phi^{\sigma}\left|e^{\bullet}=\phi^{\zeta}\right| e^{\bullet}$, for every $m$-cell $e^{\bullet} \in N_{m}^{\prime}(\zeta)$. Since $N_{m}^{\prime}(\zeta) \subseteq N_{m}^{\prime}(\sigma),(7.62)$ shows that $\phi^{\zeta} \mid e^{\bullet}=\phi_{e^{\bullet}}^{\zeta}$ and $\phi^{\sigma} \mid e^{\bullet}=\phi_{e^{\bullet}}^{\sigma}$. However, by (7.53), one has $\theta_{e^{*}}^{\zeta}=\theta_{e^{*}}^{\sigma}$. Since $\phi_{e^{\bullet}}^{\zeta}=\left(\theta_{e^{*}}^{\zeta}\right)^{-1}$ and $\phi_{e^{\bullet}}^{\sigma}=\left(\theta_{e^{*}}^{\sigma}\right)^{-1}$, one concludes that $\phi_{e^{\zeta}}^{\zeta}=\phi_{e^{\bullet}}^{\sigma}$ and thus, $\phi^{\sigma}\left|e^{\bullet}=\phi^{\zeta}\right| e^{\bullet}$.

To complete the proof that $\theta$ is a selfhomeomorphism, we now only have to prove that $\phi \theta=$ id and $\theta \phi=$ id. To prove the first of these relations, it 
Suffices to prove that $(\phi \theta) \mid \sigma=\mathrm{id}$, because the simplices $\sigma \in K$ cover $P=|K|$. However, by (7.64) and (7.66), one has $(\phi \theta) \mid \sigma=\phi \theta^{\sigma}=\phi^{\sigma} \theta^{\sigma}$ and by (7.63), one has $\phi^{\sigma} \theta^{\sigma}=\mathrm{id}$. Consequently, $\phi \theta=\mathrm{id}$. One proves analogously, that also $\theta \phi=\mathrm{id}$.

To complete the proof of Lemma 7.11, it only remains to see that $\theta \mid \sigma: \sigma \rightarrow$ $\sigma$ is a selfhomeomorphism, for every simplex $\sigma \in K$. Indeed, (7.64) shows that $\theta \mid \sigma=\theta^{\sigma}$ and Lemma 7.10 asserts that $\theta^{\sigma}$ is a selfhomeomorphism of $\sigma$.

\title{
ACKNOWLEDGEMENTS.
}

The author is grateful to the referee for his meticulous reading of the paper. He detected an error in the proof of Theorem 6.1, which has now been corrected. He also pointed out a number of minor errors and misprints as well as many places, where the presentation in the original manuscript needed improvement.

\section{REFERENCES}

[1] P. Alexandroff and H. Hopf, Topologie, Springer, Berlin, 1935.

[2] A. Brøndsted, An introduction to convex polytopes, Graduate Texts in Mathematics, Springer, Berlin, 1983.

[3] J. Dugundji, Topology, Allyn and Bacon, Boston, 1966.

[4] B. Grünbaum, Convex polytopes, Interscience Publishers John Wiley \& Sons, Inc., New York, 1967.

[5] S. Mardešić, Strong shape and homology, Springer Monographs in Mathematics, Springer-Verlag, Berlin, 2000.

[6] S. Mardešić, A resolution for the product of a compactum with a polyhedron, Topology Appl. 133 (2003), 37-63.

[7] S. Mardešić, Functoriality of the standard resolution of the Cartesian product of a compactum and a polyhedron, Topology Appl. 155 (2007), 1-32.

[8] S. Mardešić, J. Segal, Shape theory, North-Holland, Amsterdam, 1982.

[9] M. Moszyńska, Selected topics in convex geometry, Birkhäuser, Basel, 2006.

\author{
S. Mardešić \\ Department of Mathematics \\ University of Zagreb \\ Bijenička cesta 30, 10002 Zagreb, P.O. Box 335 \\ Croatia \\ E-mail: smardes@math.hr \\ Received: 11.4.2007. \\ Revised: 16.4.2009.
}

